\title{
Synthesis of Amino Terminal Clicked dendrimers. Approaches to the application as a biomarker
}

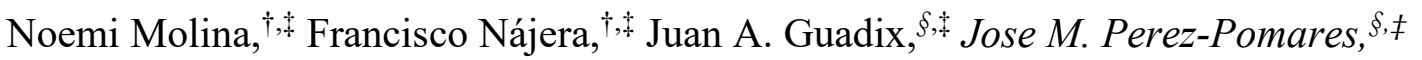 \\ Yolanda Vida, ${ }^{*}, \dagger,+$ and Ezequiel Perez-Inestrosa ${ }^{*} \dagger, *$ \\ † Universidad de Málaga-IBIMA, Departamento de Química Orgánica. Campus de Teatinos s/n, 29071- \\ Málaga, Spain \\ $\$$ Centro Andaluz de Nanomedicina y Biotecnología-BIONAND. Parque Tecnológico de Andalucía, C/ \\ Severo Ochoa 35, 29590, Campanillas, Málaga, Spain \\ $\S$ Universidad de Málaga-IBIMA Departamento de Biología Animal. Campus de Teatinos s/n, 29071- \\ Málaga, Spain
}

\section{Table of contents}

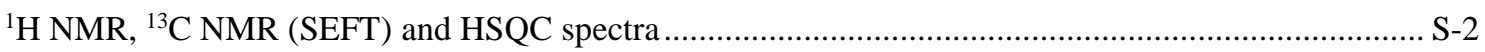

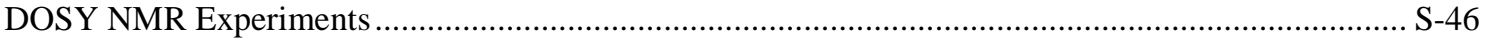

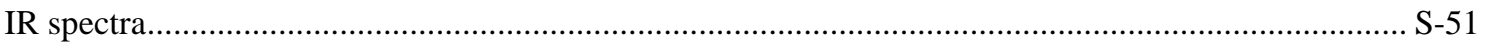

Luminescent Properties of $N$-(2-aminoethyl)-4-((2-aminoethyl)amino)-1,8-naphthalimide ................ S-54

Luminescent Microscopy experiments with E. coli bacteria incubated with $\mathrm{G}_{\mathrm{Naph}_{\mathrm{NH}}}\left(5 \cdot 10^{-4} \mathrm{M}\right) \ldots . . \mathrm{S}-54$

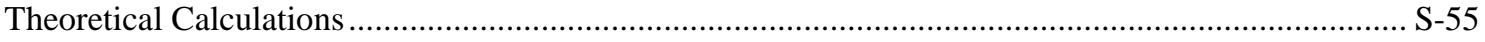

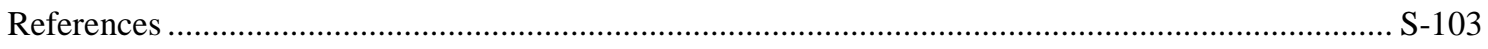


${ }^{1} \mathrm{H}$ NMR, ${ }^{13} \mathrm{C}$ NMR (SEFT) and HSQC spectra

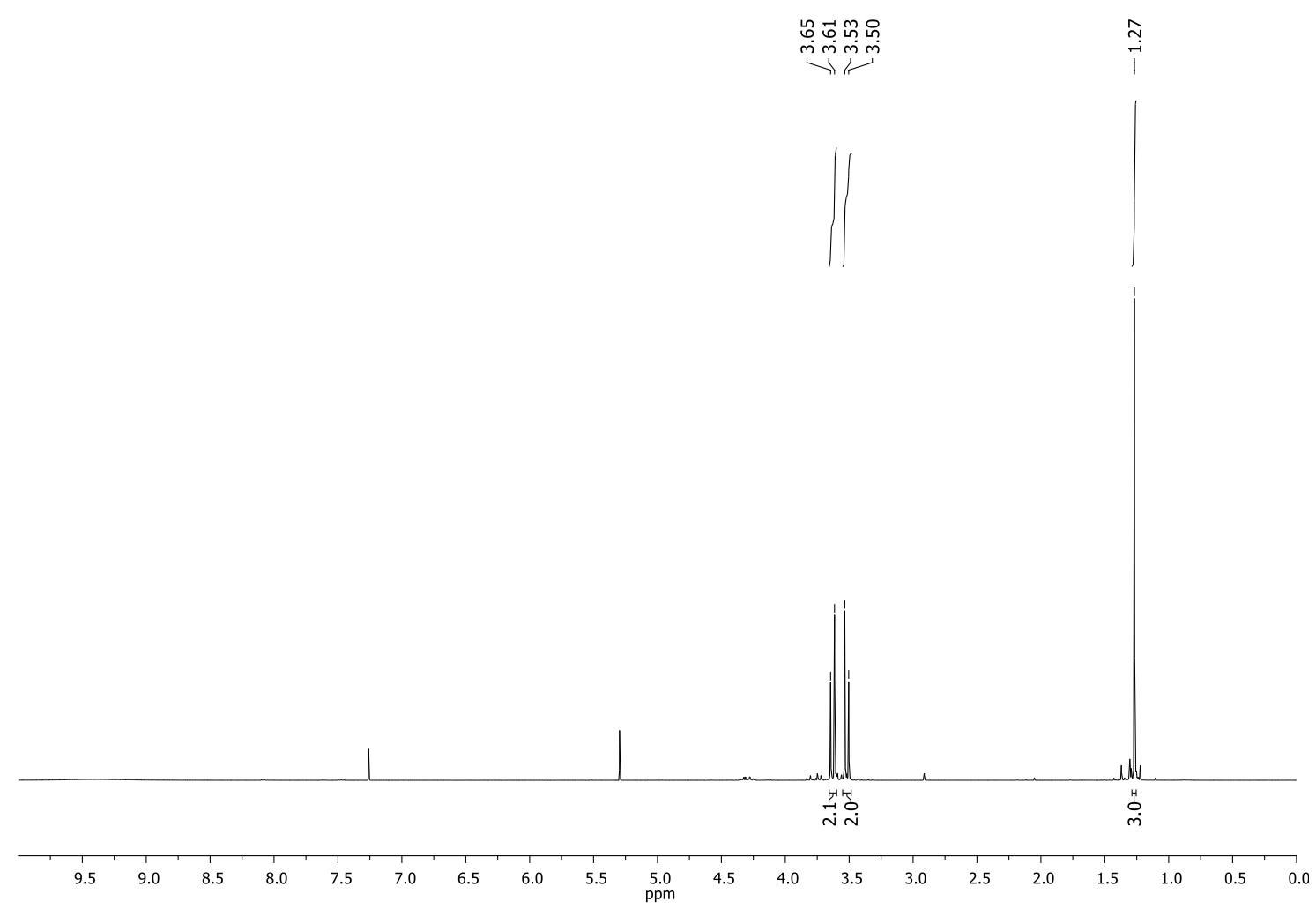

Figure S1. ${ }^{1} \mathrm{H}$ NMR spectrum of 3,3'-diazidopivalic acid in $\mathrm{CDCl}_{3}$.
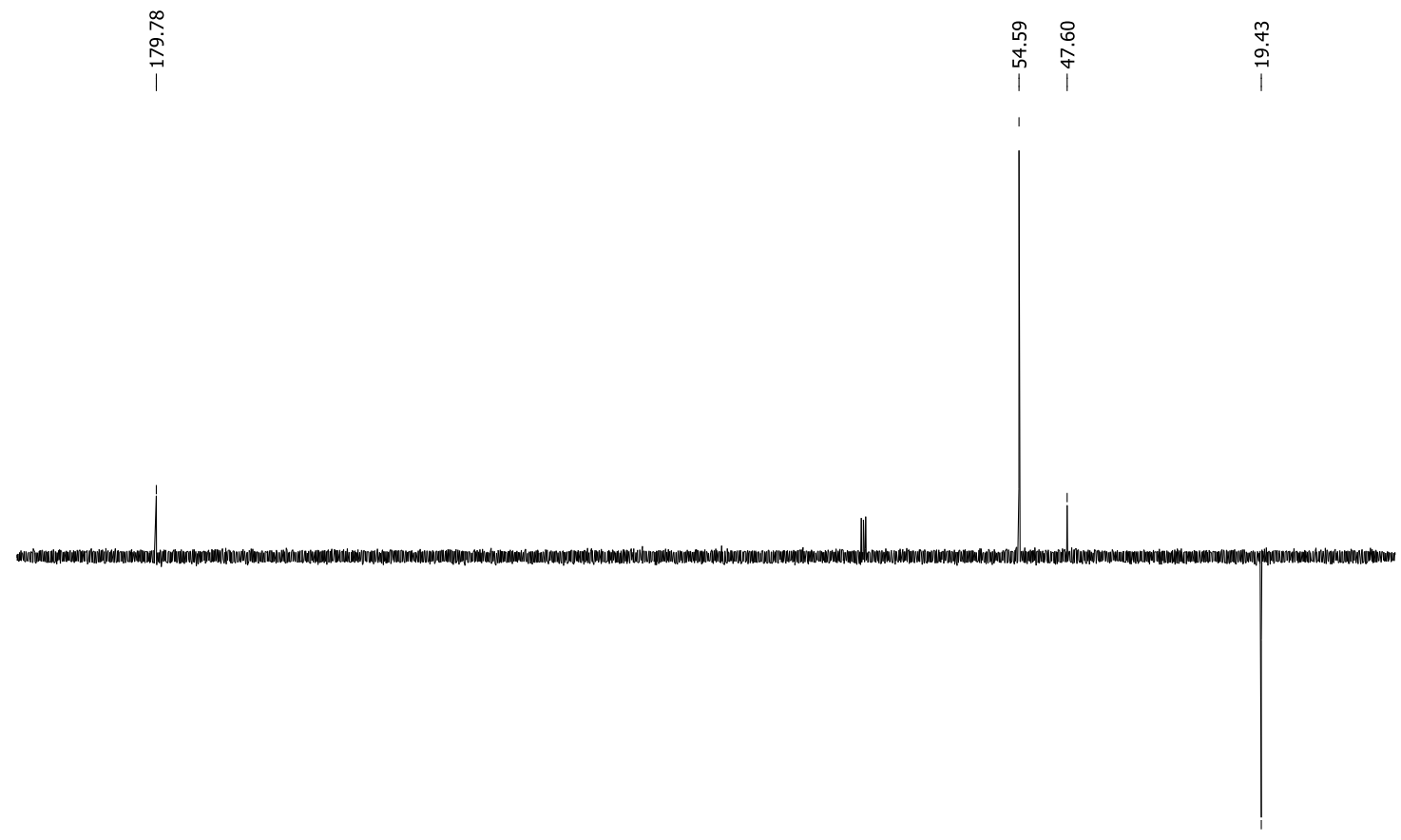

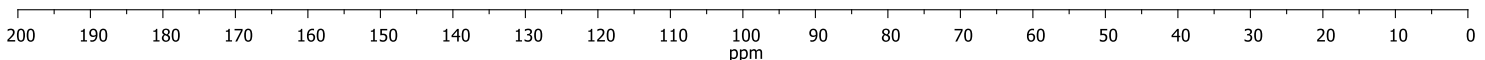

Figure S2. ${ }^{13} \mathrm{C}$ NMR (SEFT) spectrum of 3,3'-diazidopivalic acid in $\mathrm{CDCl}_{3}$. 


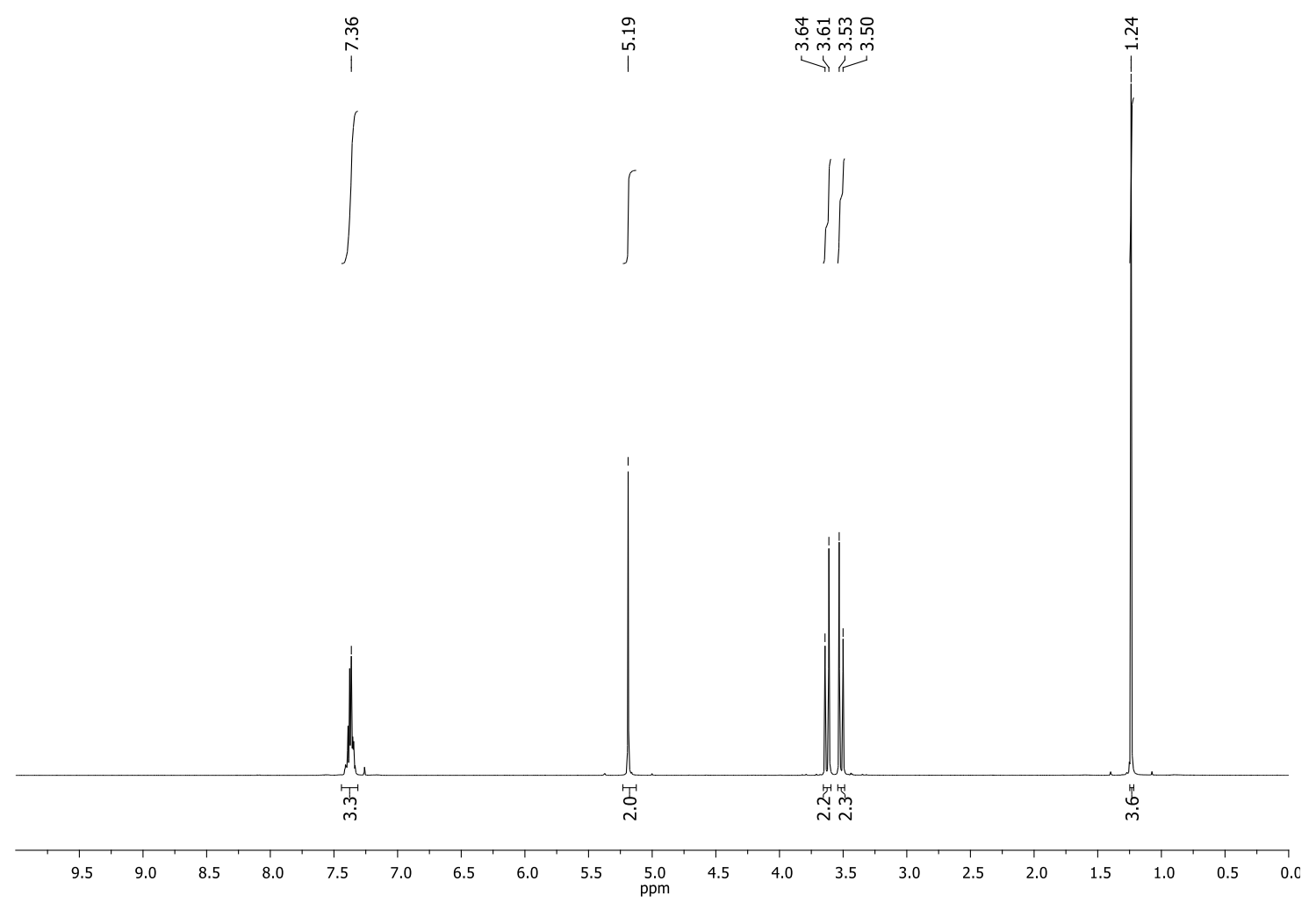

Figure S3. ${ }^{1} \mathrm{H}$ NMR spectrum of benzyl-3,3'-diazidopivaloate in $\mathrm{CDCl}_{3}$.

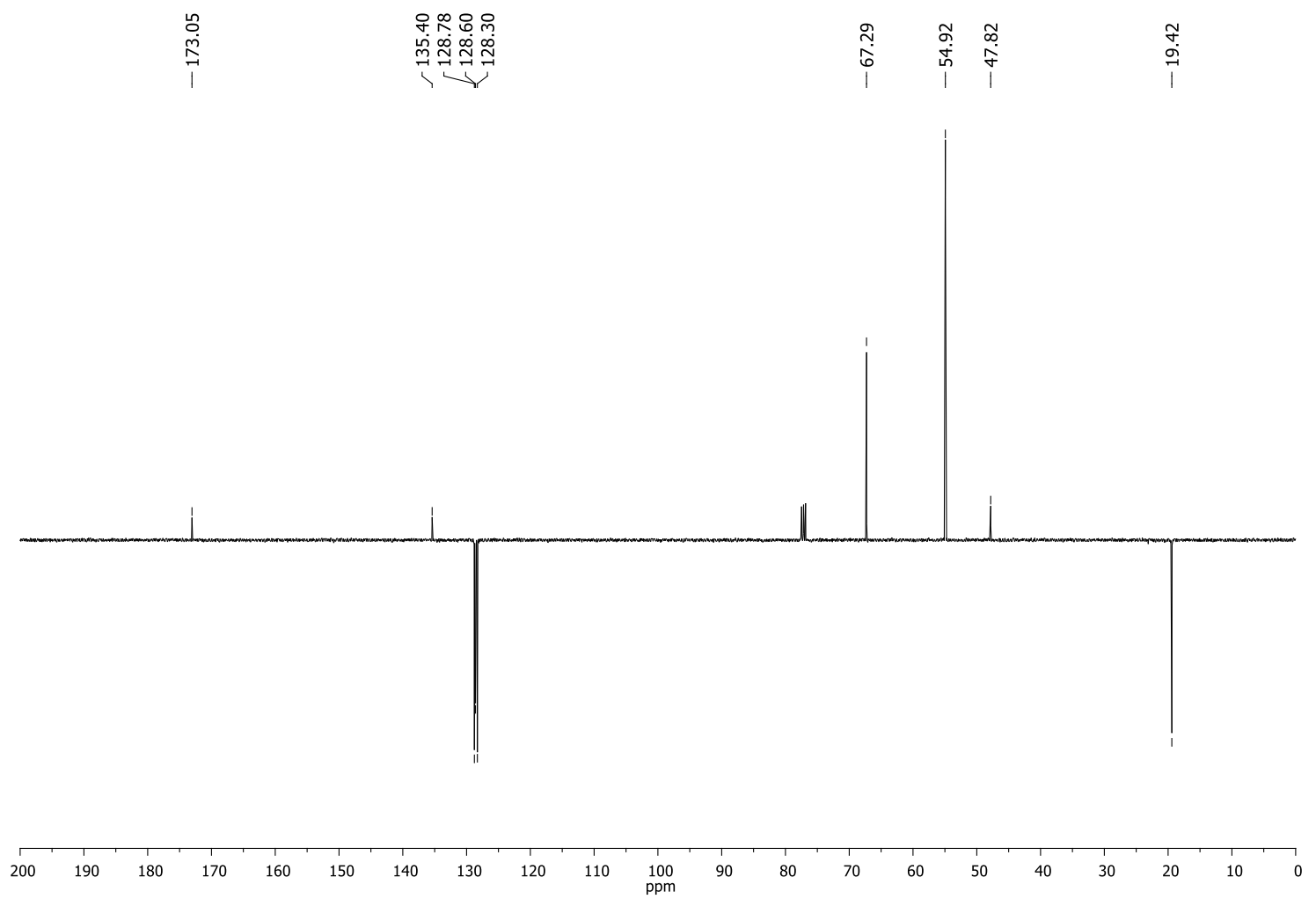

Figure S4. ${ }^{13} \mathrm{C}$ NMR (SEFT) spectrum of benzyl-3,3'-diazidopivaloate in $\mathrm{CDCl}_{3}$. 


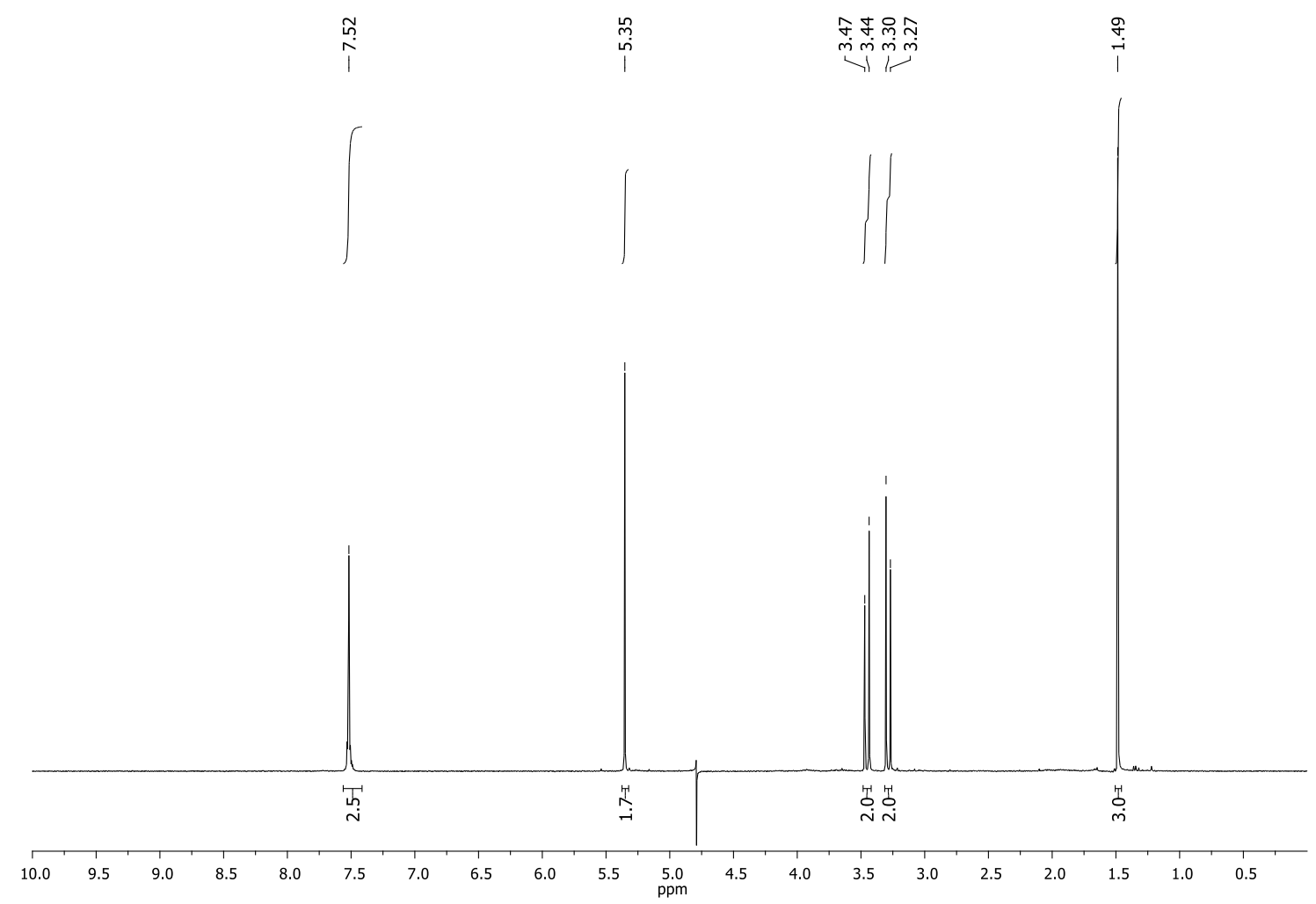

Figure S5. ${ }^{1} \mathrm{H}$ NMR spectrum of benzyl-3,3'-diaminopivaloate in $\mathrm{D}_{2} \mathrm{O}$.

\begin{tabular}{|c|c|c|c|}
\hline$\underset{\sim ָ}{\tilde{N}}$ & 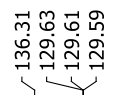 & $\begin{array}{l}\hat{m} \\
\text { o. } \\
1\end{array}$ & 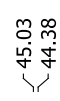 \\
\hline
\end{tabular}

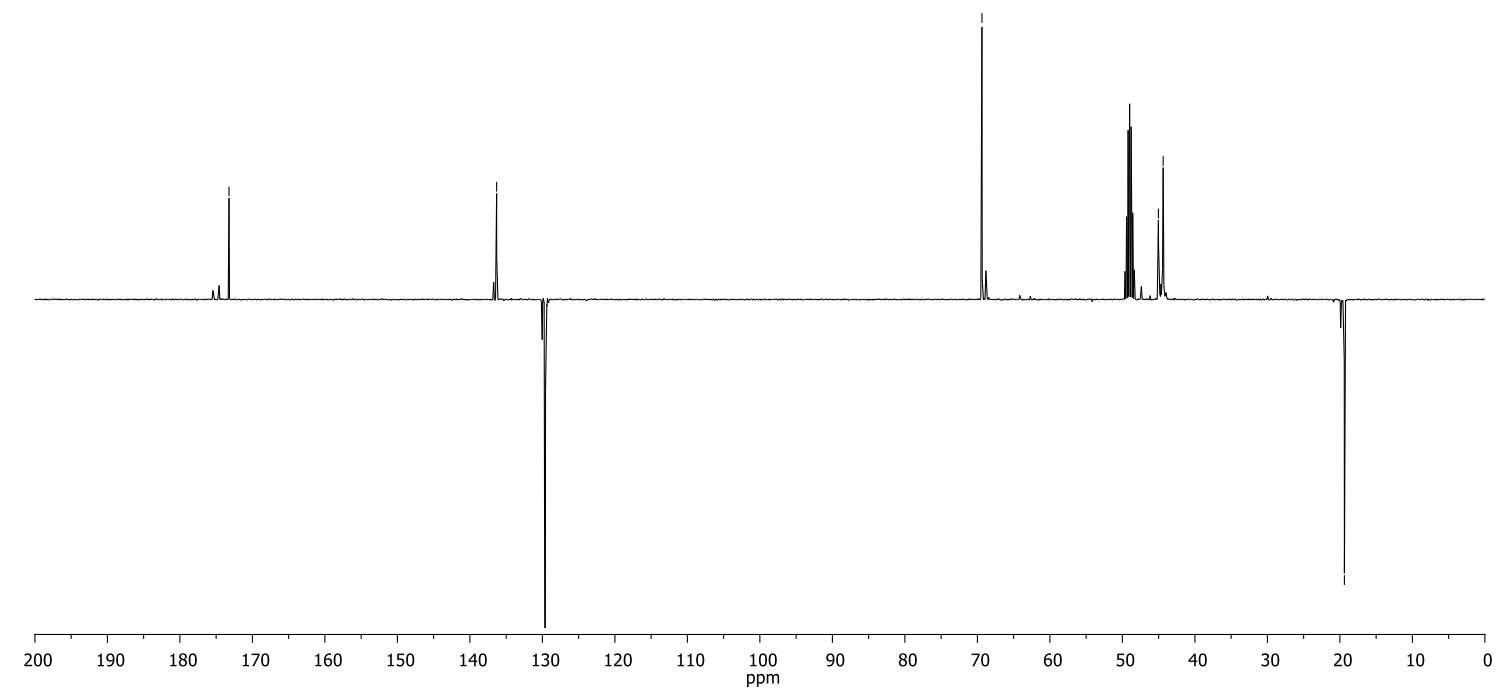

Figure S6. ${ }^{13} \mathrm{C}$ NMR (SEFT) spectrum of benzyl-3,3'-diaminopivaloate in $\mathrm{D}_{2} \mathrm{O}$. 


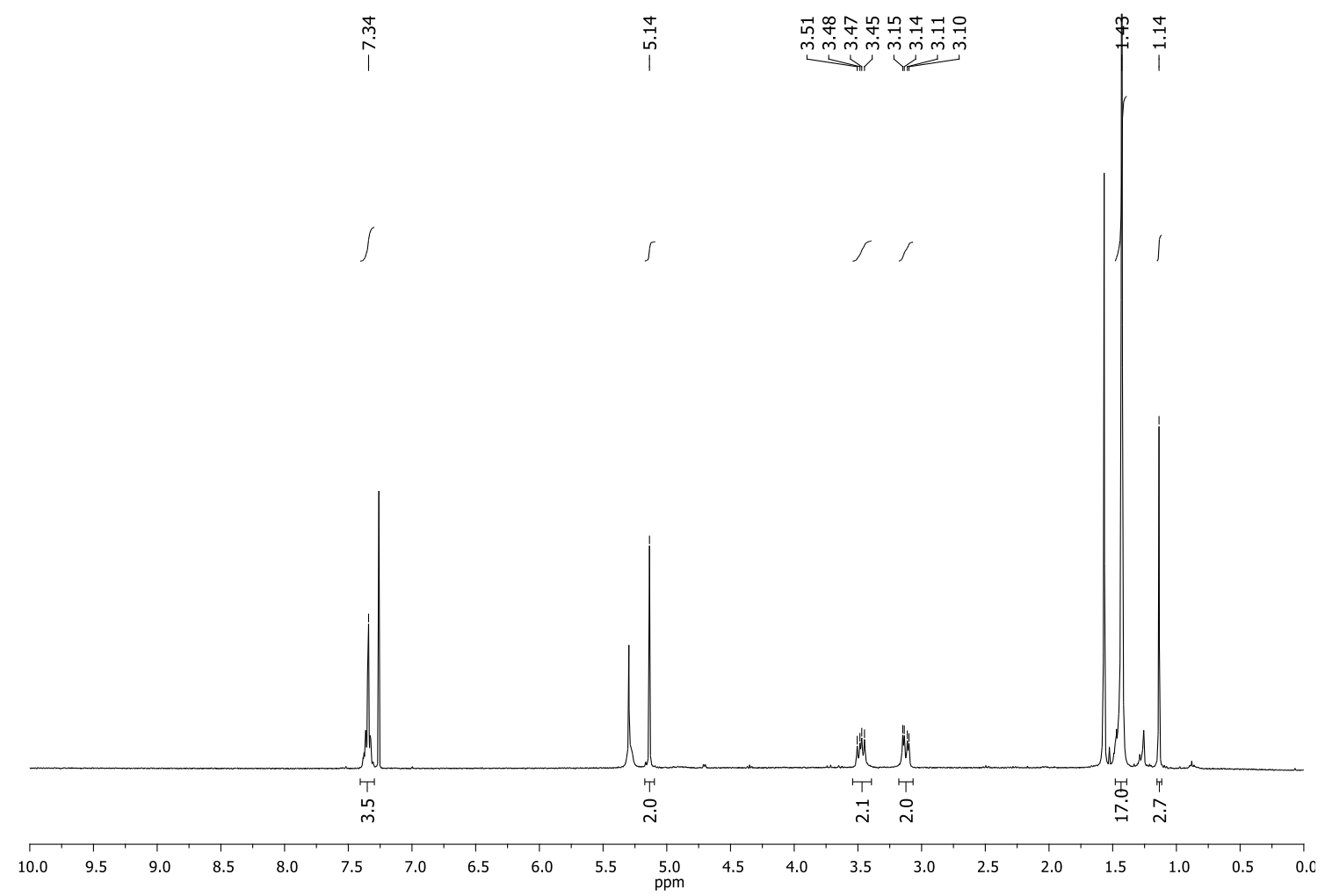

Figure S7. ${ }^{1} \mathrm{H}$ NMR spectrum of benzyl-3,3'-bis(tert-butoxycarbonyl)aminopivaloate in $\mathrm{CDCl}_{3}$.

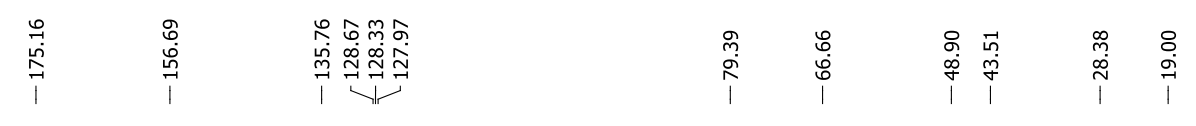

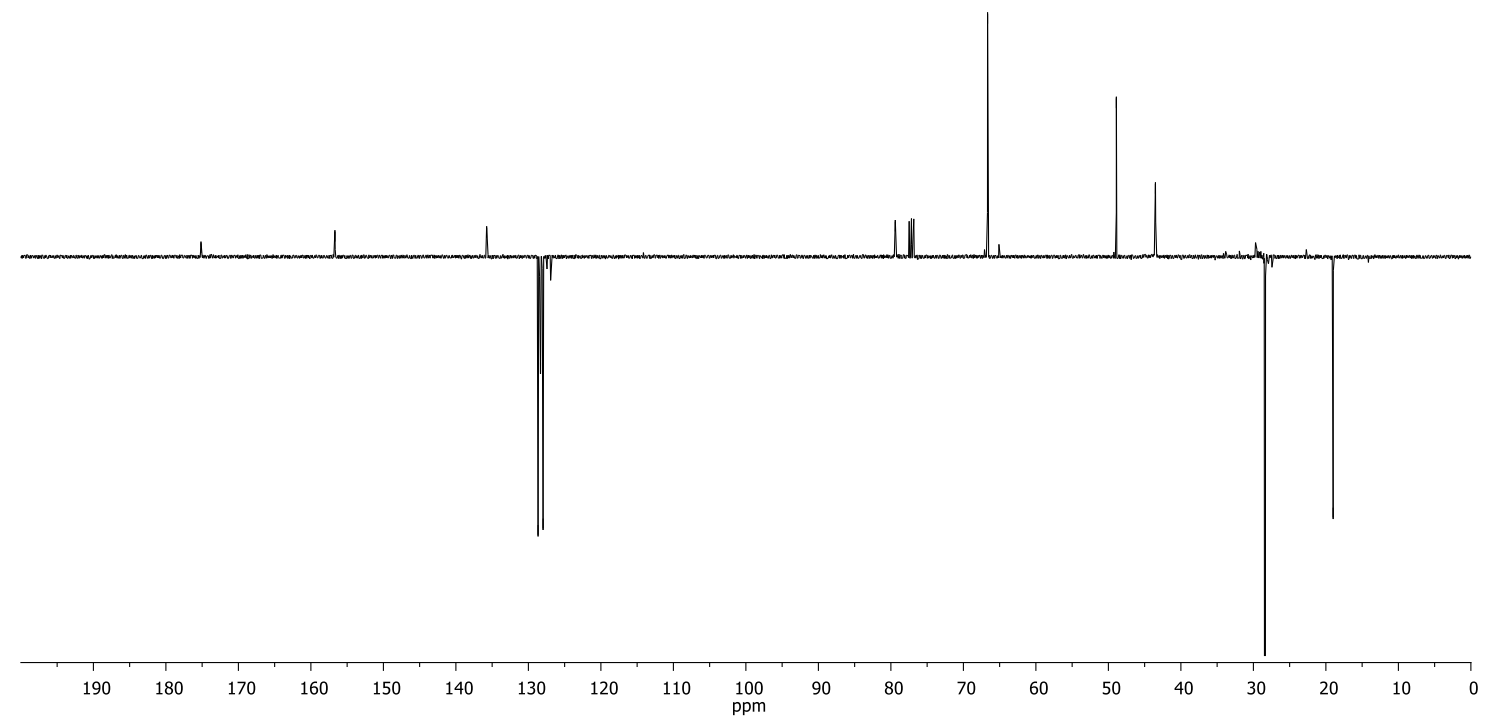

Figure S8. ${ }^{13} \mathrm{C}$ NMR (SEFT) spectrum of benzyl-3,3'-bis(tert-butoxycarbonyl)aminopivaloate in $\mathrm{CDCl}_{3}$. 


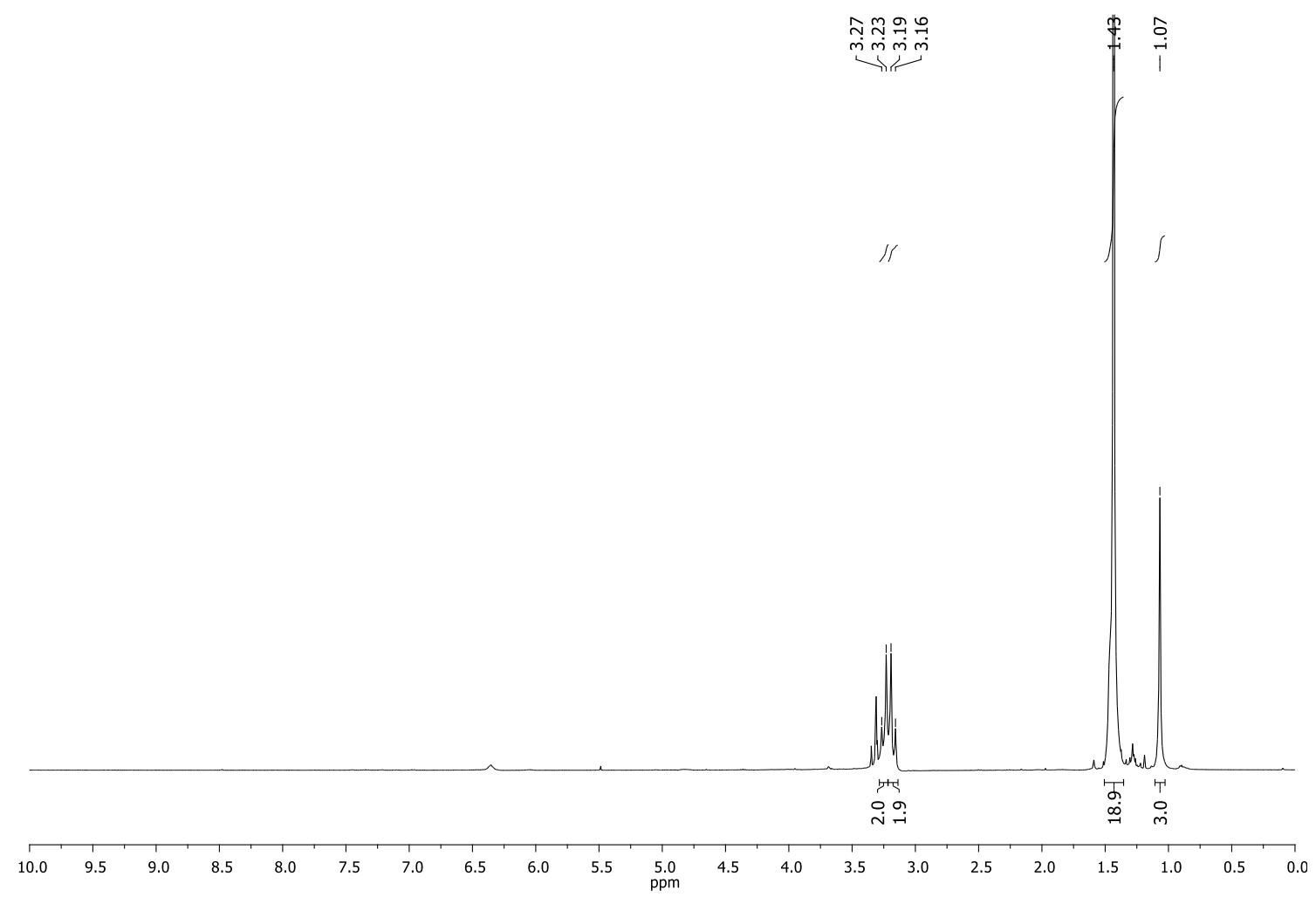

Figure S9. ${ }^{1} \mathrm{H}$ NMR spectrum of 3,3'-bis(tert-butoxycarbonyl)aminopivalic acid in MeOD- $d_{4}$.

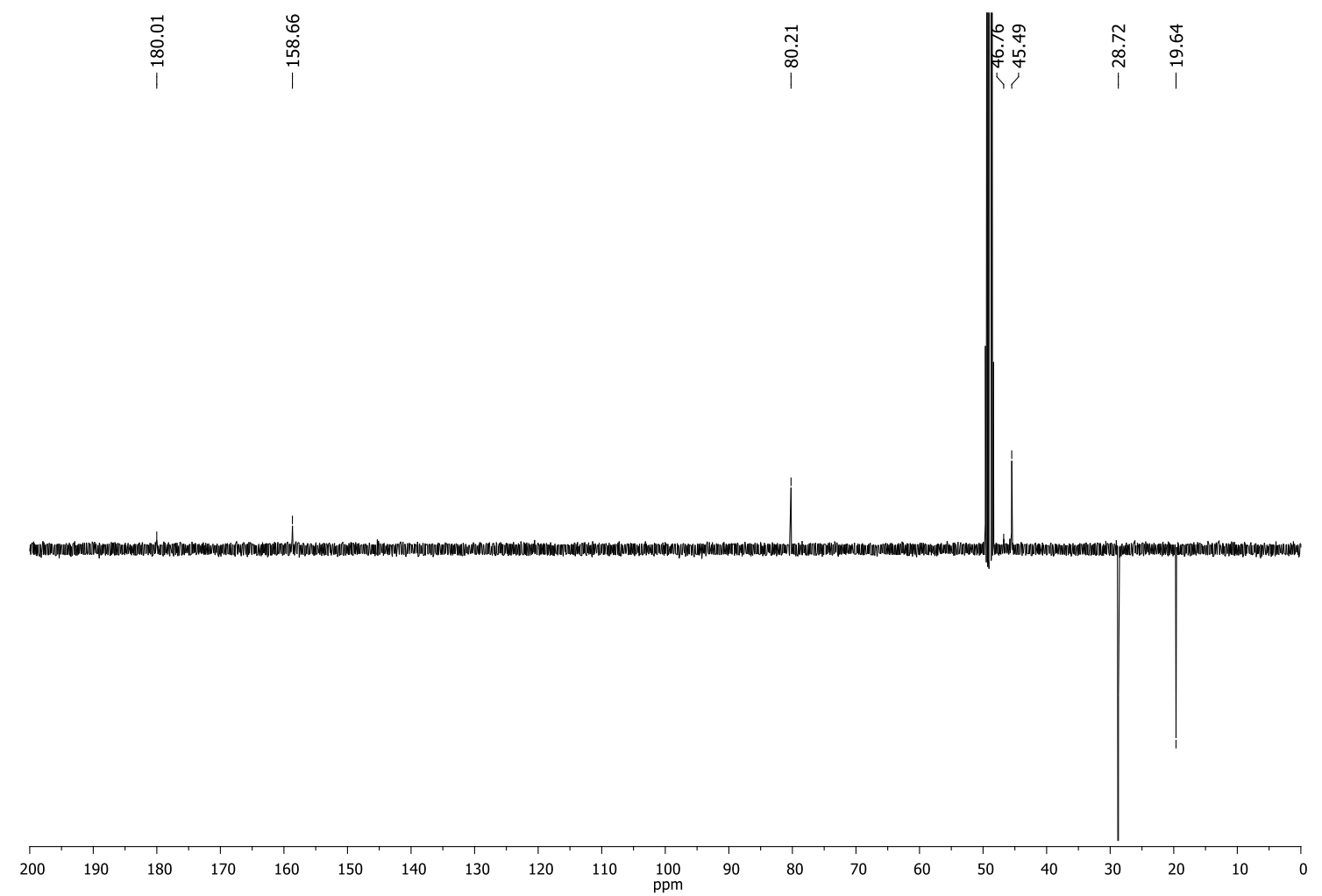

Figure S10. ${ }^{13} \mathrm{C}$ NMR (SEFT) spectrum of 3,3'-bis(tert-butoxycarbonyl)aminopivalic acid in MeOD- $d 4$. 


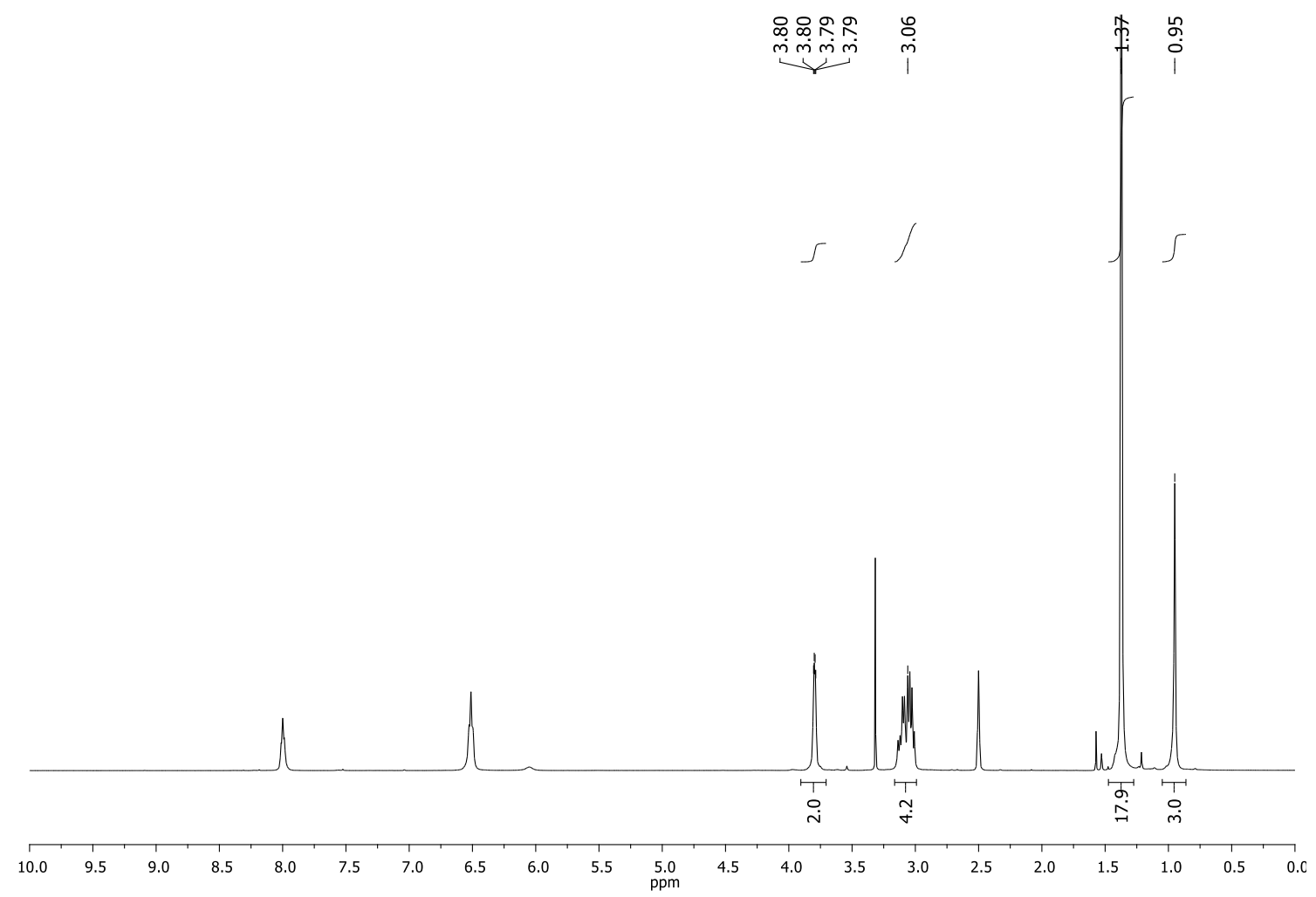

Figure S11. ${ }^{1} \mathrm{H}$ NMR spectrum of dG1 in DMSO- $d 6$.

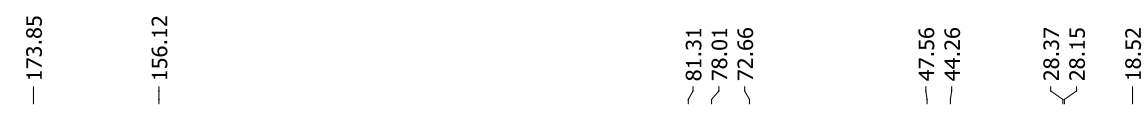

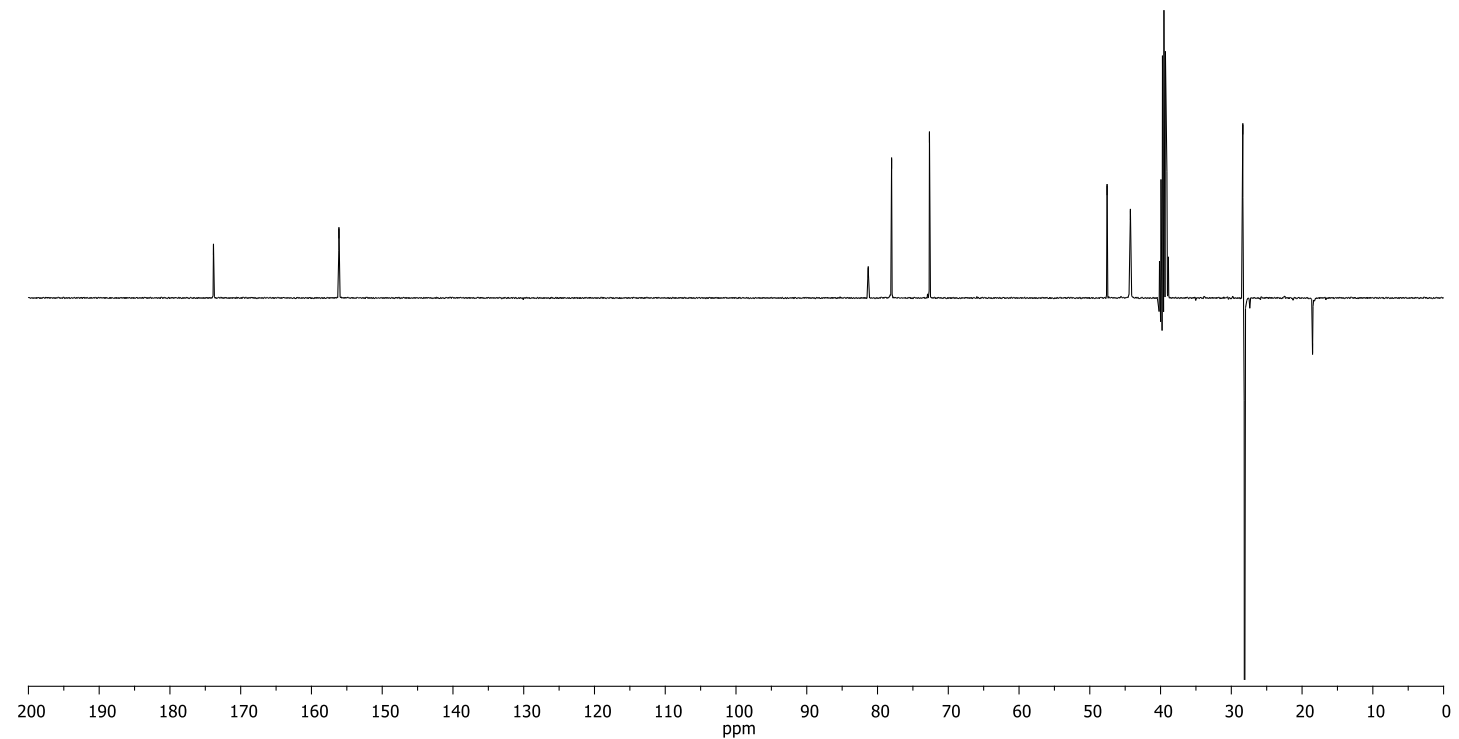

Figure S12. ${ }^{13} \mathrm{C}$ NMR (SEFT) spectrum of dG1 in DMSO- $d 6$. 


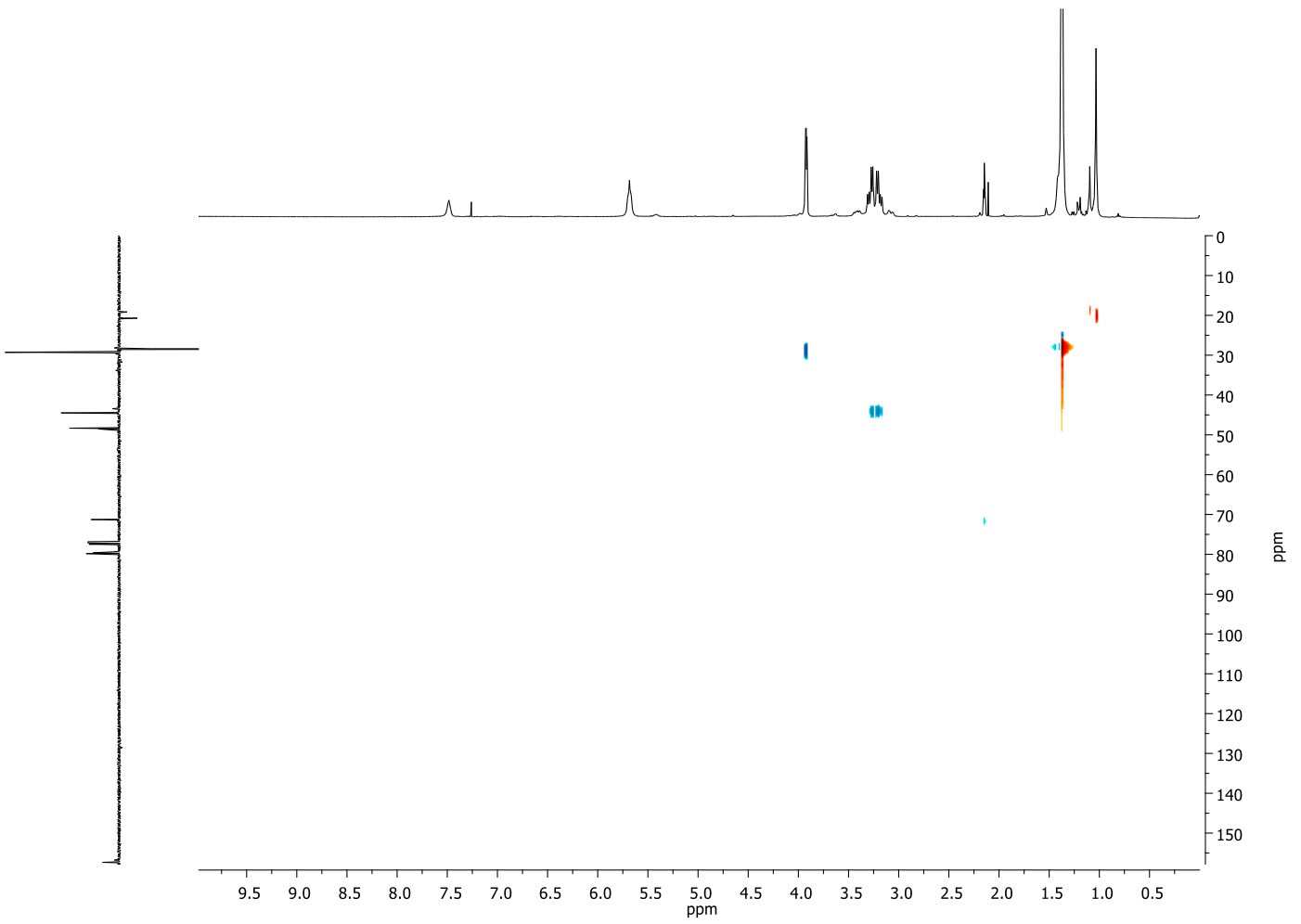

Figure S13. HSQC spectrum of dG1 in DMSO- $d 6$. 


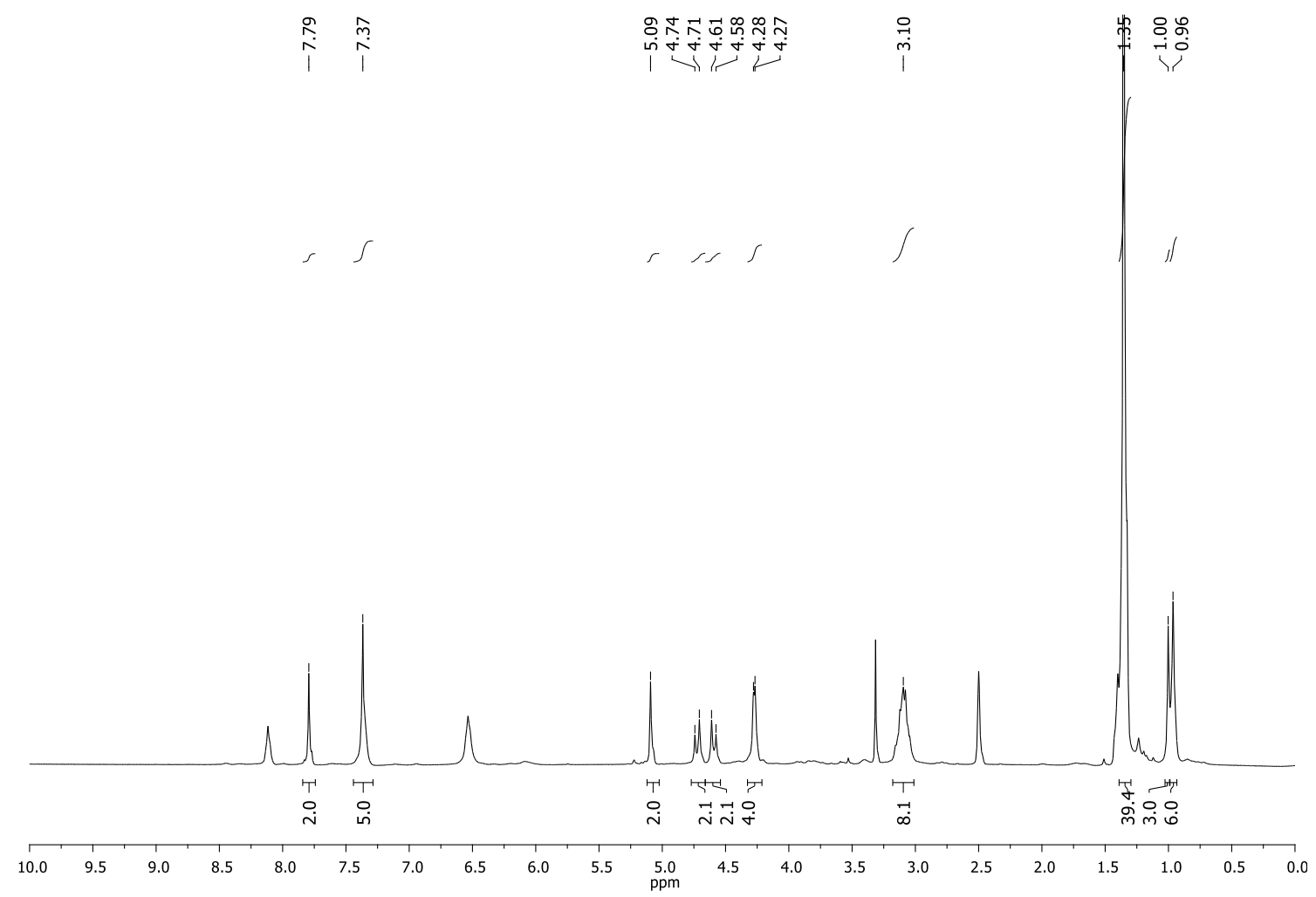

Figure S14. ${ }^{1} \mathrm{H}$ NMR spectrum of $\mathbf{d G 2}-\mathbf{C O}_{2} \mathbf{B n}$ in DMSO- $d_{6}$.
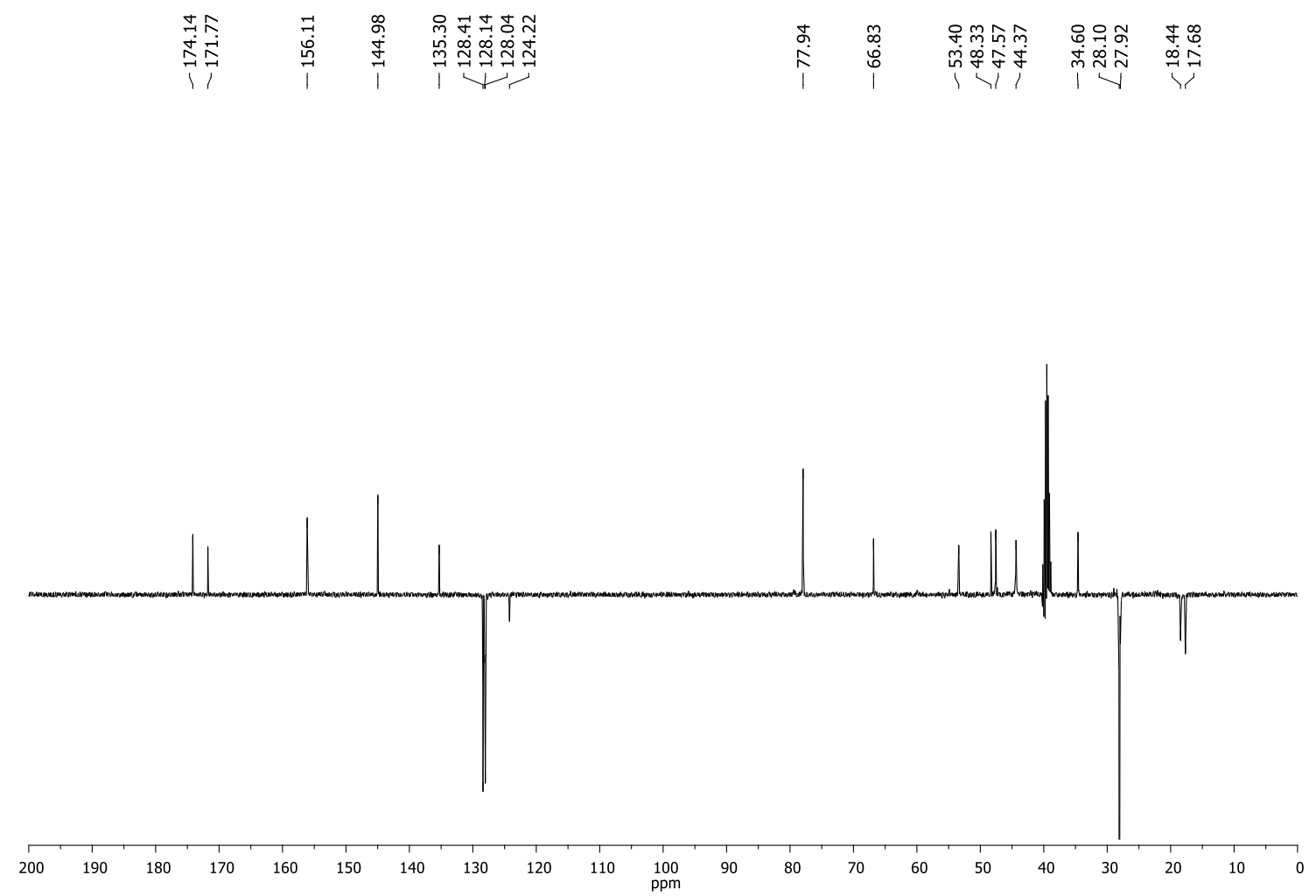

Figure S15. ${ }^{13} \mathrm{C}$ NMR (SEFT) spectrum of dG2-CO 2 Bn in DMSO- $d_{6}$. 


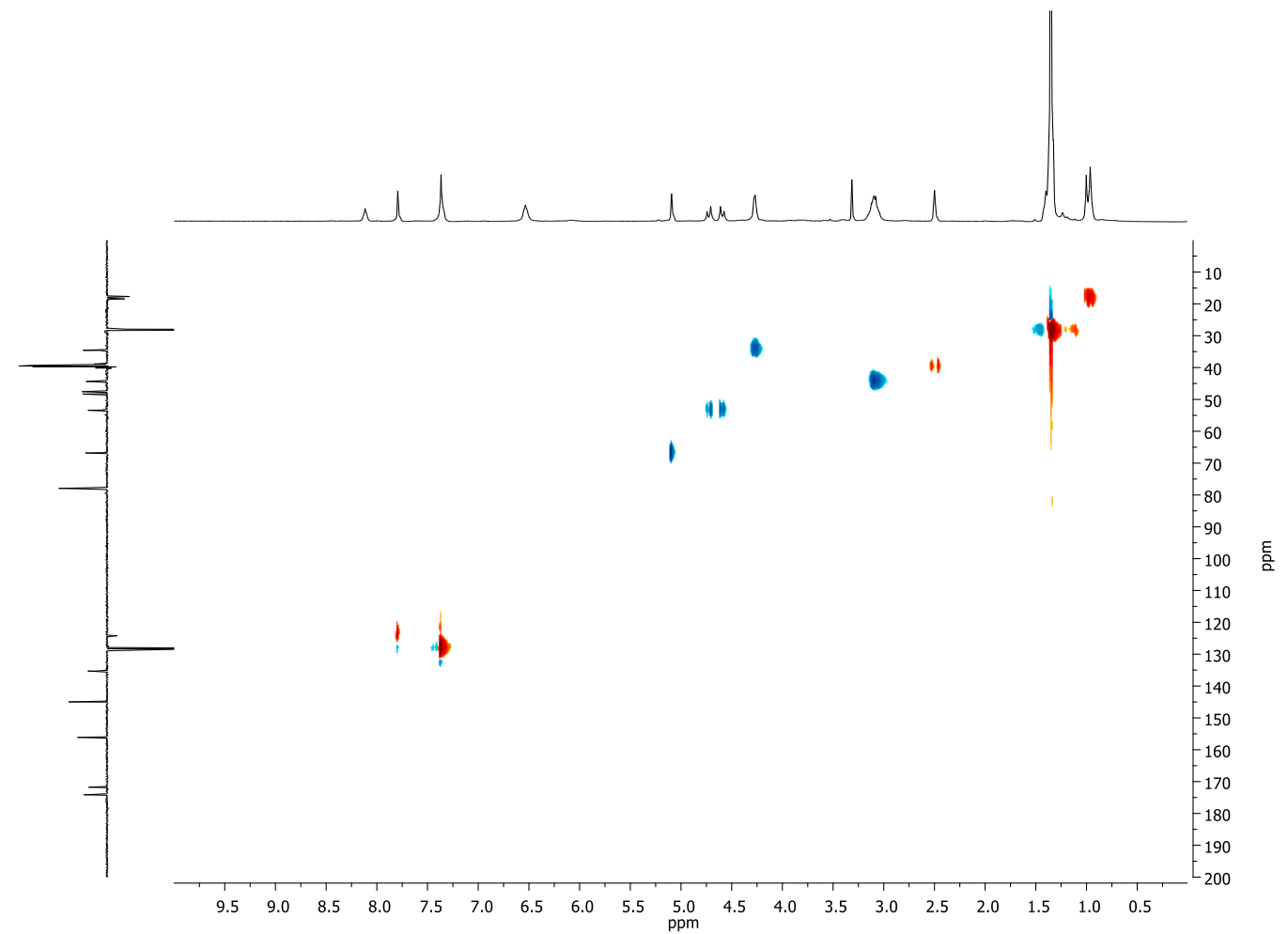

Figure S16. HSQC spectrum of dG2- $\mathrm{CO}_{2} \mathrm{Bn}$ in DMSO- $d 6$. 


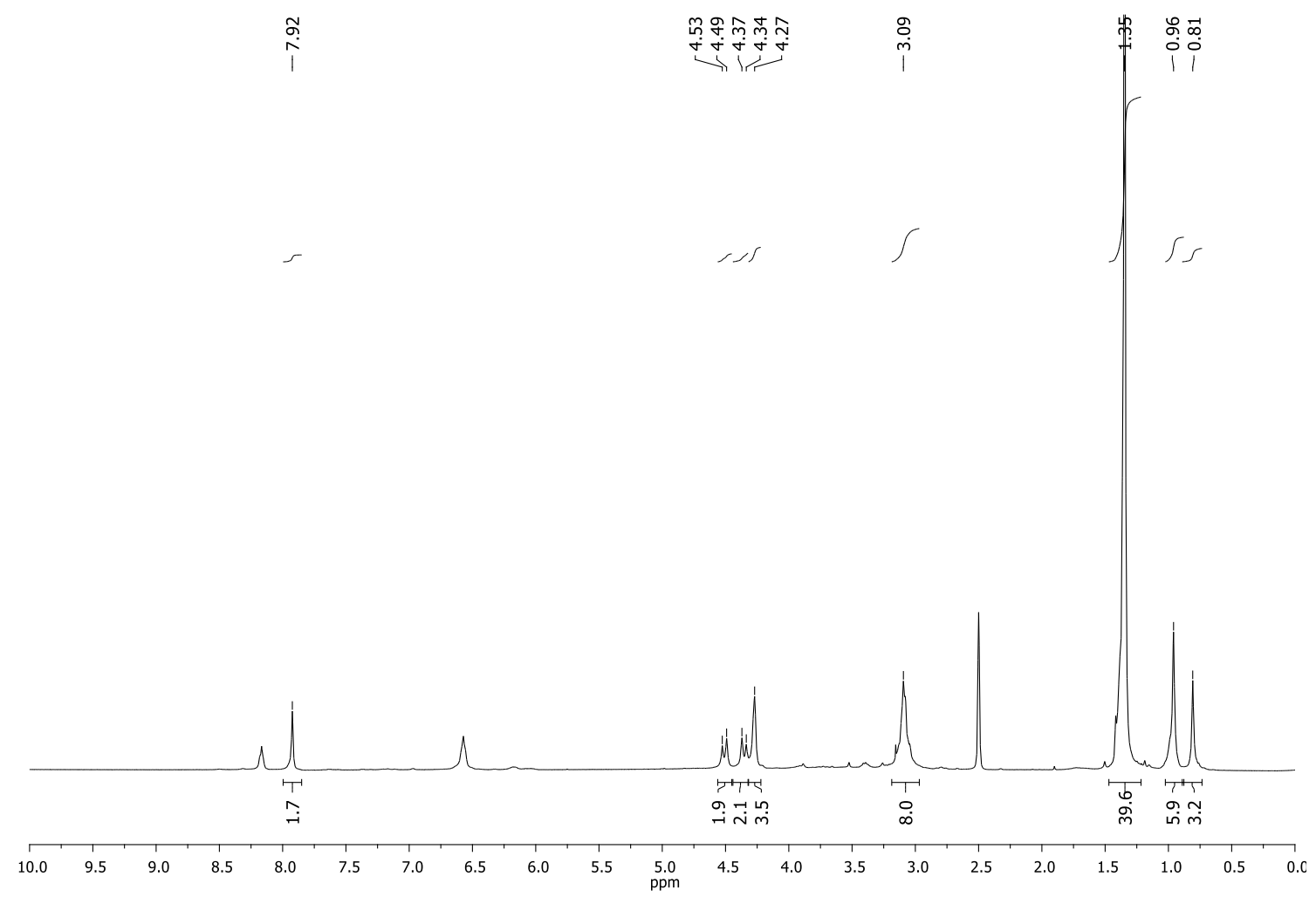

Figure S17. ${ }^{1} \mathrm{H}$ NMR spectrum of dG2-CO $2 \mathbf{H}$ in DMSO-d6.

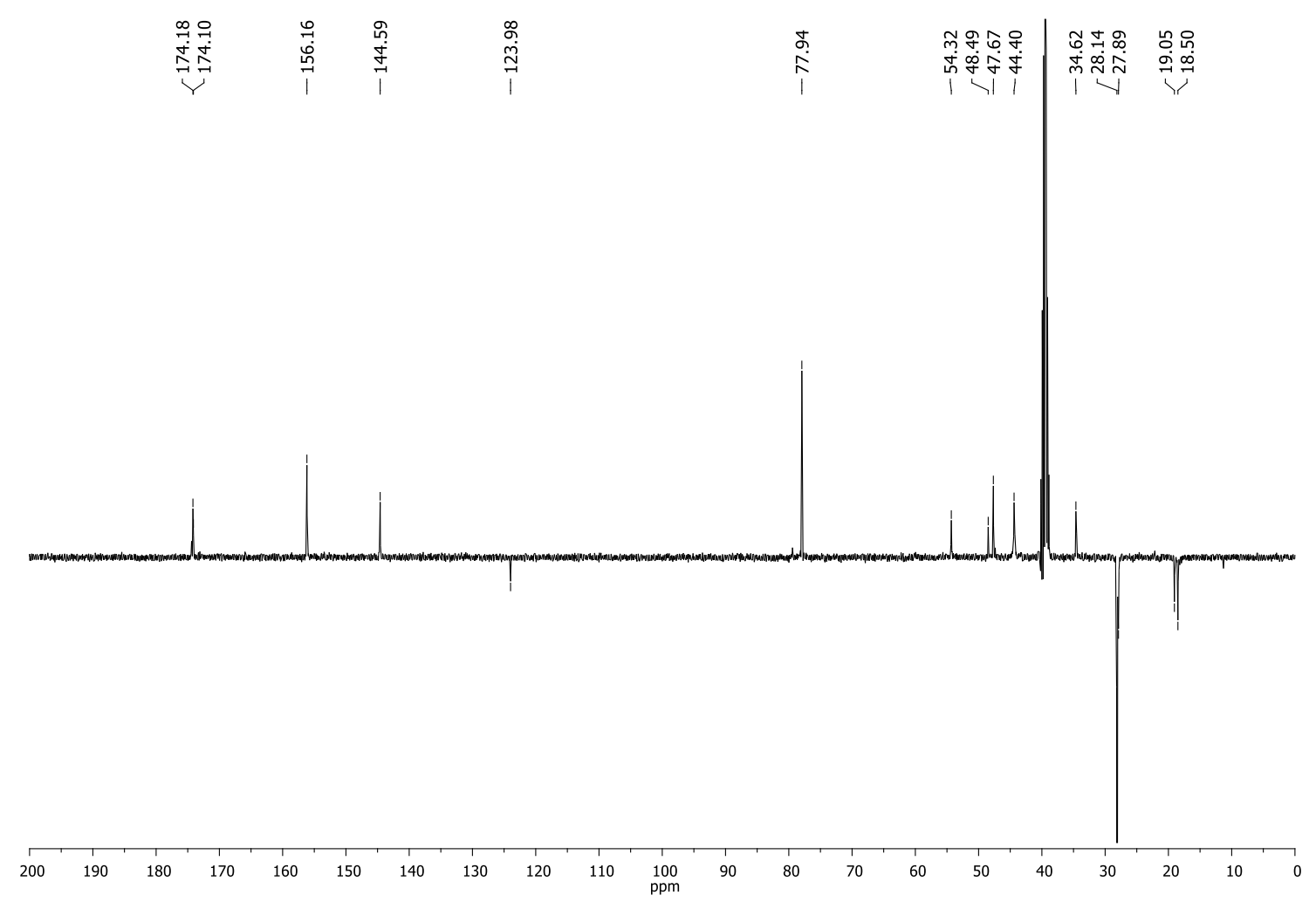

Figure S18. ${ }^{13} \mathrm{C}$ NMR (SEFT) spectrum of $\mathbf{d G 2}-\mathbf{C O}_{2} \mathbf{H}$ in DMSO- $d_{6}$. 


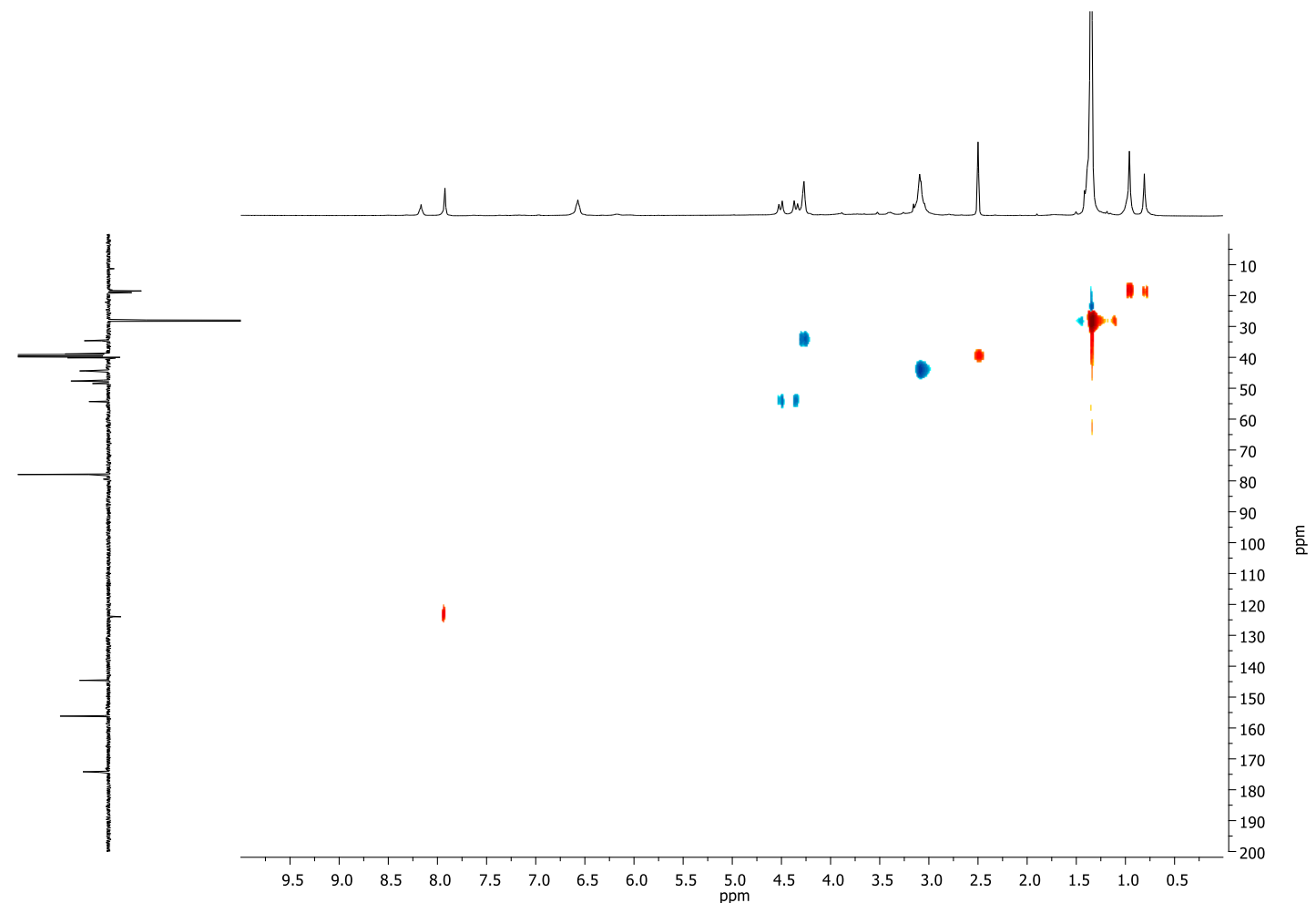

Figure S19. HSQC spectrum of dG2- $\mathrm{CO}_{2} \mathrm{H}$ in DMSO- $d 6$. 


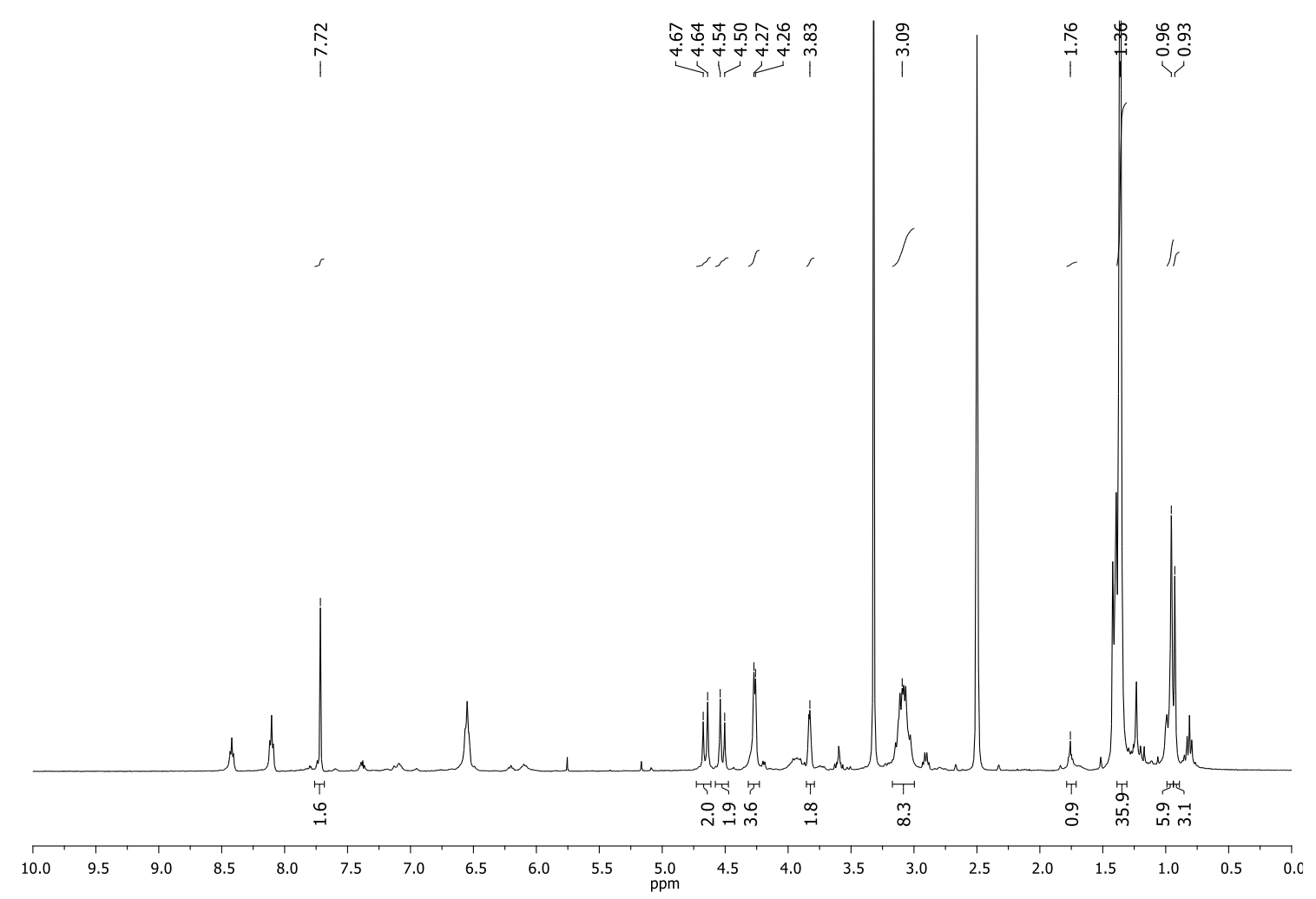

Figure S20. ${ }^{1} \mathrm{H}$ NMR spectrum of dG2 in DMSO- $d 6$.

辛紊

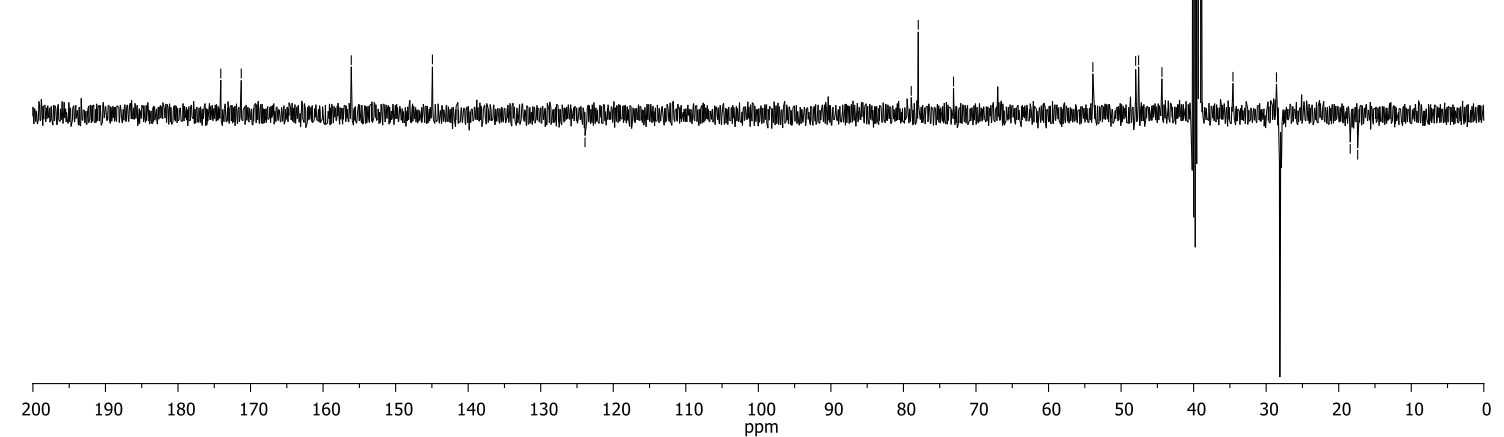

Figure S21. ${ }^{13} \mathrm{C}$ NMR (SEFT) spectrum of dG2 in DMSO- $d 6$. 


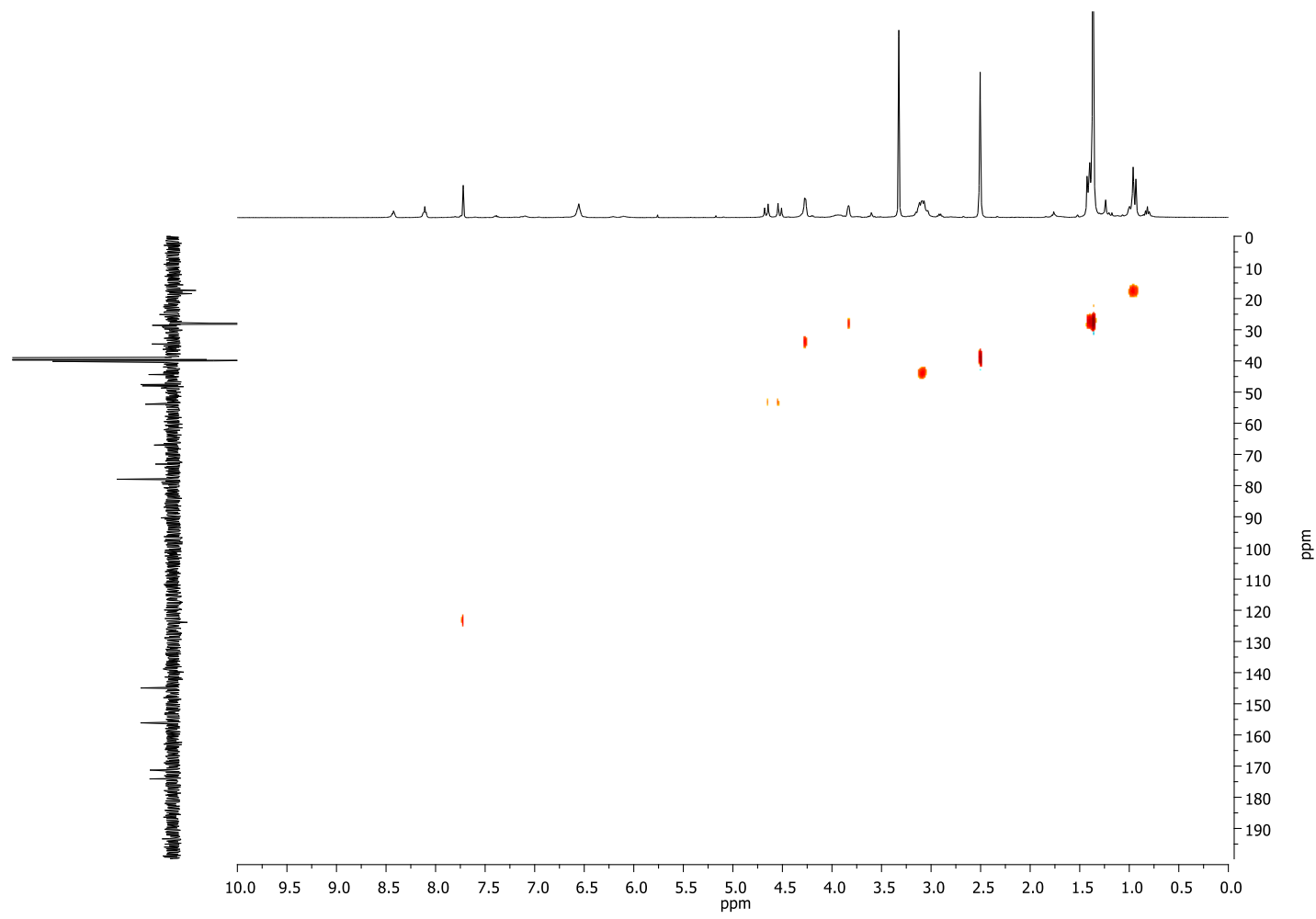

Figure S22. HSQC spectrum of dG2 in DMSO- $d 6$.

S-14 


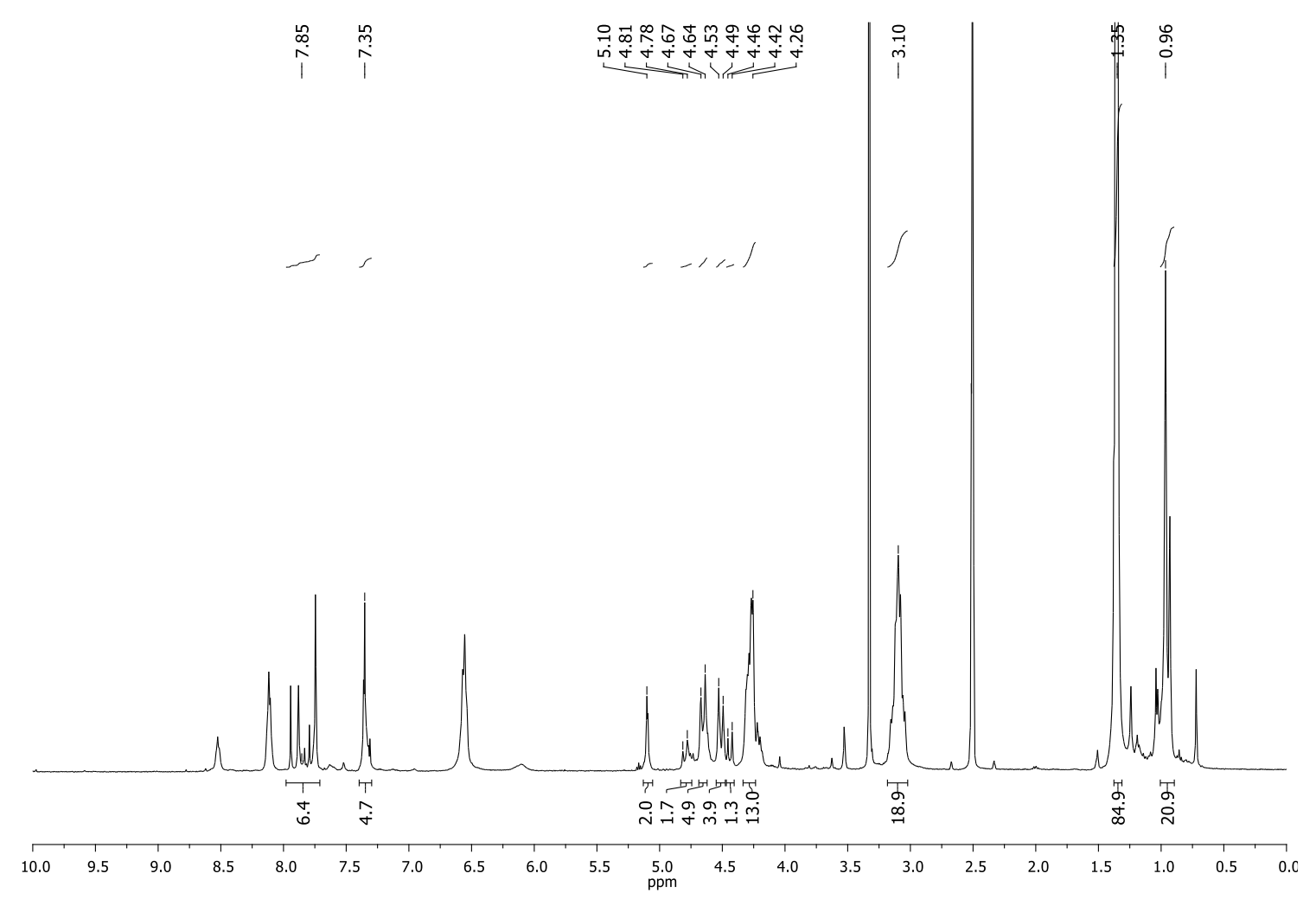

Figure S23. ${ }^{1} \mathrm{H}$ NMR spectrum of dG3-COO $2 \mathbf{B n}$ in DMSO-d $d_{6}$.

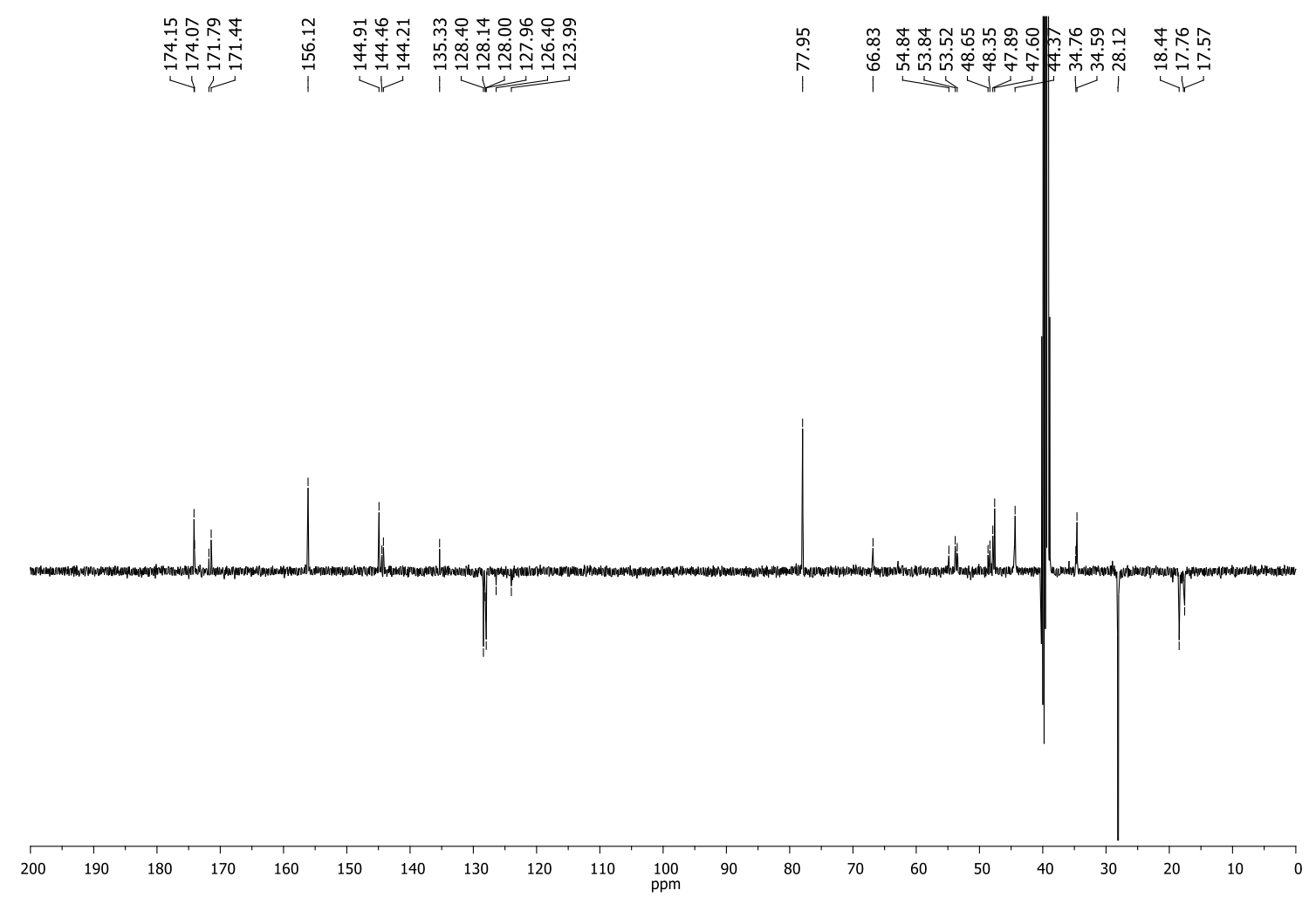

Figure S24. ${ }^{13} \mathrm{C}$ NMR (SEFT) spectrum of dG3- $\mathbf{C O}_{2} \mathbf{B n}$ in DMSO- $d_{6}$. 


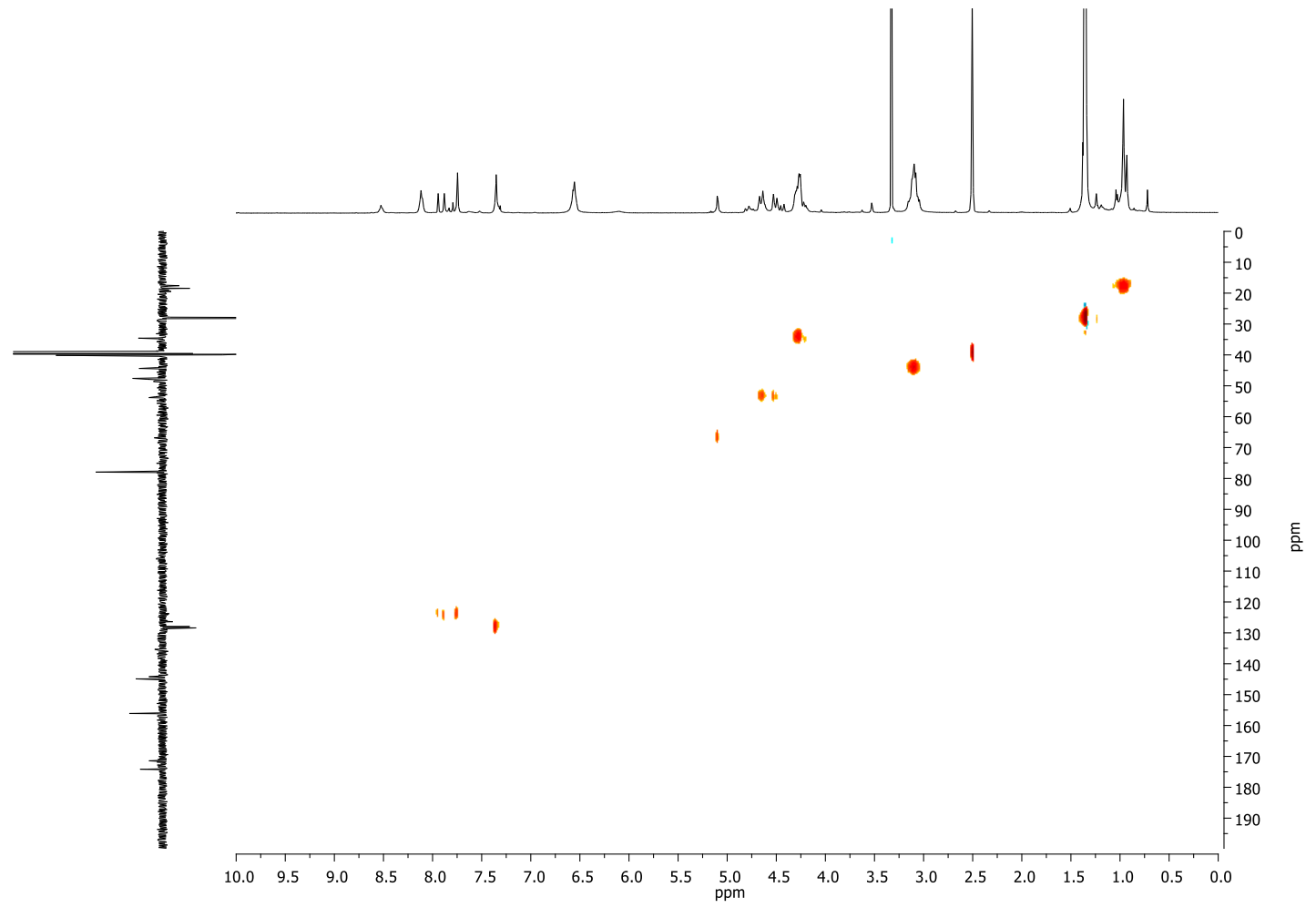

Figure S25. HSQC spectrum of dG3-CO2 $\mathbf{B n}$ in DMSO- $d 6$. 


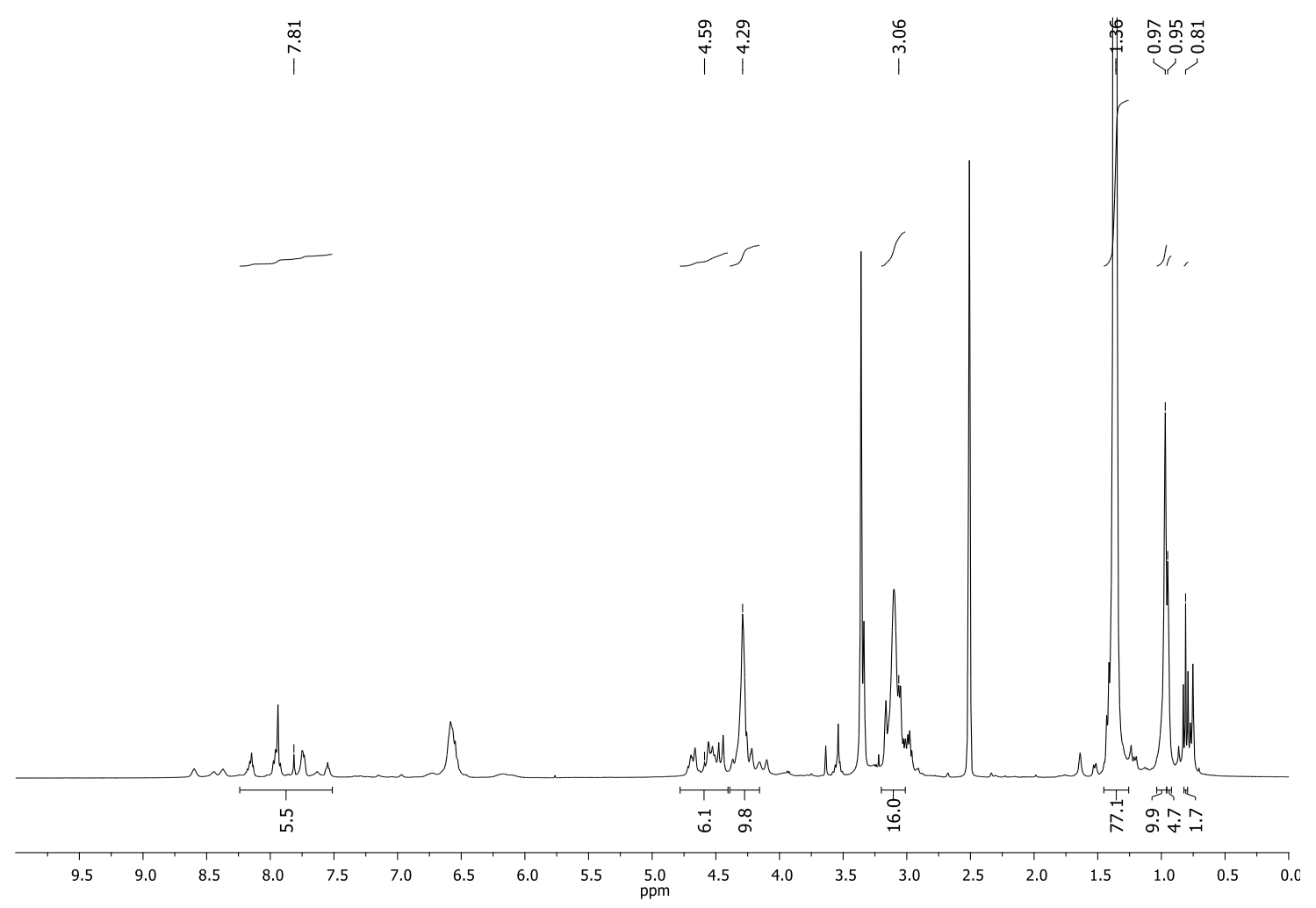

Figure S26. ${ }^{1} \mathrm{H}$ NMR spectrum of dG3-CO $2 \mathbf{H}$ in DMSO- $d_{6}$.

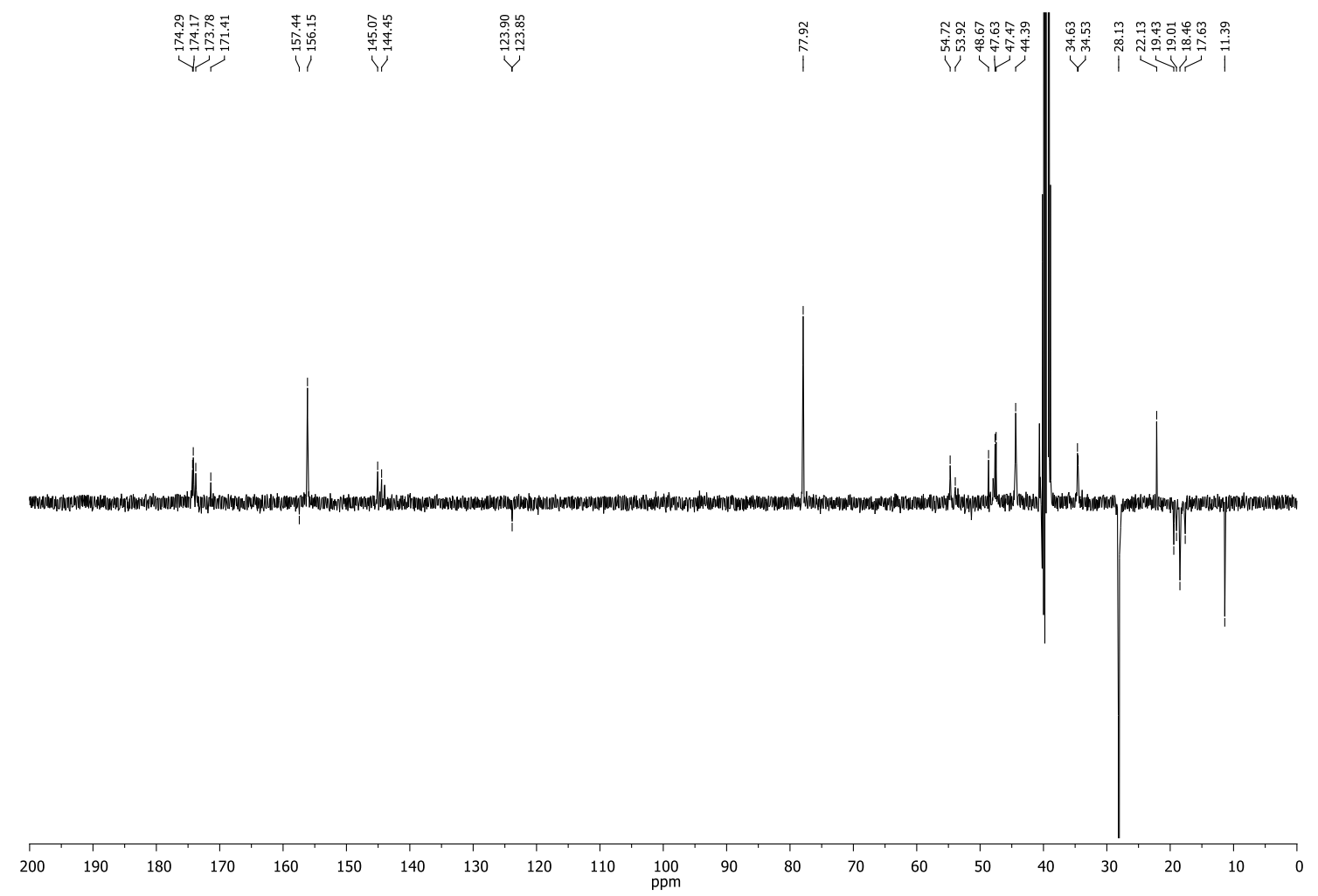

Figure S27. ${ }^{13} \mathrm{C}$ NMR (SEFT) spectrum of dG3-CO $2 \mathbf{H}$ in DMSO-d $d_{6}$ 


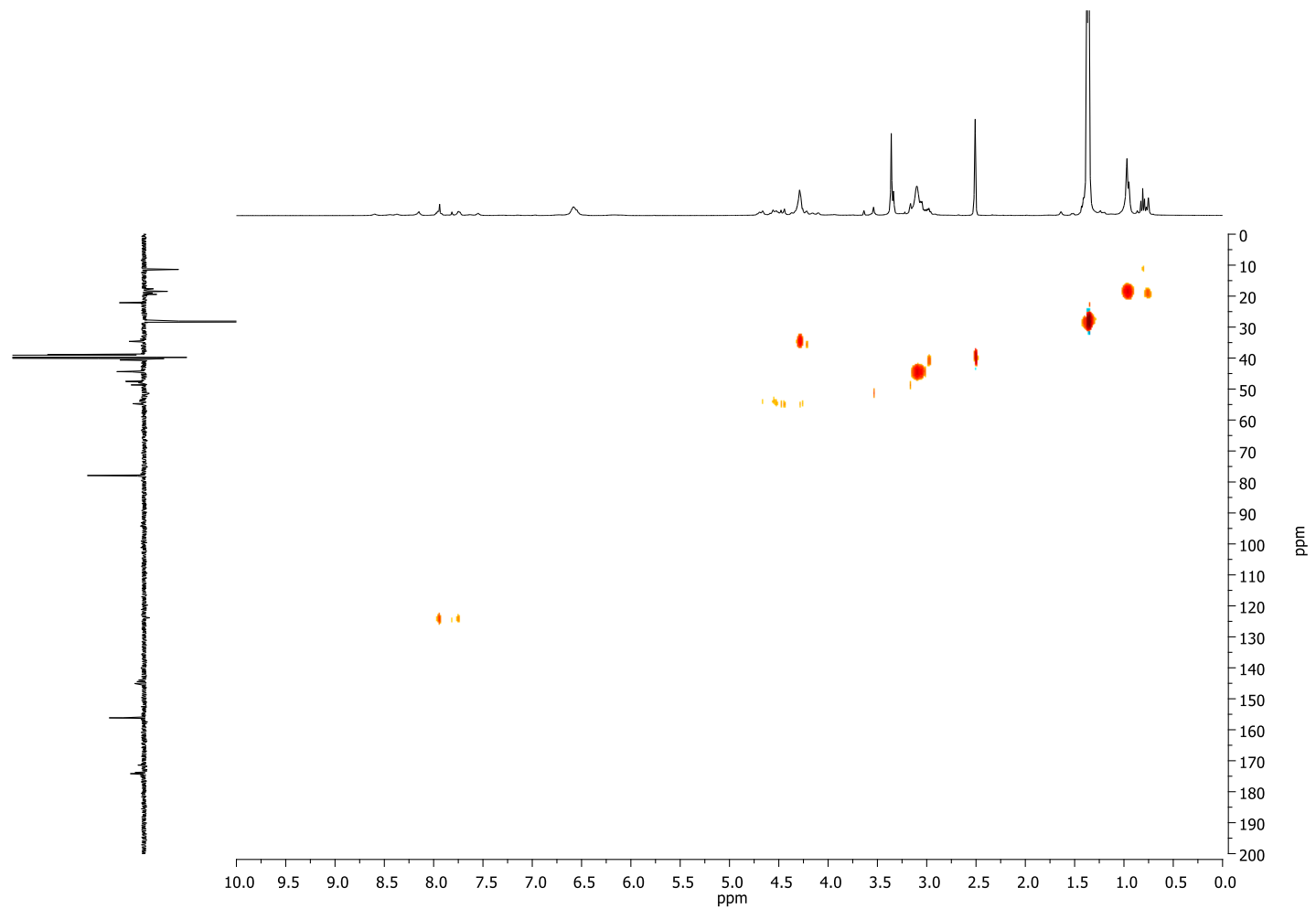

Figure S28. HSQC spectrum of dG3- $\mathrm{CO}_{2} \mathrm{H}$ in DMSO- $d_{6}$. 


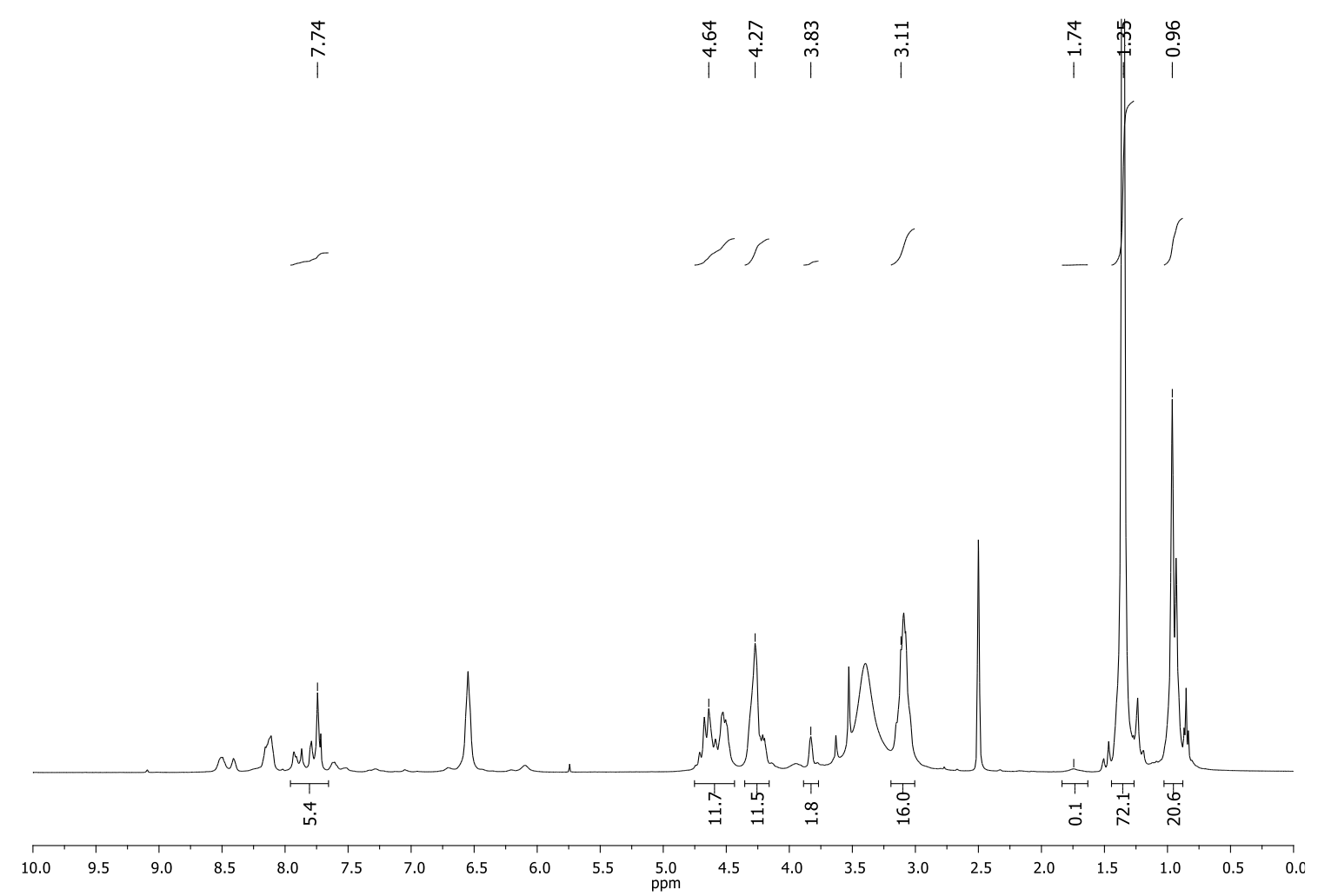

Figure S29. ${ }^{1} \mathrm{H}$ NMR spectrum of $\mathbf{d G 3}$ in DMSO- $d_{6}$.

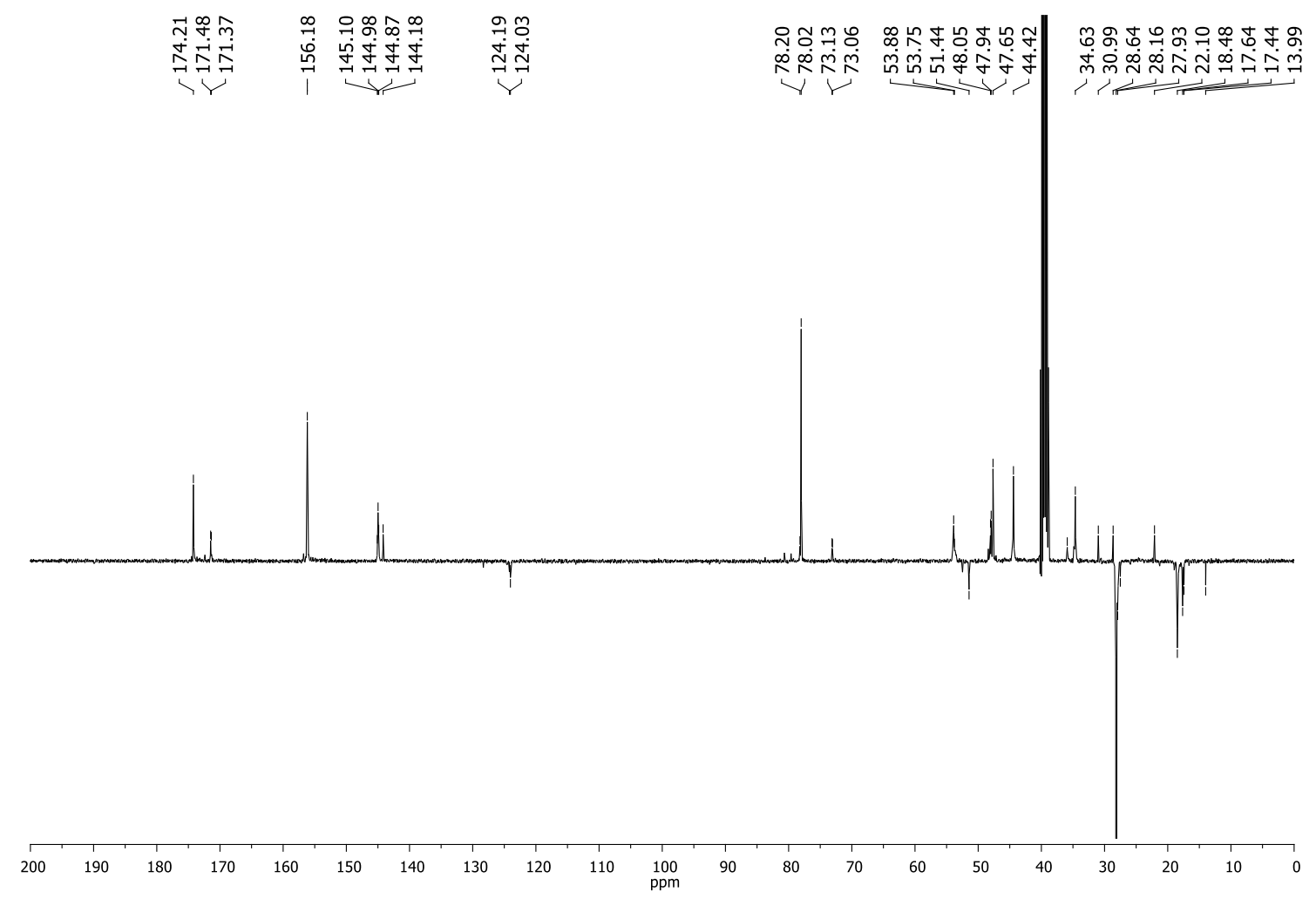

Figure S30. ${ }^{13} \mathrm{C}$ NMR (SEFT) spectrum of dG3 in DMSO- $d_{6}$. 


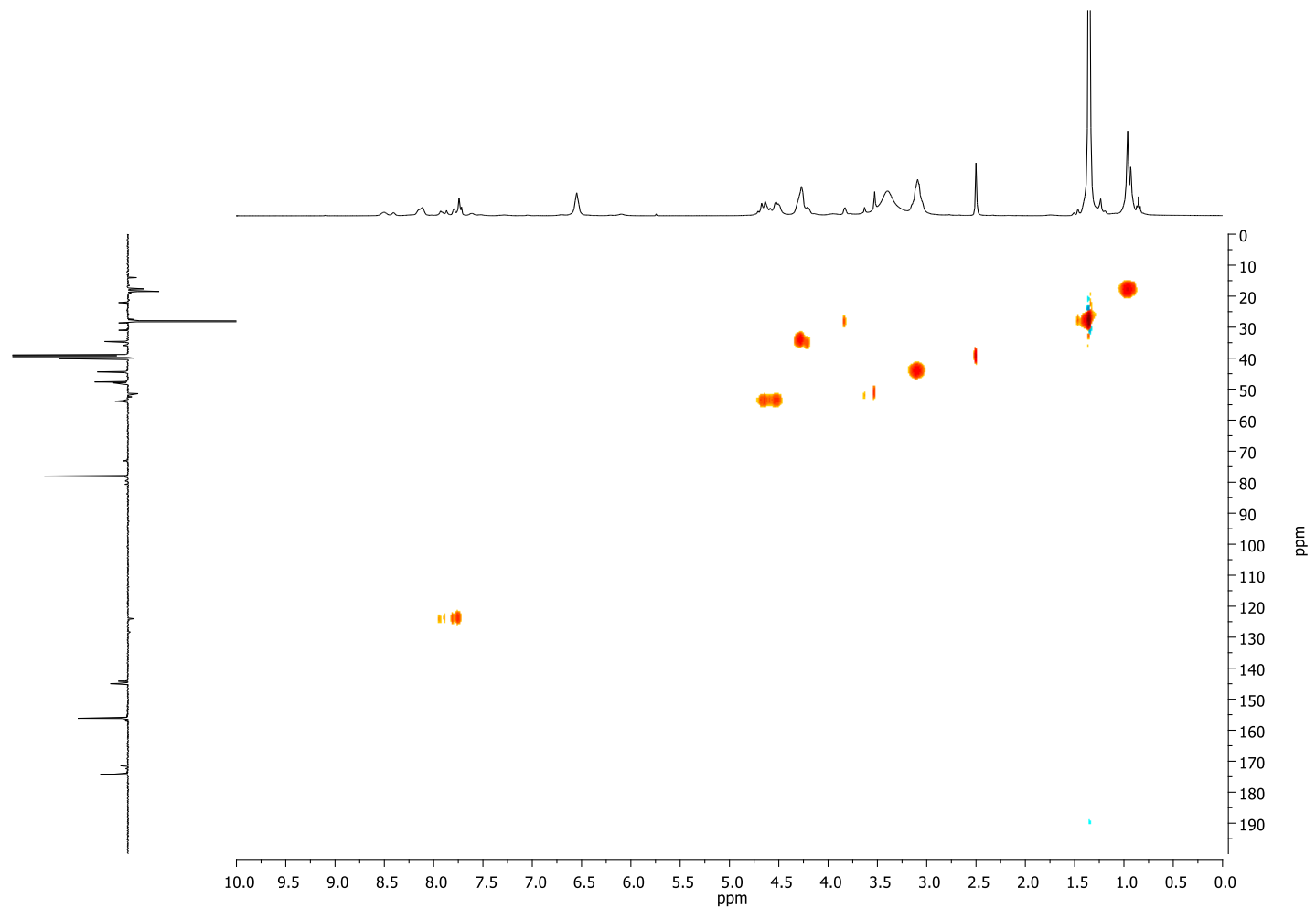

Figure S31. HSQC spectrum of dG3 in DMSO- $d_{6}$. 


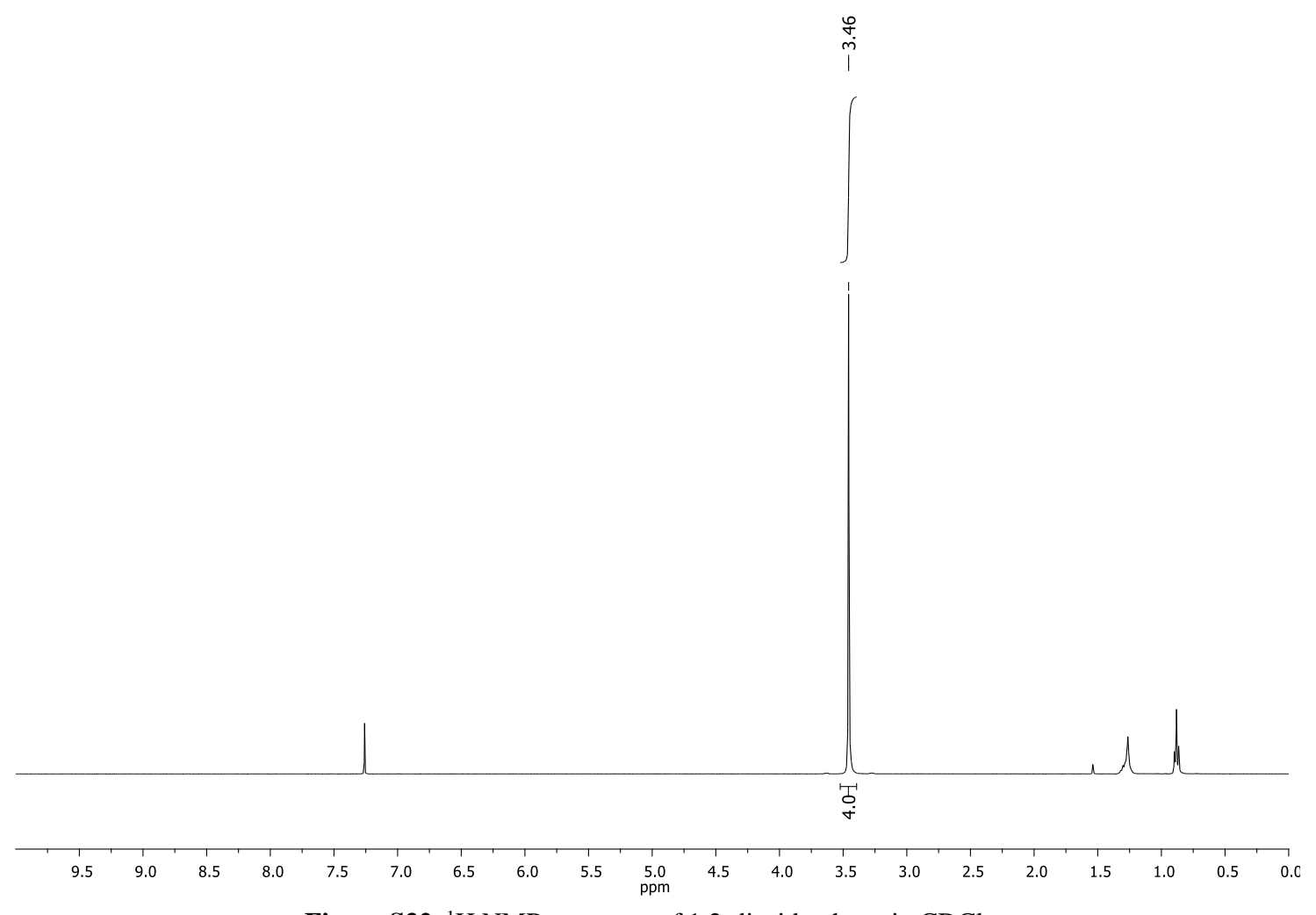

Figure S32. ${ }^{1} \mathrm{H}$ NMR spectrum of 1,2-diazidoethane in $\mathrm{CDCl}_{3}$. 


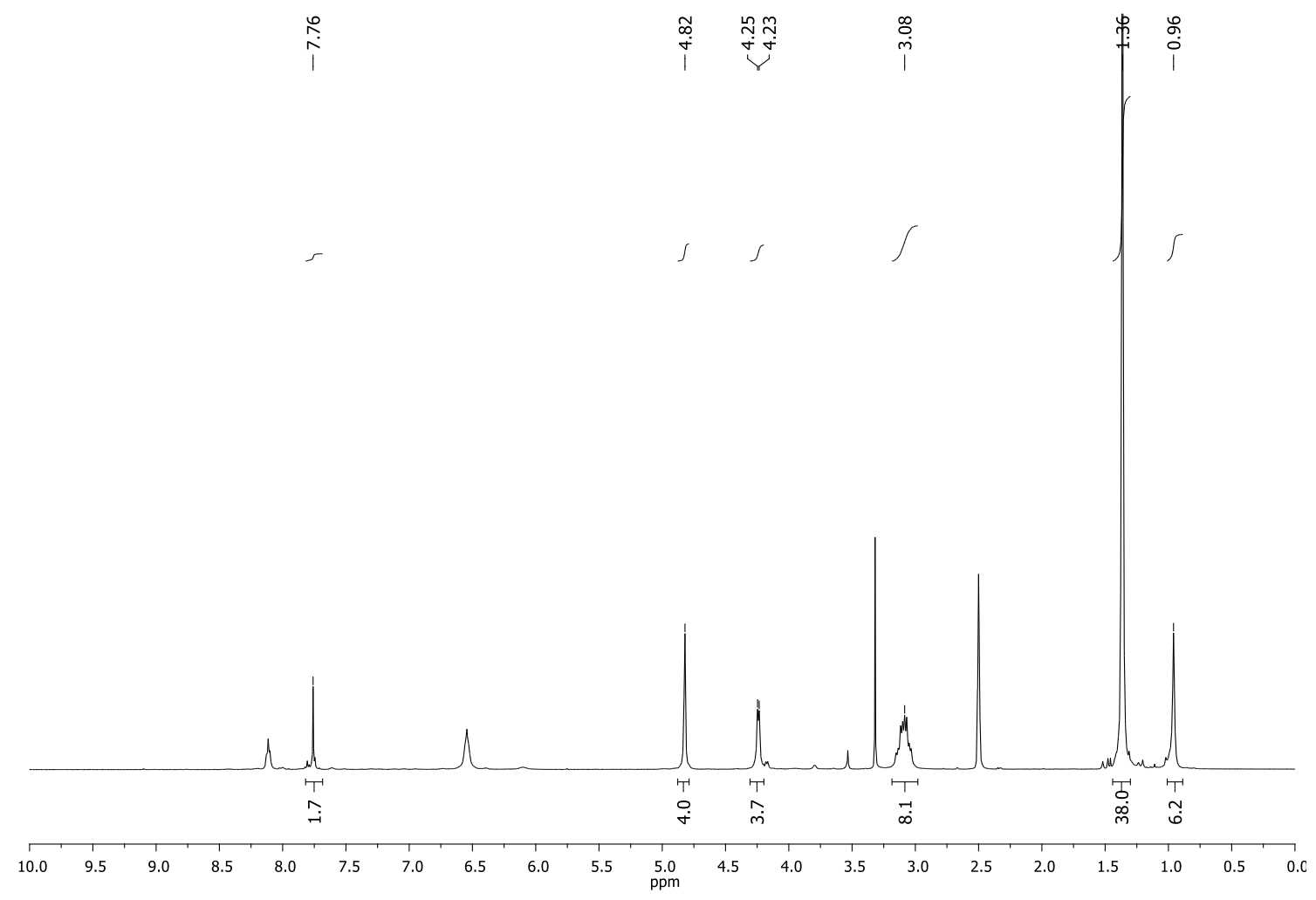

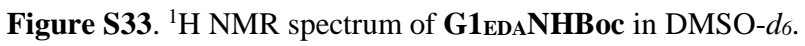

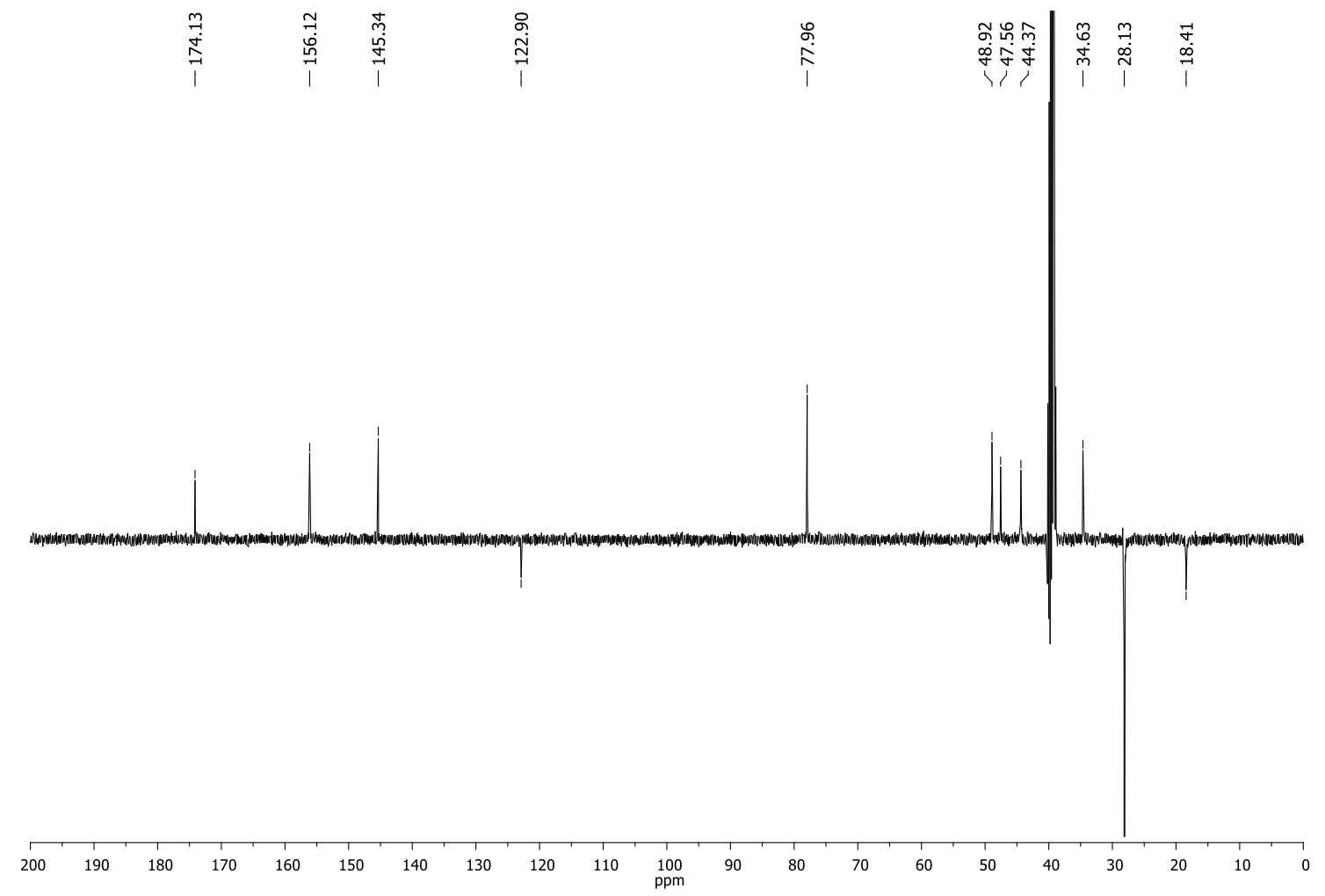

Figure S34. ${ }^{13} \mathrm{C}$ NMR (SEFT) spectrum of G1EDANHBoc in DMSO- $d 6$. 


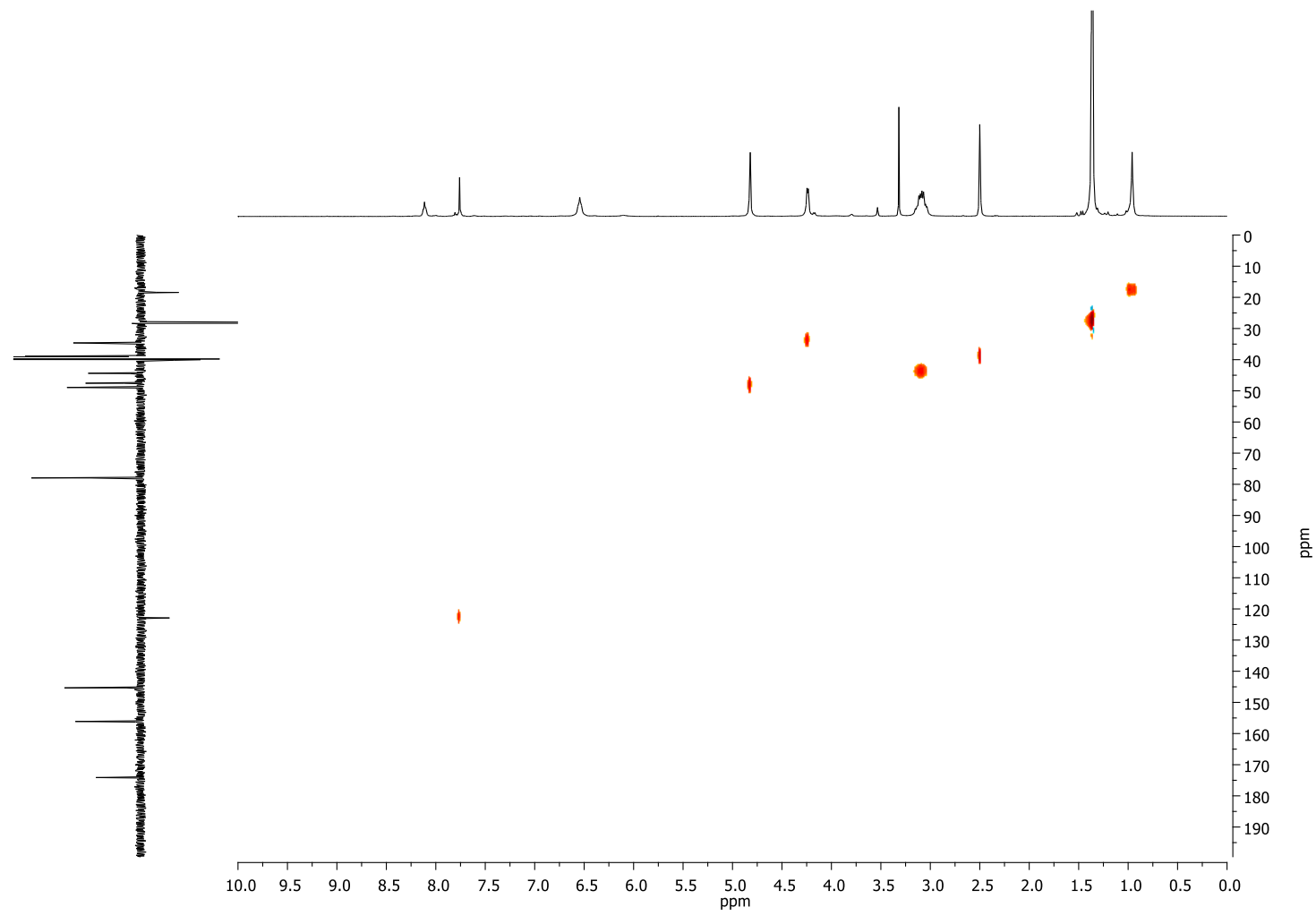

Figure S35. HSQC spectrum of G1EDANHBoc in DMSO- $d_{6}$. 


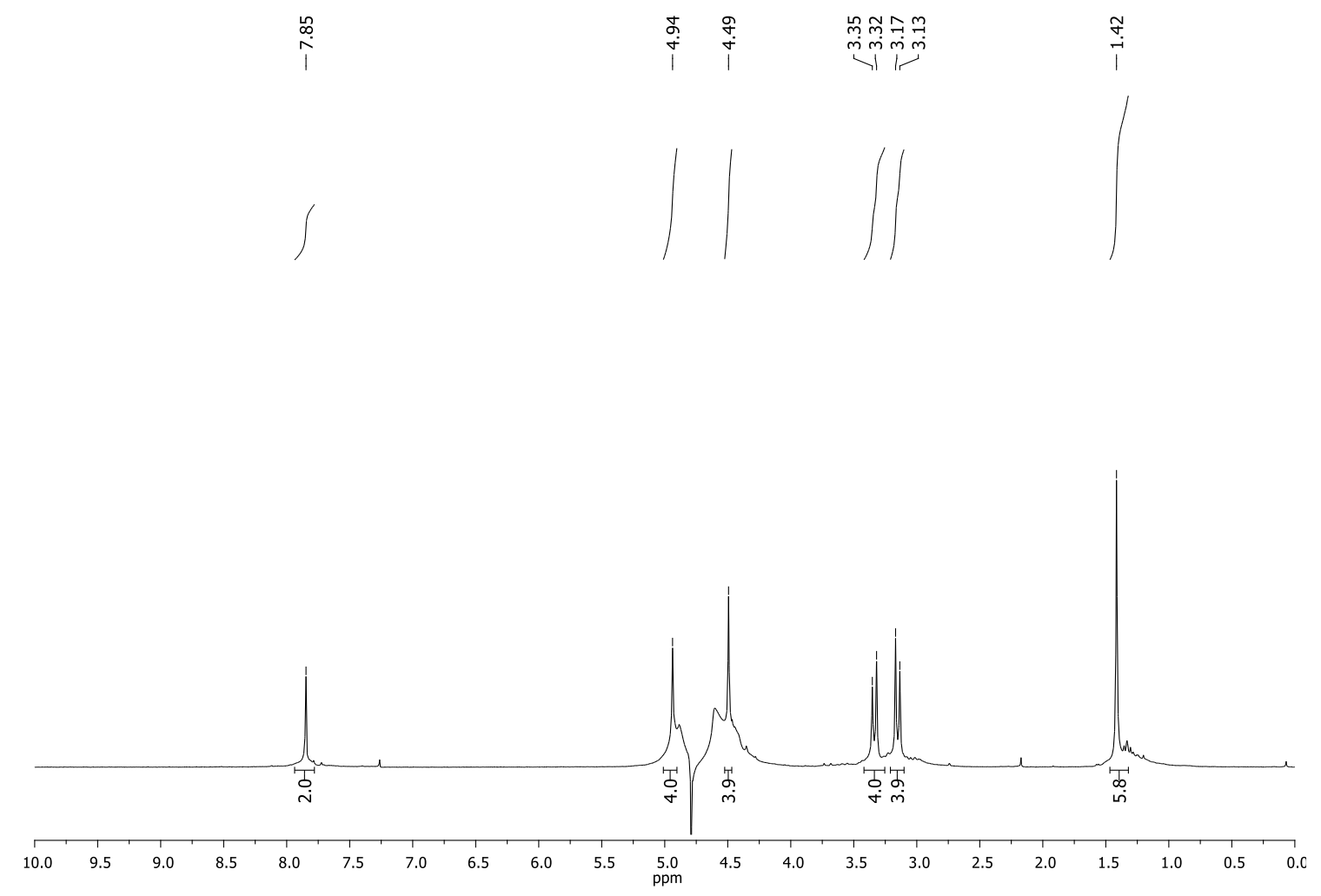

Figure S36. ${ }^{1} \mathrm{H}$ NMR spectrum of $\mathbf{G 1}_{\mathbf{E D A}} \mathbf{N H}_{2}$ in $\mathrm{D}_{2} \mathrm{O}$.

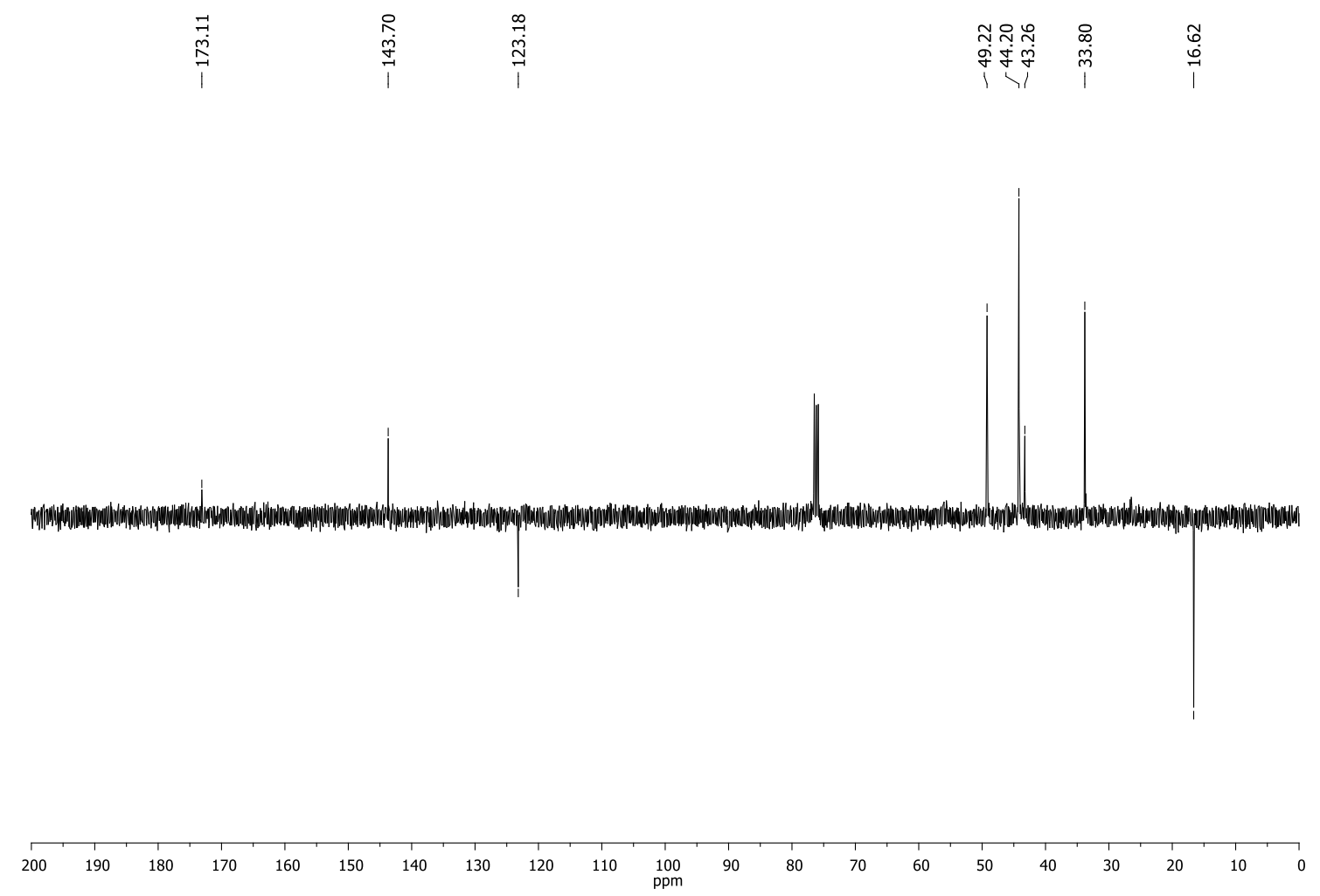

Figure S37. ${ }^{13} \mathrm{C}$ NMR (SEFT) spectrum of $\mathbf{G 1}_{\mathrm{EDA}} \mathbf{N H}_{\mathbf{2}}$ in $\mathrm{D}_{2} \mathrm{O}$. 


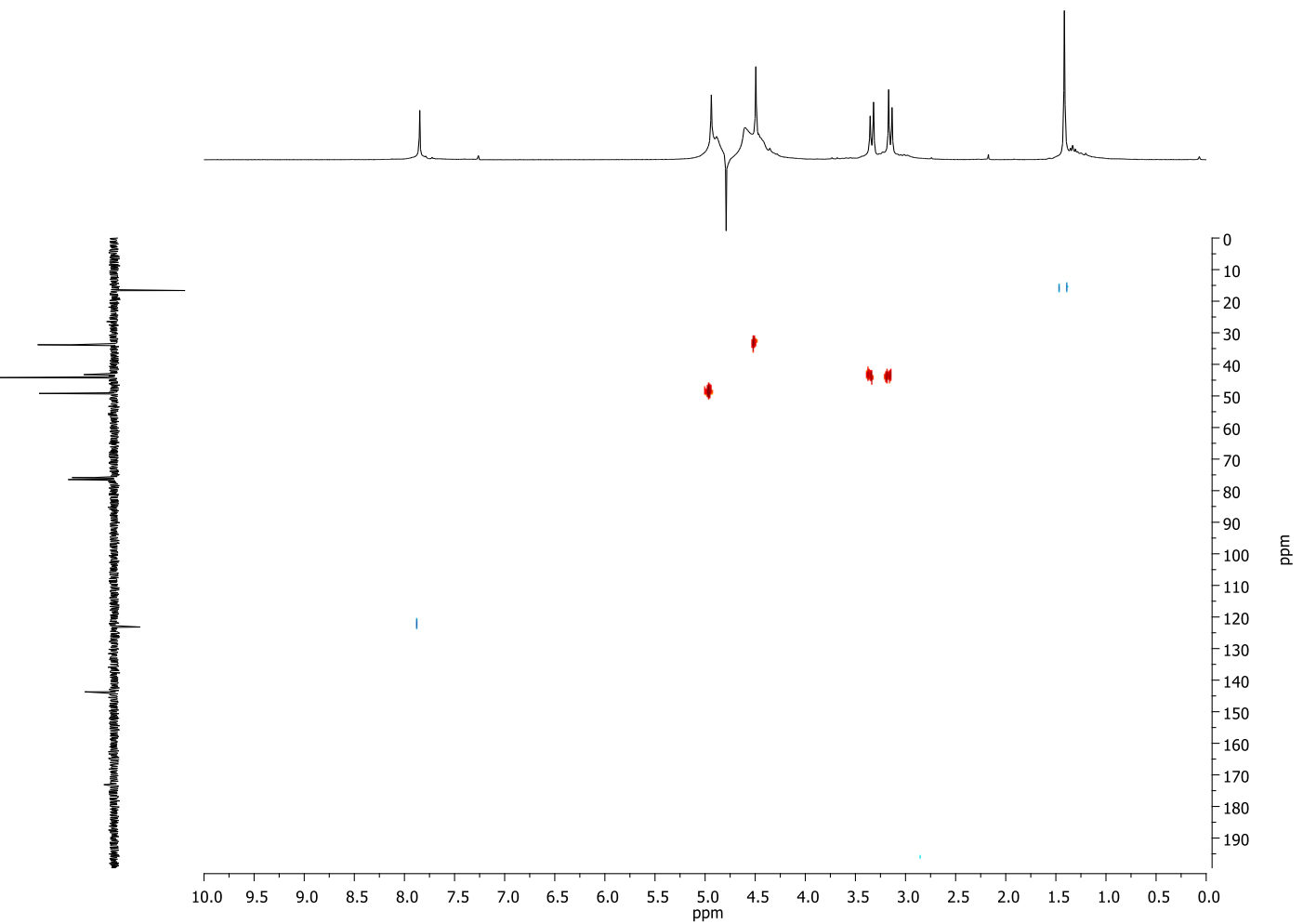

Figure S38. HSQC spectrum of $\mathrm{G}_{\mathrm{EDANH}}$ in $\mathrm{D}_{2} \mathrm{O}$. 


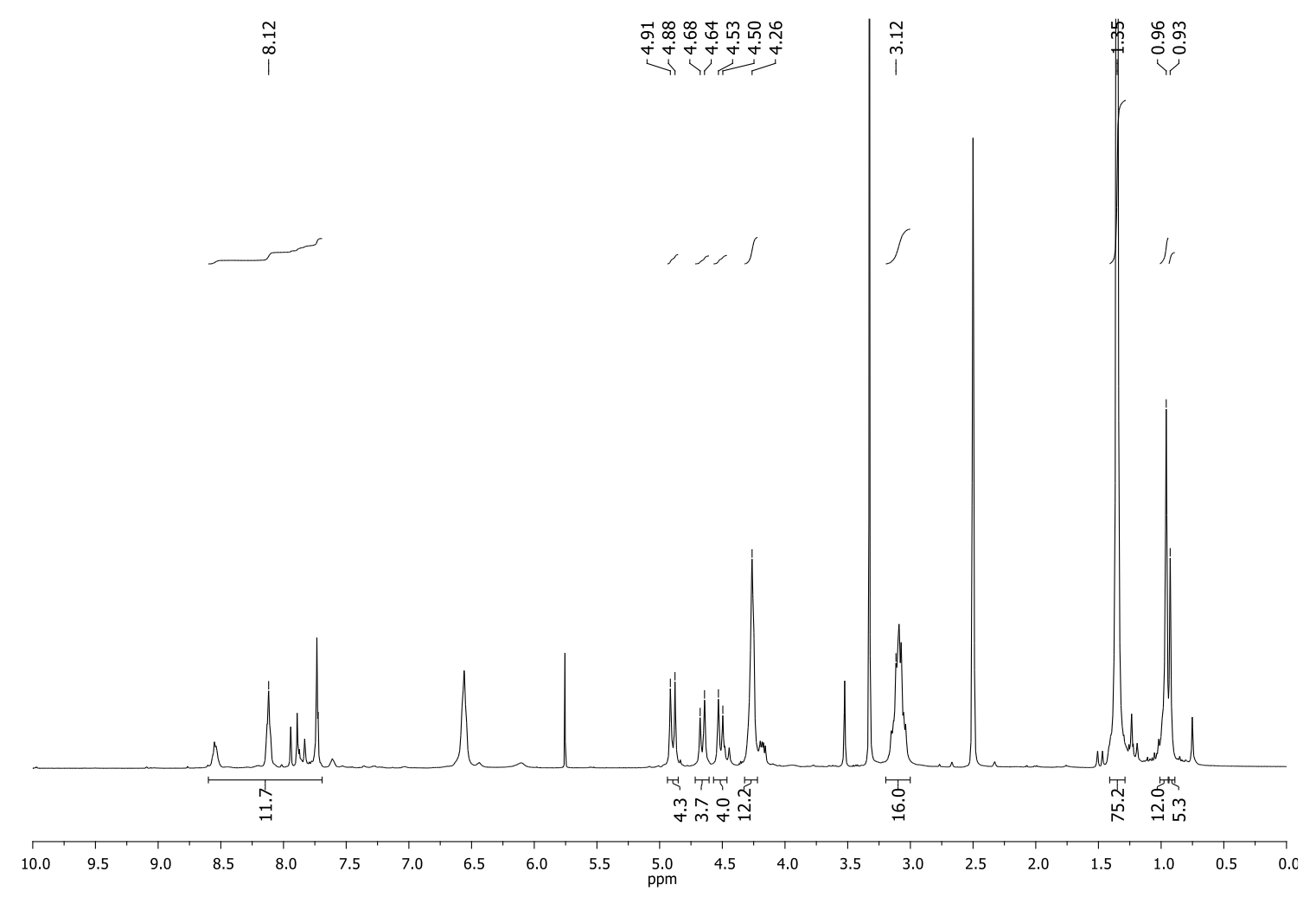

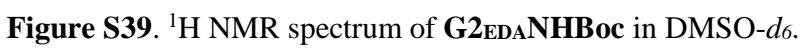

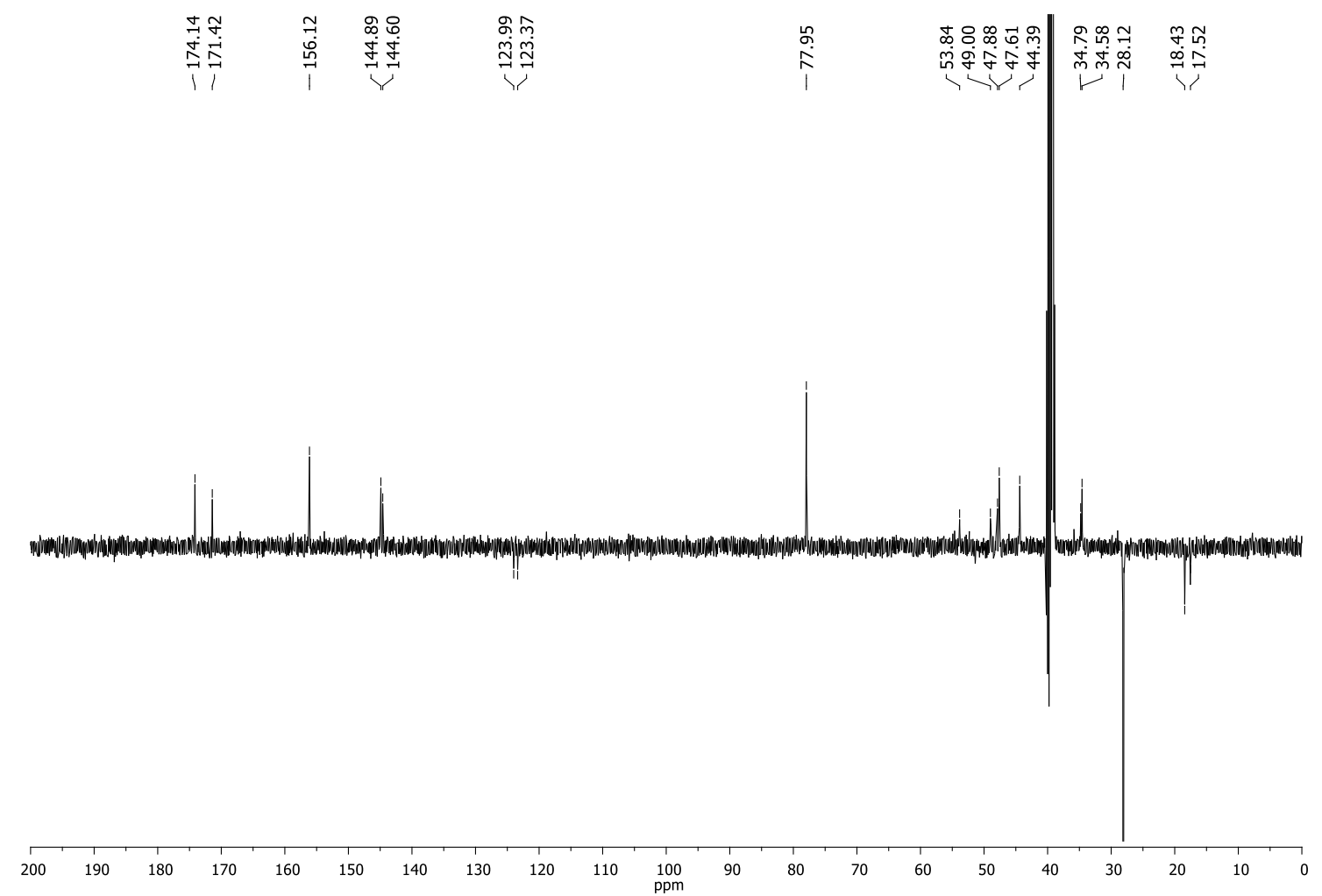

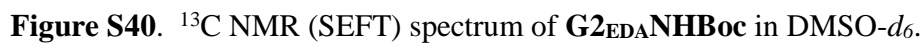




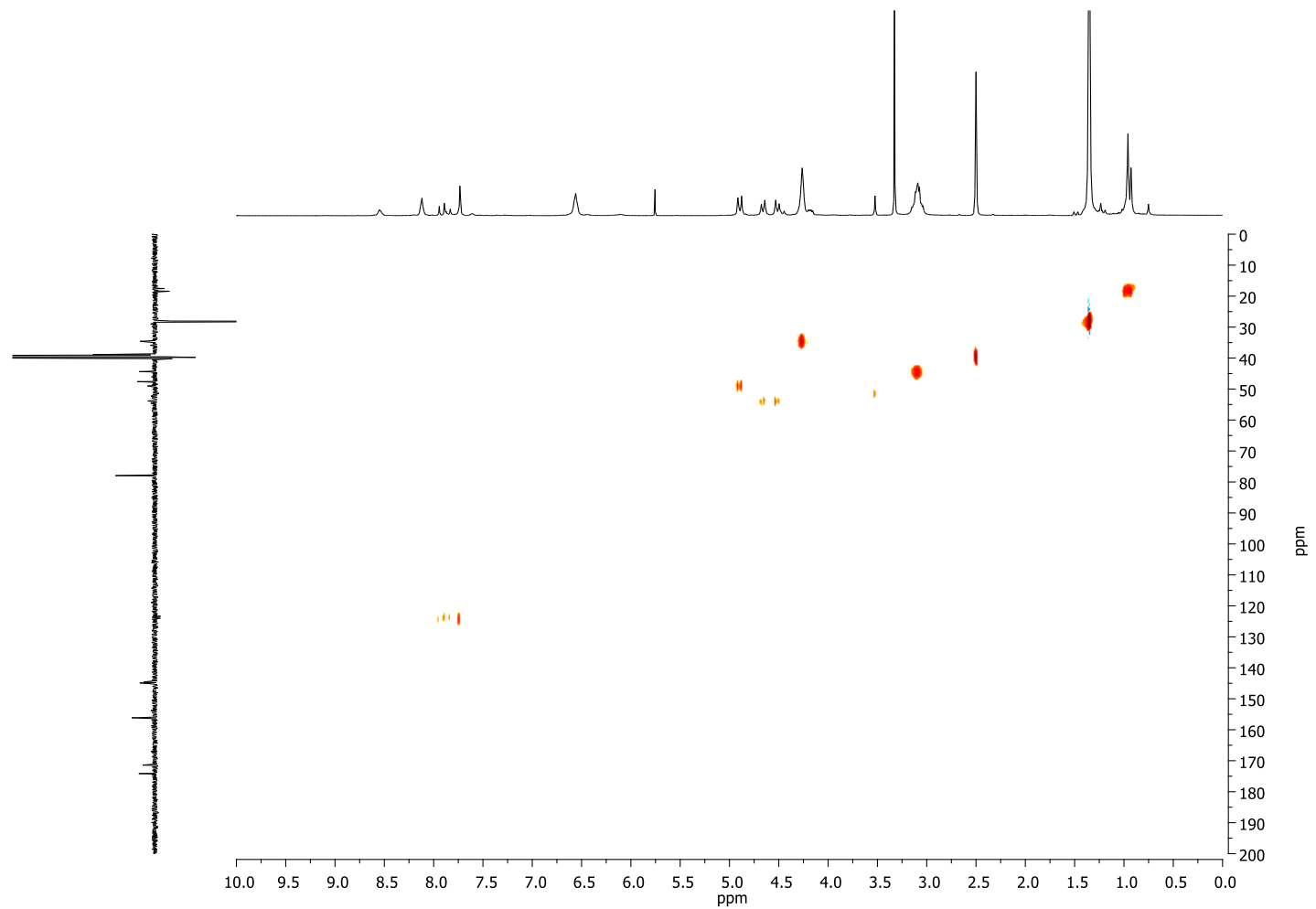

Figure S41. HSQC spectrum of G2EDANHBoc in DMSO- $d_{6}$. 


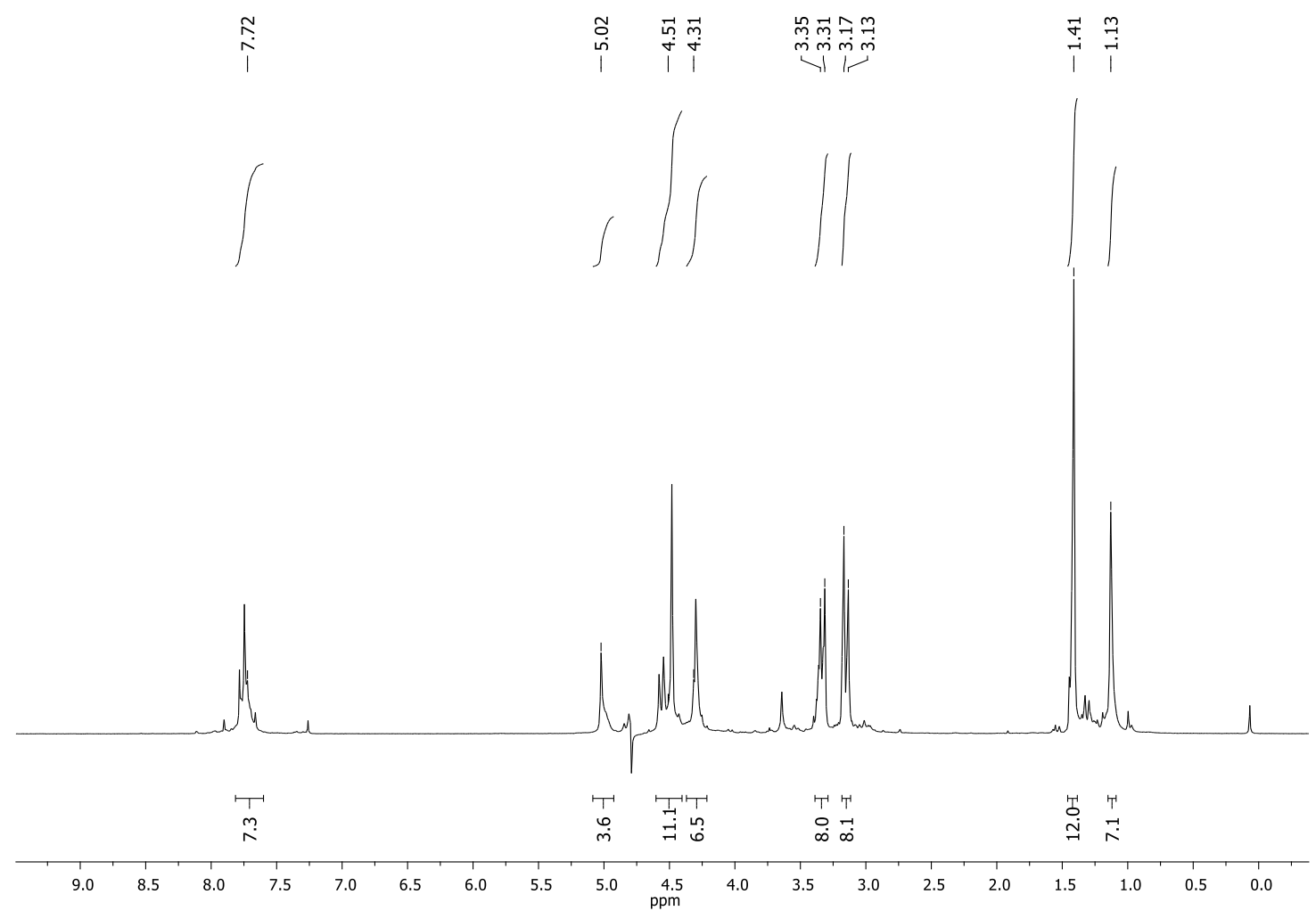

Figure S42. ${ }^{1} \mathrm{H}$ NMR spectrum of $\mathbf{G} \mathbf{2}_{\mathbf{E D A}} \mathbf{N H}_{2}$ in $\mathrm{D}_{2} \mathrm{O}$.

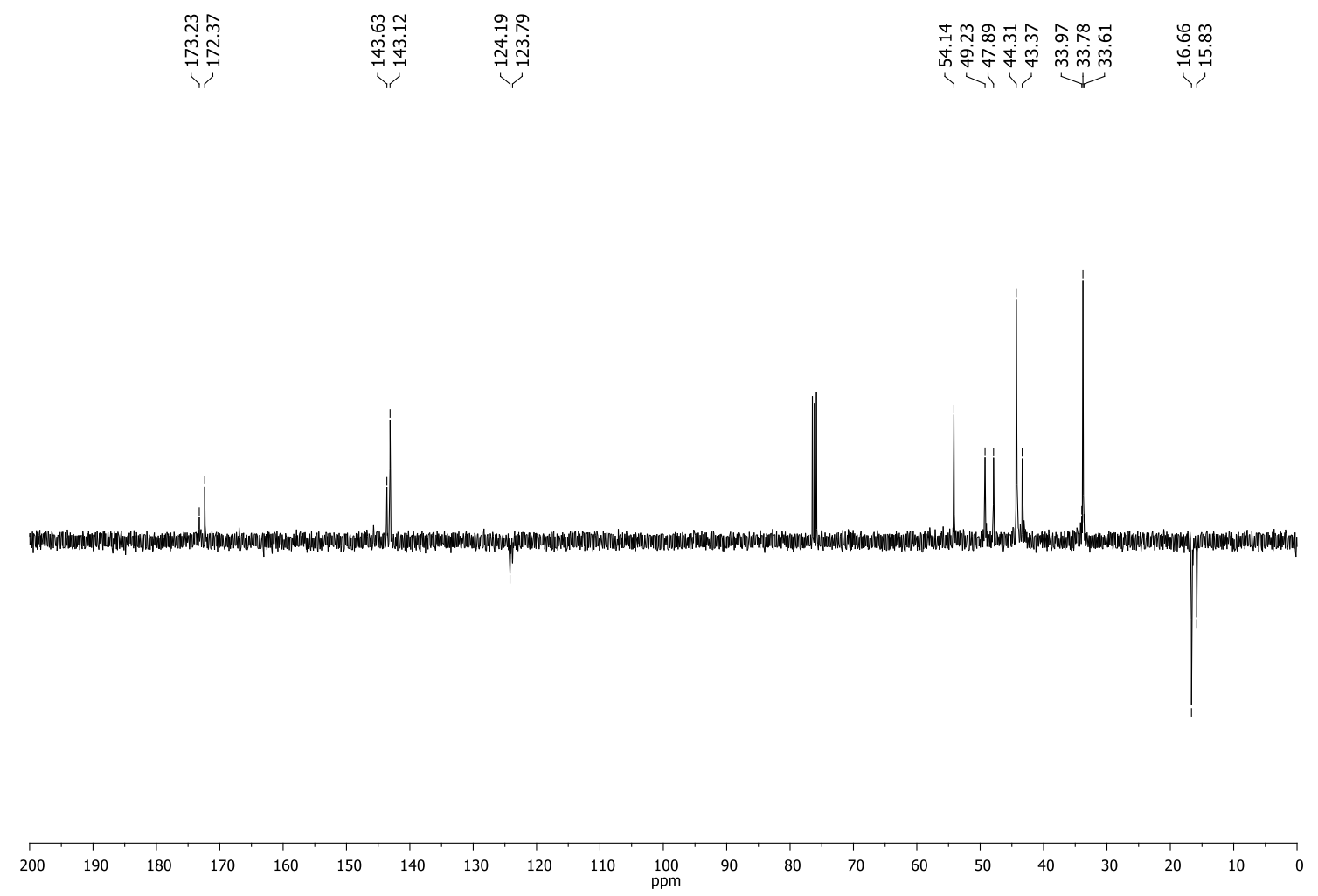

Figure S43. ${ }^{13} \mathrm{C}$ NMR (SEFT) spectrum of $\mathbf{G 2} \mathbf{E D A}_{\mathbf{E A}} \mathbf{N H}_{\mathbf{2}}$ in $\mathrm{D}_{2} \mathrm{O}$. 


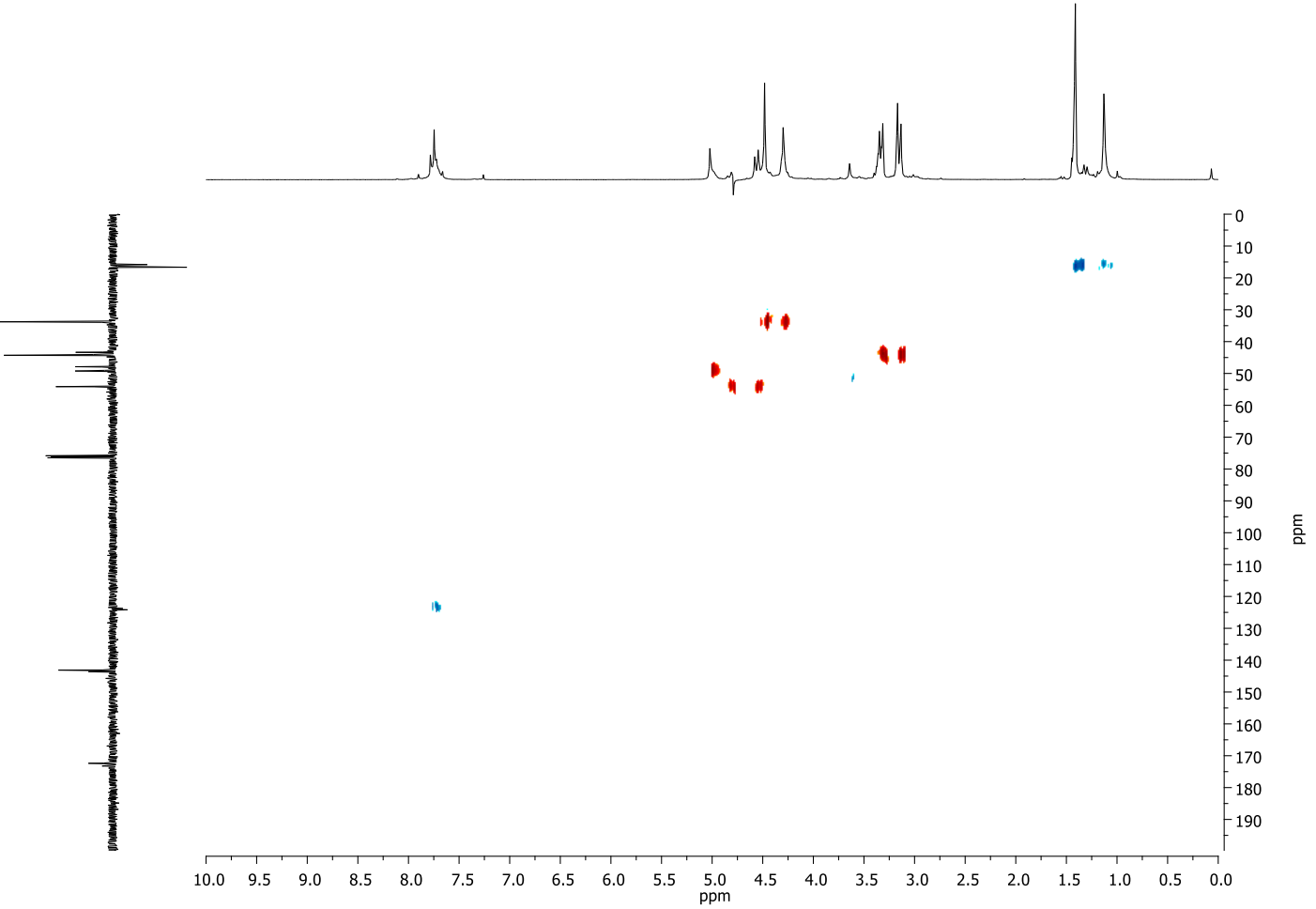

Figure S44. HSQC spectrum of $\mathrm{G} 2 \mathrm{EDANH}_{2}$ in $\mathrm{D}_{2} \mathrm{O}$. 


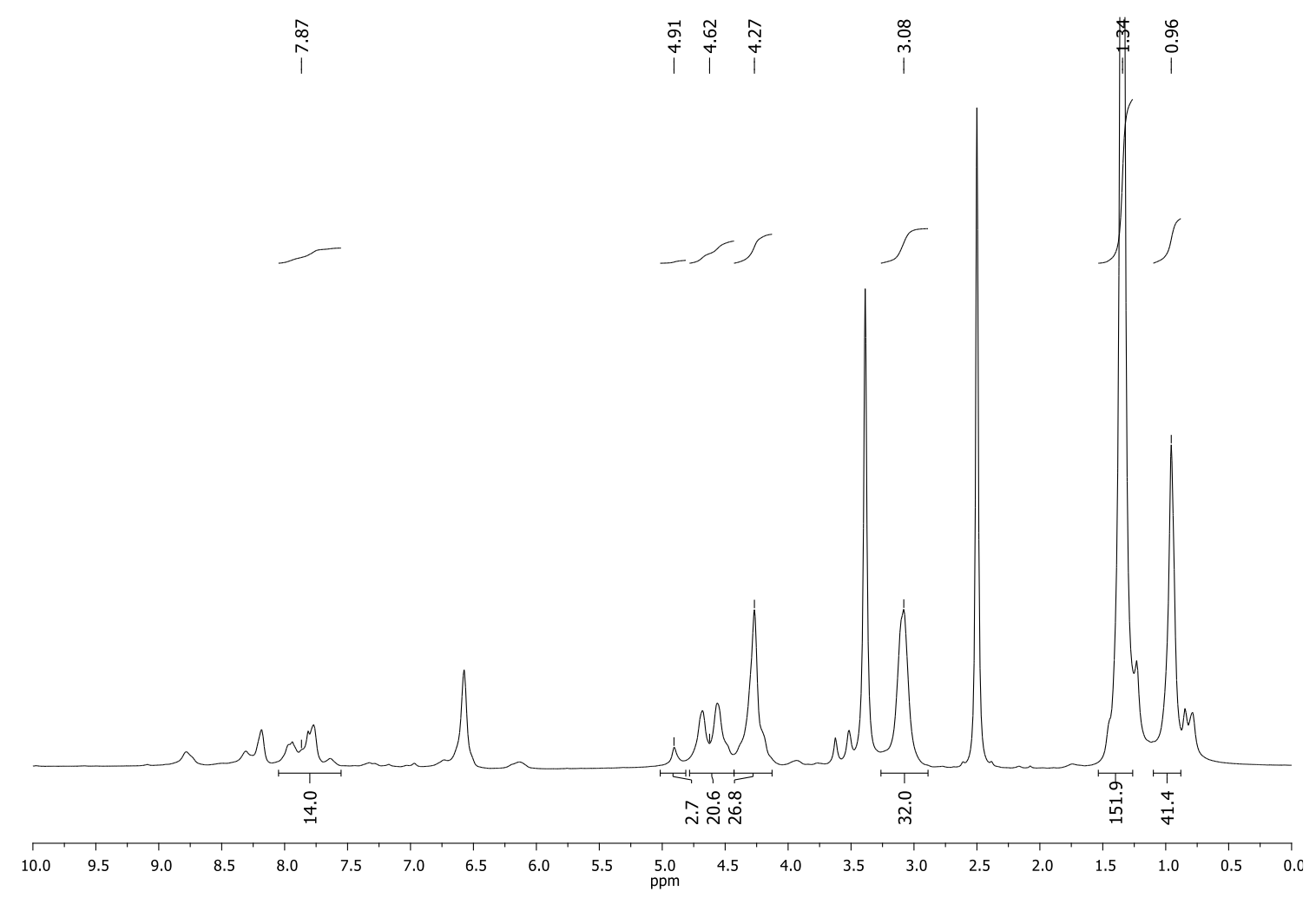

Figure S45. ${ }^{1} \mathrm{H}$ NMR spectrum of $\mathbf{G} 3_{\text {EDANHBoc in DMSO- }} d_{6}$.

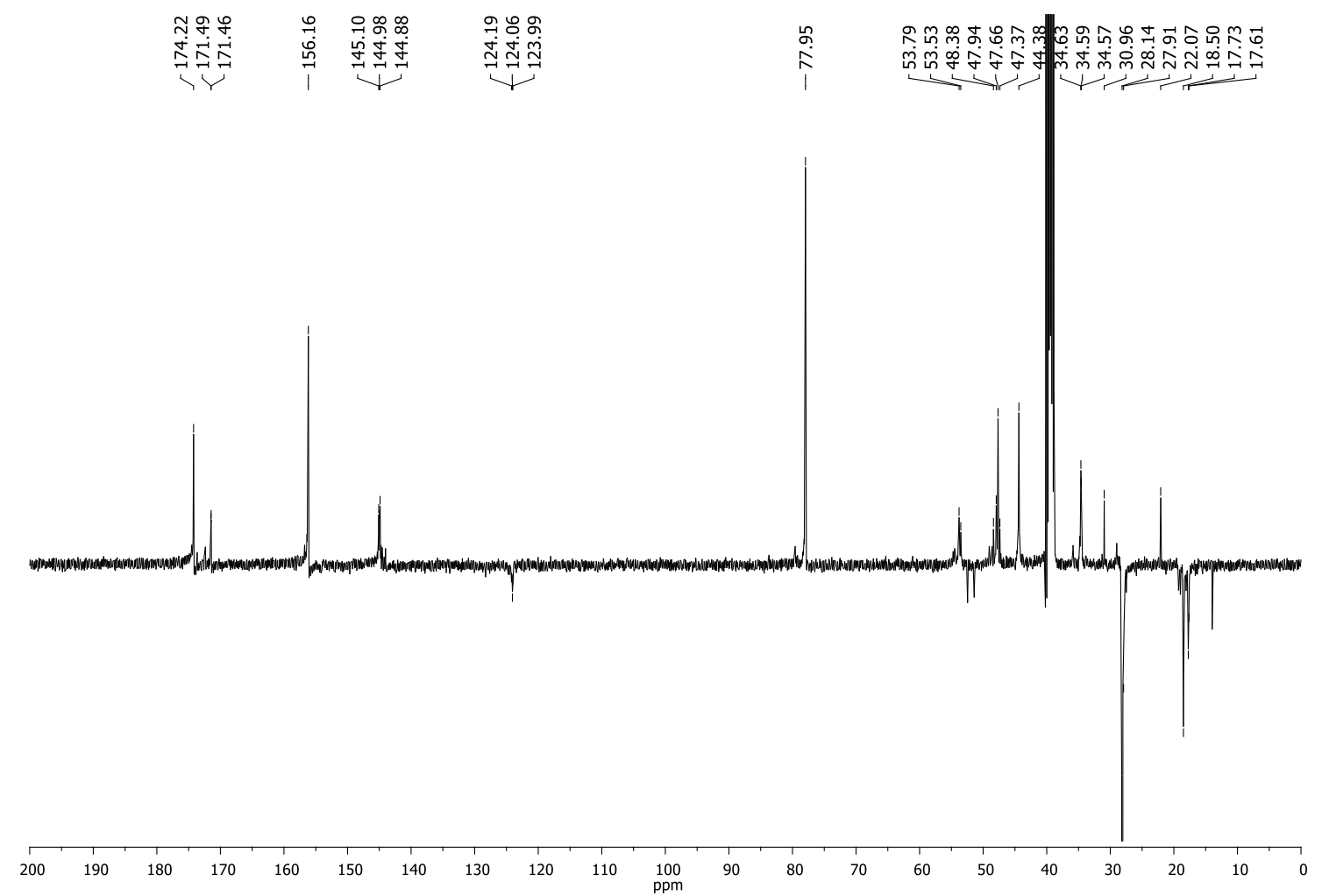

Figure S46. ${ }^{13} \mathrm{C}$ NMR (SEFT) spectrum of G3EDANHBoc in DMSO- $d_{6}$. 


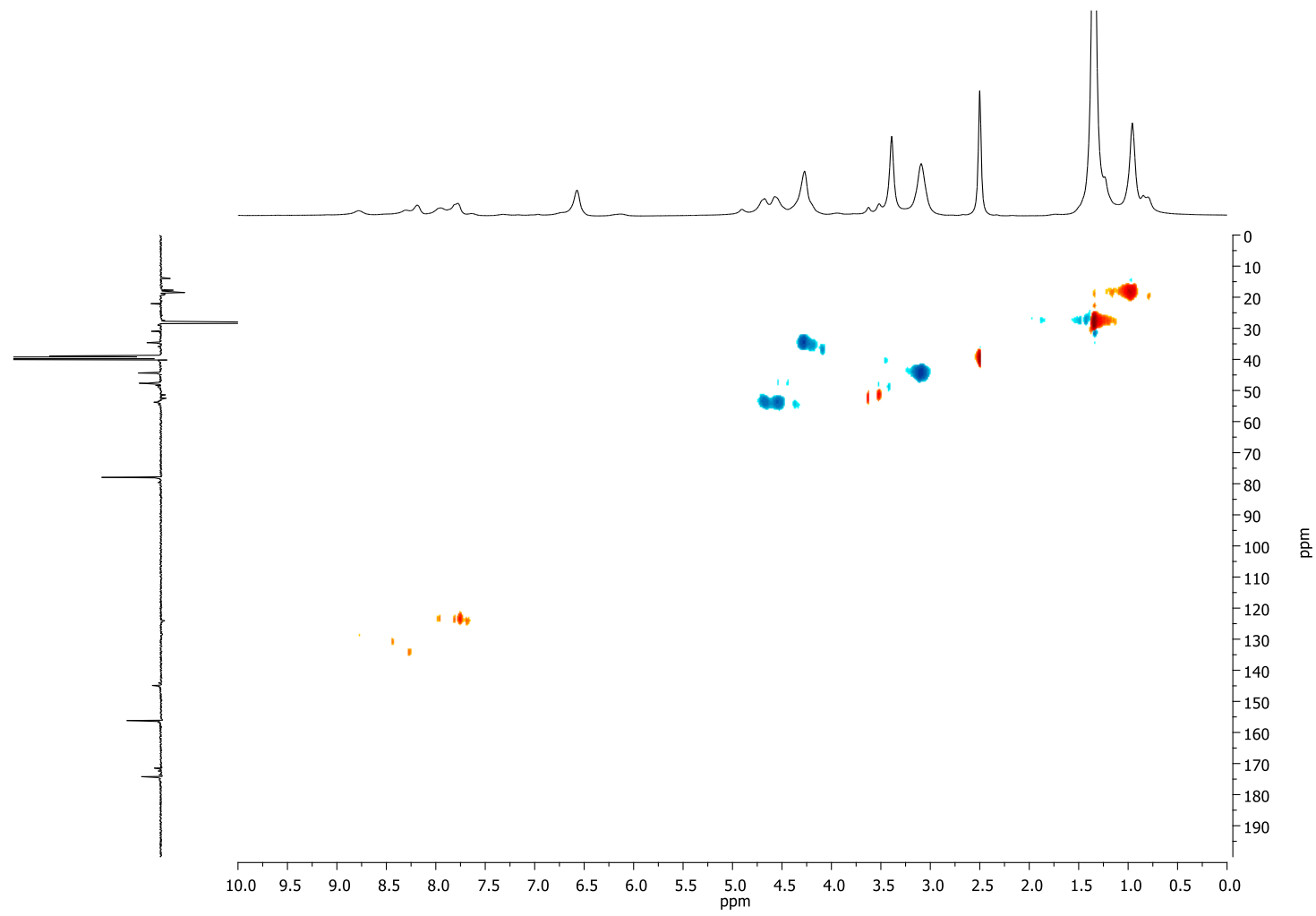

Figure S47. HSQC spectrum of G3EDANHBoc in DMSO- $d 6$. 


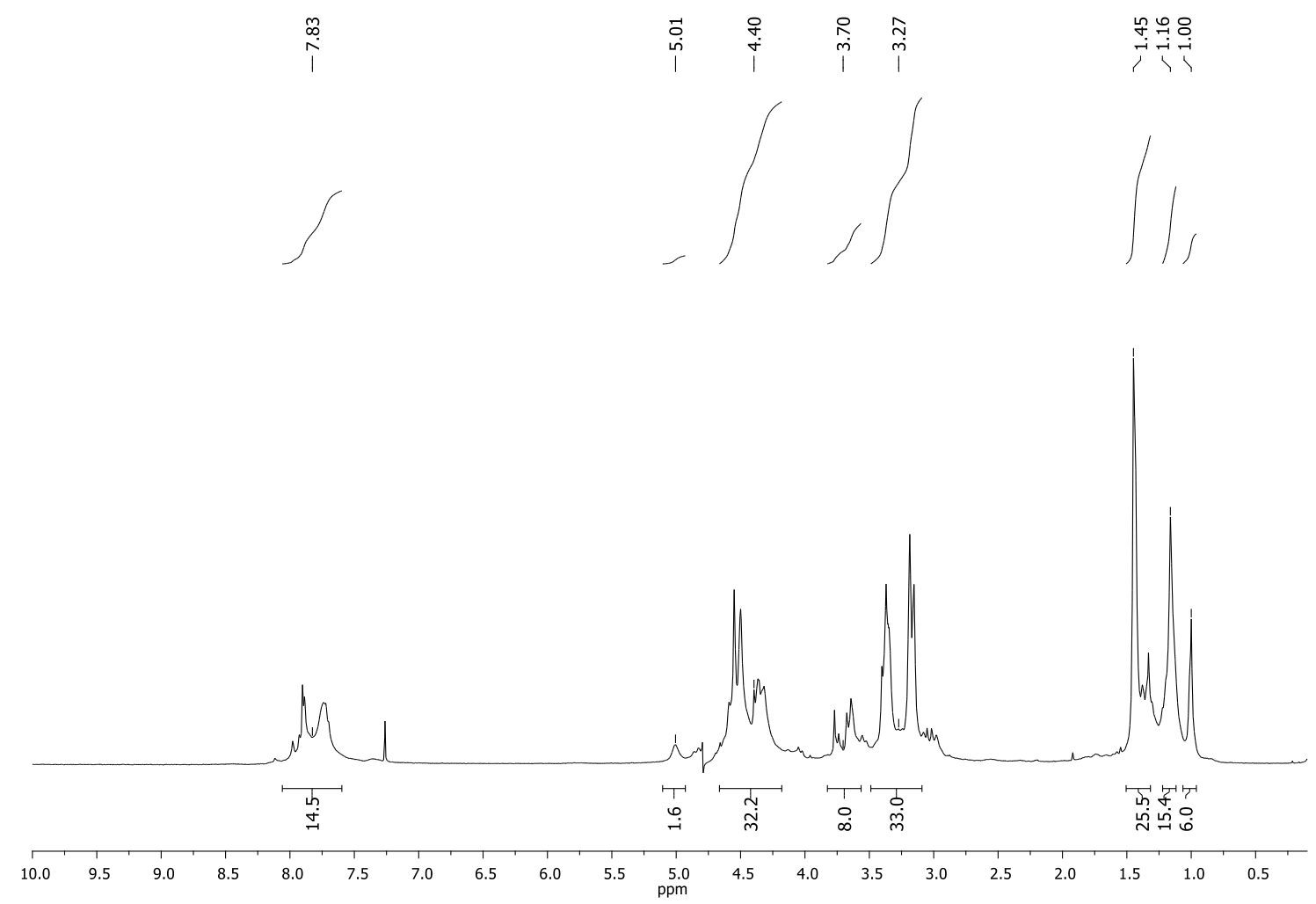

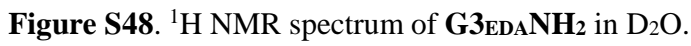

\begin{tabular}{|c|c|c|c|}
\hline 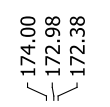 & 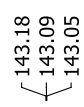 & 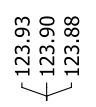 & 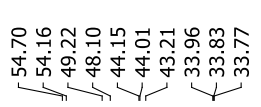 \\
\hline
\end{tabular}

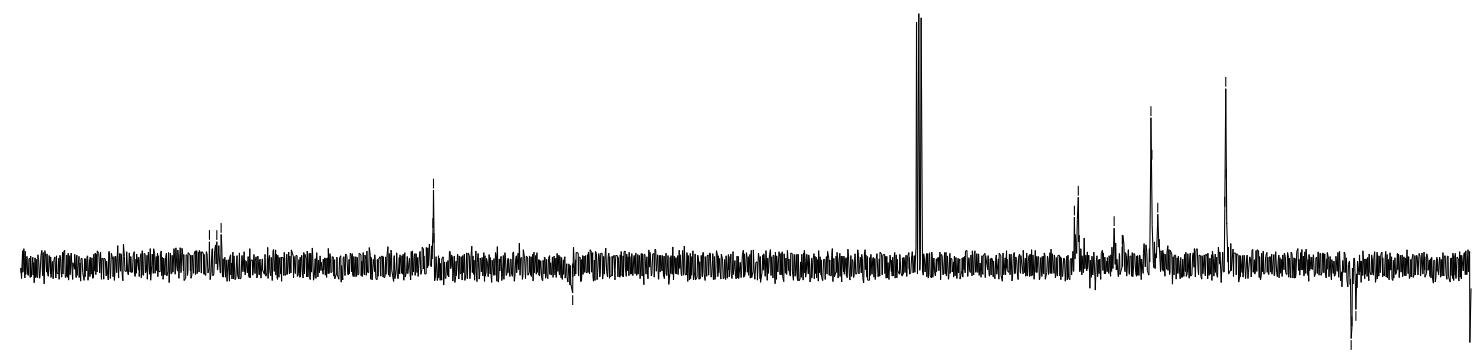

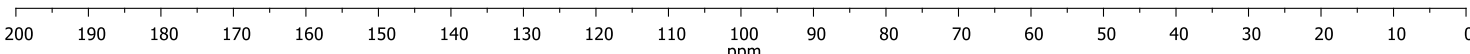

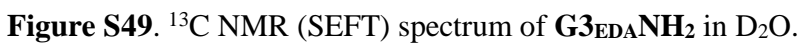




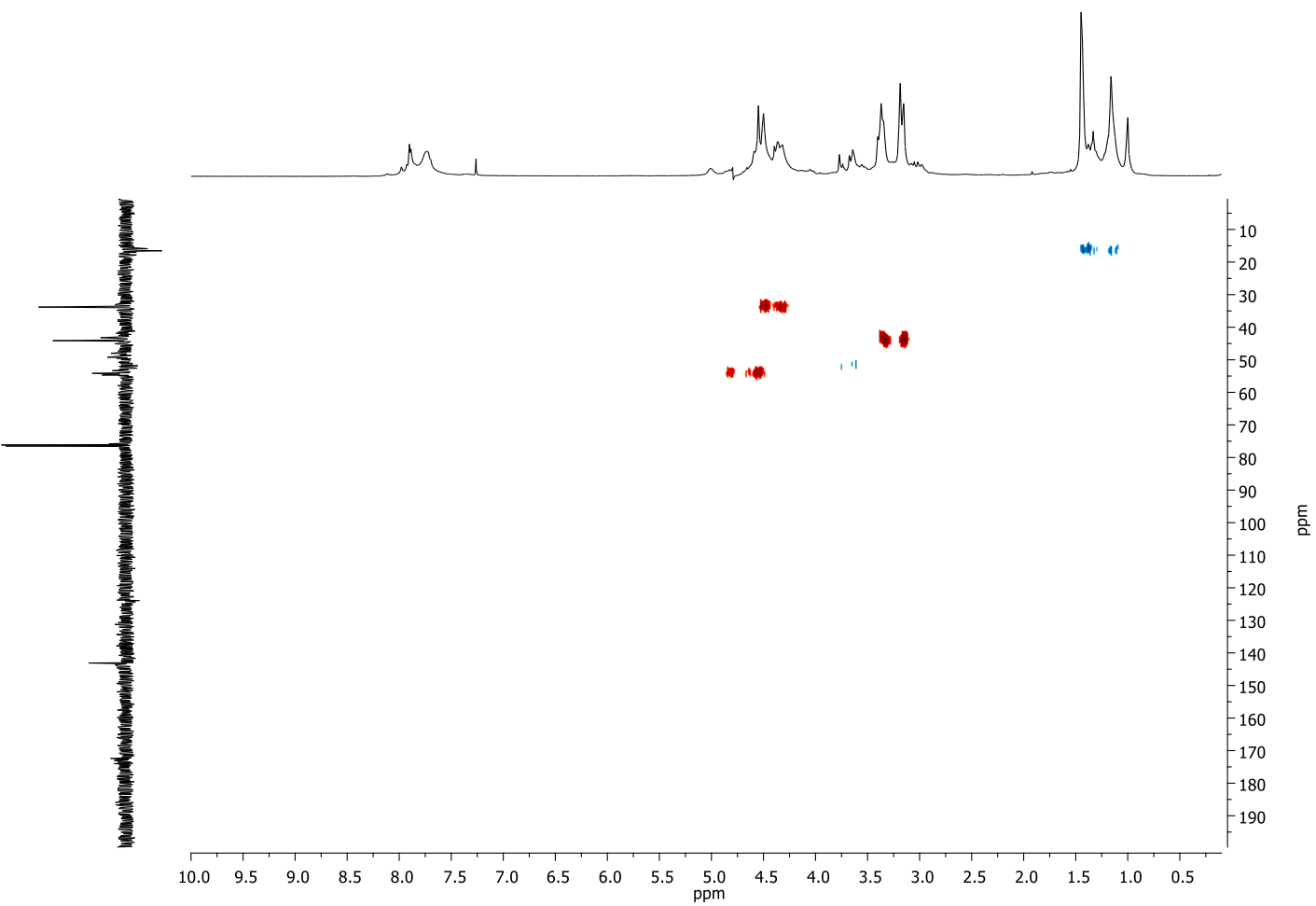

Figure S50. HSQC spectrum of $\mathrm{G}_{3} \mathrm{EDANH}_{2}$ in $\mathrm{D}_{2} \mathrm{O}$. 

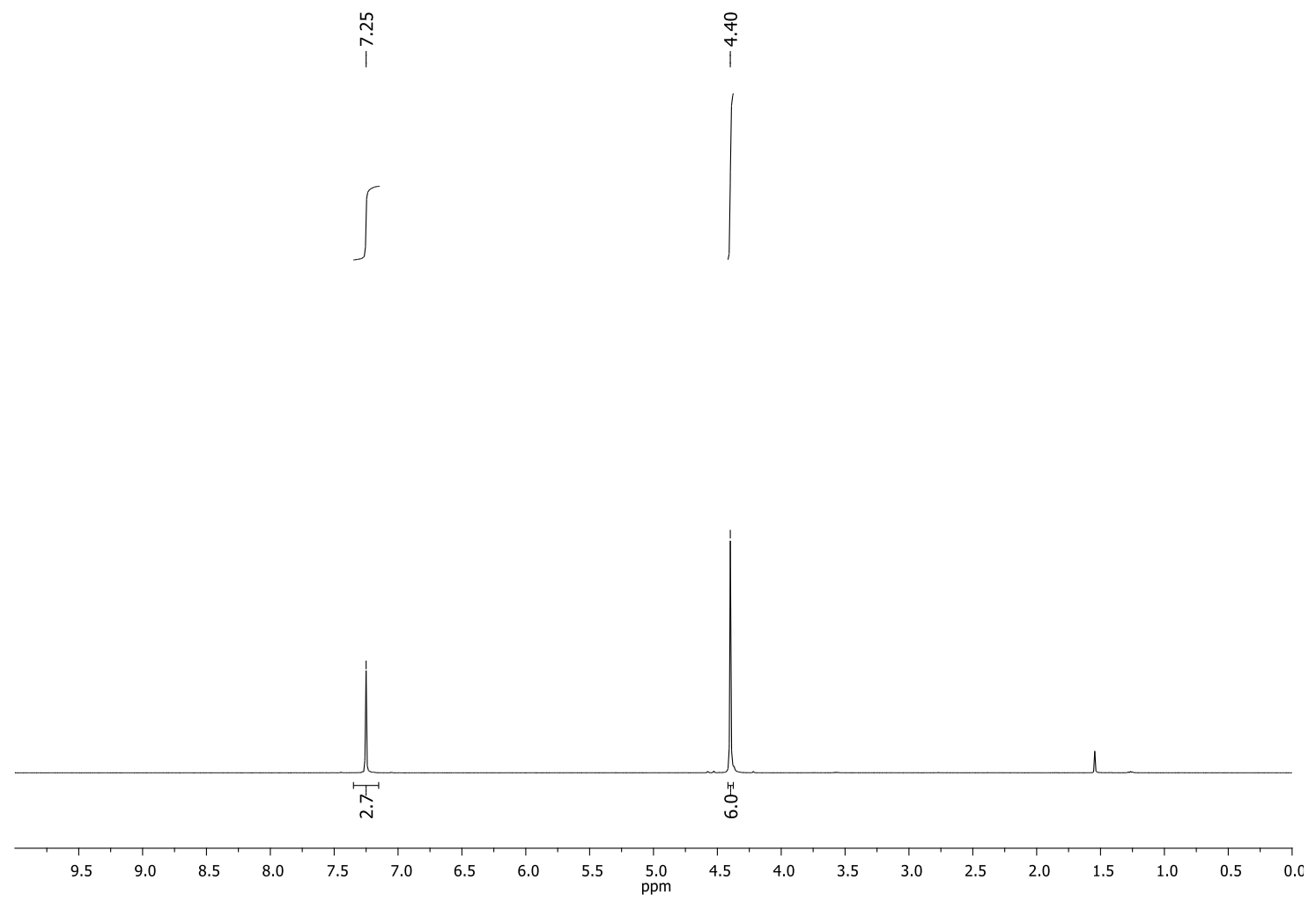

Figure S51. ${ }^{1} \mathrm{H}$ NMR spectrum of 1,3,5-tris(azidomethylene)benzene in $\mathrm{CDCl}_{3}$. 


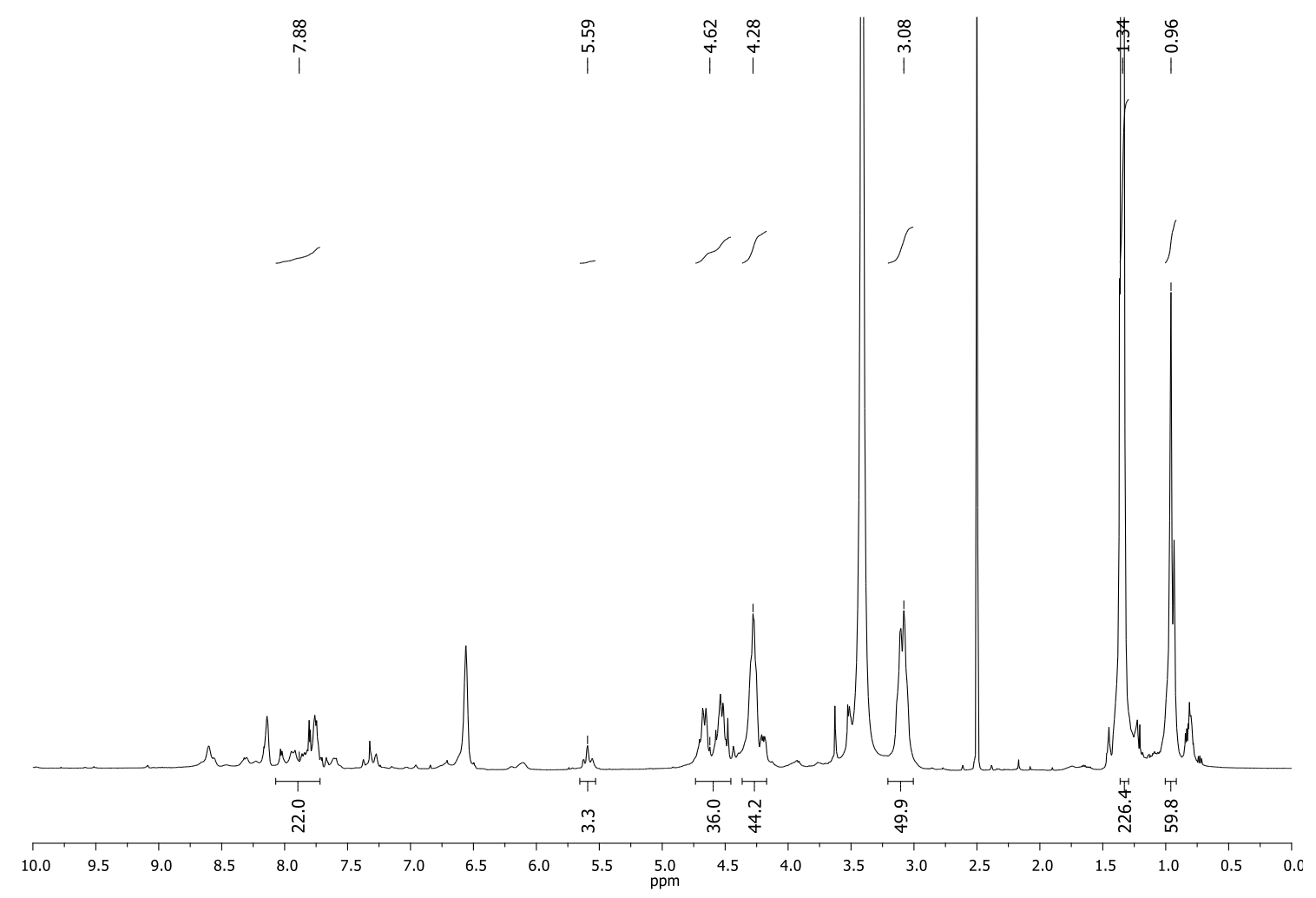

Figure S52. ${ }^{1} \mathrm{H}$ NMR spectrum of $\mathbf{G 3}_{3 \mathbf{3 B}} \mathbf{N H B o c}$ in DMSO- $d_{6}$.

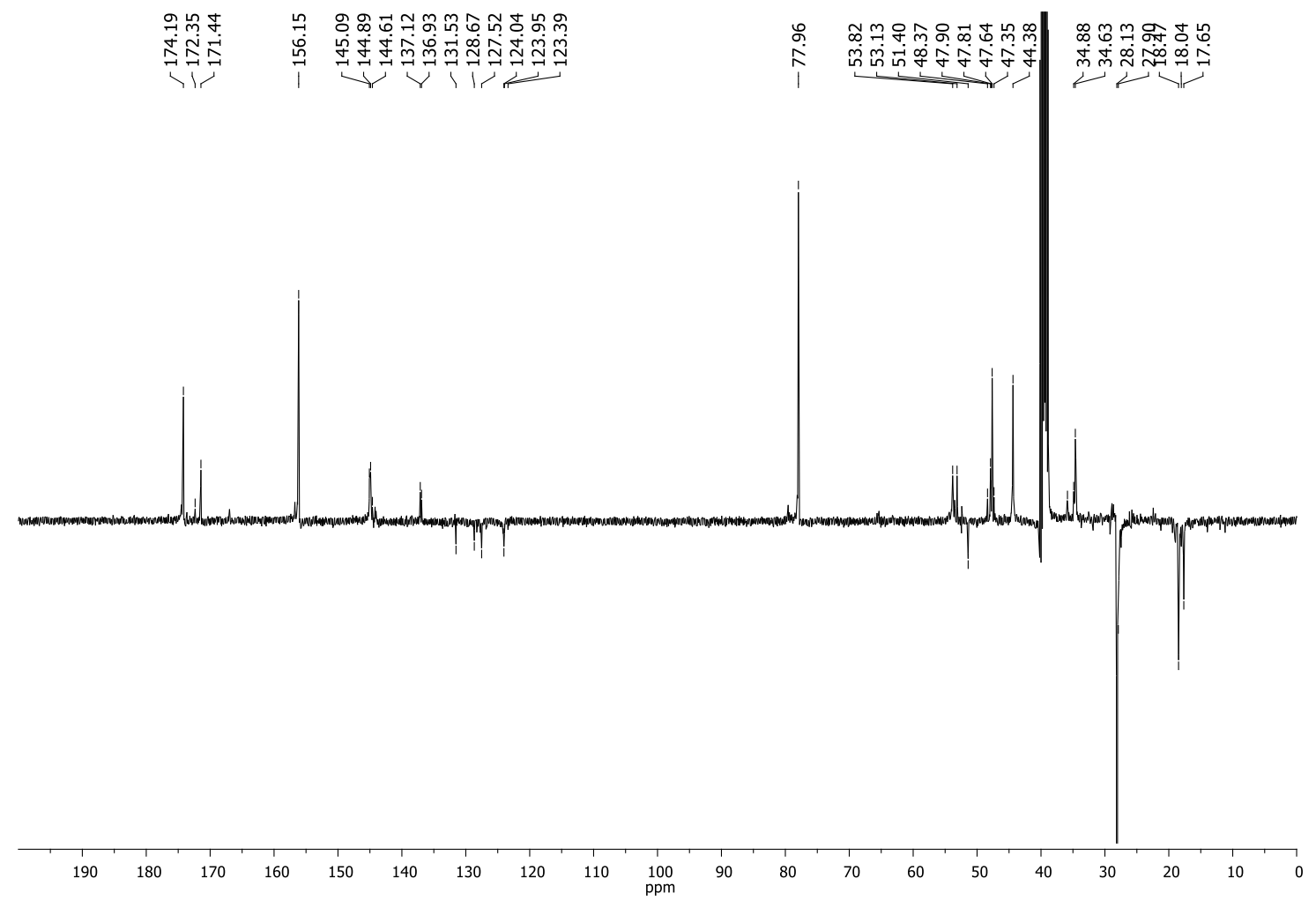

Figure S53. ${ }^{13} \mathrm{C}$ NMR (SEFT) spectrum of $\mathbf{G 3} 3_{3 \mathrm{AB}}$ NHBoc in DMSO- $d_{6}$. 


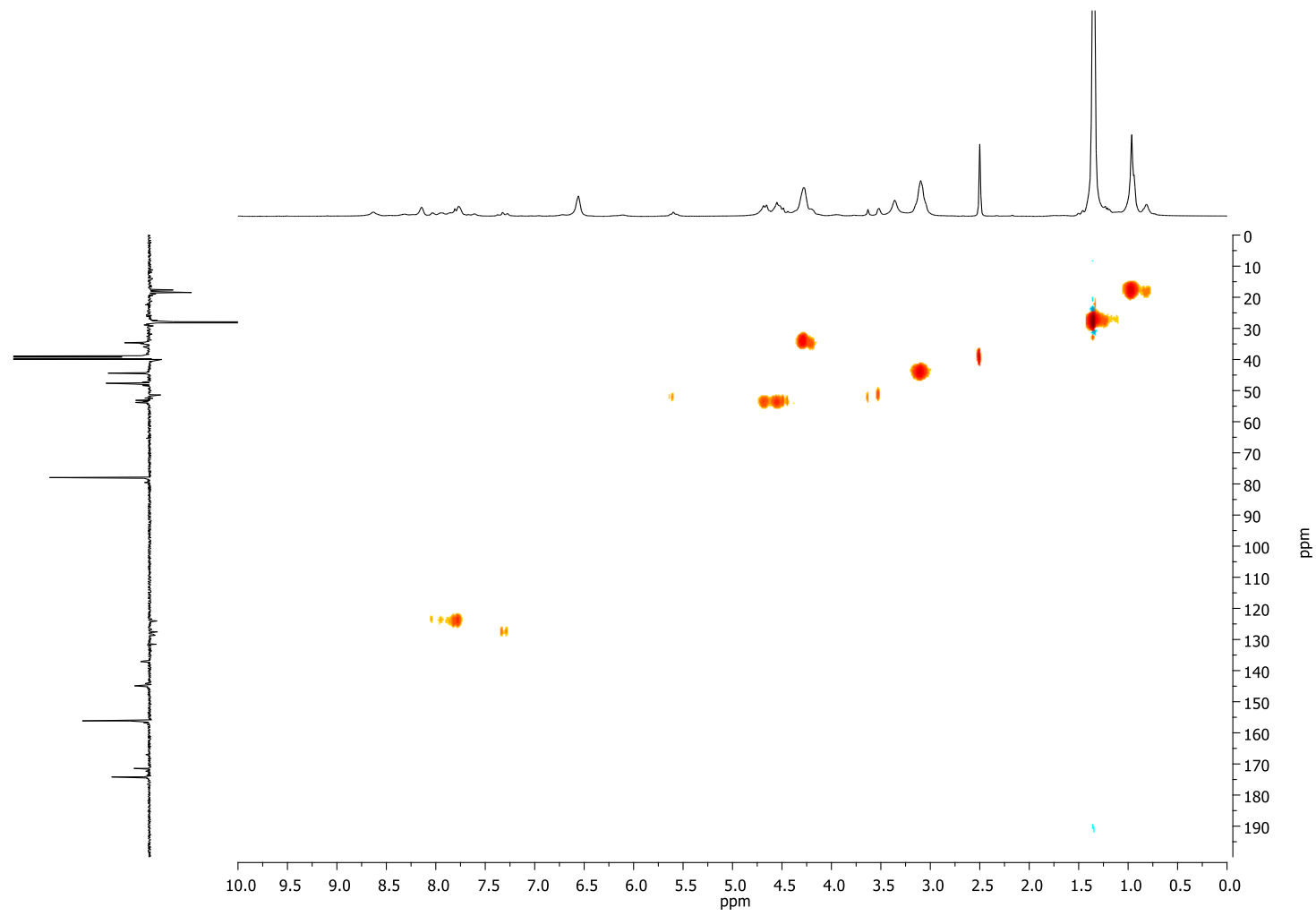

Figure S54. HSQC spectrum of G33 ${ }_{3 \mathrm{BB}} \mathrm{NHBoc}$ in DMSO-d6. 


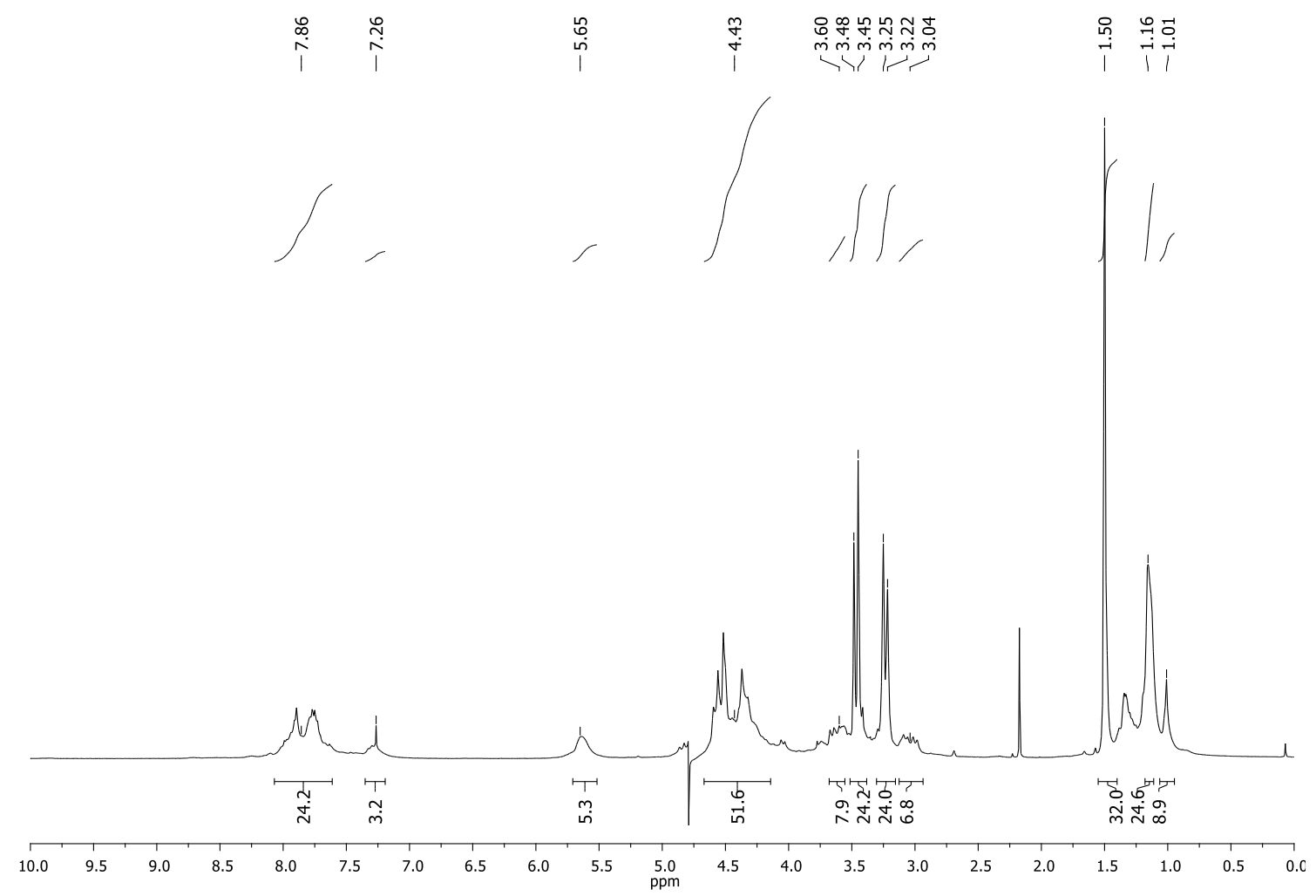

Figure S55. ${ }^{1} \mathrm{H}$ NMR spectrum of $\mathbf{G 3}_{3} \mathbf{A B}_{\mathbf{B}} \mathbf{N H}_{2}$ in $\mathrm{D}_{2} \mathrm{O}$.

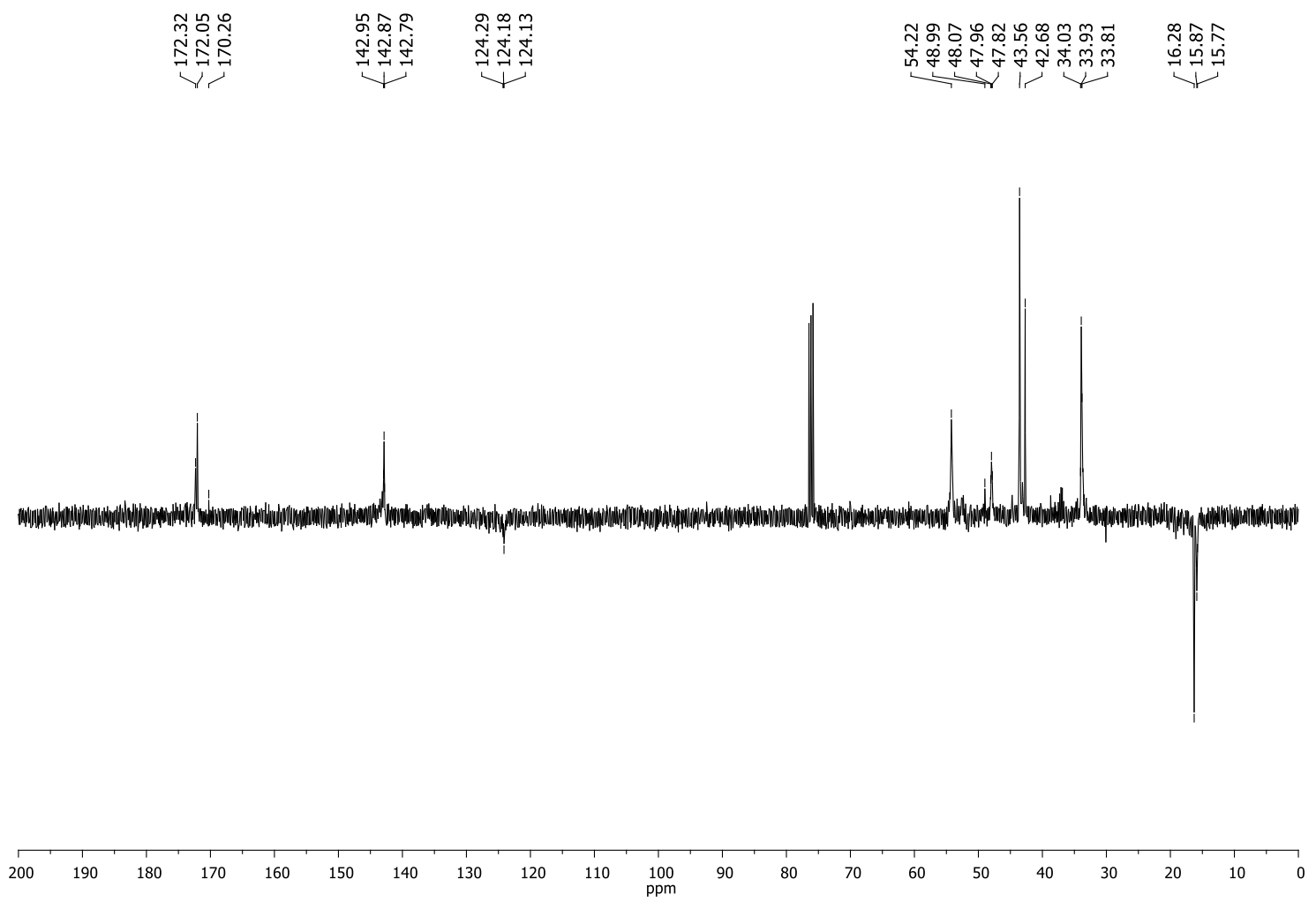

Figure S56. ${ }^{13} \mathrm{C}$ NMR (SEFT) spectrum of $\mathbf{G 3}_{3} \mathbf{A B}_{\mathbf{B B}} \mathbf{N H}_{\mathbf{2}}$ in $\mathrm{D}_{2} \mathrm{O}$. 


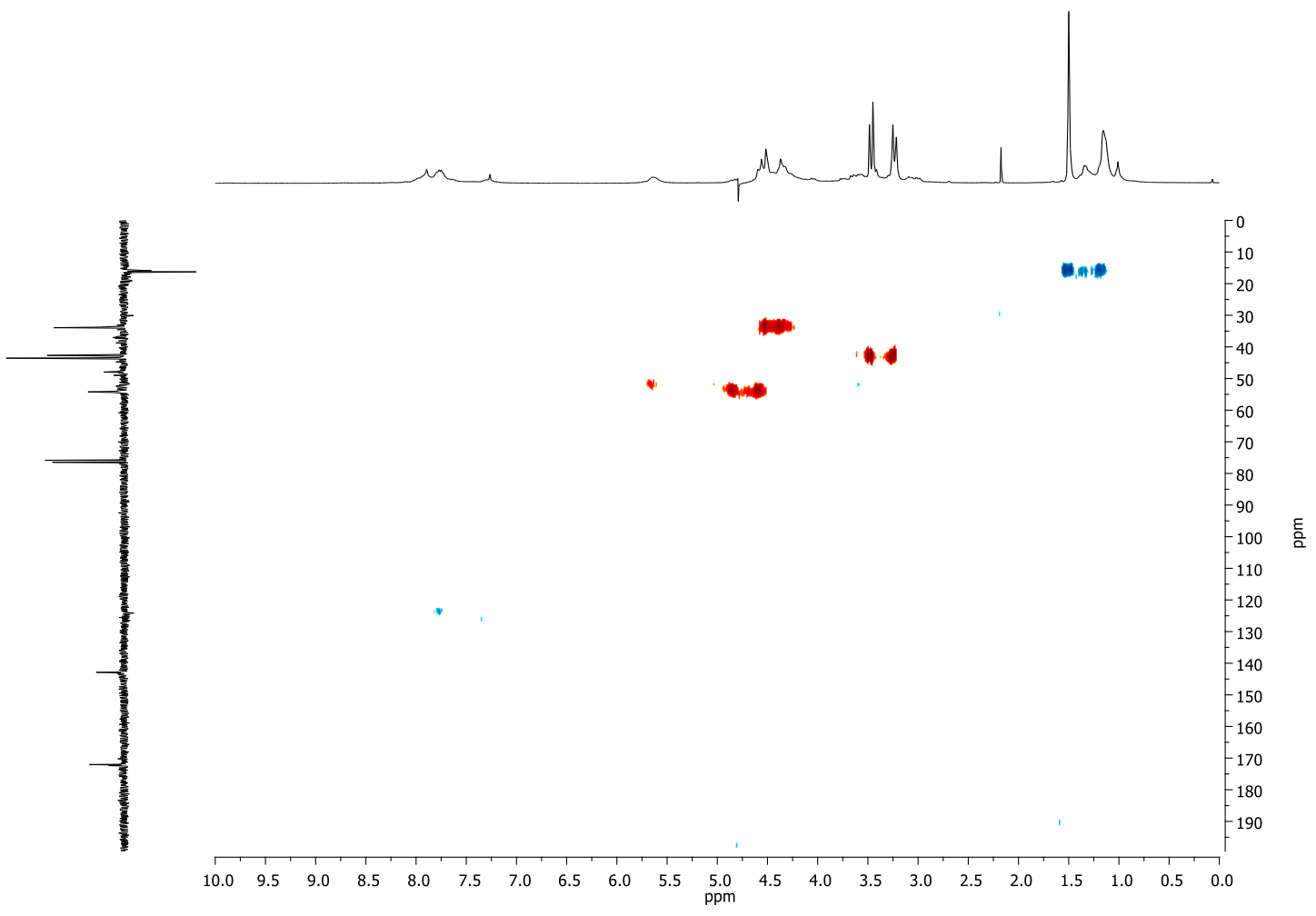

Figure S57. HSQC spectrum of $\mathrm{G}_{3}{ }_{3 \mathrm{ABNH}} \mathrm{N}_{2}$ in $\mathrm{D}_{2} \mathrm{O}$.

S-38 


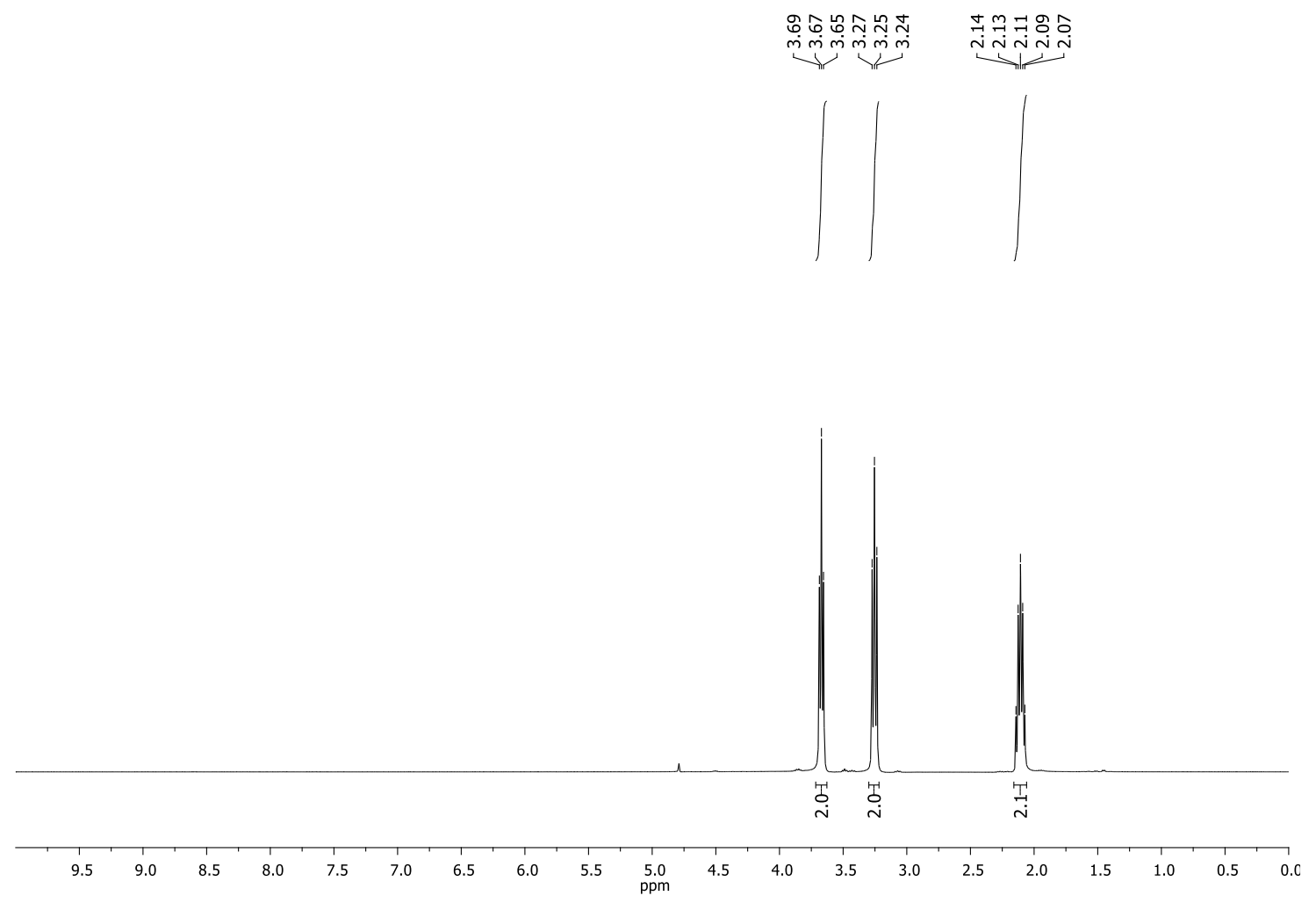

Figure S58. ${ }^{1} \mathrm{H}$ NMR spectrum of 3 -azidopropylamine in $\mathrm{D}_{2} \mathrm{O}$. 

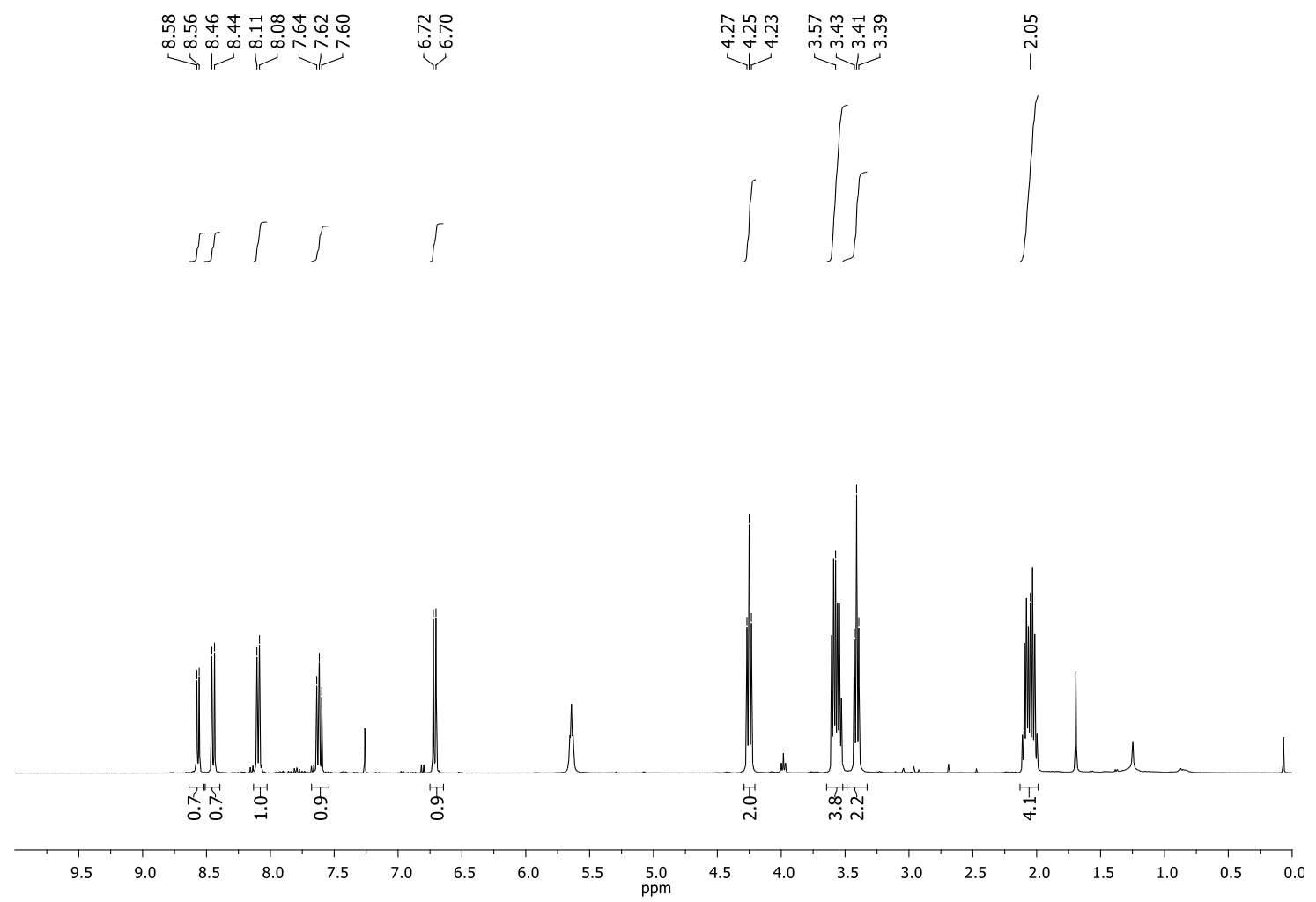

Figure S59. ${ }^{1} \mathrm{H}$ NMR spectrum of $N$-(2-azidopropyl)-4-((2-azidopropyl)amino)-1,8-naphtalimide inCDCl ${ }_{3}$.

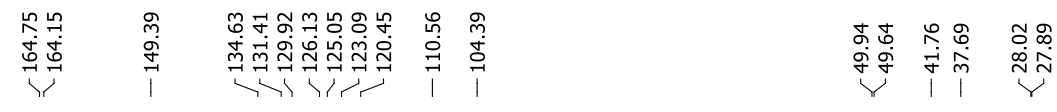

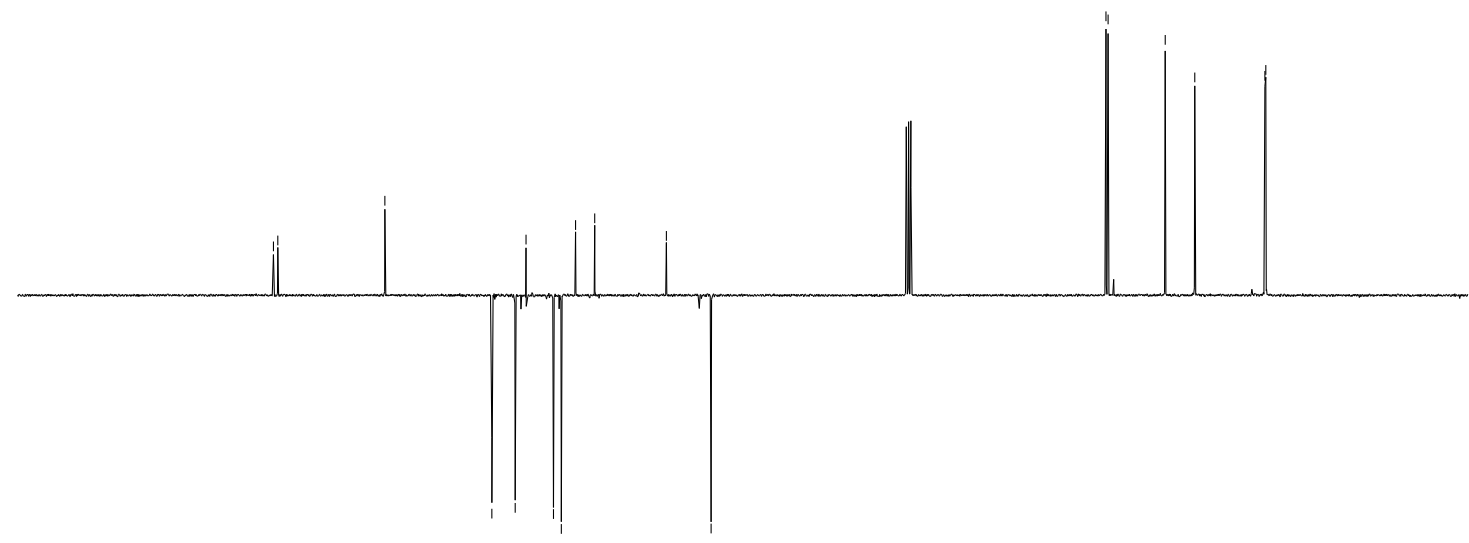

$\begin{array}{lllllllllllllllllllll}1 & 190 & 190 & 170 & 160 & 150 & 140 & 130 & 120 & 110 & 100 & 90 & 80 & 70 & 60 & 50 & 40 & 30 & 20 & 10 & 0\end{array}$

Figure S60. ${ }^{13} \mathrm{C}$ NMR (SEFT) spectrum of $N$-(2-azidopropyl)-4-((2-azidopropyl)amino)-1,8-naphtalimide in $\mathrm{CDCl}_{3}$. 


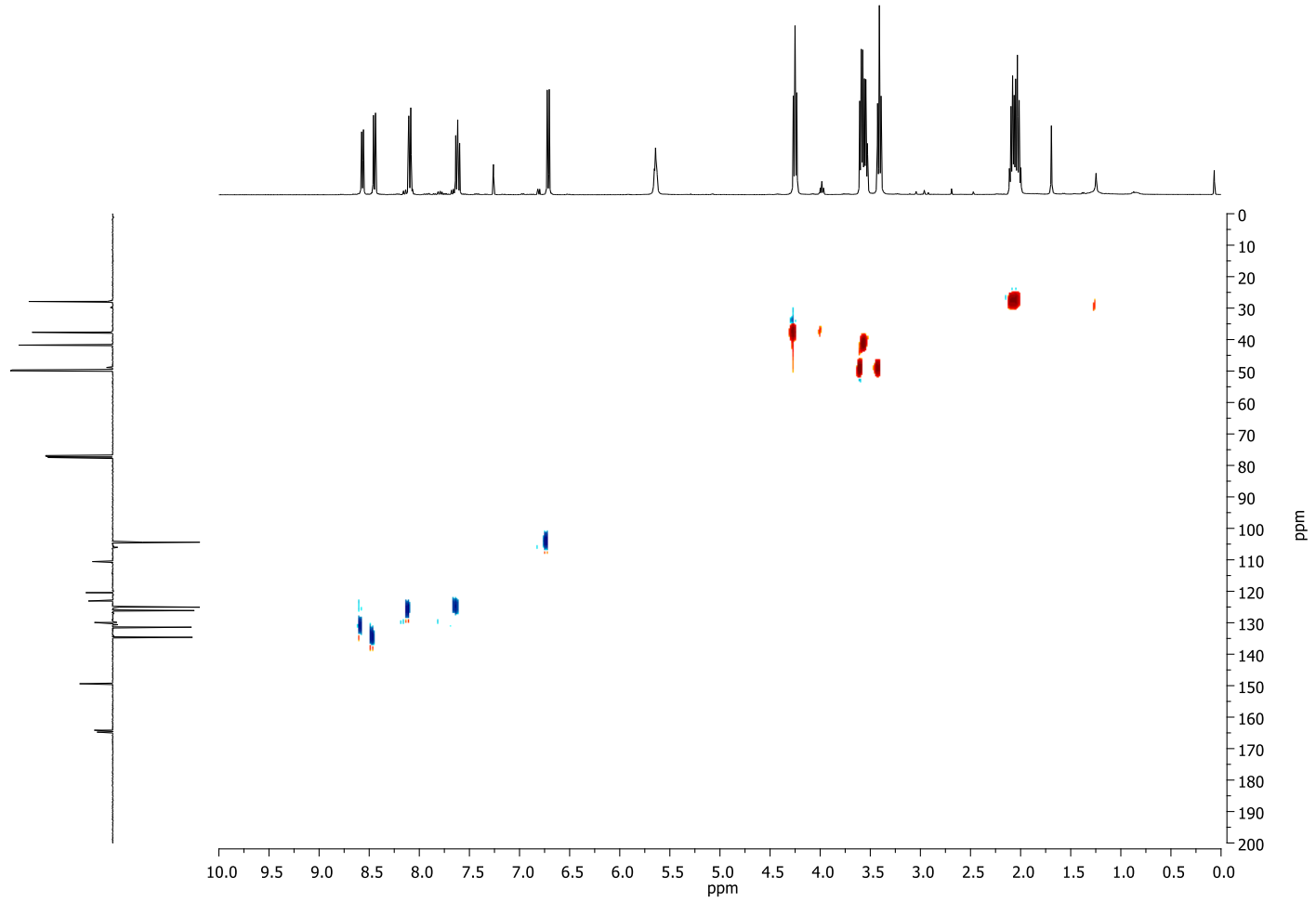

Figure S61. HSQC spectrum of $N$-(2-azidopropyl)-4-((2-azidopropyl)amino)-1,8-naphtalimide in $\mathrm{CDCl}_{3}$. 


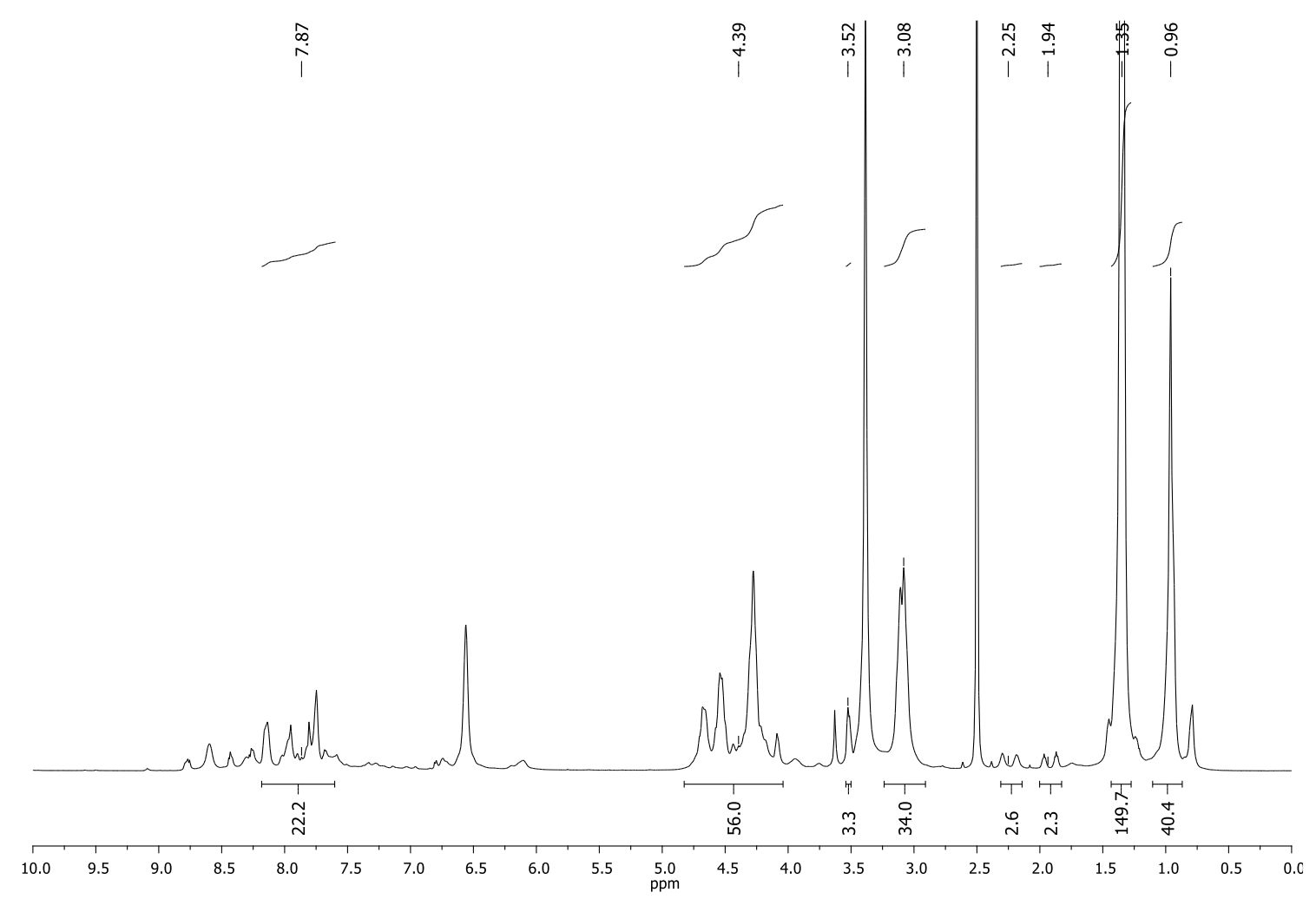

Figure S62. ${ }^{1} \mathrm{H}$ NMR spectrum of $\mathbf{G} \mathbf{3}_{\mathrm{Naph}} \mathbf{N H B o c}$ in DMSO- $d_{6}$.

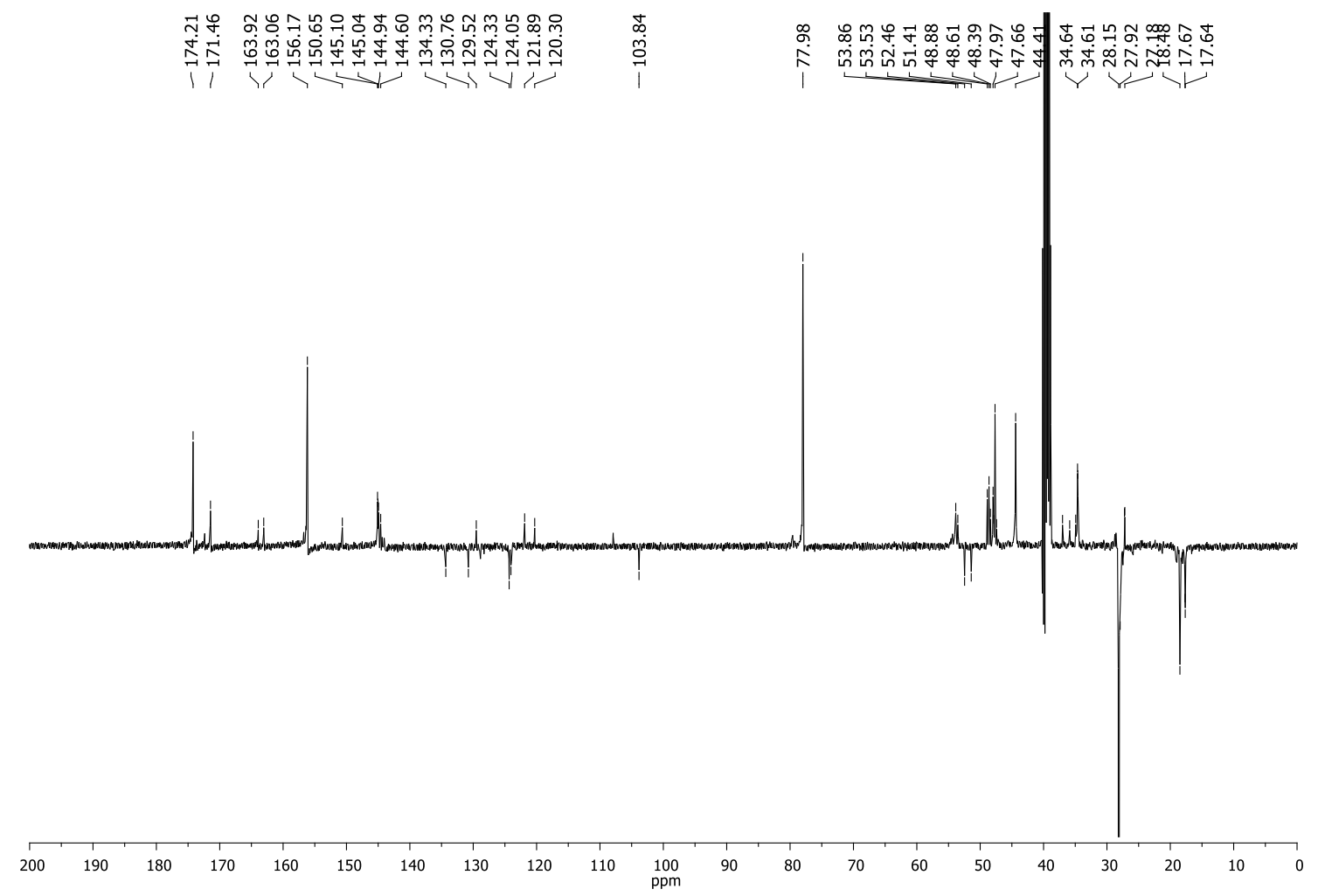

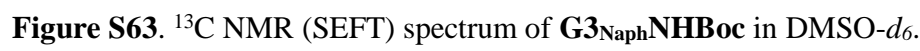




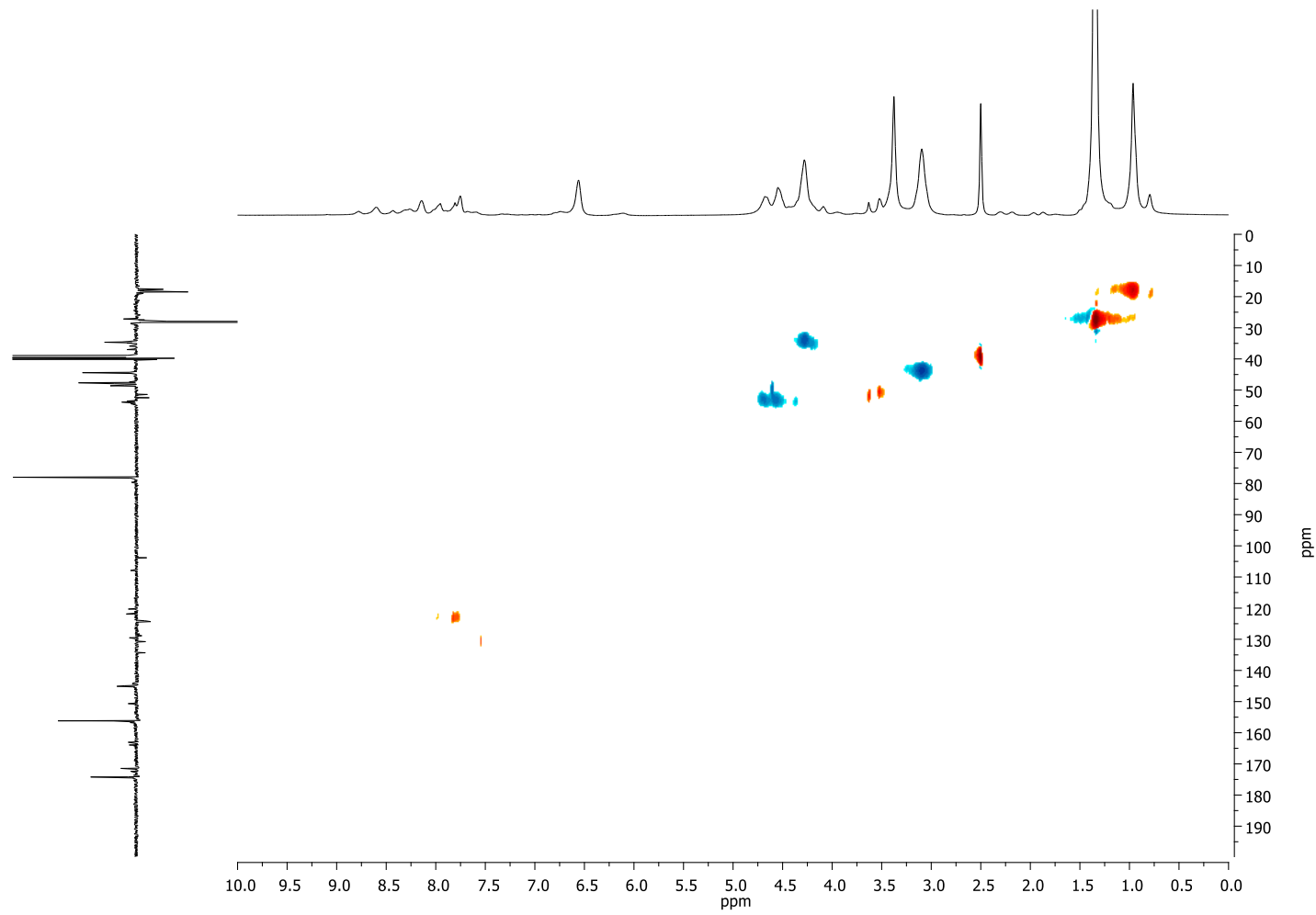

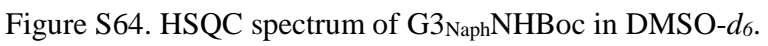

S-43 


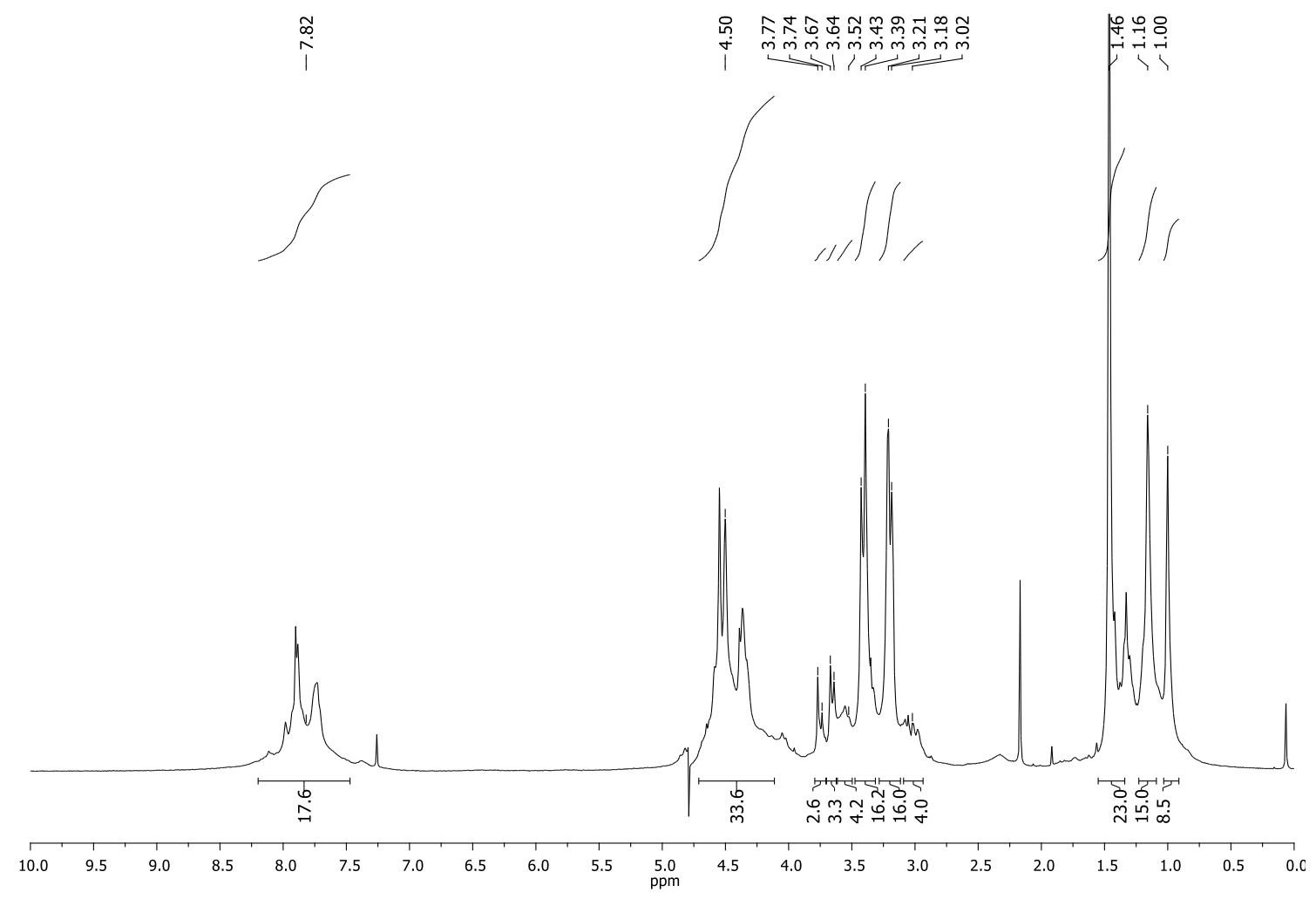

Figure S65. ${ }^{1} \mathrm{H}$ NMR spectrum of $\mathbf{G} \mathbf{N}_{\mathbf{N a p h}} \mathbf{N H}_{2}$ in $\mathrm{D}_{2} \mathrm{O}$.

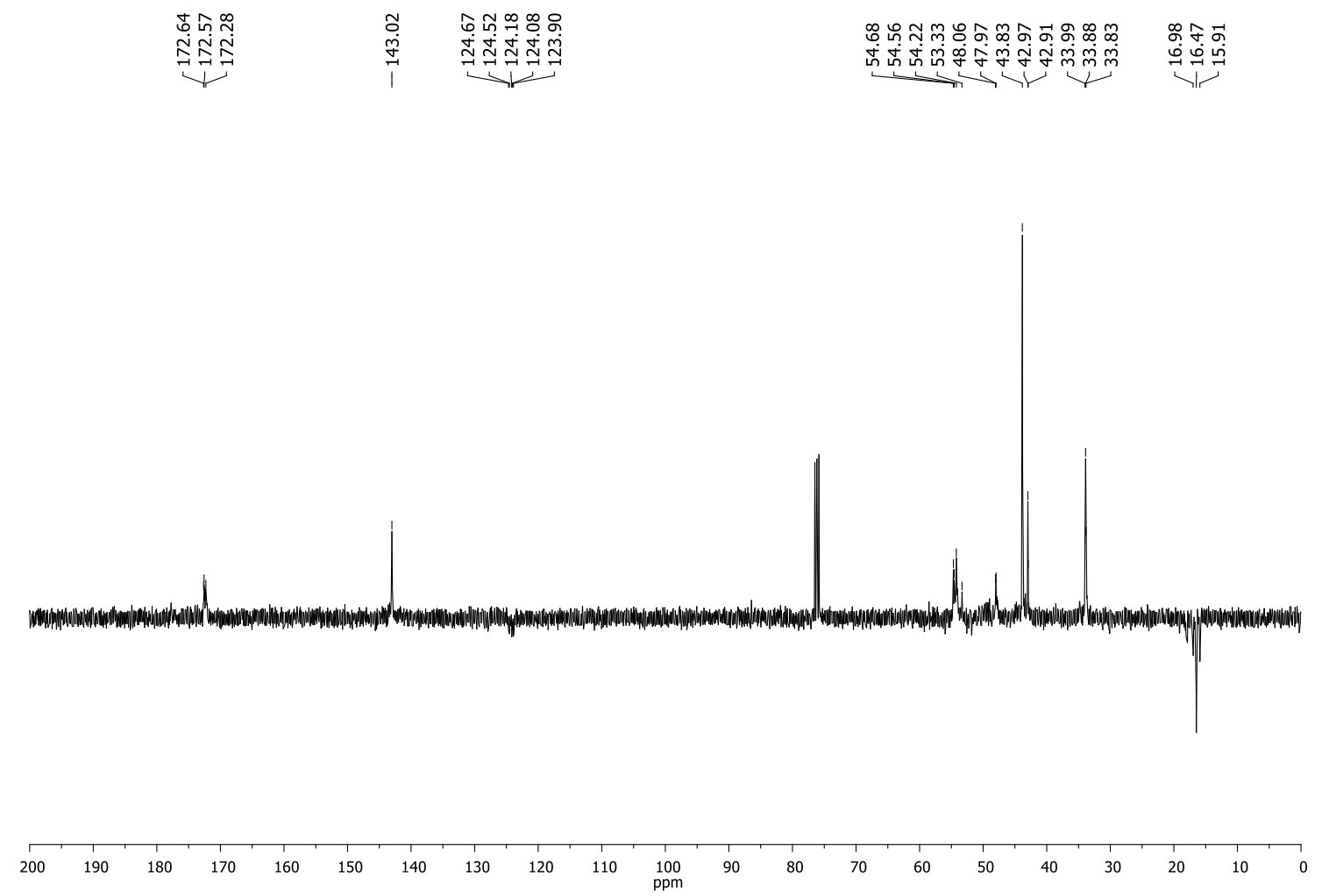

Figure S66. ${ }^{13} \mathrm{C}$ NMR (SEFT) spectrum of $\mathbf{G 3}_{\mathbf{N a p h}} \mathbf{N H}_{2}$ in $\mathrm{D}_{2} \mathrm{O}$. 


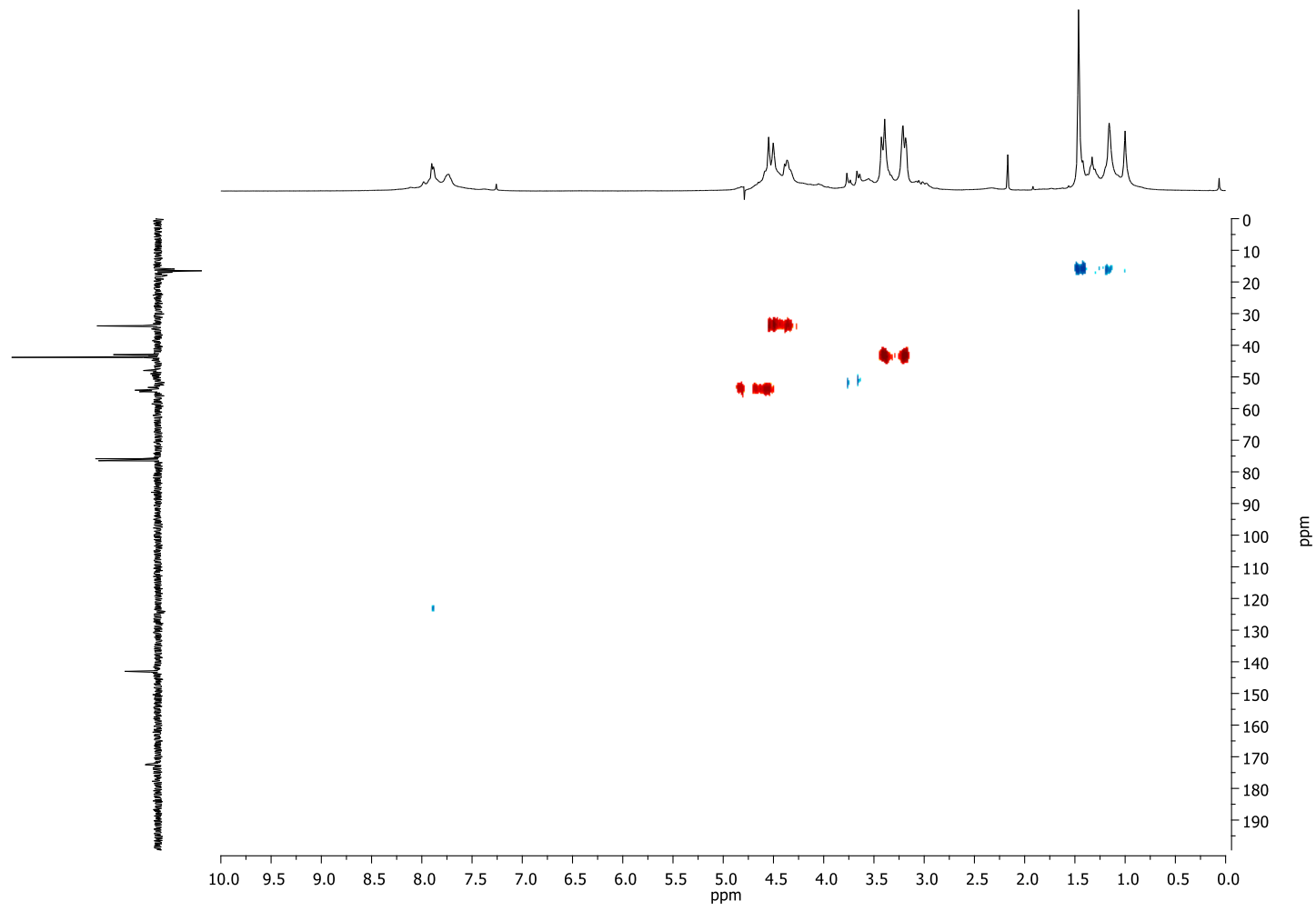

Figure S67. HSQC spectrum of $\mathrm{G}_{3} \mathrm{NaphNH}_{2}$ in $\mathrm{D}_{2} \mathrm{O}$. 


\section{DOSY NMR Experiments}

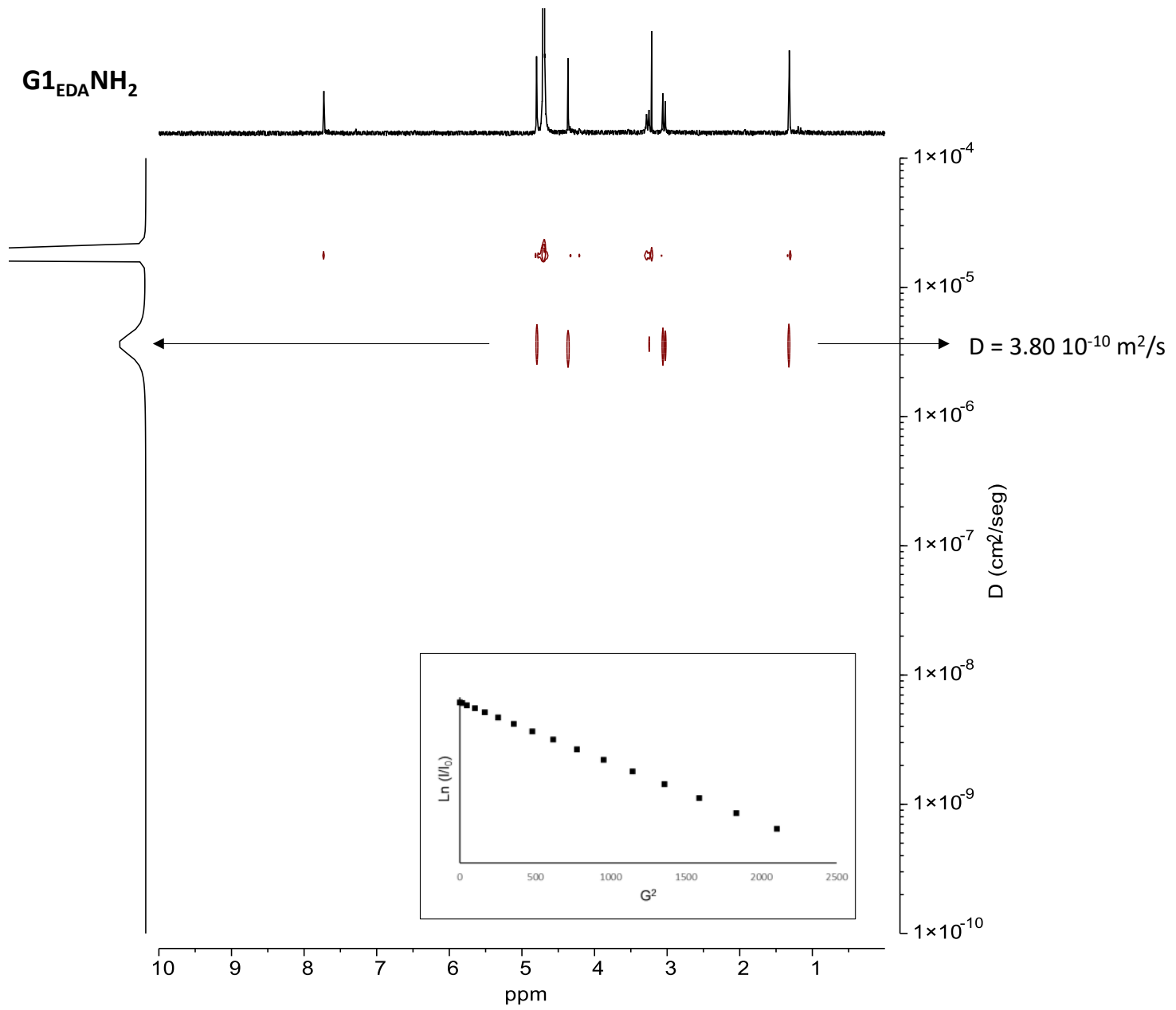

Figure S68. DOSY NMR experiment of $\mathbf{G 1}_{\mathbf{E D A}} \mathbf{N H}_{2}$ in $\mathrm{D}_{2} \mathrm{O}$. Inset, Stejskal-Tanner plot. 


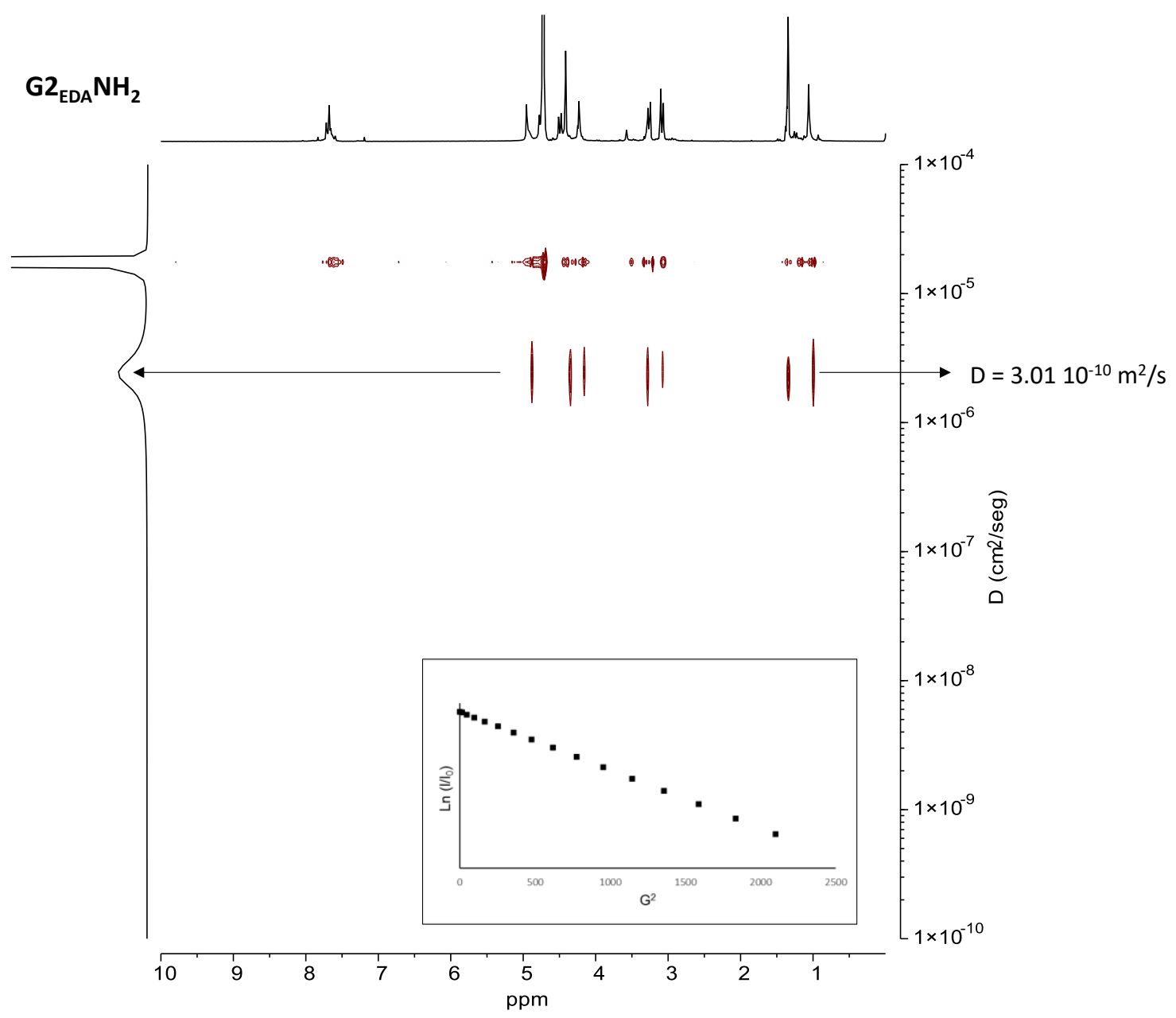

Figure S69. DOSY NMR experiment of G2 $\mathbf{E D A N H}_{2}$ in $\mathrm{D}_{2} \mathrm{O}$. Inset, Stejskal-Tanner plot. 


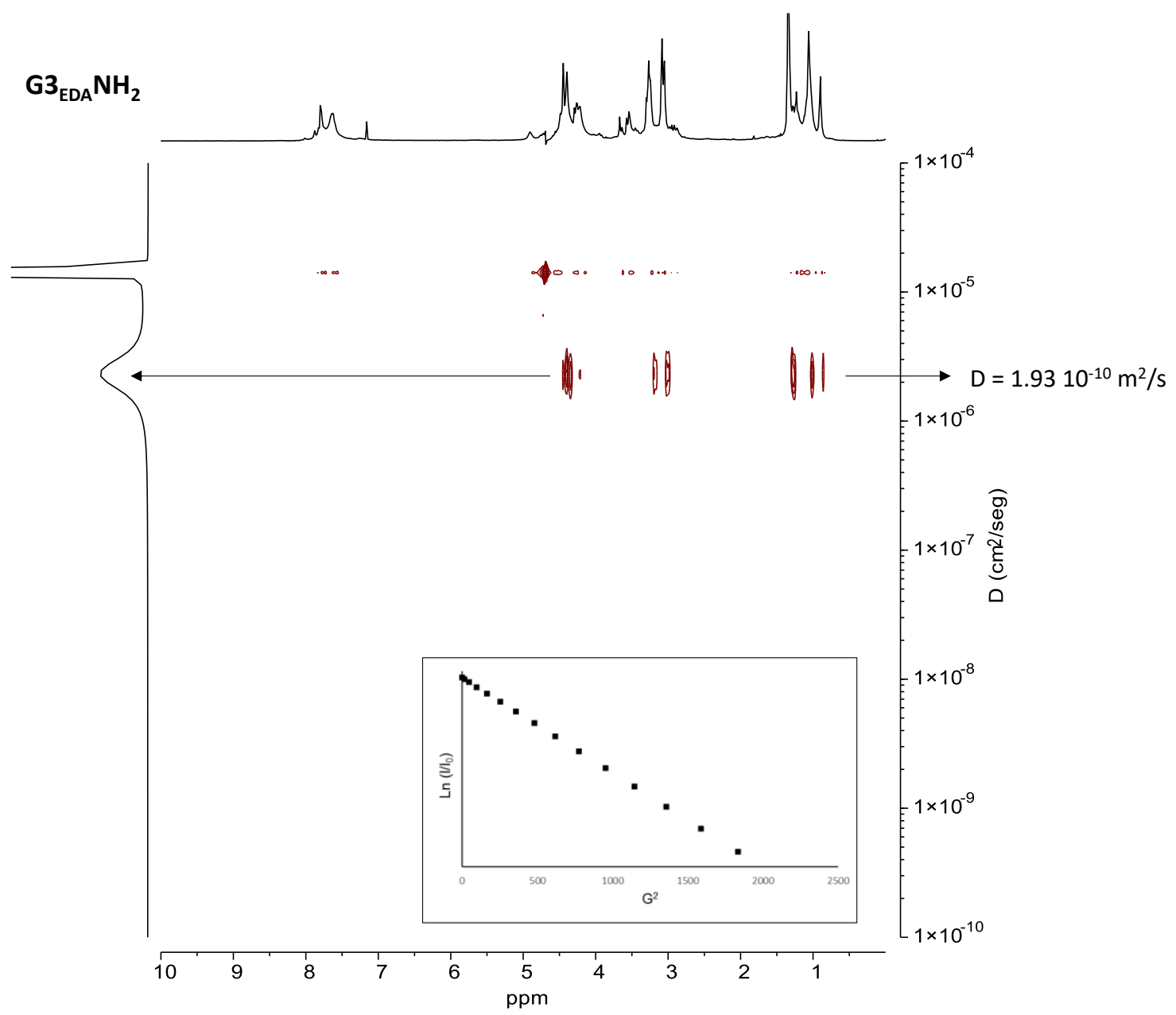

Figure S70. DOSY NMR experiment of $\mathbf{G 3}_{\mathbf{E D A N H}} \mathbf{N H}_{2}$ in $\mathrm{D}_{2} \mathrm{O}$. Inset, Stejskal-Tanner plot. 


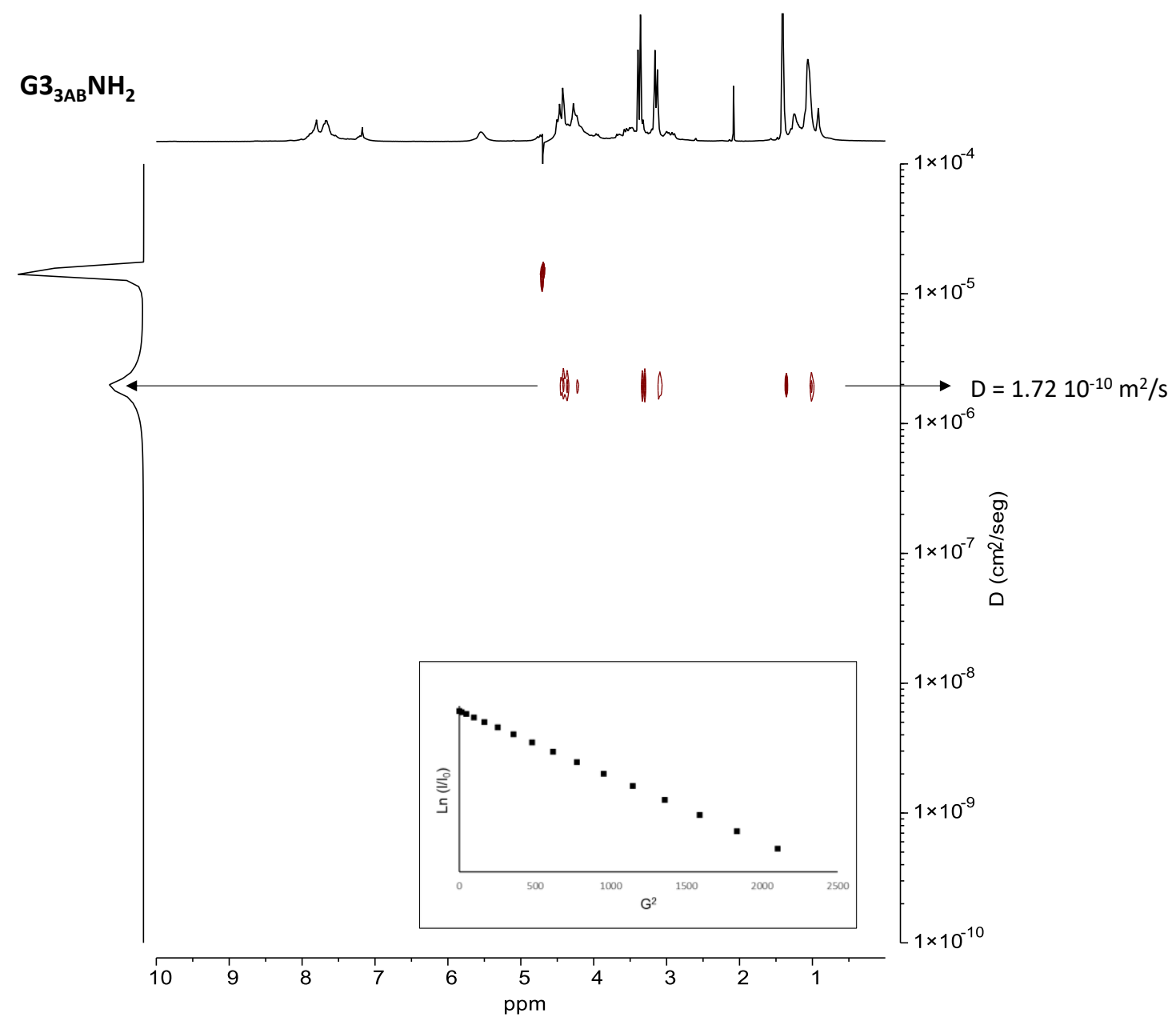

Figure S71. DOSY NMR experiment of $\mathbf{G 3}_{3} \mathbf{3 B}_{\mathbf{B}} \mathbf{N H}_{2}$ in $\mathrm{D}_{2} \mathrm{O}$. Inset, Stejskal-Tanner plot. 


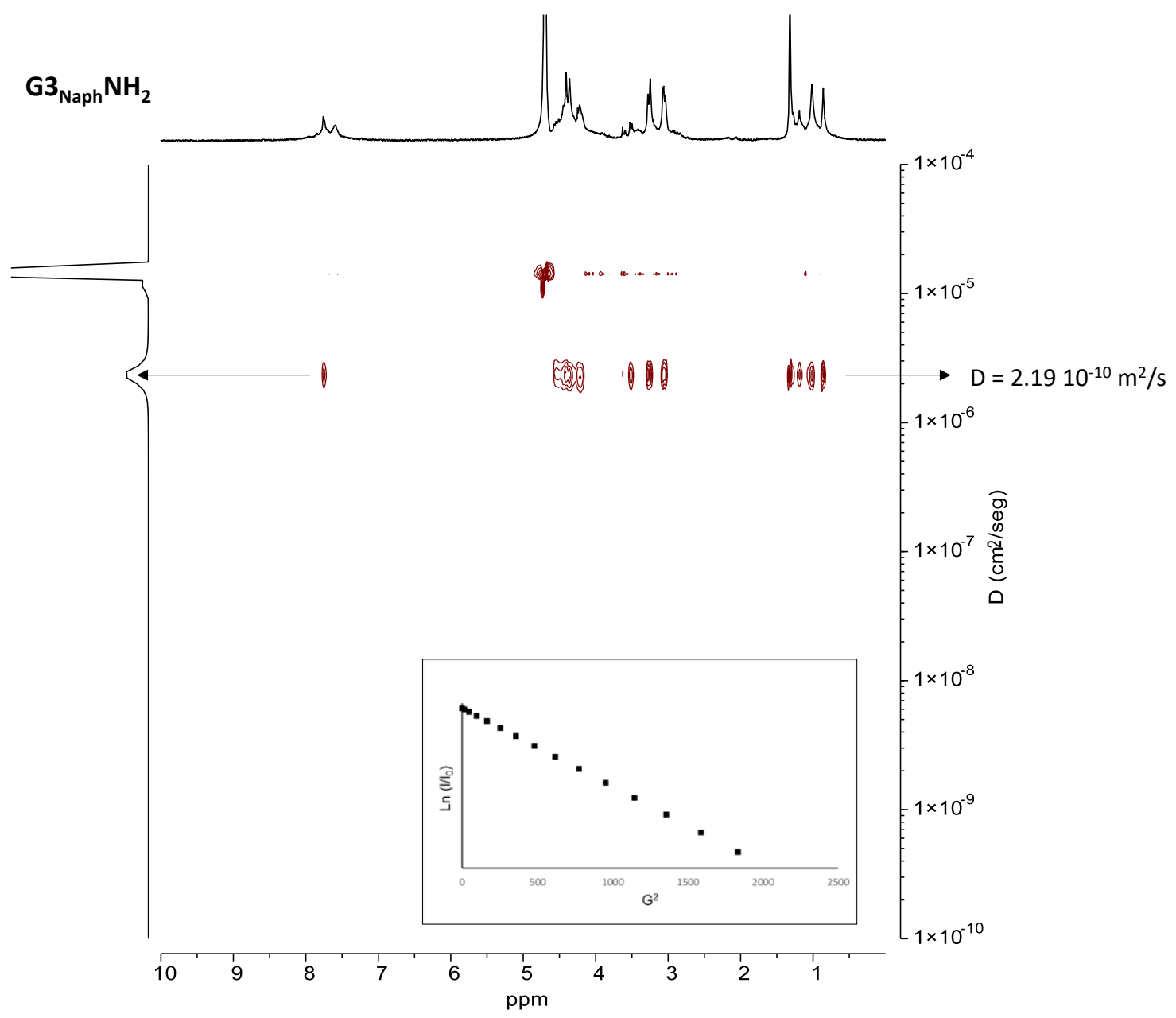

Figure S72. DOSY NMR experiment of $\mathbf{G 3}_{\mathbf{N a p h}} \mathbf{N H}_{2}$ in $\mathrm{D}_{2} \mathrm{O}$. Inset, Stejskal-Tanner plot. 


\section{IR spectra}

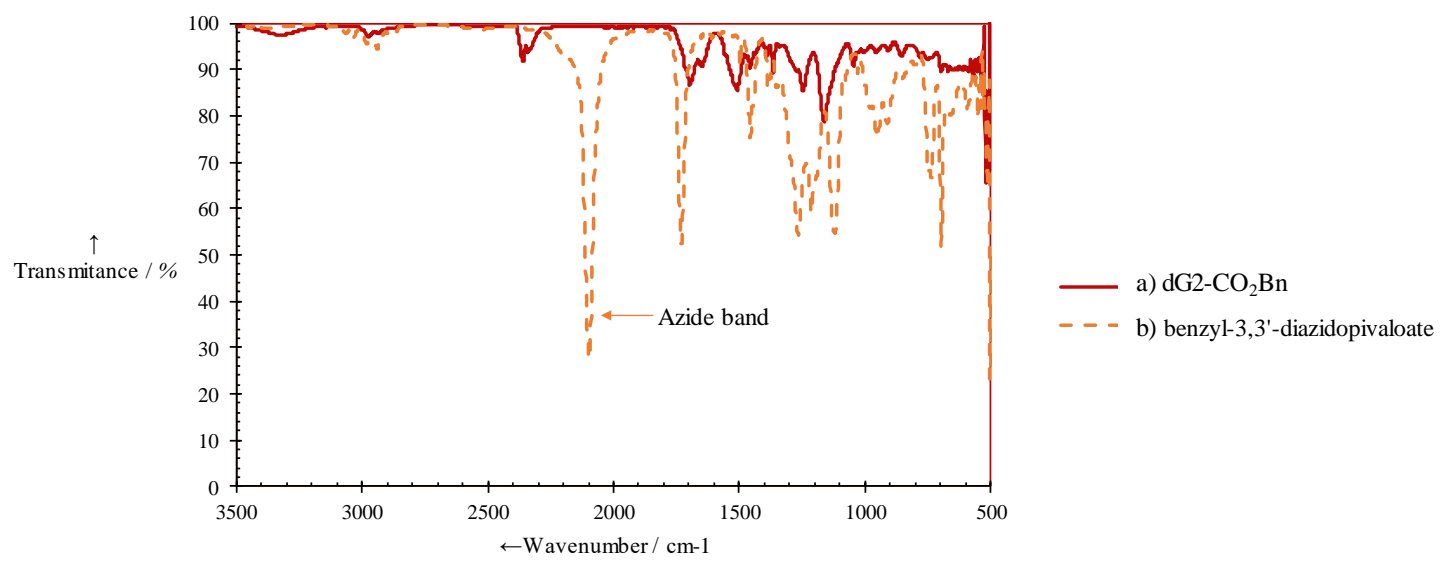

Figure S73. IR spectra of a) dG2-C $\mathbf{C O}_{2} \mathbf{B n}$ and b) benzyl-3,3'-diazidopivaloate.

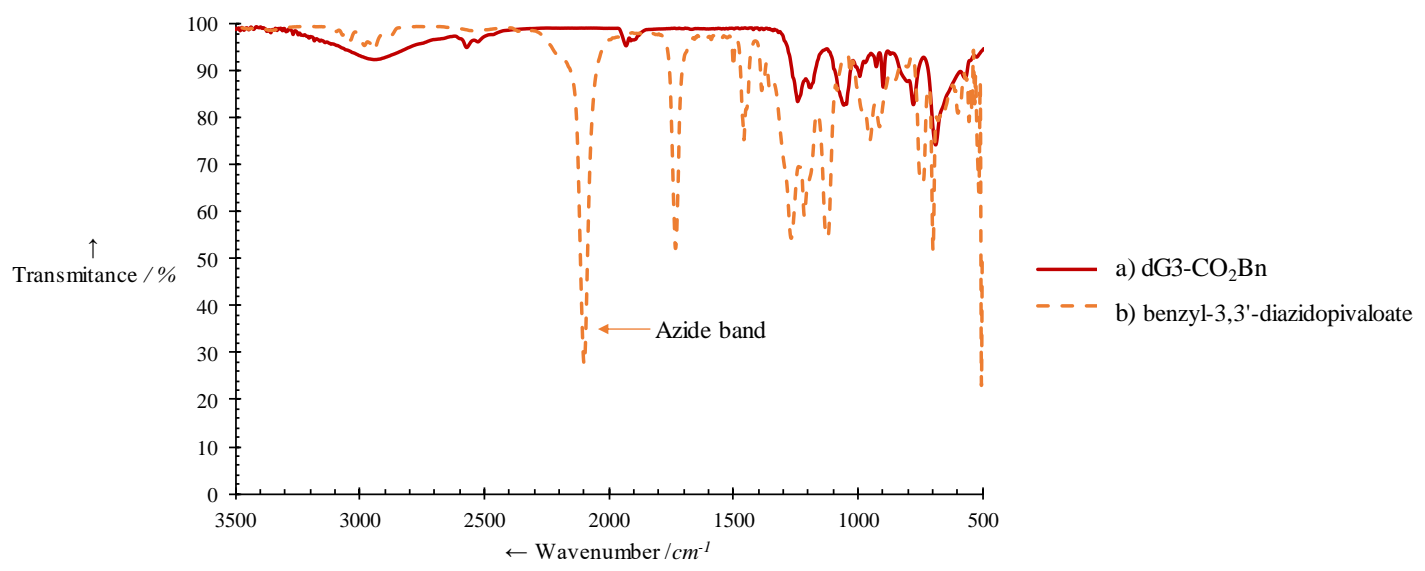

Figure S74. IR spectra of a) dG3- $\mathrm{CO}_{2} \mathbf{B n}$ and b) benzyl-3,3'-diazidopivaloate.

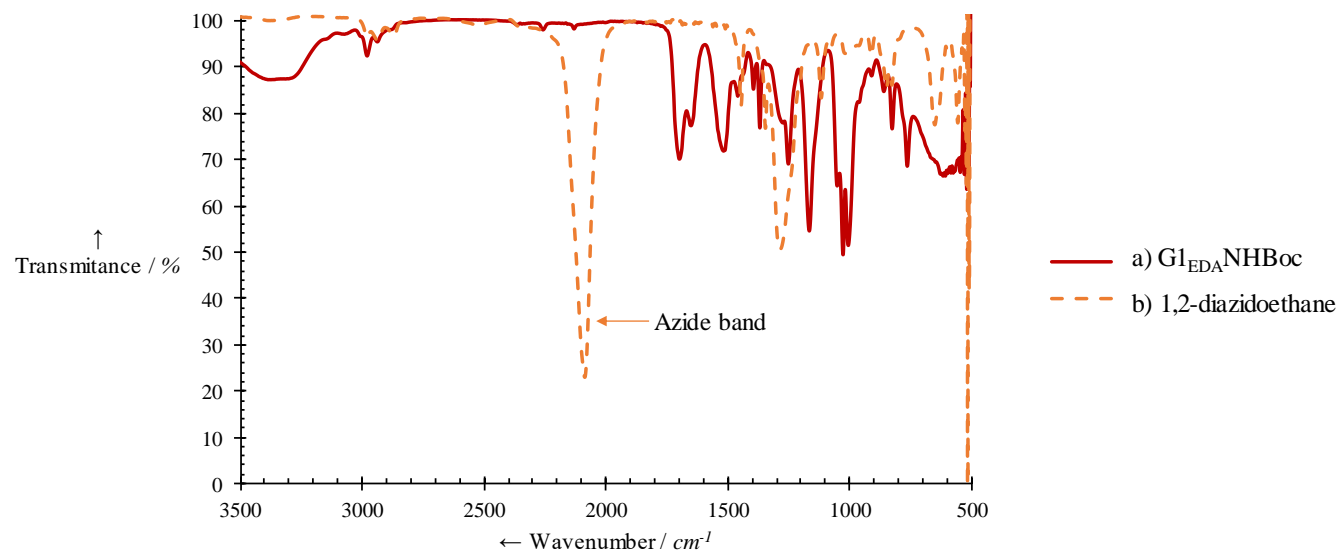

Figure S75. IR spectra of a) G1 EDANHBoc and b) 1,2-diazidoethane. 


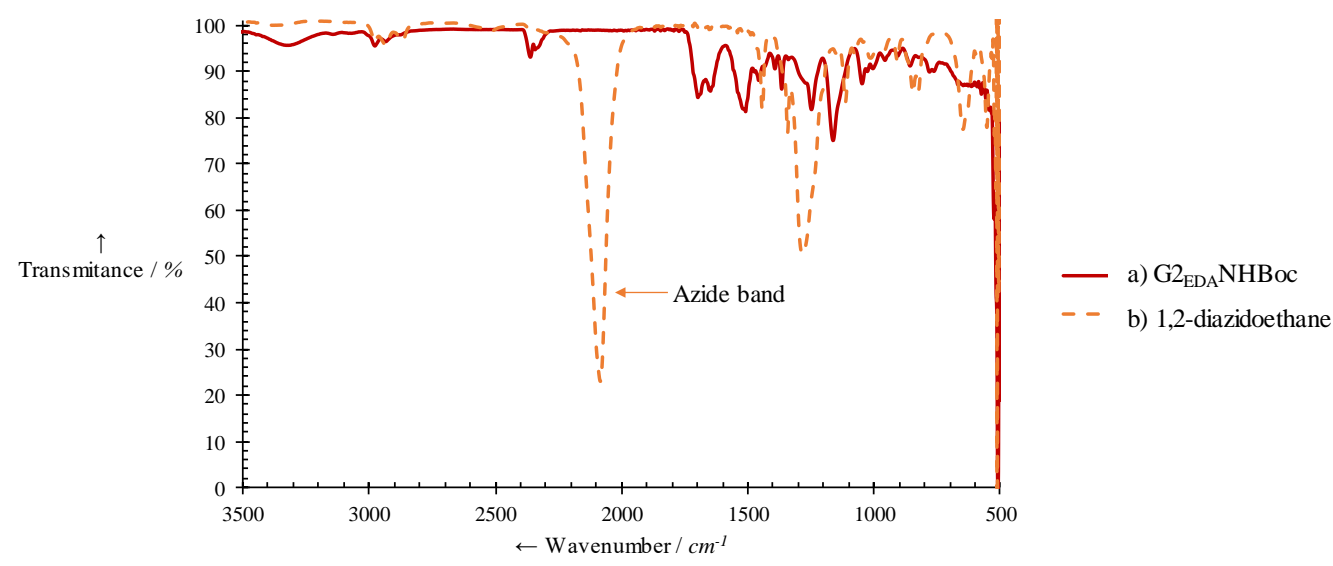

Figure S76. IR spectra of a) G2EDANHBoc and b) 1,2-diazidoethane.

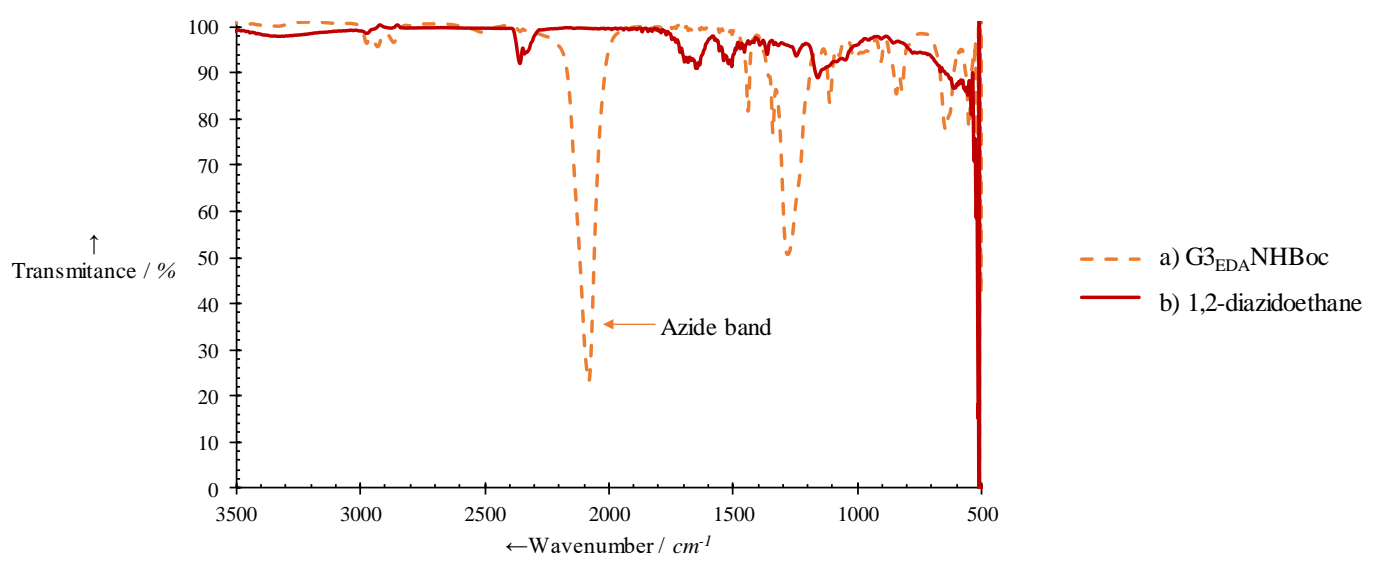

Figure S77. IR spectra of a) G3EDANHBoc and b) 1,2-diazidoethane.

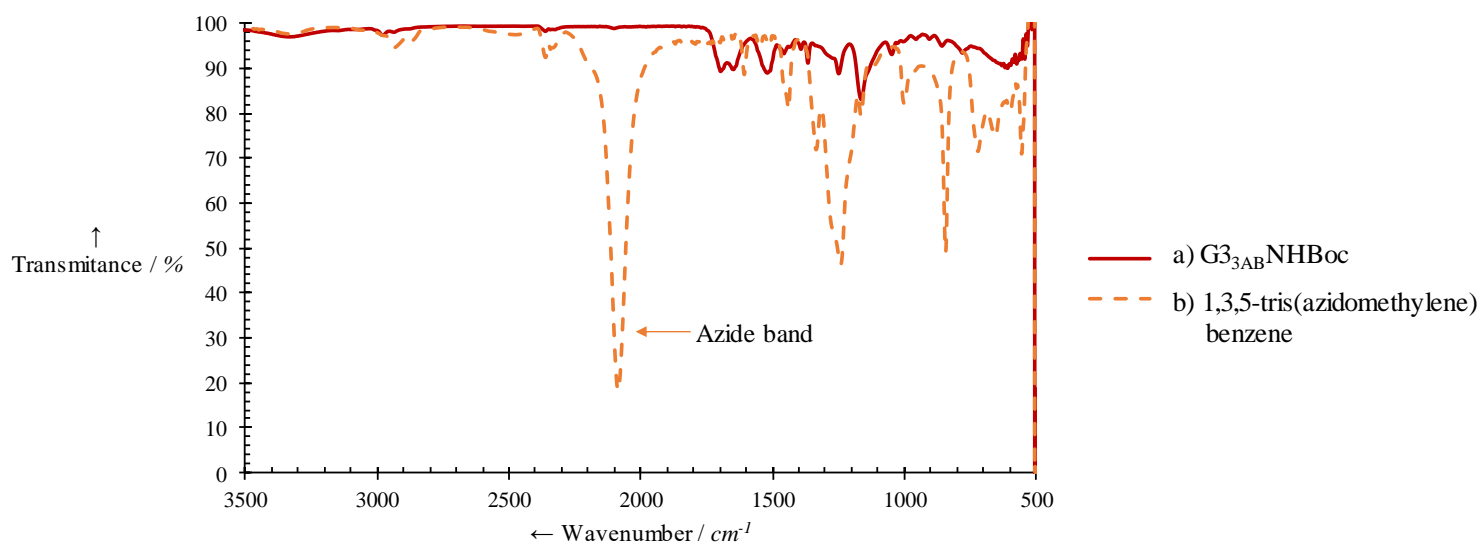

Figure S78. IR spectra of a) $\mathbf{G 3}_{3 \mathrm{AB}} \mathbf{N H B o c}$ and b) 1,3,5-tris(azidomethylene)benzene. 


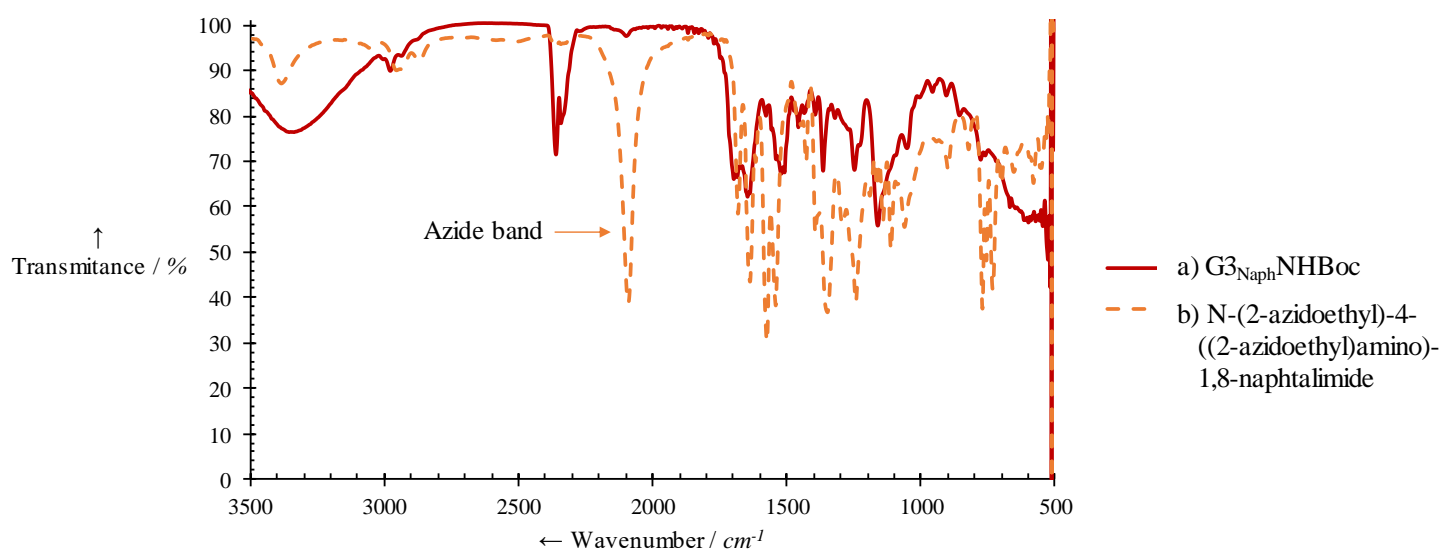

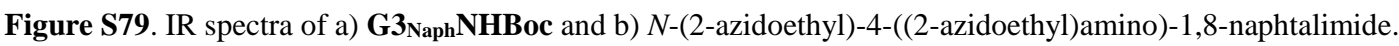




\section{Luminescent Properties of $\quad N$-(2-aminoethyl)-4-((2-aminoethyl)amino)-1,8- naphthalimide}

$N$-(2-aminoethyl)-4-((2-aminoethyl)amino)-1,8-naphthalimide has been prepared as described in literature. ${ }^{1}$

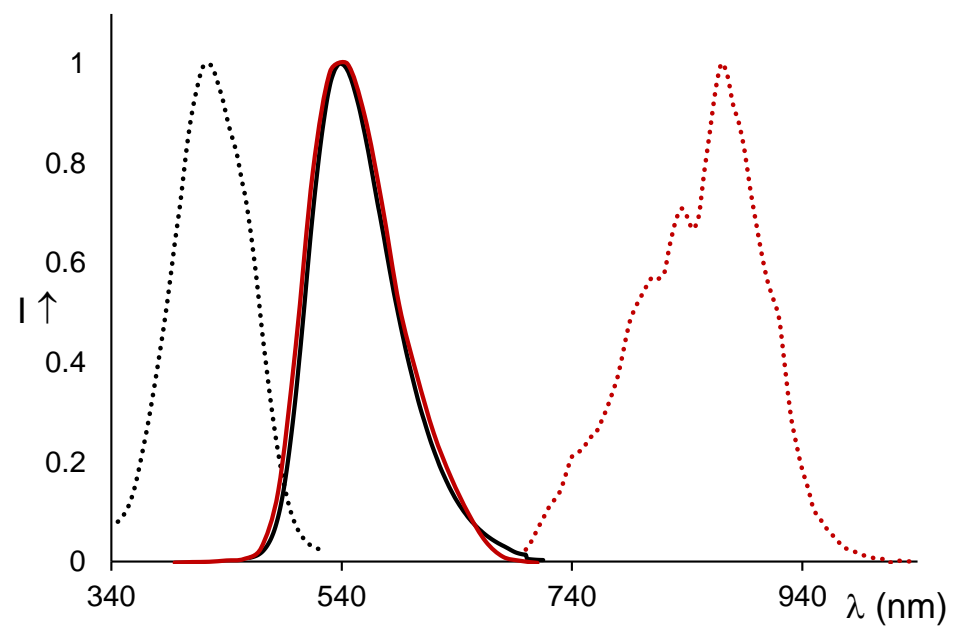

Figure S80. Excitation (dotted line) and emission (solid line) spectra of $\mathbf{N}$-(2-aminoethyl)-4-((2-aminoethyl)amino)1,8-naphthalimide upon one-photon (black) or two-photon (red) excitation (440 nm and $830 \mathrm{~nm}$ respectively), in 10-5 $\mathrm{M}$ aqueous solutions.

Luminescent Microscopy experiments with $E$. coli bacteria incubated with G3 ${ }_{\text {NaphNH2 }}\left(5 \cdot 10^{-4} \mathrm{M}\right)$
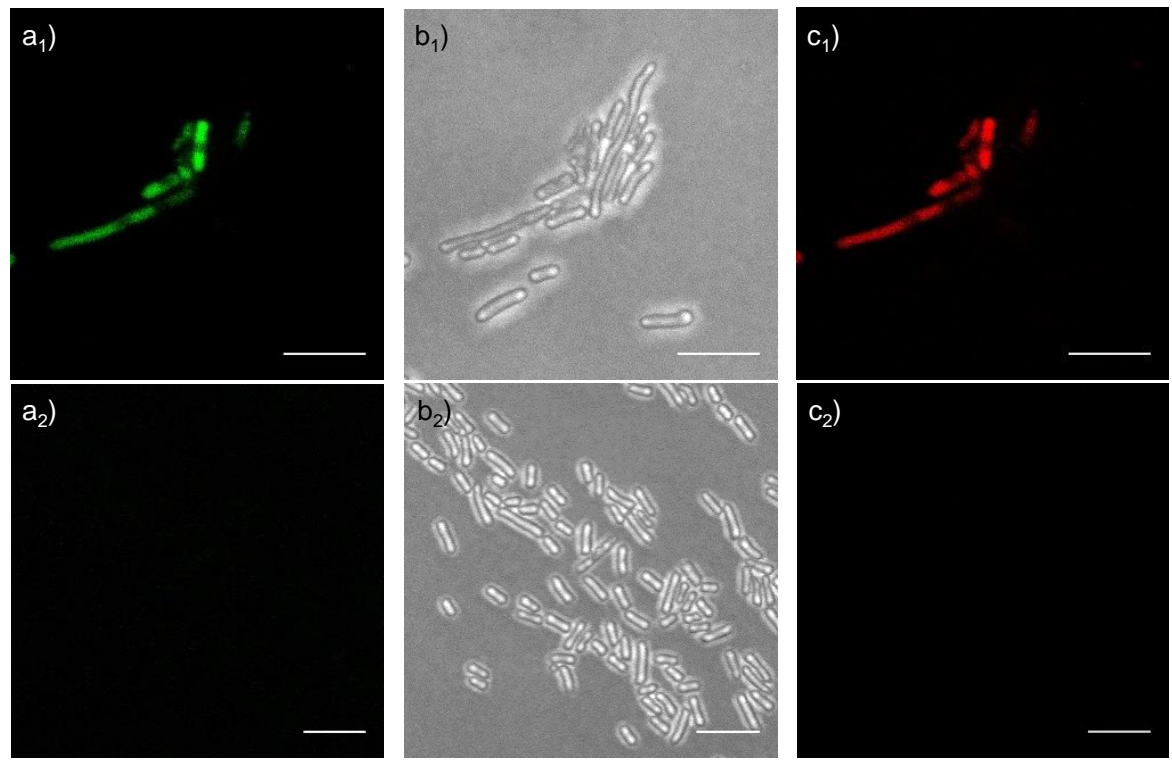

Figure S81. Confocal micrographs of $E$. coli incubated for $8 \mathrm{~h}$ with (upper row; $\mathrm{a}_{1}, \mathrm{~b}_{1}$ and $\mathrm{c}_{2}$ ) or without (bottom row; $\mathrm{a}_{2}, \mathrm{~b}_{2}$ and $\left.\mathrm{c}_{2}\right) \mathbf{G 3}_{\mathrm{Naph}_{\mathbf{N H}}} \mathbf{N H}_{2}$ : $\left.\mathrm{a}_{1}, \mathrm{a}_{2}\right)$ recorded emission with one photon excitation $\left(\lambda_{\text {exc }}=450 \mathrm{~nm}\right.$; collected through 500 $\left.600 \mathrm{~nm}) ; b_{1}, b_{2}\right)$ bright field images; $\left.c_{1}, c_{2}\right)$ recorded emission with two photon excitation $\left(\lambda_{\text {exc }}=880 \mathrm{~nm}\right.$; collected through 500-550 nm). Scale Bars: $5 \mu \mathrm{m}$. 


\section{Theoretical Calculations}

Dendrimer Building. Dendrimers are composed of three different residues: the core (COR) for the corresponding dendrimers, the repetitive 3,3'-[(4-(aminomethyl)-1H-1,2,3-triazol1-yl)]-2-methylpropanoyl units (REP), and the terminal end 3,3'-diamoniopivaloyl unit (TAM) (Figure S82).

For all of these residues the cap region was removed before connecting to another residue. For the 1,4-substituted triazole group we used the parameter described before. ${ }^{2}$ The values (bonds, angle torsions, or van der Waals parameters) not included in the parm99 force field, were obtained from the general AMBER force field (GAFF). ${ }^{3}$ The minimum energy conformation was submitted to B3LYP/6-31G(d) basis set calculation using G09, ${ }^{4}$ (capped COR and REP residues were optimized in vacuum, while capped TAM residue was optimized employing PCM-B3LYP/6-31G(d) with water as solvent). ${ }^{5}$ The restrained potential (RESP) method was used for charge fitting. ${ }^{6}$ Total cap atom charge was constrained to zero during charge calculation and the overall full residue charge was also set to zero except for the protonated residue (TAM), which was kept at +2 . Decapped residues were created using the Antechamber module of AmberTools 12, ${ }^{7}$ and used to build the desired dendrimer generation for the GnEDANH2 with the Dendrimer Building Tool (DBT). ${ }^{8}$ The $\mathbf{G} 33_{3 A B} \mathbf{N H}_{2}$ and $\mathbf{G} 3_{\text {Naph }} \mathbf{N H}_{2}$ dendrimers were built with AmberTools 12 and the LEaP package. 

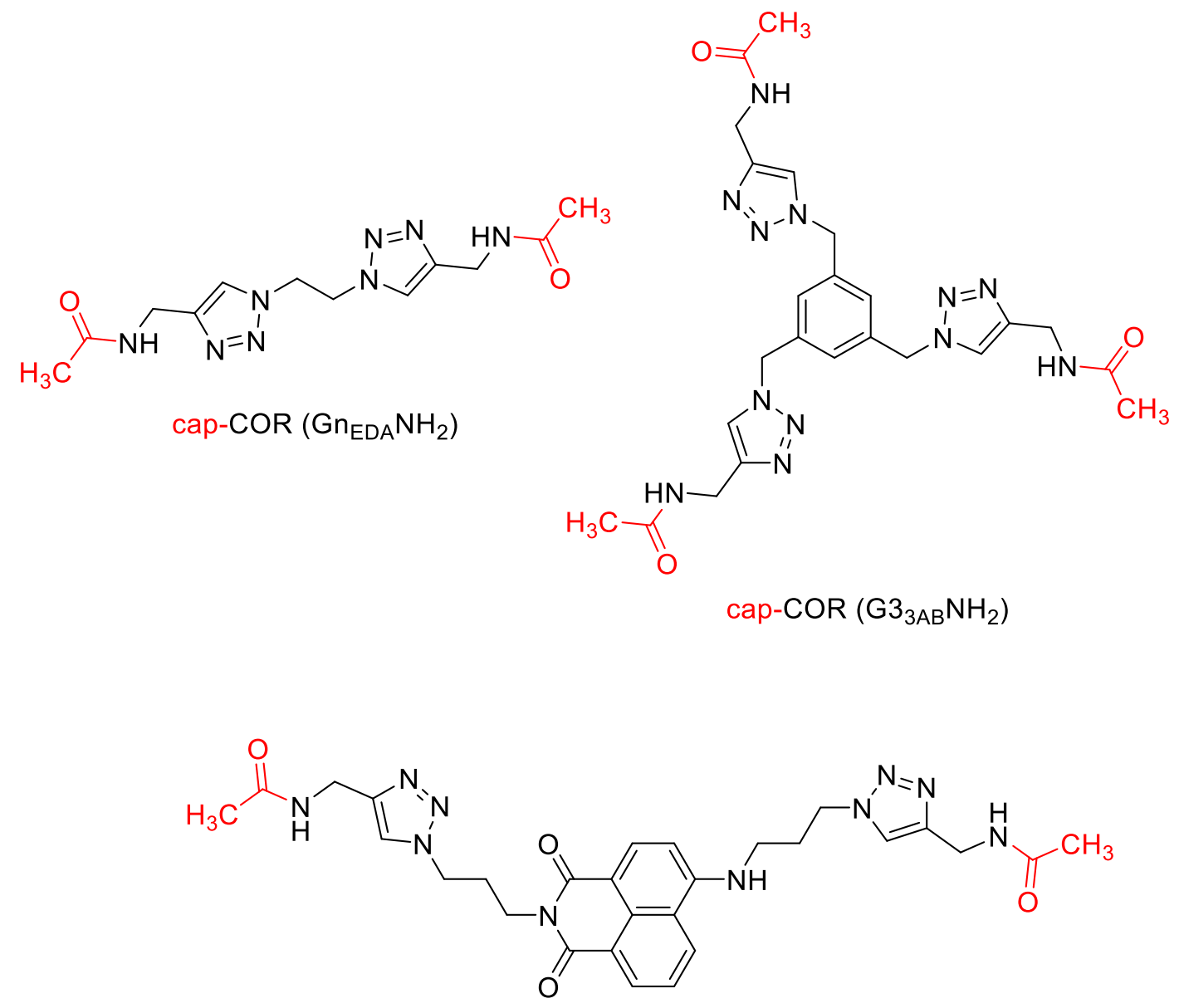

cap-COR ( $\left(3_{\mathrm{Naph}} \mathrm{NH}_{2}\right)$

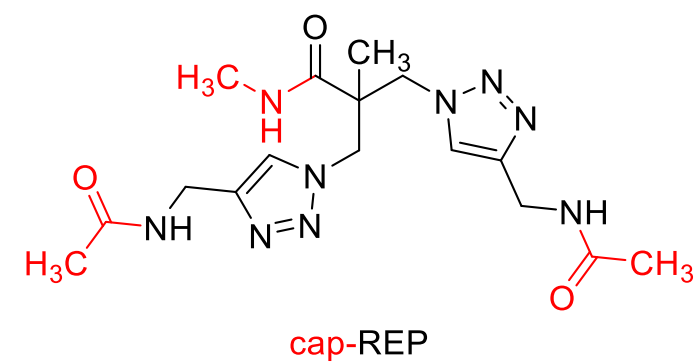<smiles>CNC(=O)C(C)(C[NH3+])C[NH3+]</smiles>

cap-TAM

Figure S82. Residues selection for the dendrimers. Cap atoms are in red.

Simulation Details. Simulations were performed in water as explicit solvent at neutral pH.

We used the AMBER 12 MD software package for all calculations. ${ }^{7}$ An appropriate number of $\mathrm{Cl}^{-}$counterions were added, to preserve overall charge, and the molecules hydrated, using the TIP3P water model, ${ }^{9}$ in truncated octahedral cells. In all cases, a 
minimum $10 \AA$ solvation shell around the dendrimer structure were chosen to provide the dimensions of these cells.

\begin{tabular}{llllll}
\hline Dendrimer & $N_{\text {den }}$ & $N_{\mathrm{Cl}}^{-}$ & $N_{\text {solvent }}$ & $N_{\text {total }}$ & $V\left(\AA^{3}\right)$ \\
\hline G1EDANH2 & 70 & 4 & 8013 & 8087 & 93214 \\
G2EDANH2 & 182 & 8 & 12756 & 12946 & 150013 \\
G3EDANH2 & 406 & 16 & 16479 & 16901 & 102339 \\
\hline G3NaphNH2 & 440 & 16 & 25008 & 25464 & 288741 \\
\hline G33ABNH2 & 618 & 24 & 25422 & 26064 & 301943
\end{tabular}

Table S1. Initial properties of dendrimers and simulation details. $N_{\mathrm{den}}, N_{\mathrm{Cl}^{-}}, N_{\text {solvent }}$ and $N_{\text {total }}$ are, respectively, the number of dendrimer atoms, chloride ions, atoms in solvent molecules and the total number of atoms. $\mathrm{V}$ is the initial octahedral box volume.

Solvated structures were minimized as described previously. ${ }^{10}$ Briefly, the molecules were optimized using six cycles of conjugated gradient minimization. In the initial cycle, dendrimers were kept in their starting conformation with a harmonic constraint force. Then, was followed by another five periods of minimization decreasing the harmonic restraint force constant from $20 \mathrm{kcal} / \mathrm{mol}-\AA^{2}$ to zero.

The minimized structures were heated from 0 to $300 \mathrm{~K}$ with three steps of 40 ps of MD, the first of them under conditions of constant volume-constant temperature (NVT) and the rest under constant pressure-constant temperature (NPT) conditions. Finally, we carried out an unconstrained MD simulation (2ns) in NPT ensemble to equilibrate the system at $300 \mathrm{~K}$. The motion equation was solved using the Verlet leapfrog algorithm, ${ }^{11}$ with an integration step of 2 fs. The SHAKE algorithm was used to constrained the bond lengths involving bonds to hydrogen. ${ }^{12}$

Finally, production runs of $20 \mathrm{~ns}$ trajectories were done under an NPT ensemble. Temperature regulation was achieved using the Berendsen weak coupling. ${ }^{13}$ The particle- 
mesh Ewald (PME) algorithm was employed to treat long-range electrostatic interactions, ${ }^{14}$ with a real space cut off of $9 \AA$. The same cutoff was used for van der Waals interactions. For the structural analyses $\left(R_{g}, \mathrm{RDF}\right.$, etc. $)$ the last $1 \mathrm{~ns}$ equilibrated trajectory was used. Amber modules ptraj and cpptraj were used to accomplish these analyses. VMD software was used for the calculation of molecular surfaces. ${ }^{15}$

Dendrimers shape: Considering the average values of the three principal moments of inertia $I_{x}, I_{y}, I_{z}$ (in decreasing order) and the asphericity, a structure-persistent characteristic of these dendrimers can be obtained.

Molecular asphericity was calculated as defined: ${ }^{16}$

$$
\delta=1-3 \frac{\left\langle I_{2}\right\rangle}{\left\langle I_{1}^{2}\right\rangle}
$$

where $I_{1}$ and $I_{2}$ are the first and second invariants of the radius of gyration tensor: $I_{1}=I_{x}$ $+I_{y}+I_{z}, I_{2}=I_{x} I_{y}+I_{y} I_{z}+I_{x} I_{z}$. This quantity assumes values between 1 (for a linear array of atoms) and 0 (for shapes of high 3D similarity).

Monomer radial distribution functions: The average radial distribution function $\rho(r)$ can be expressed conveniently the dendrimers conformations. This radial function can be defined by counting the number $N(r)$ of atoms whose centers of mass are located within the spherical shell of radius $r$ and thickness $\Delta r$. Hence, integration over $r$ yields the total number of atoms as: $N(r)=4 \pi \int_{0}^{\infty} r^{2} \rho(r) d r$, where $r$ is the distance from the dendrimer center of mass. 
Fractal dimension ( $\left.d_{f}\right)$ for $\mathrm{Gn}_{E D A} \mathrm{NH}_{2}$

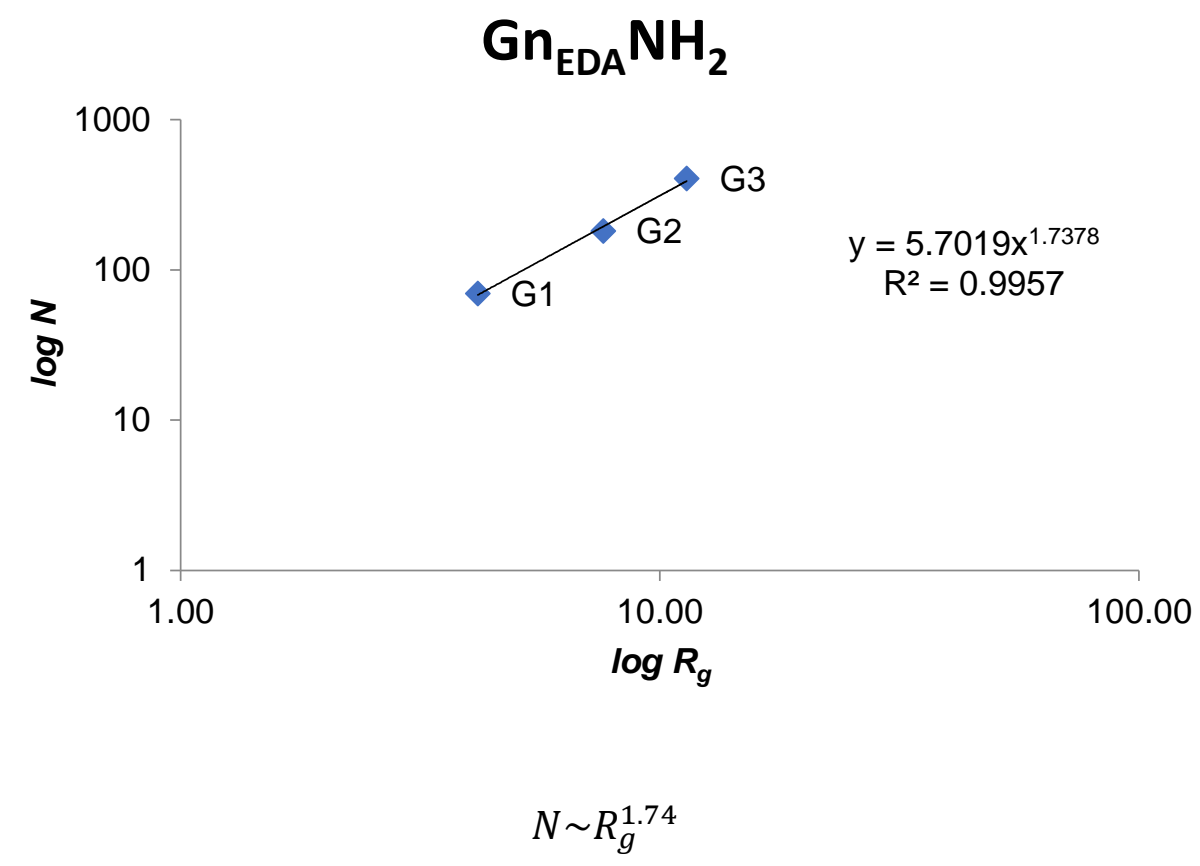

Figure S83. Relationship between the number of atoms $(N)$ of the $\mathbf{G n}_{\mathbf{E D A}} \mathbf{N H}_{\mathbf{2}}$ dendrimers and their $R_{g}$ values.
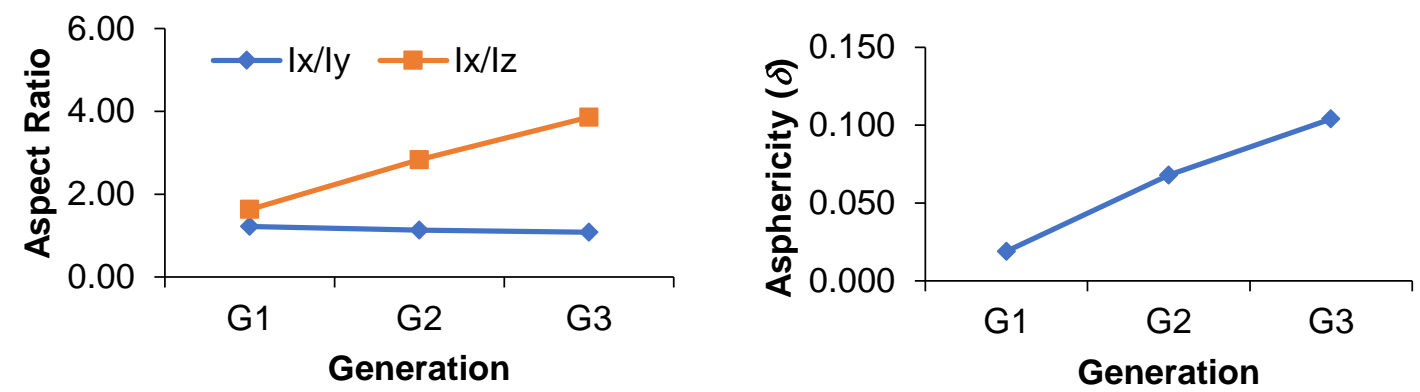

Figure S84. Aspect ratios and asphericity values for $\mathbf{G n}_{\mathbf{E D A}} \mathbf{N H}_{2}$ dendrimers as function of their generation. 


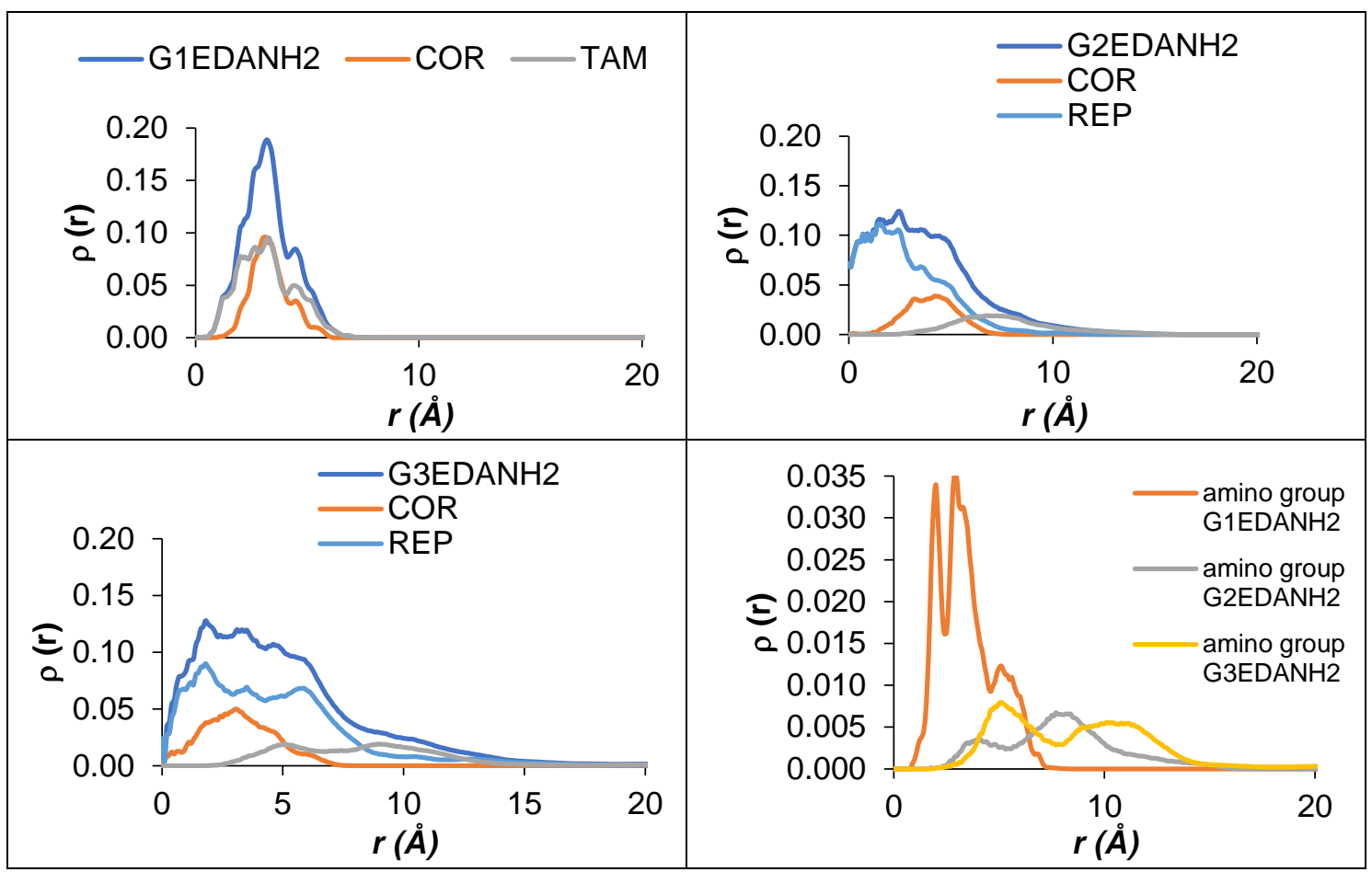

Figure S85. Radial distribution function of the $\mathbf{G n}_{\mathbf{E D A}} \mathbf{N H}_{2}$ dendrimers and its monomers using dendrimer center of mass as reference. The unit value for $\rho(r)$ is expressed in atoms $/ \AA^{3}$.
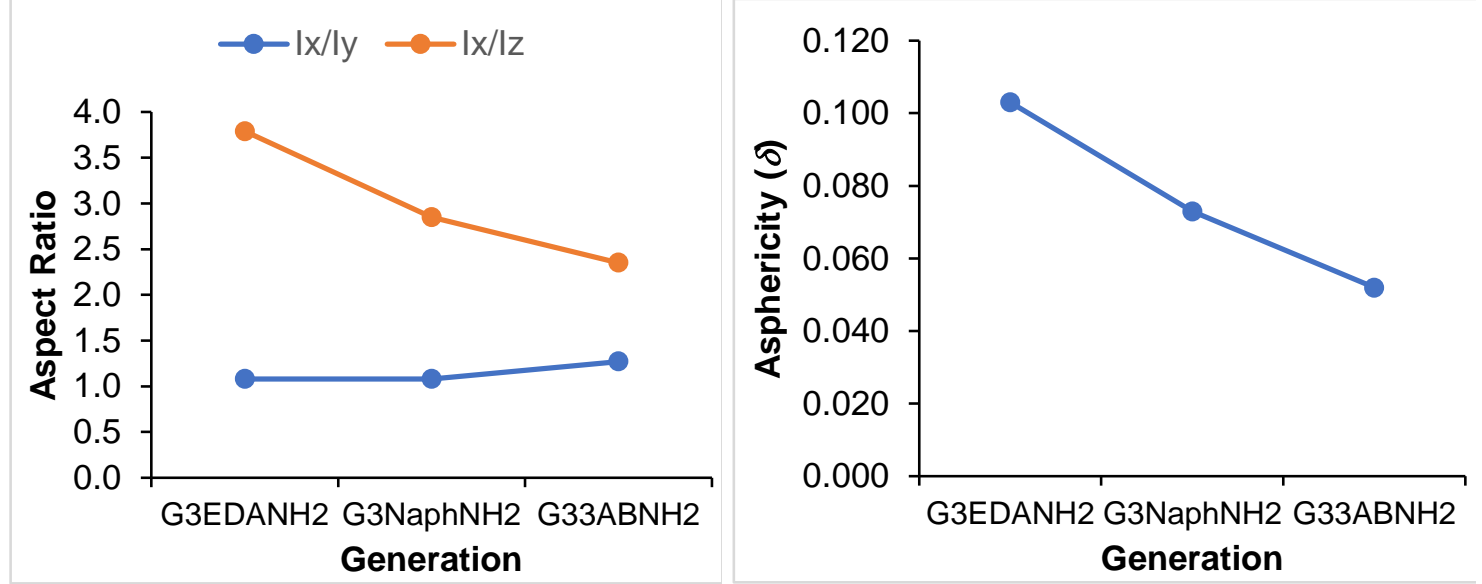

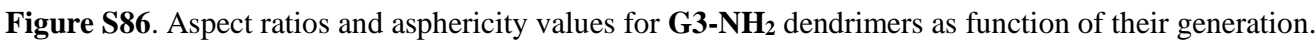




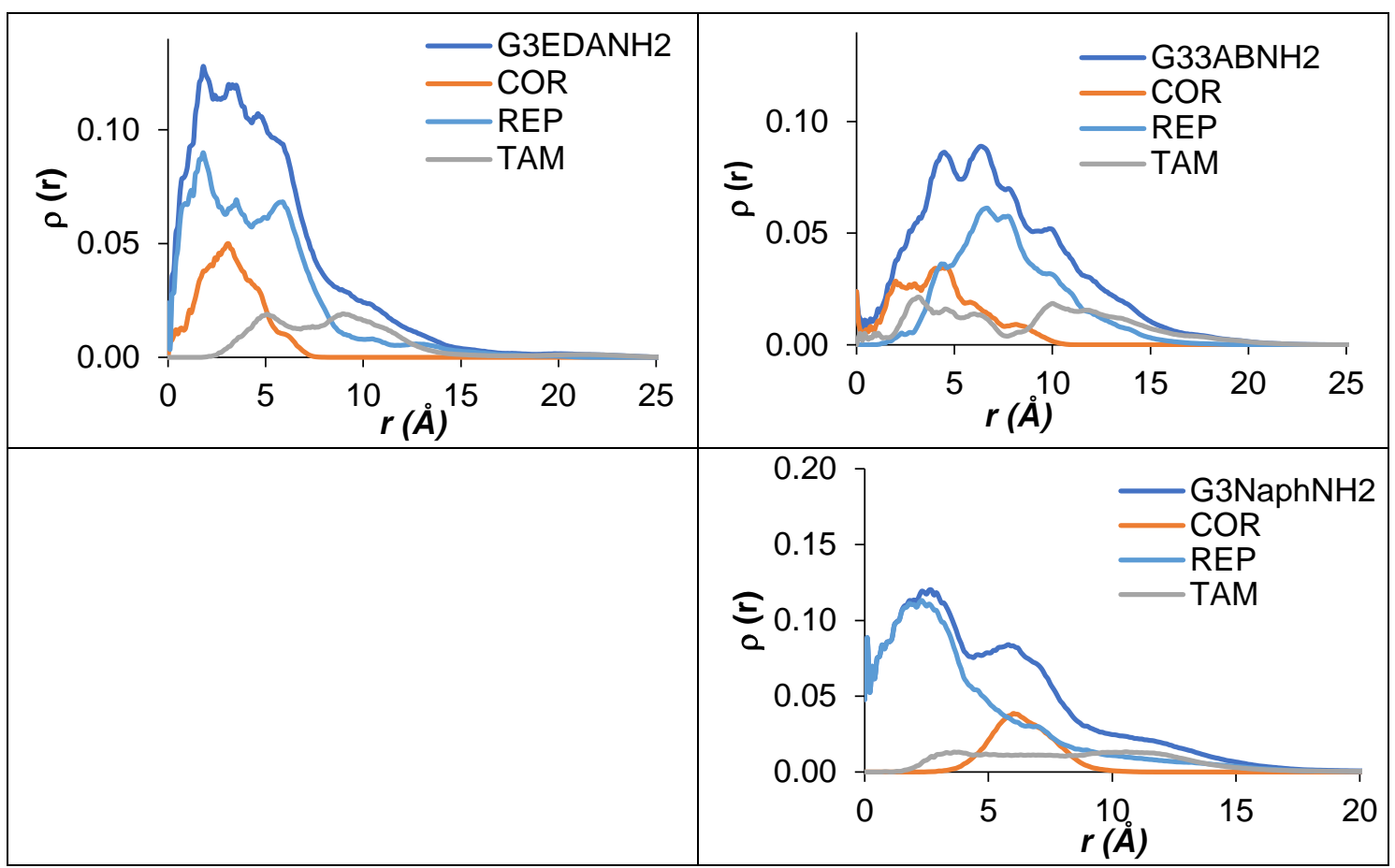

Figure S87. Radial distribution function of the $\mathbf{G 3}-\mathbf{N H}_{2}$ dendrimers and its monomers using dendrimer center of mass as reference. The unit value for $\rho(r)$ is expressed in atoms $/ \AA^{3}$.

\section{Computed total energies and starting geometries of optimized structures}

\begin{tabular}{lccc}
\hline Dynamics summary & Final & $\begin{array}{c}\text { Average } \\
\text { (the last 1 ns) }\end{array}$ & $\begin{array}{c}\text { Std. Dev. } \\
\text { (the last 1 ns) }\end{array}$ \\
\hline Tot. energy (kcal/mol) & -21215.853 & -21225.835 & 16.899 \\
Pot. energy (kcal/mol) & -26055.230 & -26055.370 & 45.273 \\
Kin. energy $(\mathrm{kcal} / \mathrm{mol})$ & 4839.378 & 4829.535 & 41.065 \\
Temperature $(\mathrm{K})$ & 300.520 & 299.911 & 2.550 \\
\hline
\end{tabular}

Table S2. Dynamics summary of dendrimer G1.EDANH2
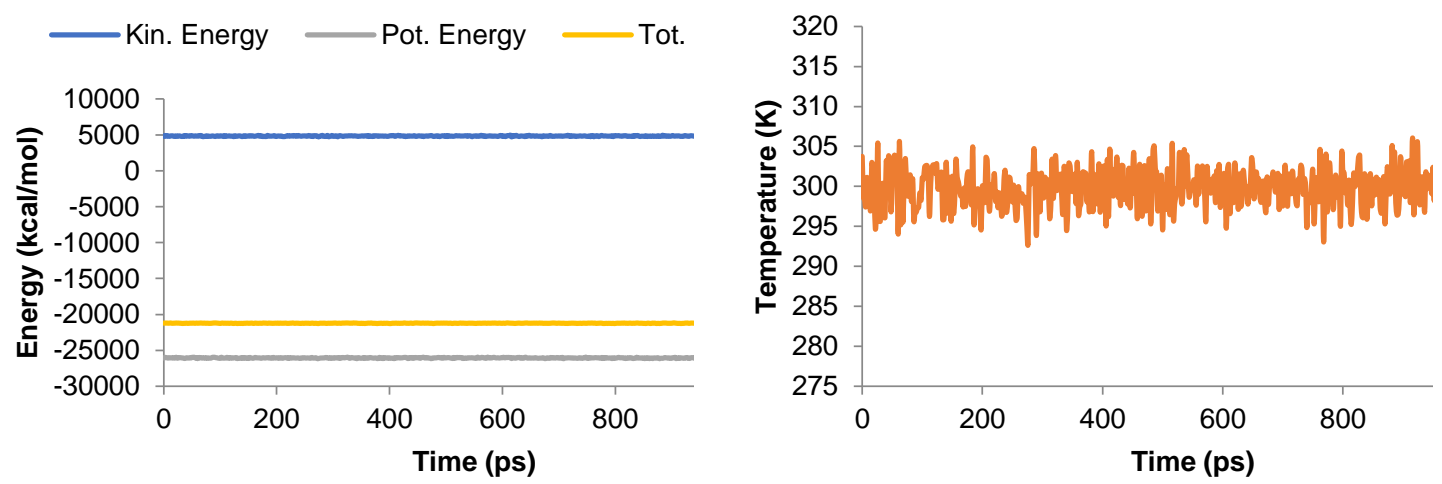

Figure S88: The energy and temperature profiles (last $1 \mathrm{~ns}$ ) of the MD simulation on dendrimer $\mathbf{G 1}_{\mathbf{E D A N H}} \mathbf{N H}_{2}$. 


\begin{tabular}{lccc}
\hline Dynamics summary & Final & $\begin{array}{c}\text { Average } \\
\text { (the last 1 ns) }\end{array}$ & $\begin{array}{c}\text { Std. Dev. } \\
\text { (the last 1 ns) }\end{array}$ \\
\hline Tot. energy (kcal/mol) & -34033.406 & -34030.136 & 22.344 \\
Pot. energy (kcal/mol) & -41675.681 & -41772.793 & 60.425 \\
Kin. energy (kcal/mol) & 7642.276 & 7742.657 & 56.674 \\
Temperature (K) & 296.000 & 299.887 & 2.195 \\
\hline \multicolumn{2}{l}{ Table S3. Dynamics summary of dendrimer G2 $\mathbf{E D D A N H}_{2}$}
\end{tabular}
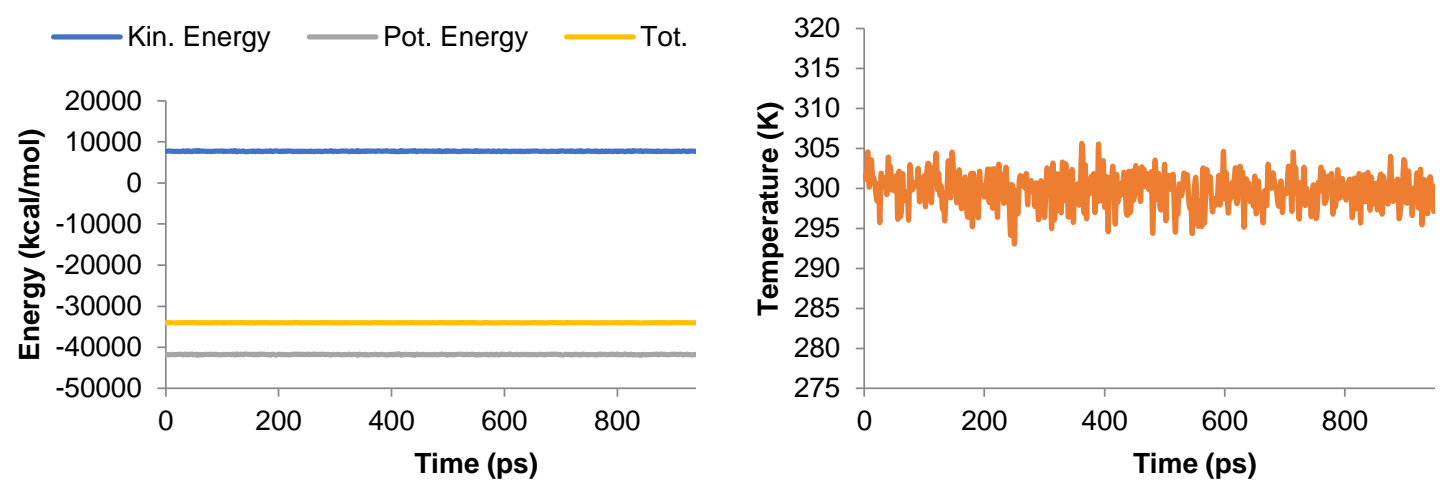

Figure S89: The energy and temperature profiles (last $1 \mathrm{~ns}$ ) of the MD simulation on dendrimer G2 $\mathbf{E D A N H}$.

\begin{tabular}{lccc}
\hline Dynamics summary & Final & $\begin{array}{c}\text { Average } \\
\text { (the last 1 ns) }\end{array}$ & $\begin{array}{c}\text { Std. Dev. } \\
\text { (the last 1 ns) }\end{array}$ \\
\hline Tot. energy $(\mathrm{kcal} / \mathrm{mol})$ & -44700.769 & -44725.356 & 27.014 \\
Pot. energy $(\mathrm{kcal} / \mathrm{mol})$ & -54789.332 & -54863.240 & 65.206 \\
Kin. energy $(\mathrm{kcal} / \mathrm{mol})$ & 10088.563 & 10137.883 & 58.429 \\
Temperature $(\mathrm{K})$ & 298.500 & 299.963 & 1.729 \\
\hline Table S4. Dynamics summary of dendrimer $\mathbf{G 3} \mathbf{B D A N H}_{\mathbf{E}}$
\end{tabular}

Table S4. Dynamics summary of dendrimer $\mathbf{G 3}_{\mathbf{E D A}} \mathbf{N H}_{2}$
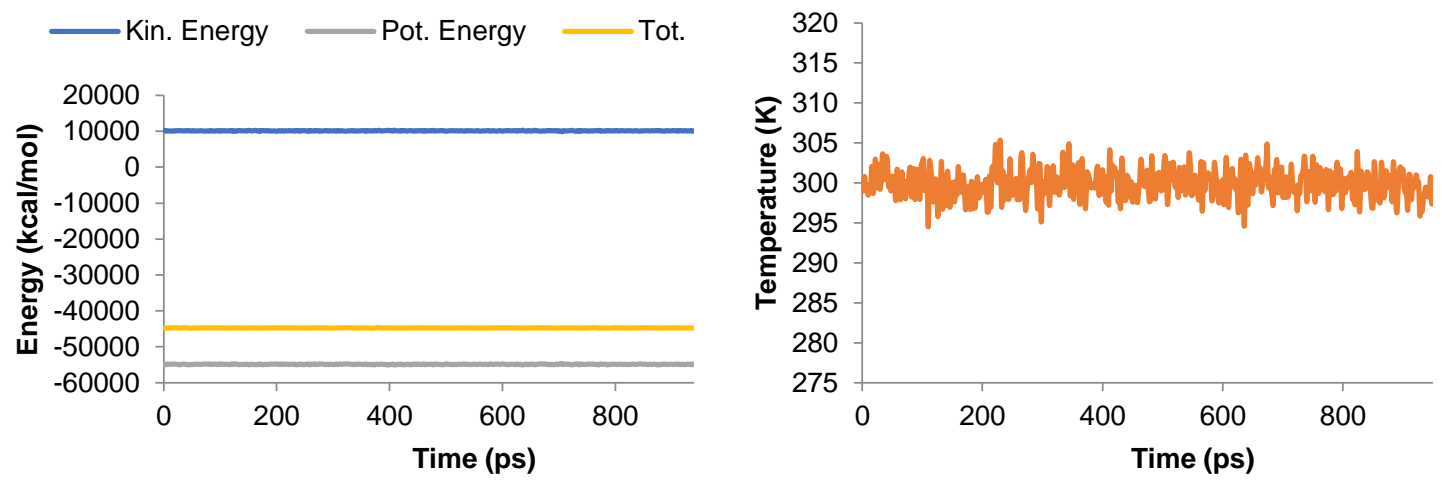

Figure S90: The energy and temperature profiles (last $1 \mathrm{~ns}$ ) of the MD simulation on dendrimer $\mathbf{G 3}_{\mathbf{E D A}} \mathbf{N H}_{2}$. 


\begin{tabular}{lccc}
\hline Dynamics summary & Final & $\begin{array}{c}\text { Average } \\
\text { (the last 1 ns) }\end{array}$ & $\begin{array}{c}\text { Std. Dev. } \\
\text { (the last 1 ns) }\end{array}$ \\
\hline Tot. energy (kcal/mol) & -49933.068 & -49979.860 & 28.569 \\
Pot. energy (kcal/mol) & -61122.640 & -61245.925 & 69.975 \\
Kin. energy (kcal/mol) & 11189.572 & 11266.065 & 62.327 \\
Temperature (K) & 297.720 & 299.751 & 1.658 \\
\hline
\end{tabular}

Table S5. Dynamics summary of dendrimer $\mathbf{G 3}_{3 \mathbf{3}} \mathbf{N H}_{2}$
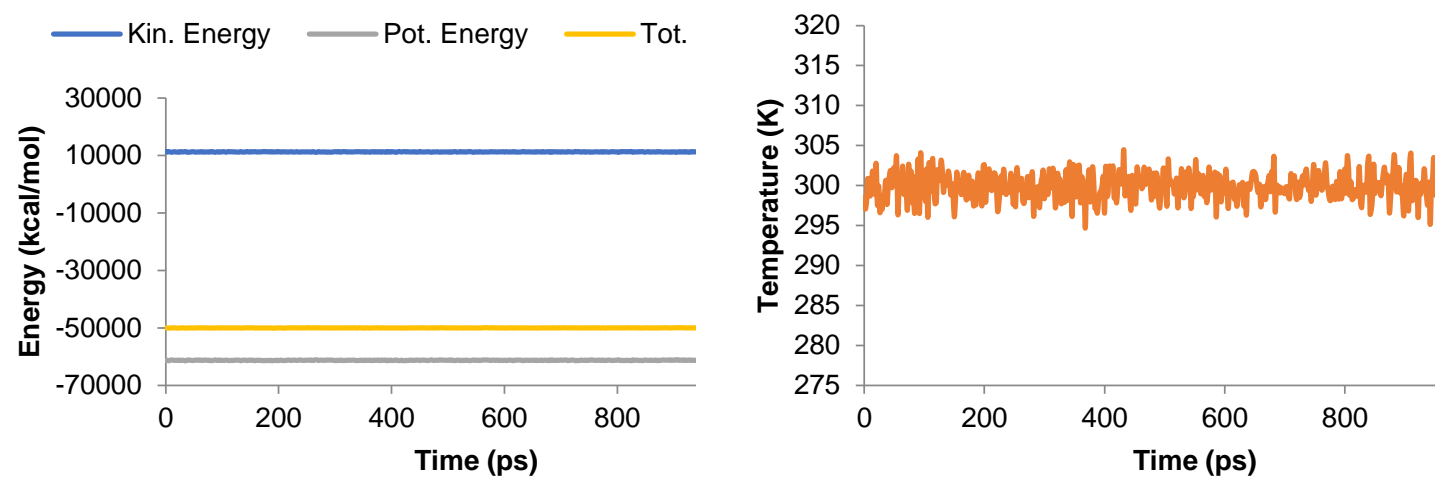

Figure S91: The energy and temperature profiles (last $1 \mathrm{~ns}$ ) of the MD simulation on dendrimer $\mathbf{G} \mathbf{3}_{\mathbf{3}} \mathbf{A B} \mathbf{N H}_{2}$.

\begin{tabular}{lccc}
\hline Dynamics summary & Final & $\begin{array}{c}\text { Average } \\
\text { (the last 1 ns) }\end{array}$ & $\begin{array}{c}\text { Std. Dev. } \\
\text { (the last 1 ns) }\end{array}$ \\
\hline Tot. energy (kcal/mol) & -66695.202 & -66711.751 & 29.229 \\
Pot. energy (kcal/mol) & -81879.841 & -81954.817 & 81.804 \\
Kin. energy $(\mathrm{kcal} / \mathrm{mol})$ & 15184.639 & 15243.066 & 73.928 \\
Temperature $(\mathrm{K})$ & 298.710 & 299.863 & 1.454 \\
\hline
\end{tabular}

Table S6. Dynamics summary of dendrimer $\mathbf{G 3}_{\mathbf{N a p h}} \mathbf{N H}_{2}$
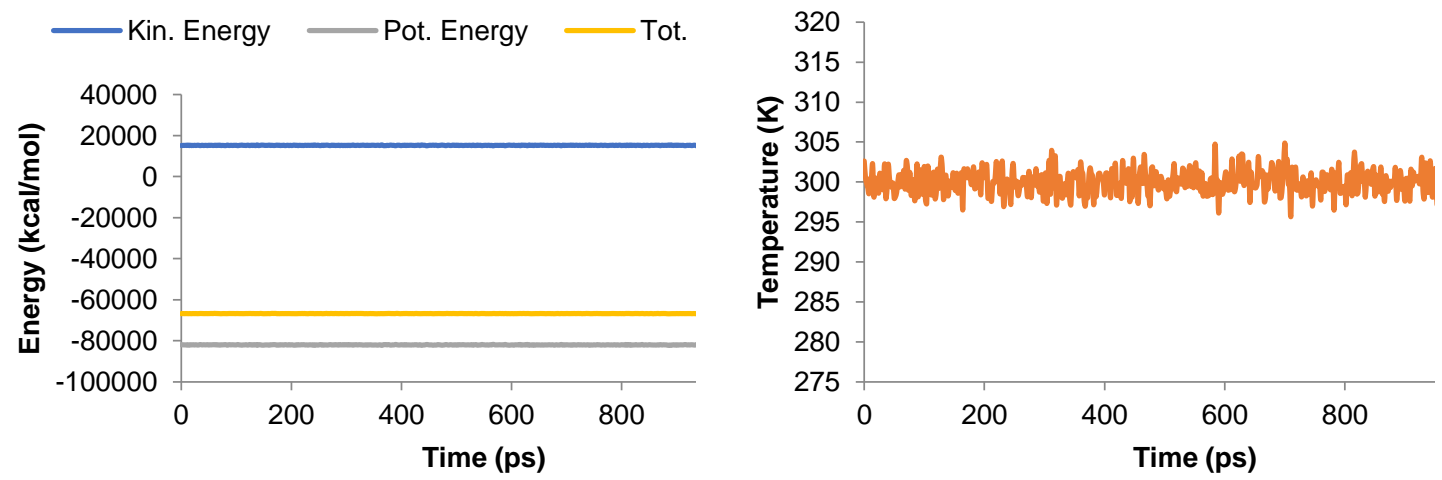

Figure S92: The energy and temperature profiles (last $1 \mathrm{~ns}$ ) of the MD simulation on dendrimer $\mathbf{G} \mathbf{3}_{\mathbf{N a p h}} \mathbf{N H}_{2}$. 
Atomic coordinates for the optimized ground state $\left(\mathrm{S}_{0}\right)$ of cap-COR (GnEDANH2) at B3LYP/6-31G(d)

\begin{tabular}{|c|c|c|c|}
\hline Energy (Hartrees) & \multicolumn{3}{|c|}{-1056.5413434} \\
\hline Imaginay freq. & 0 & & \\
\hline & \multicolumn{3}{|c|}{ Coordinates (Angstroms) } \\
\hline Atomic type & $\mathrm{X}$ & $\mathrm{Y}$ & $\mathrm{X}$ \\
\hline $\mathrm{C}$ & -0.245214 & 0.290881 & -0.668320 \\
\hline $\mathrm{H}$ & -0.331769 & -0.502960 & -1.412618 \\
\hline $\mathrm{H}$ & 0.461622 & 1.040314 & -1.034796 \\
\hline $\mathrm{C}$ & 0.245214 & -0.290881 & 0.668320 \\
\hline $\mathrm{H}$ & -0.461622 & -1.040314 & 1.034796 \\
\hline $\mathrm{H}$ & 0.331769 & 0.502960 & 1.412618 \\
\hline $\mathrm{C}$ & 2.766176 & -0.506255 & 0.933177 \\
\hline $\mathrm{C}$ & 3.633767 & -1.475812 & 0.472126 \\
\hline $\mathrm{H}$ & 2.934190 & 0.401216 & 1.492034 \\
\hline $\mathrm{C}$ & -2.766176 & 0.506255 & -0.933177 \\
\hline $\mathrm{C}$ & -3.633767 & 1.475812 & -0.472126 \\
\hline $\mathrm{H}$ & -2.934190 & -0.401216 & -1.492034 \\
\hline $\mathrm{N}$ & 1.545572 & -0.922854 & 0.512630 \\
\hline $\mathrm{N}$ & 1.644237 & -2.085360 & -0.177948 \\
\hline $\mathrm{N}$ & 2.906842 & -2.416357 & -0.200738 \\
\hline $\mathrm{N}$ & -1.545572 & 0.922854 & -0.512630 \\
\hline $\mathrm{N}$ & -1.644237 & 2.085360 & 0.177948 \\
\hline $\mathrm{N}$ & -2.906842 & 2.416357 & 0.200738 \\
\hline $\mathrm{C}$ & 5.124460 & -1.578267 & 0.620800 \\
\hline $\mathrm{H}$ & 5.414267 & -1.399362 & 1.659461 \\
\hline $\mathrm{H}$ & 5.432716 & -2.589551 & 0.344136 \\
\hline $\mathrm{C}$ & -5.124460 & 1.578267 & -0.620800 \\
\hline $\mathrm{H}$ & -5.414267 & 1.399362 & -1.659461 \\
\hline $\mathrm{H}$ & -5.432716 & 2.589551 & -0.344136 \\
\hline $\mathrm{N}$ & 5.876604 & -0.632457 & -0.203950 \\
\hline $\mathrm{C}$ & 5.966625 & 0.684495 & 0.147274 \\
\hline $\mathrm{H}$ & 6.131529 & -0.912816 & -1.140146 \\
\hline $\mathrm{O}$ & 5.492809 & 1.113534 & 1.195926 \\
\hline $\mathrm{N}$ & -5.876604 & 0.632457 & 0.203950 \\
\hline $\mathrm{C}$ & -5.966625 & -0.684495 & -0.147274 \\
\hline $\mathrm{H}$ & -6.131529 & 0.912816 & 1.140146 \\
\hline $\mathrm{O}$ & -5.492809 & -1.113534 & -1.195926 \\
\hline $\mathrm{C}$ & 6.703522 & 1.584044 & -0.832703 \\
\hline $\mathrm{H}$ & 7.154553 & 1.045567 & -1.671947 \\
\hline $\mathrm{H}$ & 6.005835 & 2.331918 & -1.223730 \\
\hline $\mathrm{H}$ & 7.487497 & 2.121894 & -0.291854 \\
\hline $\mathrm{C}$ & -6.703522 & -1.584044 & 0.832703 \\
\hline $\mathrm{H}$ & -6.005835 & -2.331918 & 1.223730 \\
\hline $\mathrm{H}$ & -7.154553 & -1.045567 & 1.671947 \\
\hline $\mathrm{H}$ & -7.487497 & -2.121894 & 0.291854 \\
\hline
\end{tabular}


Atomic coordinates for the optimized ground state $\left(\mathrm{S}_{0}\right)$ of cap-COR $\left(\mathbf{G 3 3}_{3} \mathbf{A B N H}_{2}\right)$ at B3LYP/6-31G(d)

\begin{tabular}{|c|c|c|c|}
\hline Energy (Hartrees) & \multicolumn{3}{|c|}{-1815.2681849} \\
\hline Imaginay freq. & \multicolumn{3}{|l|}{0} \\
\hline & \multicolumn{3}{|c|}{ Coordinates (Angstroms) } \\
\hline Atomic type & $\mathrm{X}$ & $\mathrm{Y}$ & $\mathrm{Z}$ \\
\hline $\mathrm{C}$ & -1.069209 & -1.497401 & -0.259258 \\
\hline $\mathrm{C}$ & -0.204052 & -1.224535 & 0.811297 \\
\hline $\mathrm{C}$ & 0.000243 & 0.094963 & 1.206748 \\
\hline $\mathrm{C}$ & -0.646211 & 1.147970 & 0.541817 \\
\hline $\mathrm{C}$ & -1.496208 & 0.863964 & -0.524010 \\
\hline $\mathrm{C}$ & -1.715609 & -0.461615 & -0.928967 \\
\hline $\mathrm{H}$ & -1.221739 & -2.529157 & -0.568073 \\
\hline $\mathrm{H}$ & 0.669191 & 0.320971 & 2.033982 \\
\hline $\mathrm{H}$ & -2.004943 & 1.669166 & -1.049406 \\
\hline $\mathrm{C}$ & -0.437285 & 2.581849 & 0.995980 \\
\hline $\mathrm{H}$ & -0.898076 & 2.753606 & 1.973539 \\
\hline $\mathrm{H}$ & -0.886659 & 3.280001 & 0.284141 \\
\hline $\mathrm{C}$ & 0.479821 & -2.363357 & 1.545946 \\
\hline $\mathrm{H}$ & 1.146583 & -1.976088 & 2.321370 \\
\hline $\mathrm{H}$ & -0.254433 & -3.017388 & 2.025230 \\
\hline $\mathrm{C}$ & -2.631087 & -0.754591 & -2.103836 \\
\hline $\mathrm{H}$ & -2.252727 & -0.292061 & -3.019983 \\
\hline $\mathrm{H}$ & -2.706892 & -1.831771 & -2.274565 \\
\hline $\mathrm{C}$ & 1.896197 & 3.253357 & 0.229600 \\
\hline $\mathrm{C}$ & 3.066915 & 3.402342 & 0.945848 \\
\hline $\mathrm{H}$ & 1.678313 & 3.368066 & -0.820746 \\
\hline $\mathrm{C}$ & 2.489873 & -3.044030 & 0.138171 \\
\hline $\mathrm{C}$ & 2.681399 & -4.137856 & -0.682022 \\
\hline $\mathrm{H}$ & 3.126662 & -2.207031 & 0.378640 \\
\hline $\mathrm{C}$ & -5.030404 & -0.754106 & -1.251562 \\
\hline $\mathrm{C}$ & -6.017117 & 0.206963 & -1.346484 \\
\hline $\mathrm{H}$ & -5.010097 & -1.717171 & -0.765837 \\
\hline $\mathrm{N}$ & 0.968852 & 2.934514 & 1.165070 \\
\hline $\mathrm{N}$ & 1.530361 & 2.875411 & 2.398331 \\
\hline $\mathrm{N}$ & 2.796905 & 3.162825 & 2.263026 \\
\hline $\mathrm{N}$ & 1.255228 & -3.232011 & 0.664251 \\
\hline $\mathrm{N}$ & 0.698756 & -4.373999 & 0.190273 \\
\hline $\mathrm{N}$ & 1.564646 & -4.921453 & -0.619519 \\
\hline $\mathrm{N}$ & -3.980762 & -0.224362 & -1.925014 \\
\hline $\mathrm{N}$ & -4.289235 & 1.003801 & -2.408348 \\
\hline $\mathrm{N}$ & -5.520468 & 1.261982 & -2.057087 \\
\hline $\mathrm{C}$ & 4.439302 & 3.782023 & 0.469965 \\
\hline $\mathrm{H}$ & 4.696346 & 3.220465 & -0.431583 \\
\hline $\mathrm{H}$ & 5.161703 & 3.533458 & 1.251412 \\
\hline $\mathrm{C}$ & 3.872245 & -4.513296 & -1.515763 \\
\hline $\mathrm{H}$ & 4.230238 & -3.646867 & -2.077446 \\
\hline $\mathrm{H}$ & 3.571706 & -5.288083 & -2.225603 \\
\hline $\mathrm{C}$ & -7.415114 & 0.202648 & -0.798706 \\
\hline $\mathrm{H}$ & -7.902199 & -0.752753 & -1.009375 \\
\hline $\mathrm{H}$ & -7.984180 & 0.997687 & -1.287019 \\
\hline $\mathrm{N}$ & 5.006392 & -5.020843 & -0.743165 \\
\hline
\end{tabular}




\begin{tabular}{|c|c|c|c|}
\hline $\mathrm{C}$ & 5.815453 & -4.168313 & -0.046272 \\
\hline $\mathrm{H}$ & 5.031831 & -6.007936 & -0.530047 \\
\hline $\mathrm{O}$ & 5.688071 & -2.948645 & -0.109652 \\
\hline $\mathrm{N}$ & -7.490914 & 0.412791 & 0.647525 \\
\hline $\mathrm{C}$ & -7.187911 & -0.595526 & 1.518030 \\
\hline $\mathrm{H}$ & -7.531882 & 1.362588 & 0.988650 \\
\hline $\mathrm{O}$ & -6.928477 & -1.732965 & 1.135124 \\
\hline $\mathrm{C}$ & 4.588412 & 5.201782 & 0.149184 \\
\hline $\mathrm{H}$ & 4.086205 & 5.712495 & -1.014235 \\
\hline $\mathrm{O}$ & 4.843528 & 5.833470 & 0.894921 \\
\hline $\mathrm{C}$ & 3.572137 & 5.000042 & -1.872110 \\
\hline $\mathrm{H}$ & 6.896168 & -4.823106 & 0.800083 \\
\hline $\mathrm{H}$ & 6.715378 & -4.585402 & 1.853522 \\
\hline $\mathrm{H}$ & 6.948565 & -5.910190 & 0.685039 \\
\hline $\mathrm{C}$ & 7.863160 & -4.389269 & 0.529549 \\
\hline $\mathrm{H}$ & -7.208718 & -0.227139 & 2.993552 \\
\hline $\mathrm{H}$ & -6.203001 & -0.357372 & 3.406371 \\
\hline $\mathrm{H}$ & -7.543278 & 0.796539 & 3.188596 \\
\hline $\mathrm{C}$ & 4.868977 & -0.923422 & 3.518670 \\
\hline $\mathrm{H}$ & 3.220645 & 7.217116 & -1.190928 \\
\hline $\mathrm{H}$ & 4.769074 & 7.657449 & -1.262693 \\
\hline $\mathrm{H}$ & 4.731691 & 7.709429 & -0.381842 \\
\hline & & & -2.137973 \\
\hline
\end{tabular}

Atomic coordinates for the optimized ground state $\left(\mathrm{S}_{0}\right)$ of cap-COR $\left(\mathbf{G 3}_{\mathbf{N a p h}} \mathbf{N H}_{2}\right)$ at B3LYP/6-31G(d)

\begin{tabular}{|c|c|c|c|}
\hline Energy (Hartrees) & \multicolumn{4}{l|}{-1934.6977155} \\
\hline Imaginay freq. & \multicolumn{3}{|l|}{0} \\
\hline & \multicolumn{3}{|l|}{ Coordinates (Angstroms) } \\
\hline Atomic type & $\mathrm{X}$ & $\mathrm{Y}$ & $\mathrm{X}$ \\
\hline $\mathrm{C}$ & -0.541742 & -0.276647 & 3.307243 \\
\hline $\mathrm{C}$ & -1.479482 & -0.476377 & 2.310273 \\
\hline $\mathrm{C}$ & -1.115937 & -1.015368 & 1.052211 \\
\hline $\mathrm{C}$ & 0.261196 & -1.318595 & 0.822030 \\
\hline $\mathrm{C}$ & 1.205252 & -1.107902 & 1.860016 \\
\hline $\mathrm{C}$ & 0.807254 & -0.600106 & 3.085124 \\
\hline $\mathrm{C}$ & -2.066712 & -1.259101 & -0.010120 \\
\hline $\mathrm{C}$ & 0.691101 & -1.813045 & -0.436384 \\
\hline $\mathrm{C}$ & -0.241286 & -1.997458 & -1.444333 \\
\hline $\mathrm{C}$ & -1.600396 & -1.732558 & -1.239273 \\
\hline $\mathrm{C}$ & 2.106677 & -2.127162 & -0.686786 \\
\hline $\mathrm{C}$ & 2.641042 & -1.418078 & 1.644568 \\
\hline $\mathrm{H}$ & -0.847303 & 0.141834 & 4.261453 \\
\hline $\mathrm{H}$ & -2.506377 & -0.178236 & 2.501396 \\
\hline $\mathrm{H}$ & 1.558131 & -0.449714 & 3.853628 \\
\hline $\mathrm{H}$ & -2.296713 & -1.920421 & -2.047993 \\
\hline $\mathrm{H}$ & 0.103894 & -2.372725 & -2.402445 \\
\hline $\mathrm{O}$ & 2.525661 & -2.539976 & -1.762031 \\
\hline $\mathrm{O}$ & 3.490979 & -1.238772 & 2.509882 \\
\hline $\mathrm{N}$ & 2.994910 & -1.935244 & 0.392816 \\
\hline $\mathrm{C}$ & 4.411256 & -2.260645 & 0.172364 \\
\hline & & & \\
\hline
\end{tabular}




\begin{tabular}{|c|c|c|c|}
\hline $\mathrm{H}$ & 4.808029 & -2.617589 & 1.124859 \\
\hline $\mathrm{H}$ & 4.439984 & -3.065463 & -0.563619 \\
\hline $\mathrm{C}$ & 5.212753 & -1.051200 & -0.324156 \\
\hline $\mathrm{H}$ & 5.122955 & -0.235443 & 0.401706 \\
\hline $\mathrm{H}$ & 4.803489 & -0.705528 & -1.279367 \\
\hline $\mathrm{C}$ & 7.984301 & 0.769466 & -0.327854 \\
\hline $\mathrm{C}$ & 8.584925 & 1.544136 & -1.299683 \\
\hline $\mathrm{H}$ & 7.906930 & 0.889870 & 0.741677 \\
\hline $\mathrm{N}$ & 7.483632 & -0.292247 & -1.006315 \\
\hline $\mathrm{N}$ & 7.749516 & -0.184582 & -2.330011 \\
\hline $\mathrm{N}$ & 8.419297 & 0.922981 & -2.504637 \\
\hline $\mathrm{C}$ & 9.345092 & 2.831099 & -1.166480 \\
\hline $\mathrm{H}$ & 8.832158 & 3.507979 & -0.479358 \\
\hline $\mathrm{H}$ & 9.403272 & 3.308127 & -2.148267 \\
\hline $\mathrm{N}$ & 10.709481 & 2.667154 & -0.661047 \\
\hline $\mathrm{C}$ & 10.940975 & 2.462329 & 0.670267 \\
\hline $\mathrm{H}$ & 11.430023 & 2.421023 & -1.325287 \\
\hline $\mathrm{O}$ & 10.046208 & 2.523892 & 1.508540 \\
\hline $\mathrm{C}$ & 12.384422 & 2.171731 & 1.053385 \\
\hline $\mathrm{H}$ & 13.088408 & 2.261156 & 0.220126 \\
\hline $\mathrm{H}$ & 12.681738 & 2.862852 & 1.847263 \\
\hline $\mathrm{H}$ & 12.446219 & 1.158007 & 1.463011 \\
\hline $\mathrm{N}$ & -3.401729 & -0.991126 & 0.202969 \\
\hline $\mathrm{H}$ & -3.707414 & -1.031765 & 1.165230 \\
\hline $\mathrm{C}$ & -4.436672 & -1.316976 & -0.765987 \\
\hline $\mathrm{H}$ & -4.204160 & -0.806029 & -1.708869 \\
\hline $\mathrm{H}$ & -4.458962 & -2.398383 & -0.984366 \\
\hline $\mathrm{C}$ & -5.802379 & -0.854861 & -0.254013 \\
\hline $\mathrm{H}$ & -5.782077 & 0.227194 & -0.078113 \\
\hline $\mathrm{H}$ & -6.032079 & -1.339413 & 0.703339 \\
\hline $\mathrm{C}$ & -9.007087 & 0.260106 & -1.105157 \\
\hline $\mathrm{C}$ & -10.109486 & 0.173012 & -0.279086 \\
\hline $\mathrm{H}$ & -8.741540 & 0.969446 & -1.873330 \\
\hline $\mathrm{N}$ & -8.232734 & -0.791925 & -0.741965 \\
\hline $\mathrm{N}$ & -8.814540 & -1.496318 & 0.257728 \\
\hline $\mathrm{N}$ & -9.948787 & -0.910813 & 0.535864 \\
\hline $\mathrm{C}$ & -11.322116 & 1.055016 & -0.204493 \\
\hline $\mathrm{H}$ & -12.092629 & 0.539665 & 0.374208 \\
\hline $\mathrm{H}$ & -11.712606 & 1.246802 & -1.207411 \\
\hline $\mathrm{N}$ & -11.080905 & 2.355762 & 0.420954 \\
\hline $\mathrm{C}$ & -10.429411 & 3.348620 & -0.252483 \\
\hline $\mathrm{H}$ & -11.216509 & 2.439601 & 1.418126 \\
\hline $\mathrm{O}$ & -10.078882 & 3.222106 & -1.423033 \\
\hline $\mathrm{C}$ & -10.179448 & 4.629005 & 0.529214 \\
\hline $\mathrm{H}$ & -10.604878 & 4.621486 & 1.537501 \\
\hline $\mathrm{H}$ & -9.100091 & 4.798253 & 0.599336 \\
\hline $\mathrm{H}$ & -10.604475 & 5.468173 & -0.029320 \\
\hline C & 6.690542 & -1.413011 & -0.513908 \\
\hline $\mathrm{H}$ & 6.805534 & -2.209229 & -1.254161 \\
\hline $\mathrm{H}$ & 7.134069 & -1.753866 & 0.428046 \\
\hline $\mathrm{C}$ & -6.924777 & -1.196804 & -1.240881 \\
\hline $\mathrm{H}$ & -6.771892 & -0.697535 & -2.202320 \\
\hline $\mathrm{H}$ & -6.962974 & -2.275369 & -1.421733 \\
\hline
\end{tabular}


Atomic coordinates for the optimized ground state $\left(\mathrm{S}_{0}\right)$ of cap-REP at B3LYP/6-31G(d)

\begin{tabular}{|c|c|c|c|}
\hline Energy (Hartrees) & \multicolumn{3}{|c|}{-1343.1706279} \\
\hline Imaginay freq. & 0 & & \\
\hline & \multicolumn{3}{|c|}{ Coordinates (Angstroms) } \\
\hline Atomic type & $\mathrm{X}$ & $\mathrm{Y}$ & $\mathrm{X}$ \\
\hline $\mathrm{C}$ & 0.165659 & 1.878515 & 0.356597 \\
\hline $\mathrm{O}$ & 0.942552 & 1.988608 & 1.298017 \\
\hline $\mathrm{N}$ & -0.476756 & 2.946640 & -0.193734 \\
\hline $\mathrm{H}$ & -1.357060 & 2.794347 & -0.676440 \\
\hline $\mathrm{C}$ & -0.293912 & 4.261766 & 0.409360 \\
\hline $\mathrm{H}$ & 0.773834 & 4.469035 & 0.502706 \\
\hline $\mathrm{H}$ & -0.755276 & 5.009689 & -0.239819 \\
\hline $\mathrm{H}$ & -0.742661 & 4.322963 & 1.408871 \\
\hline $\mathrm{C}$ & -0.002723 & 0.487223 & -0.322008 \\
\hline $\mathrm{C}$ & -1.237767 & 0.325289 & -1.249431 \\
\hline $\mathrm{H}$ & -1.203484 & 1.042899 & -2.073595 \\
\hline $\mathrm{H}$ & -1.218207 & -0.677635 & -1.688854 \\
\hline $\mathrm{C}$ & -0.001495 & -0.588155 & 0.776606 \\
\hline $\mathrm{H}$ & 0.008428 & -1.595695 & 0.341510 \\
\hline $\mathrm{H}$ & 0.870314 & -0.462855 & 1.419403 \\
\hline $\mathrm{H}$ & -0.887214 & -0.497794 & 1.413045 \\
\hline $\mathrm{C}$ & 1.203273 & 0.290402 & -1.300956 \\
\hline $\mathrm{H}$ & 1.154363 & -0.720489 & -1.722992 \\
\hline $\mathrm{H}$ & 1.131997 & 1.010789 & -2.120073 \\
\hline $\mathrm{C}$ & -3.322047 & -0.380003 & 0.051086 \\
\hline $\mathrm{C}$ & -4.450487 & 0.343113 & 0.386638 \\
\hline $\mathrm{H}$ & -3.059922 & -1.412271 & 0.219563 \\
\hline $\mathrm{C}$ & 3.281383 & -0.335320 & 0.032778 \\
\hline $\mathrm{C}$ & 4.434919 & 0.381823 & 0.266810 \\
\hline $\mathrm{H}$ & 2.985799 & -1.328888 & 0.328867 \\
\hline $\mathrm{N}$ & -2.544626 & 0.499604 & -0.625975 \\
\hline $\mathrm{N}$ & -3.162629 & 1.704173 & -0.714446 \\
\hline $\mathrm{N}$ & -4.308593 & 1.609895 & -0.099918 \\
\hline $\mathrm{N}$ & 2.526370 & 0.484129 & -0.740797 \\
\hline $\mathrm{N}$ & 3.179626 & 1.650487 & -0.980916 \\
\hline $\mathrm{N}$ & 4.329655 & 1.584354 & -0.374005 \\
\hline $\mathrm{C}$ & -5.680517 & -0.094552 & 1.128787 \\
\hline $\mathrm{H}$ & -5.399384 & -0.655244 & 2.024032 \\
\hline $\mathrm{H}$ & -6.237523 & 0.793646 & 1.436591 \\
\hline $\mathrm{C}$ & 5.660368 & -0.004756 & 1.041261 \\
\hline $\mathrm{H}$ & 6.221088 & 0.900380 & 1.287461 \\
\hline $\mathrm{H}$ & 5.379059 & -0.502570 & 1.972293 \\
\hline $\mathrm{N}$ & -6.578258 & -0.945119 & 0.347747 \\
\hline $\mathrm{C}$ & -6.272831 & -2.255420 & 0.113105 \\
\hline $\mathrm{H}$ & -7.313505 & -0.503307 & -0.185192 \\
\hline $\mathrm{O}$ & -5.277245 & -2.788148 & 0.596636 \\
\hline $\mathrm{N}$ & 6.559837 & -0.910708 & 0.323227 \\
\hline $\mathrm{C}$ & 6.258409 & -2.235745 & 0.188536 \\
\hline $\mathrm{H}$ & 7.256341 & -0.502021 & -0.283671 \\
\hline $\mathrm{O}$ & 5.287885 & -2.747029 & 0.740785 \\
\hline $\mathrm{C}$ & -7.248478 & -3.018995 & -0.768001 \\
\hline
\end{tabular}




\begin{tabular}{|c|c|c|c|}
\hline $\mathrm{H}$ & -6.723401 & -3.363021 & -1.664908 \\
\hline $\mathrm{H}$ & -7.585485 & -3.908044 & -0.226942 \\
\hline $\mathrm{H}$ & -8.122575 & -2.433737 & -1.069779 \\
\hline $\mathrm{C}$ & 7.210866 & -3.046995 & -0.677302 \\
\hline $\mathrm{H}$ & 7.534133 & -3.926019 & -0.112485 \\
\hline $\mathrm{H}$ & 6.671203 & -3.406219 & -1.559941 \\
\hline $\mathrm{H}$ & 8.093509 & -2.488702 & -1.004636 \\
\hline
\end{tabular}

Atomic coordinates for the optimized ground state $\left(\mathrm{S}_{0}\right)$ of cap-TAM at $\operatorname{PCM}\left(\mathrm{H}_{2} \mathrm{O}\right) / \mathrm{B} 3 \mathrm{LYP} / 6-31 \mathrm{G}(\mathrm{d})$

\begin{tabular}{|c|c|c|c|}
\hline Energy (Hartrees) & \multicolumn{4}{|l|}{-477.7727134} \\
\hline Imaginay freq. & \multicolumn{3}{|l|}{0} \\
\hline & \multicolumn{2}{|c|}{ Coordinates (Angstroms) } \\
\hline Atomic type & $\mathrm{X}$ & $\mathrm{Y}$ & $\mathrm{X}$ \\
\hline $\mathrm{C}$ & 0.674764 & -0.111720 & 0.417600 \\
\hline $\mathrm{C}$ & -0.797608 & 0.080363 & -0.011465 \\
\hline $\mathrm{C}$ & -0.976643 & 1.364466 & -0.836871 \\
\hline $\mathrm{N}$ & -0.436631 & 2.568622 & -0.125946 \\
\hline $\mathrm{C}$ & -1.212792 & -1.068615 & -0.944022 \\
\hline $\mathrm{N}$ & -0.962847 & -2.405793 & -0.311920 \\
\hline $\mathrm{C}$ & -1.688203 & 0.116522 & 1.233943 \\
\hline $\mathrm{O}$ & 0.962429 & -0.358825 & 1.596608 \\
\hline $\mathrm{N}$ & 1.609992 & -0.016307 & -0.552793 \\
\hline $\mathrm{C}$ & 3.021633 & -0.221529 & -0.237179 \\
\hline $\mathrm{H}$ & -2.035256 & 1.558167 & -1.021617 \\
\hline $\mathrm{H}$ & -0.466031 & 1.328377 & -1.799790 \\
\hline $\mathrm{H}$ & 0.575355 & 2.495431 & 0.042418 \\
\hline $\mathrm{H}$ & -0.895978 & 2.734685 & 0.777286 \\
\hline $\mathrm{H}$ & -2.280052 & -1.022233 & -1.170946 \\
\hline $\mathrm{H}$ & -0.657319 & -1.068527 & -1.883613 \\
\hline $\mathrm{H}$ & -1.497733 & -2.537903 & 0.554899 \\
\hline $\mathrm{H}$ & 0.030404 & -2.555780 & -0.093392 \\
\hline $\mathrm{H}$ & -1.583289 & -0.791623 & 1.829307 \\
\hline $\mathrm{H}$ & -1.408197 & 0.931997 & 1.903870 \\
\hline $\mathrm{H}$ & -2.735056 & 0.236487 & 0.943752 \\
\hline $\mathrm{H}$ & 1.357903 & 0.139566 & -1.521045 \\
\hline $\mathrm{H}$ & 3.592095 & -0.116070 & -1.158035 \\
\hline $\mathrm{H}$ & 3.175100 & -1.218933 & 0.178879 \\
\hline $\mathrm{H}$ & 3.355407 & 0.521356 & 0.489033 \\
\hline $\mathrm{H}$ & -0.583883 & 3.415310 & -0.691717 \\
\hline & -1.243405 & -3.162289 & -0.949568 \\
\hline & & & \\
\hline
\end{tabular}


Atomic coordinates for the optimized structure of G1EDANH2 with AMBER at MM level (parm99, gaff)

\begin{tabular}{|c|c|c|c|}
\hline Energy (kcal/mol) & \multicolumn{3}{|l|}{213.03} \\
\hline & \multicolumn{3}{|c|}{ Coordinates (Angstroms) } \\
\hline Atomic type & $\mathrm{X}$ & $\mathrm{Y}$ & $\mathrm{X}$ \\
\hline N7 & 3.947000 & 1.514000 & -0.845000 \\
\hline H11 & 4.130000 & 1.707000 & -1.814000 \\
\hline $\mathrm{C} 7$ & 2.804000 & 0.607000 & -0.573000 \\
\hline $\mathrm{H} 7$ & 2.978000 & 0.066000 & 0.361000 \\
\hline $\mathrm{H} 8$ & 2.729000 & -0.140000 & -1.367000 \\
\hline $\mathrm{C} 4$ & 1.509000 & 1.354000 & -0.474000 \\
\hline $\mathrm{C} 3$ & 0.309000 & 1.005000 & 0.111000 \\
\hline H5 & -0.013000 & 0.124000 & 0.635000 \\
\hline N3 & 1.388000 & 2.602000 & -1.001000 \\
\hline $\mathrm{N} 2$ & 0.170000 & 3.051000 & -0.770000 \\
\hline N1 & -0.486000 & 2.083000 & -0.097000 \\
\hline $\mathrm{C} 2$ & -1.868000 & 2.298000 & 0.291000 \\
\hline H3 & -2.020000 & 1.867000 & 1.281000 \\
\hline $\mathrm{H} 4$ & -2.046000 & 3.372000 & 0.361000 \\
\hline $\mathrm{C} 1$ & -2.871000 & 1.682000 & -0.704000 \\
\hline $\mathrm{H} 1$ & -2.735000 & 0.601000 & -0.739000 \\
\hline $\mathrm{H} 2$ & -2.680000 & 2.080000 & -1.702000 \\
\hline $\mathrm{N} 4$ & -4.253000 & 1.957000 & -0.356000 \\
\hline $\mathrm{C} 5$ & -5.163000 & 2.725000 & -1.004000 \\
\hline $\mathrm{H} 6$ & -4.949000 & 3.243000 & -1.922000 \\
\hline N5 & -4.781000 & 1.412000 & 0.761000 \\
\hline N6 & -6.030000 & 1.828000 & 0.838000 \\
\hline C6 & -6.299000 & 2.631000 & -0.227000 \\
\hline $\mathrm{C} 8$ & -7.654000 & 3.245000 & -0.408000 \\
\hline H9 & -7.958000 & 3.138000 & -1.453000 \\
\hline $\mathrm{H} 10$ & -7.593000 & 4.316000 & -0.201000 \\
\hline $\mathrm{N} 8$ & -8.672000 & 2.623000 & 0.475000 \\
\hline $\mathrm{H} 12$ & -8.720000 & 2.989000 & 1.410000 \\
\hline $\mathrm{C} 1$ & 4.519000 & 2.258000 & 0.122000 \\
\hline $\mathrm{C} 2$ & 5.288000 & 3.525000 & -0.263000 \\
\hline $\mathrm{C} 3$ & 6.801000 & 3.134000 & -0.266000 \\
\hline N1 & 7.299000 & 2.497000 & 1.038000 \\
\hline $\mathrm{H} 3$ & 6.632000 & 1.769000 & 1.379000 \\
\hline $\mathrm{H} 4$ & 7.375000 & 3.186000 & 1.809000 \\
\hline H16 & 8.229000 & 2.048000 & 0.939000 \\
\hline H1 & 7.432000 & 4.009000 & -0.463000 \\
\hline $\mathrm{H} 2$ & 6.989000 & 2.398000 & -1.056000 \\
\hline $\mathrm{C} 4$ & 4.833000 & 4.121000 & -1.641000 \\
\hline $\mathrm{N} 2$ & 3.386000 & 4.626000 & -1.728000 \\
\hline $\mathrm{H} 7$ & 3.199000 & 5.409000 & -1.076000 \\
\hline $\mathrm{H} 8$ & 2.681000 & 3.890000 & -1.499000 \\
\hline H17 & 3.143000 & 4.971000 & -2.677000 \\
\hline $\mathrm{H} 5$ & 5.469000 & 4.978000 & -1.893000 \\
\hline H6 & 4.961000 & 3.380000 & -2.438000 \\
\hline $\mathrm{C} 5$ & 5.060000 & 4.625000 & 0.818000 \\
\hline H9 & 3.998000 & 4.853000 & 0.925000 \\
\hline $\mathrm{H} 10$ & 5.409000 & 4.303000 & 1.801000 \\
\hline
\end{tabular}




\begin{tabular}{|c|c|c|c|}
\hline H11 & 5.590000 & 5.543000 & 0.557000 \\
\hline O1 & 4.491000 & 1.902000 & 1.275000 \\
\hline C1 & -9.299000 & 1.474000 & 0.157000 \\
\hline C 3 & -9.910000 & 0.620000 & 1.271000 \\
\hline N1 & -9.264000 & 0.893000 & 2.675000 \\
\hline H3 & -7.777000 & 0.541000 & 2.828000 \\
\hline H4 & -7.165000 & 1.034000 & 2.138000 \\
\hline H16 & -7.493000 & -0.470000 & 2.696000 \\
\hline H1 & -9.793000 & 0.786000 & 3.767000 \\
\hline H2 & -9.372000 & 1.9490000 & 3.437000 \\
\hline C4 & -11.444000 & 0.923000 & 1.267000 \\
\hline N2 & -12.130000 & 0.728000 & -0.091000 \\
\hline H7 & -12.228000 & -0.272000 & -0.343000 \\
\hline H8 & -11.566000 & 1.156000 & -0.860000 \\
\hline H17 & -13.079000 & 1.146000 & -0.128000 \\
\hline H5 & -11.970000 & 0.291000 & 1.992000 \\
\hline H6 & -11.613000 & 1.970000 & 1.547000 \\
\hline C5 & -9.700000 & -0.890000 & 0.947000 \\
\hline H9 & -10.179000 & -1.170000 & 0.006000 \\
\hline H10 & -8.640000 & -1.122000 & 0.836000 \\
\hline H11 & -10.116000 & -1.520000 & 1.736000 \\
\hline O1 & -9.447000 & 1.146000 & -0.996000 \\
\hline & & &
\end{tabular}

Atomic coordinates for the optimized structure of $\mathbf{G 2} \mathbf{E D A N H}_{2}$ with AMBER at MM level (parm99, gaff)

\begin{tabular}{|c|c|c|c|}
\hline \multirow[t]{2}{*}{ Energy $(\mathrm{kcal} / \mathrm{mol})$} & \multicolumn{3}{|l|}{382.30} \\
\hline & \multicolumn{3}{|c|}{ Coordinates (Angstroms) } \\
\hline Atomic type & $\mathrm{X}$ & $\mathrm{Y}$ & $\mathrm{X}$ \\
\hline N7 & 3.027000 & 3.735000 & -0.960000 \\
\hline H11 & 2.515000 & 4.306000 & -1.615000 \\
\hline $\mathrm{C} 7$ & 2.277000 & 3.223000 & 0.208000 \\
\hline $\mathrm{H} 7$ & 2.816000 & 3.486000 & 1.120000 \\
\hline H8 & 2.247000 & 2.133000 & 0.161000 \\
\hline $\mathrm{C} 4$ & 0.886000 & 3.758000 & 0.297000 \\
\hline $\mathrm{C} 3$ & -0.285000 & 3.176000 & -0.147000 \\
\hline H5 & -0.482000 & 2.245000 & -0.646000 \\
\hline N3 & 0.593000 & 4.964000 & 0.857000 \\
\hline $\mathrm{N} 2$ & -0.717000 & 5.154000 & 0.788000 \\
\hline N1 & -1.246000 & 4.070000 & 0.179000 \\
\hline $\mathrm{C} 2$ & -2.680000 & 4.001000 & -0.033000 \\
\hline H3 & -3.115000 & 4.961000 & 0.250000 \\
\hline $\mathrm{H} 4$ & -2.870000 & 3.851000 & -1.096000 \\
\hline $\mathrm{C} 1$ & -3.370000 & 2.888000 & 0.780000 \\
\hline H1 & -2.958000 & 2.870000 & 1.790000 \\
\hline $\mathrm{H} 2$ & -3.188000 & 1.917000 & 0.320000 \\
\hline N4 & -4.800000 & 3.104000 & 0.876000 \\
\hline $\mathrm{C} 5$ & -5.733000 & 3.117000 & -0.105000 \\
\hline H6 & -5.534000 & 2.852000 & -1.125000 \\
\hline $\mathrm{N} 5$ & -5.335000 & 3.441000 & 2.066000 \\
\hline
\end{tabular}




\begin{tabular}{|c|c|c|c|}
\hline N6 & -6.620000 & 3.672000 & 1.858000 \\
\hline C6 & -6.893000 & 3.487000 & 0.543000 \\
\hline C8 & -8.260000 & 3.698000 & -0.023000 \\
\hline $\mathrm{H} 9$ & -8.232000 & 3.491000 & -1.094000 \\
\hline $\mathrm{H} 10$ & -8.523000 & 4.751000 & 0.096000 \\
\hline N8 & -9.308000 & 2.858000 & 0.602000 \\
\hline $\mathrm{H} 12$ & -10.054000 & 3.337000 & 1.082000 \\
\hline $\mathrm{C} 1$ & 4.375000 & 3.729000 & -1.054000 \\
\hline $\mathrm{O} 1$ & 5.027000 & 3.099000 & -0.252000 \\
\hline $\mathrm{C} 3$ & 5.015000 & 4.509000 & -2.213000 \\
\hline $\mathrm{C} 4$ & 4.991000 & 3.625000 & -3.494000 \\
\hline H5 & 3.967000 & 3.321000 & -3.712000 \\
\hline H6 & 5.336000 & 4.223000 & -4.340000 \\
\hline $\mathrm{N} 2$ & 5.810000 & 2.421000 & -3.433000 \\
\hline $\mathrm{C} 7$ & 5.617000 & 1.274000 & -2.734000 \\
\hline $\mathrm{C} 8$ & 6.714000 & 0.497000 & -3.049000 \\
\hline N4 & 7.504000 & 1.191000 & -3.911000 \\
\hline N3 & 6.956000 & 2.367000 & -4.145000 \\
\hline C11 & 7.089000 & -0.879000 & -2.589000 \\
\hline $\mathrm{H} 14$ & 6.989000 & -0.929000 & -1.501000 \\
\hline $\mathrm{H} 15$ & 6.387000 & -1.604000 & -3.007000 \\
\hline N8 & 8.474000 & -1.238000 & -2.982000 \\
\hline $\mathrm{H} 18$ & 8.584000 & -1.574000 & -3.922000 \\
\hline $\mathrm{H} 12$ & 4.784000 & 1.122000 & -2.072000 \\
\hline $\mathrm{C} 5$ & 6.492000 & 4.865000 & -1.868000 \\
\hline $\mathrm{H7}$ & 6.948000 & 5.429000 & -2.683000 \\
\hline $\mathrm{H} 8$ & 6.545000 & 5.460000 & -0.957000 \\
\hline H9 & 7.086000 & 3.967000 & -1.700000 \\
\hline C6 & 4.252000 & 5.839000 & -2.520000 \\
\hline $\mathrm{H} 10$ & 4.840000 & 6.449000 & -3.208000 \\
\hline H11 & 3.318000 & 5.605000 & -3.033000 \\
\hline N5 & 3.930000 & 6.656000 & -1.357000 \\
\hline C9 & 4.744000 & 7.394000 & -0.562000 \\
\hline $\mathrm{H} 13$ & 5.793000 & 7.527000 & -0.756000 \\
\hline N6 & 2.656000 & 6.682000 & -0.908000 \\
\hline N7 & 2.647000 & 7.422000 & 0.180000 \\
\hline $\mathrm{C} 10$ & 3.907000 & 7.871000 & 0.425000 \\
\hline $\mathrm{C} 12$ & 4.213000 & 8.719000 & 1.621000 \\
\hline H16 & 5.086000 & 8.315000 & 2.139000 \\
\hline $\mathrm{H} 17$ & 4.479000 & 9.726000 & 1.285000 \\
\hline N9 & 3.065000 & 8.776000 & 2.559000 \\
\hline H19 & 2.716000 & 7.879000 & 2.850000 \\
\hline $\mathrm{C} 1$ & -9.309000 & 1.506000 & 0.589000 \\
\hline $\mathrm{O} 1$ & -8.440000 & 0.906000 & -0.008000 \\
\hline $\mathrm{C} 3$ & -10.470000 & 0.803000 & 1.303000 \\
\hline $\mathrm{C} 4$ & -10.704000 & 1.402000 & 2.724000 \\
\hline $\mathrm{H} 5$ & -10.956000 & 2.460000 & 2.655000 \\
\hline H6 & -11.562000 & 0.903000 & 3.178000 \\
\hline N2 & -9.577000 & 1.265000 & 3.634000 \\
\hline $\mathrm{C} 7$ & -8.455000 & 2.016000 & 3.735000 \\
\hline $\mathrm{C} 8$ & -7.723000 & 1.403000 & 4.729000 \\
\hline N4 & -8.431000 & 0.338000 & 5.189000 \\
\hline N3 & -9.565000 & 0.252000 & 4.525000 \\
\hline
\end{tabular}




\begin{tabular}{|c|c|c|c|}
\hline C11 & -6.394000 & 1.755000 & 5.322000 \\
\hline H14 & -5.606000 & 1.450000 & 4.626000 \\
\hline $\mathrm{H} 15$ & -6.318000 & 2.840000 & 5.425000 \\
\hline N8 & -6.201000 & 1.103000 & 6.643000 \\
\hline H18 & -6.841000 & 1.398000 & 7.357000 \\
\hline $\mathrm{H} 12$ & -8.263000 & 2.877000 & 3.124000 \\
\hline $\mathrm{C} 5$ & -10.177000 & -0.720000 & 1.450000 \\
\hline $\mathrm{H7}$ & -10.997000 & -1.215000 & 1.972000 \\
\hline $\mathrm{H} 8$ & -10.051000 & -1.195000 & 0.477000 \\
\hline H9 & -9.256000 & -0.886000 & 2.011000 \\
\hline C6 & -11.772000 & 0.995000 & 0.471000 \\
\hline $\mathrm{H} 10$ & -12.612000 & 0.573000 & 1.027000 \\
\hline H11 & -11.976000 & 2.058000 & 0.337000 \\
\hline N5 & -11.748000 & 0.374000 & -0.846000 \\
\hline C9 & -11.095000 & 0.755000 & -1.971000 \\
\hline $\mathrm{H} 13$ & -10.475000 & 1.632000 & -2.023000 \\
\hline N6 & -12.432000 & -0.773000 & -1.044000 \\
\hline N7 & -12.227000 & -1.134000 & -2.294000 \\
\hline $\mathrm{C} 10$ & -11.409000 & -0.225000 & -2.890000 \\
\hline $\mathrm{C} 12$ & -10.987000 & -0.386000 & -4.319000 \\
\hline H16 & -11.432000 & 0.412000 & -4.918000 \\
\hline $\mathrm{H} 17$ & -9.902000 & -0.266000 & -4.383000 \\
\hline N9 & -11.383000 & -1.705000 & -4.871000 \\
\hline H19 & -12.342000 & -1.782000 & -5.158000 \\
\hline $\mathrm{C} 1$ & 9.547000 & -0.870000 & -2.256000 \\
\hline $\mathrm{C} 2$ & 10.915000 & -0.780000 & -2.938000 \\
\hline $\mathrm{C} 3$ & 11.696000 & -2.070000 & -2.528000 \\
\hline N1 & 11.783000 & -2.312000 & -1.016000 \\
\hline $\mathrm{H} 3$ & 10.856000 & -2.155000 & -0.560000 \\
\hline $\mathrm{H} 4$ & 12.435000 & -1.657000 & -0.548000 \\
\hline $\mathrm{H} 16$ & 12.090000 & -3.273000 & -0.777000 \\
\hline $\mathrm{H} 1$ & 12.722000 & -2.045000 & -2.915000 \\
\hline $\mathrm{H} 2$ & 11.196000 & -2.951000 & -2.949000 \\
\hline $\mathrm{C} 4$ & 10.819000 & -0.633000 & -4.497000 \\
\hline $\mathrm{N} 2$ & 10.164000 & 0.650000 & -5.028000 \\
\hline $\mathrm{H7}$ & 10.697000 & 1.501000 & -4.772000 \\
\hline $\mathrm{H} 8$ & 9.197000 & 0.798000 & -4.659000 \\
\hline $\mathrm{H} 17$ & 10.084000 & 0.655000 & -6.063000 \\
\hline H5 & 11.827000 & -0.660000 & -4.927000 \\
\hline H6 & 10.264000 & -1.476000 & -4.924000 \\
\hline C5 & 11.684000 & 0.463000 & -2.396000 \\
\hline H9 & 11.116000 & 1.379000 & -2.572000 \\
\hline $\mathrm{H} 10$ & 11.842000 & 0.397000 & -1.318000 \\
\hline H11 & 12.660000 & 0.558000 & -2.875000 \\
\hline $\mathrm{O} 1$ & 9.462000 & -0.680000 & -1.067000 \\
\hline $\mathrm{C} 1$ & 2.344000 & 9.889000 & 2.788000 \\
\hline $\mathrm{C} 2$ & 0.915000 & 9.743000 & 3.319000 \\
\hline $\mathrm{C} 3$ & 0.330000 & 8.302000 & 3.114000 \\
\hline N1 & 0.181000 & 7.827000 & 1.666000 \\
\hline H3 & 1.071000 & 7.864000 & 1.124000 \\
\hline $\mathrm{H} 4$ & -0.517000 & 8.363000 & 1.123000 \\
\hline H16 & -0.099000 & 6.821000 & 1.596000 \\
\hline H1 & -0.671000 & 8.243000 & 3.558000 \\
\hline
\end{tabular}




\begin{tabular}{|c|c|c|c|}
\hline $\mathrm{H} 2$ & 0.954000 & 7.561000 & 3.625000 \\
\hline $\mathrm{C} 4$ & 0.964000 & 10.132000 & 4.832000 \\
\hline $\mathrm{N} 2$ & 1.578000 & 11.507000 & 5.120000 \\
\hline $\mathrm{H} 7$ & 0.955000 & 12.284000 & 4.833000 \\
\hline H8 & 2.459000 & 11.640000 & 4.575000 \\
\hline $\mathrm{H} 17$ & 1.804000 & 11.647000 & 6.123000 \\
\hline H5 & -0.040000 & 10.124000 & 5.271000 \\
\hline H6 & 1.578000 & 9.405000 & 5.376000 \\
\hline C5 & -0.021000 & 10.741000 & 2.575000 \\
\hline H9 & 0.298000 & 11.775000 & 2.721000 \\
\hline $\mathrm{H} 10$ & -0.010000 & 10.560000 & 1.499000 \\
\hline H11 & -1.048000 & 10.651000 & 2.934000 \\
\hline $\mathrm{O} 1$ & 2.824000 & 10.989000 & 2.644000 \\
\hline $\mathrm{C} 1$ & -5.526000 & -0.054000 & 6.786000 \\
\hline $\mathrm{C} 2$ & -5.918000 & -1.031000 & 7.904000 \\
\hline $\mathrm{C} 3$ & -4.736000 & -1.055000 & 8.927000 \\
\hline N1 & -3.371000 & -1.419000 & 8.335000 \\
\hline $\mathrm{H} 3$ & -3.214000 & -0.905000 & 7.439000 \\
\hline $\mathrm{H} 4$ & -3.300000 & -2.425000 & 8.099000 \\
\hline $\mathrm{H} 16$ & -2.583000 & -1.195000 & 8.970000 \\
\hline H1 & -4.936000 & -1.766000 & 9.737000 \\
\hline $\mathrm{H} 2$ & -4.621000 & -0.060000 & 9.373000 \\
\hline $\mathrm{C} 4$ & -7.259000 & -0.662000 & 8.631000 \\
\hline $\mathrm{N} 2$ & -8.558000 & -0.852000 & 7.838000 \\
\hline H7 & -8.737000 & -1.846000 & 7.604000 \\
\hline $\mathrm{H} 8$ & -8.555000 & -0.346000 & 6.924000 \\
\hline $\mathrm{H} 17$ & -9.393000 & -0.517000 & 8.357000 \\
\hline H5 & -7.365000 & -1.284000 & 9.527000 \\
\hline H6 & -7.232000 & 0.380000 & 8.967000 \\
\hline $\mathrm{C} 5$ & -6.078000 & -2.454000 & 7.288000 \\
\hline H9 & -6.835000 & -2.454000 & 6.501000 \\
\hline $\mathrm{H} 10$ & -5.151000 & -2.794000 & 6.823000 \\
\hline H11 & -6.362000 & -3.182000 & 8.051000 \\
\hline $\mathrm{O} 1$ & -4.585000 & -0.315000 & 6.071000 \\
\hline $\mathrm{C} 1$ & -10.625000 & -2.809000 & -4.722000 \\
\hline $\mathrm{C} 2$ & -11.285000 & -4.188000 & -4.812000 \\
\hline C3 & -12.833000 & -4.151000 & -4.555000 \\
\hline N1 & -13.288000 & -3.723000 & -3.154000 \\
\hline $\mathrm{H} 3$ & -12.934000 & -2.778000 & -2.882000 \\
\hline $\mathrm{H} 4$ & -12.960000 & -4.371000 & -2.415000 \\
\hline H16 & -14.322000 & -3.679000 & -3.067000 \\
\hline H1 & -13.251000 & -5.152000 & -4.717000 \\
\hline $\mathrm{H} 2$ & -13.321000 & -3.480000 & -5.270000 \\
\hline $\mathrm{C} 4$ & -10.954000 & -4.744000 & -6.234000 \\
\hline N2 & -9.460000 & -4.770000 & -6.578000 \\
\hline $\mathrm{H7}$ & -8.954000 & -5.526000 & -6.082000 \\
\hline $\mathrm{H} 8$ & -8.996000 & -3.880000 & -6.285000 \\
\hline H17 & -9.281000 & -4.898000 & -7.592000 \\
\hline H5 & -11.334000 & -5.766000 & -6.353000 \\
\hline H6 & -11.432000 & -4.114000 & -6.994000 \\
\hline C5 & -10.655000 & -5.133000 & -3.744000 \\
\hline $\mathrm{H} 9$ & -9.582000 & -5.257000 & -3.903000 \\
\hline $\mathrm{H} 10$ & -10.775000 & -4.722000 & -2.740000 \\
\hline
\end{tabular}




\begin{tabular}{|c|c|c|c|}
\hline H11 & -11.117000 & -6.122000 & -3.776000 \\
\hline O1 & -9.426000 & -2.727000 & -4.591000 \\
\hline
\end{tabular}

Atomic coordinates for the optimized structure of $\mathbf{G} \mathbf{3}_{\mathrm{EDANH}} \mathbf{N H}_{2}$ with AMBER at MM level (parm99, gaff)

\begin{tabular}{|c|c|c|c|}
\hline \multirow[t]{2}{*}{ Energy $(\mathrm{kcal} / \mathrm{mol})$} & \multicolumn{3}{|l|}{692.41} \\
\hline & \multicolumn{3}{|c|}{ Coordinates (Angstroms) } \\
\hline Atomic type & $X$ & $\mathrm{Y}$ & $X$ \\
\hline N7 & 3.207000 & 0.437000 & -0.629000 \\
\hline H11 & 3.610000 & 0.679000 & -1.523000 \\
\hline $\mathrm{C} 7$ & 2.354000 & -0.771000 & -0.562000 \\
\hline $\mathrm{H} 7$ & 2.705000 & -1.422000 & 0.239000 \\
\hline H8 & 2.435000 & -1.327000 & -1.499000 \\
\hline $\mathrm{C} 4$ & 0.929000 & -0.398000 & -0.328000 \\
\hline $\mathrm{C} 3$ & 0.028000 & 0.161000 & -1.204000 \\
\hline $\mathrm{H} 5$ & 0.087000 & 0.356000 & -2.257000 \\
\hline N3 & 0.357000 & -0.474000 & 0.895000 \\
\hline $\mathrm{N} 2$ & -0.863000 & 0.030000 & 0.839000 \\
\hline N1 & -1.064000 & 0.412000 & -0.439000 \\
\hline $\mathrm{C} 2$ & -2.295000 & 1.081000 & -0.798000 \\
\hline $\mathrm{H} 3$ & -3.132000 & 0.446000 & -0.504000 \\
\hline $\mathrm{H} 4$ & -2.350000 & 2.013000 & -0.235000 \\
\hline $\mathrm{C} 1$ & -2.383000 & 1.397000 & -2.301000 \\
\hline $\mathrm{H} 1$ & -2.279000 & 0.477000 & -2.877000 \\
\hline $\mathrm{H} 2$ & -1.576000 & 2.073000 & -2.580000 \\
\hline $\mathrm{N} 4$ & -3.636000 & 2.009000 & -2.660000 \\
\hline $\mathrm{C} 5$ & -4.751000 & 1.418000 & -3.135000 \\
\hline H6 & -4.877000 & 0.358000 & -3.264000 \\
\hline N5 & -3.803000 & 3.339000 & -2.522000 \\
\hline N6 & -5.041000 & 3.607000 & -2.899000 \\
\hline C6 & -5.646000 & 2.453000 & -3.274000 \\
\hline $\mathrm{C} 8$ & -7.086000 & 2.391000 & -3.657000 \\
\hline H9 & -7.250000 & 1.528000 & -4.308000 \\
\hline H10 & -7.365000 & 3.284000 & -4.218000 \\
\hline N8 & -7.946000 & 2.256000 & -2.461000 \\
\hline H12 & -8.679000 & 2.931000 & -2.323000 \\
\hline $\mathrm{C} 1$ & 3.443000 & 1.268000 & 0.409000 \\
\hline $\mathrm{O} 1$ & 3.143000 & 0.931000 & 1.541000 \\
\hline $\mathrm{C} 3$ & 3.958000 & 2.675000 & 0.085000 \\
\hline $\mathrm{C} 4$ & 4.903000 & 2.710000 & -1.155000 \\
\hline $\mathrm{H} 5$ & 4.383000 & 2.338000 & -2.038000 \\
\hline H6 & 5.169000 & 3.746000 & -1.367000 \\
\hline $\mathrm{N} 2$ & 6.147000 & 1.973000 & -1.018000 \\
\hline $\mathrm{C} 7$ & 7.356000 & 2.432000 & -0.628000 \\
\hline $\mathrm{C} 8$ & 8.183000 & 1.335000 & -0.731000 \\
\hline $\mathrm{N} 4$ & 7.456000 & 0.275000 & -1.160000 \\
\hline N3 & 6.202000 & 0.666000 & -1.338000 \\
\hline $\mathrm{C} 11$ & 9.652000 & 1.267000 & -0.479000 \\
\hline H14 & 9.897000 & 1.896000 & 0.380000 \\
\hline H15 & 9.926000 & 0.243000 & -0.223000 \\
\hline
\end{tabular}




\begin{tabular}{|c|c|c|c|}
\hline N8 & 10.443000 & 1.716000 & -1.648000 \\
\hline $\mathrm{H} 18$ & 10.967000 & 1.012000 & -2.146000 \\
\hline $\mathrm{H} 12$ & 7.557000 & 3.449000 & -0.347000 \\
\hline C5 & 4.728000 & 3.254000 & 1.306000 \\
\hline $\mathrm{H7}$ & 5.096000 & 4.257000 & 1.086000 \\
\hline H8 & 4.090000 & 3.309000 & 2.188000 \\
\hline H9 & 5.576000 & 2.619000 & 1.561000 \\
\hline C6 & 2.725000 & 3.572000 & -0.242000 \\
\hline $\mathrm{H} 10$ & 3.053000 & 4.598000 & -0.407000 \\
\hline H11 & 2.269000 & 3.223000 & -1.170000 \\
\hline N5 & 1.687000 & 3.589000 & 0.779000 \\
\hline C9 & 1.620000 & 4.333000 & 1.903000 \\
\hline H13 & 2.348000 & 5.079000 & 2.172000 \\
\hline N6 & 0.630000 & 2.755000 & 0.691000 \\
\hline N7 & -0.119000 & 2.952000 & 1.765000 \\
\hline $\mathrm{C} 10$ & 0.461000 & 3.916000 & 2.519000 \\
\hline C12 & -0.132000 & 4.395000 & 3.799000 \\
\hline H16 & -1.004000 & 3.788000 & 4.013000 \\
\hline H17 & 0.584000 & 4.277000 & 4.615000 \\
\hline N9 & -0.582000 & 5.793000 & 3.726000 \\
\hline H19 & -1.574000 & 5.938000 & 3.633000 \\
\hline $\mathrm{C} 1$ & -7.821000 & 1.257000 & -1.555000 \\
\hline $\mathrm{O} 1$ & -7.060000 & 0.335000 & -1.756000 \\
\hline C3 & -8.658000 & 1.369000 & -0.275000 \\
\hline $\mathrm{C} 4$ & -8.200000 & 2.637000 & 0.509000 \\
\hline H5 & -8.490000 & 3.528000 & -0.050000 \\
\hline H6 & -8.720000 & 2.678000 & 1.467000 \\
\hline $\mathrm{N} 2$ & -6.767000 & 2.726000 & 0.770000 \\
\hline $\mathrm{C} 7$ & -6.051000 & 2.133000 & 1.750000 \\
\hline $\mathrm{C} 8$ & -4.762000 & 2.581000 & 1.561000 \\
\hline N4 & -4.742000 & 3.417000 & 0.490000 \\
\hline N3 & -5.974000 & 3.513000 & 0.006000 \\
\hline C11 & -3.540000 & 2.245000 & 2.354000 \\
\hline H14 & -3.133000 & 1.303000 & 1.981000 \\
\hline $\mathrm{H} 15$ & -2.783000 & 3.012000 & 2.178000 \\
\hline N8 & -3.781000 & 2.109000 & 3.816000 \\
\hline H18 & -3.365000 & 2.801000 & 4.417000 \\
\hline $\mathrm{H} 12$ & -6.469000 & 1.441000 & 2.456000 \\
\hline C5 & -8.454000 & 0.114000 & 0.623000 \\
\hline H7 & -8.991000 & 0.230000 & 1.565000 \\
\hline H8 & -8.826000 & -0.781000 & 0.126000 \\
\hline H9 & -7.399000 & -0.044000 & 0.846000 \\
\hline C6 & -10.182000 & 1.479000 & -0.600000 \\
\hline $\mathrm{H} 10$ & -10.772000 & 1.099000 & 0.234000 \\
\hline H11 & -10.444000 & 2.531000 & -0.719000 \\
\hline N5 & -10.605000 & 0.787000 & -1.811000 \\
\hline C9 & -10.274000 & -0.443000 & -2.274000 \\
\hline H13 & -9.717000 & -1.169000 & -1.713000 \\
\hline N6 & -11.313000 & 1.475000 & -2.727000 \\
\hline N7 & -11.439000 & 0.696000 & -3.788000 \\
\hline C10 & -10.798000 & -0.473000 & -3.548000 \\
\hline $\mathrm{C} 12$ & -10.695000 & -1.548000 & -4.577000 \\
\hline H16 & -10.285000 & -1.115000 & -5.492000 \\
\hline
\end{tabular}




\begin{tabular}{|c|c|c|c|}
\hline H17 & -9.986000 & -2.300000 & -4.227000 \\
\hline N9 & -11.986000 & -2.203000 & -4.879000 \\
\hline H19 & -12.374000 & -2.054000 & -5.798000 \\
\hline $\mathrm{C} 1$ & 10.405000 & 2.962000 & -2.169000 \\
\hline $\mathrm{O} 1$ & 9.765000 & 3.821000 & -1.599000 \\
\hline C3 & 11.176000 & 3.241000 & -3.473000 \\
\hline $\mathrm{C} 4$ & 11.156000 & 2.028000 & -4.466000 \\
\hline H5 & 10.120000 & 1.721000 & -4.619000 \\
\hline H6 & 11.530000 & 2.359000 & -5.436000 \\
\hline $\mathrm{N} 2$ & 11.936000 & 0.844000 & -4.123000 \\
\hline C7 & 13.274000 & 0.633000 & -4.205000 \\
\hline C8 & 13.440000 & -0.667000 & -3.771000 \\
\hline N4 & 12.217000 & -1.180000 & -3.463000 \\
\hline N3 & 11.297000 & -0.263000 & -3.687000 \\
\hline C11 & 14.687000 & -1.491000 & -3.642000 \\
\hline H14 & 15.292000 & -1.377000 & -4.545000 \\
\hline H15 & 15.284000 & -1.102000 & -2.812000 \\
\hline N8 & 14.391000 & -2.928000 & -3.420000 \\
\hline H18 & 14.153000 & -3.460000 & -4.238000 \\
\hline $\mathrm{H} 12$ & 13.967000 & 1.382000 & -4.541000 \\
\hline C5 & 12.635000 & 3.635000 & -3.120000 \\
\hline $\mathrm{H7}$ & 13.200000 & 3.852000 & -4.027000 \\
\hline $\mathrm{H} 8$ & 12.651000 & 4.516000 & -2.478000 \\
\hline H9 & 13.129000 & 2.831000 & -2.575000 \\
\hline C6 & 10.482000 & 4.409000 & -4.255000 \\
\hline H10 & 10.852000 & 4.412000 & -5.282000 \\
\hline H11 & 9.410000 & 4.214000 & -4.310000 \\
\hline N5 & 10.663000 & 5.765000 & -3.747000 \\
\hline $\mathrm{C} 9$ & 10.022000 & 6.412000 & -2.742000 \\
\hline $\mathrm{H} 13$ & 9.304000 & 5.922000 & -2.110000 \\
\hline N6 & 11.545000 & 6.583000 & -4.360000 \\
\hline N7 & 11.491000 & 7.746000 & -3.741000 \\
\hline C10 & 10.570000 & 7.676000 & -2.741000 \\
\hline C12 & 10.267000 & 8.862000 & -1.877000 \\
\hline H16 & 9.186000 & 8.956000 & -1.754000 \\
\hline H17 & 10.686000 & 8.687000 & -0.882000 \\
\hline N9 & 10.818000 & 10.117000 & -2.447000 \\
\hline H19 & 10.337000 & 10.475000 & -3.251000 \\
\hline $\mathrm{C} 1$ & 0.220000 & 6.842000 & 3.448000 \\
\hline $\mathrm{O} 1$ & 1.423000 & 6.745000 & 3.568000 \\
\hline C3 & -0.476000 & 8.121000 & 2.976000 \\
\hline $\mathrm{C} 4$ & -1.769000 & 7.795000 & 2.159000 \\
\hline H5 & -2.543000 & 7.442000 & 2.843000 \\
\hline H6 & -2.148000 & 8.715000 & 1.713000 \\
\hline $\mathrm{N} 2$ & -1.632000 & 6.807000 & 1.089000 \\
\hline C7 & -0.974000 & 6.913000 & -0.086000 \\
\hline $\mathrm{C} 8$ & -1.255000 & 5.734000 & -0.741000 \\
\hline N4 & -2.064000 & 4.975000 & 0.035000 \\
\hline N3 & -2.297000 & 5.629000 & 1.162000 \\
\hline C11 & -0.980000 & 5.395000 & -2.162000 \\
\hline H14 & -0.082000 & 5.917000 & -2.506000 \\
\hline H15 & -0.809000 & 4.322000 & -2.259000 \\
\hline N8 & -2.143000 & 5.807000 & -2.982000 \\
\hline
\end{tabular}




\begin{tabular}{|c|c|c|c|}
\hline H18 & -2.746000 & 5.065000 & -3.321000 \\
\hline $\mathrm{H} 12$ & -0.399000 & 7.774000 & -0.376000 \\
\hline $\mathrm{C} 5$ & 0.483000 & 8.952000 & 2.073000 \\
\hline H7 & -0.018000 & 9.851000 & 1.712000 \\
\hline $\mathrm{H} 8$ & 1.378000 & 9.256000 & 2.618000 \\
\hline $\mathrm{H} 9$ & 0.818000 & 8.367000 & 1.218000 \\
\hline C6 & -0.884000 & 8.964000 & 4.219000 \\
\hline $\mathrm{H} 10$ & -1.522000 & 9.788000 & 3.893000 \\
\hline H11 & -1.470000 & 8.353000 & 4.905000 \\
\hline N5 & 0.230000 & 9.532000 & 4.967000 \\
\hline C9 & 1.146000 & 8.925000 & 5.760000 \\
\hline $\mathrm{H} 13$ & 1.152000 & 7.867000 & 5.948000 \\
\hline N6 & 0.469000 & 10.856000 & 4.872000 \\
\hline N7 & 1.538000 & 11.109000 & 5.600000 \\
\hline C10 & 1.983000 & 9.950000 & 6.152000 \\
\hline $\mathrm{C} 12$ & 3.185000 & 9.929000 & 7.043000 \\
\hline H16 & 3.008000 & 9.250000 & 7.880000 \\
\hline H17 & 4.034000 & 9.528000 & 6.481000 \\
\hline N9 & 3.509000 & 11.280000 & 7.564000 \\
\hline H19 & 2.807000 & 11.691000 & 8.153000 \\
\hline $\mathrm{C} 1$ & -4.354000 & 1.036000 & 4.416000 \\
\hline $\mathrm{O} 1$ & -4.827000 & 0.154000 & 3.731000 \\
\hline $\mathrm{C} 3$ & -4.442000 & 0.991000 & 5.955000 \\
\hline $\mathrm{C} 4$ & -3.544000 & 2.050000 & 6.683000 \\
\hline H5 & -3.714000 & 3.045000 & 6.271000 \\
\hline H6 & -3.835000 & 2.096000 & 7.733000 \\
\hline $\mathrm{N} 2$ & -2.117000 & 1.771000 & 6.673000 \\
\hline $\mathrm{C7}$ & -1.350000 & 1.219000 & 7.644000 \\
\hline $\mathrm{C} 8$ & -0.112000 & 1.093000 & 7.050000 \\
\hline N4 & -0.181000 & 1.574000 & 5.780000 \\
\hline N3 & -1.410000 & 1.978000 & 5.549000 \\
\hline C11 & 1.127000 & 0.410000 & 7.532000 \\
\hline H14 & 1.891000 & 1.152000 & 7.776000 \\
\hline H15 & 0.905000 & -0.152000 & 8.443000 \\
\hline N8 & 1.618000 & -0.519000 & 6.487000 \\
\hline H18 & 2.464000 & -0.249000 & 6.016000 \\
\hline H12 & -1.714000 & 0.979000 & 8.626000 \\
\hline C5 & -4.011000 & -0.427000 & 6.444000 \\
\hline $\mathrm{H} 7$ & -4.079000 & -0.492000 & 7.530000 \\
\hline $\mathrm{H} 8$ & -4.636000 & -1.209000 & 6.013000 \\
\hline H9 & -2.988000 & -0.647000 & 6.139000 \\
\hline C6 & -5.921000 & 1.289000 & 6.362000 \\
\hline $\mathrm{H} 10$ & -5.933000 & 1.740000 & 7.357000 \\
\hline H11 & -6.349000 & 2.029000 & 5.684000 \\
\hline N5 & -6.822000 & 0.144000 & 6.414000 \\
\hline C9 & -7.322000 & -0.629000 & 5.418000 \\
\hline $\mathrm{H} 13$ & -7.064000 & -0.487000 & 4.385000 \\
\hline N6 & -7.248000 & -0.283000 & 7.622000 \\
\hline N7 & -8.022000 & -1.328000 & 7.412000 \\
\hline $\mathrm{C} 10$ & -8.089000 & -1.572000 & 6.076000 \\
\hline $\mathrm{C} 12$ & -8.923000 & -2.695000 & 5.540000 \\
\hline H16 & -9.508000 & -2.340000 & 4.689000 \\
\hline H17 & -8.263000 & -3.482000 & 5.164000 \\
\hline
\end{tabular}




\begin{tabular}{|c|c|c|c|}
\hline N9 & -9.837000 & -3.250000 & 6.571000 \\
\hline H19 & -10.623000 & -2.674000 & 6.810000 \\
\hline $\mathrm{C} 1$ & -12.686000 & -2.942000 & -3.991000 \\
\hline $\mathrm{O} 1$ & -12.241000 & -3.142000 & -2.881000 \\
\hline $\mathrm{C} 3$ & -14.014000 & -3.524000 & -4.483000 \\
\hline $\mathrm{C} 4$ & -14.908000 & -2.406000 & -5.097000 \\
\hline H5 & -14.415000 & -1.957000 & -5.960000 \\
\hline H6 & -15.832000 & -2.858000 & -5.463000 \\
\hline $\mathrm{N} 2$ & -15.265000 & -1.334000 & -4.181000 \\
\hline C7 & -14.547000 & -0.255000 & -3.783000 \\
\hline $\mathrm{C} 8$ & -15.392000 & 0.414000 & -2.922000 \\
\hline N4 & -16.561001 & -0.276000 & -2.843000 \\
\hline N3 & -16.488001 & -1.341000 & -3.613000 \\
\hline C11 & -15.214000 & 1.697000 & -2.171000 \\
\hline H14 & -14.573000 & 1.512000 & -1.304000 \\
\hline $\mathrm{H} 15$ & -14.690000 & 2.416000 & -2.805000 \\
\hline N8 & -16.520000 & 2.261000 & -1.740000 \\
\hline $\mathrm{H} 18$ & -17.136999 & 2.518000 & -2.489000 \\
\hline $\mathrm{H} 12$ & -13.543000 & -0.057000 & -4.110000 \\
\hline $\mathrm{C} 5$ & -14.787000 & -4.200000 & -3.313000 \\
\hline H7 & -15.737000 & -4.600000 & -3.668000 \\
\hline $\mathrm{H} 8$ & -14.207000 & -5.017000 & -2.882000 \\
\hline $\mathrm{H} 9$ & -14.988000 & -3.482000 & -2.517000 \\
\hline C6 & -13.735000 & -4.586000 & -5.586000 \\
\hline $\mathrm{H} 10$ & -14.687000 & -4.986000 & -5.940000 \\
\hline H11 & -13.249000 & -4.115000 & -6.441000 \\
\hline N5 & -12.902000 & -5.703000 & -5.163000 \\
\hline C9 & -11.560000 & -5.756000 & -4.976000 \\
\hline $\mathrm{H} 13$ & -10.916000 & -4.904000 & -5.094000 \\
\hline N6 & -13.477000 & -6.898000 & -4.917000 \\
\hline N7 & -12.513000 & -7.725000 & -4.566000 \\
\hline $\mathrm{C} 10$ & -11.327000 & -7.061000 & -4.590000 \\
\hline $\mathrm{C} 12$ & -10.045000 & -7.763000 & -4.265000 \\
\hline H16 & -9.283000 & -7.485000 & -4.997000 \\
\hline H17 & -9.691000 & -7.420000 & -3.288000 \\
\hline N9 & -10.210000 & -9.238000 & -4.263000 \\
\hline H19 & -10.522000 & -9.631000 & -5.134000 \\
\hline $\mathrm{C} 1$ & 14.197000 & -3.444000 & -2.189000 \\
\hline $\mathrm{C} 2$ & 13.362000 & -4.719000 & -2.034000 \\
\hline C3 & 12.447000 & -5.010000 & -3.274000 \\
\hline N1 & 11.338000 & -3.989000 & -3.563000 \\
\hline $\mathrm{H} 3$ & 11.696000 & -3.012000 & -3.653000 \\
\hline $\mathrm{H} 4$ & 10.624000 & -3.956000 & -2.812000 \\
\hline H16 & 10.827000 & -4.197000 & -4.443000 \\
\hline H1 & 11.945000 & -5.975000 & -3.139000 \\
\hline $\mathrm{H} 2$ & 13.054000 & -5.088000 & -4.183000 \\
\hline $\mathrm{C} 4$ & 14.375000 & -5.881000 & -1.777000 \\
\hline $\mathrm{N} 2$ & 15.334000 & -5.646000 & -0.604000 \\
\hline H7 & 14.856000 & -5.708000 & 0.314000 \\
\hline H8 & 15.737000 & -4.682000 & -0.642000 \\
\hline H17 & 16.125000 & -6.318000 & -0.585000 \\
\hline H5 & 13.847000 & -6.823000 & -1.584000 \\
\hline H6 & 15.004000 & -6.020000 & -2.664000 \\
\hline
\end{tabular}




\begin{tabular}{|c|c|c|c|}
\hline C5 & 12.433000 & -4.583000 & -0.789000 \\
\hline $\mathrm{H} 9$ & 13.007000 & -4.435000 & 0.127000 \\
\hline $\mathrm{H} 10$ & 11.774000 & -3.719000 & -0.886000 \\
\hline H11 & 11.821000 & -5.479000 & -0.663000 \\
\hline $\mathrm{O} 1$ & 14.718000 & -2.950000 & -1.218000 \\
\hline $\mathrm{C} 1$ & 12.049000 & 10.567000 & -2.133000 \\
\hline $\mathrm{C} 2$ & 12.831000 & 11.411000 & -3.148000 \\
\hline C3 & 12.241000 & 11.358000 & -4.601000 \\
\hline N1 & 12.352000 & 10.020000 & -5.345000 \\
\hline $\mathrm{H} 3$ & 11.929000 & 9.225000 & -4.816000 \\
\hline $\mathrm{H} 4$ & 13.336000 & 9.744000 & -5.517000 \\
\hline H16 & 11.885000 & 10.039000 & -6.271000 \\
\hline H1 & 12.758000 & 12.093000 & -5.228000 \\
\hline $\mathrm{H} 2$ & 11.181000 & 11.635000 & -4.590000 \\
\hline $\mathrm{C} 4$ & 12.840000 & 12.876000 & -2.602000 \\
\hline N2 & 13.392000 & 13.028000 & -1.181000 \\
\hline $\mathrm{H} 7$ & 14.415000 & 12.874000 & -1.135000 \\
\hline $\mathrm{H} 8$ & 12.975000 & 12.309000 & -0.548000 \\
\hline H17 & 13.202000 & 13.962000 & -0.770000 \\
\hline H5 & 13.430000 & 13.533000 & -3.252000 \\
\hline H6 & 11.813000 & 13.260000 & -2.574000 \\
\hline C5 & 14.300000 & 10.894000 & -3.214000 \\
\hline $\mathrm{H} 9$ & 14.795000 & 10.967000 & -2.243000 \\
\hline H10 & 14.330000 & 9.841000 & -3.498000 \\
\hline H11 & 14.885000 & 11.469000 & -3.935000 \\
\hline O1 & 12.525000 & 10.362000 & -1.041000 \\
\hline $\mathrm{C} 1$ & -2.587000 & 7.080000 & -3.045000 \\
\hline $\mathrm{C} 2$ & -4.052000 & 7.320000 & -3.417000 \\
\hline $\mathrm{C} 3$ & -4.969000 & 7.049000 & -2.168000 \\
\hline N1 & -4.623000 & 5.907000 & -1.210000 \\
\hline $\mathrm{H} 3$ & -3.681000 & 5.964000 & -0.772000 \\
\hline $\mathrm{H} 4$ & -4.641000 & 4.970000 & -1.671000 \\
\hline H16 & -5.296000 & 5.796000 & -0.429000 \\
\hline H1 & -5.998000 & 6.875000 & -2.501000 \\
\hline $\mathrm{H} 2$ & -4.977000 & 7.946000 & -1.539000 \\
\hline $\mathrm{C} 4$ & -4.255000 & 8.826000 & -3.806000 \\
\hline $\mathrm{N} 2$ & -3.226000 & 9.437000 & -4.757000 \\
\hline H7 & -3.245000 & 9.015000 & -5.703000 \\
\hline H8 & -2.266000 & 9.305000 & -4.371000 \\
\hline $\mathrm{H} 17$ & -3.359000 & 10.459000 & -4.873000 \\
\hline H5 & -5.246000 & 8.978000 & -4.251000 \\
\hline H6 & -4.200000 & 9.436000 & -2.898000 \\
\hline C5 & -4.483000 & 6.468000 & -4.645000 \\
\hline H9 & -3.917000 & 6.758000 & -5.532000 \\
\hline $\mathrm{H} 10$ & -4.306000 & 5.409000 & -4.495000 \\
\hline H11 & -5.545000 & 6.608000 & -4.857000 \\
\hline $\mathrm{O} 1$ & -1.875000 & 8.011000 & -2.739000 \\
\hline $\mathrm{C} 1$ & 4.524000 & 12.036000 & 7.100000 \\
\hline $\mathrm{C} 2$ & 4.484000 & 13.544000 & 7.362000 \\
\hline $\mathrm{C} 3$ & 3.023000 & 14.098000 & 7.489000 \\
\hline N1 & 2.113000 & 13.912000 & 6.269000 \\
\hline $\mathrm{H} 3$ & 1.989000 & 12.909000 & 6.005000 \\
\hline $\mathrm{H} 4$ & 2.474000 & 14.391000 & 5.423000 \\
\hline
\end{tabular}




\begin{tabular}{|c|c|c|c|}
\hline H16 & 1.156000 & 14.281000 & 6.428000 \\
\hline H1 & 3.061000 & 15.177000 & 7.683000 \\
\hline $\mathrm{H} 2$ & 2.511000 & 13.635000 & 8.340000 \\
\hline $\mathrm{C} 4$ & 5.312000 & 13.788000 & 8.663000 \\
\hline $\mathrm{N} 2$ & 6.730000 & 13.207000 & 8.638000 \\
\hline H7 & 7.362000 & 13.742000 & 8.015000 \\
\hline H8 & 6.734000 & 12.231000 & 8.269000 \\
\hline H17 & 7.172000 & 13.189000 & 9.577000 \\
\hline H5 & 5.402000 & 14.861000 & 8.871000 \\
\hline H6 & 4.804000 & 13.319000 & 9.514000 \\
\hline $\mathrm{C} 5$ & 5.167000 & 14.306000 & 6.186000 \\
\hline $\mathrm{H} 9$ & 6.211000 & 14.011000 & 6.069000 \\
\hline H10 & 4.670000 & 14.089000 & 5.239000 \\
\hline H11 & 5.138000 & 15.384000 & 6.356000 \\
\hline $\mathrm{O} 1$ & 5.489000 & 11.540000 & 6.571000 \\
\hline $\mathrm{C} 1$ & 0.805000 & -1.453000 & 5.945000 \\
\hline $\mathrm{C} 2$ & 1.038000 & -1.897000 & 4.501000 \\
\hline C3 & 1.857000 & -0.850000 & 3.680000 \\
\hline N1 & 1.179000 & 0.494000 & 3.416000 \\
\hline $\mathrm{H} 3$ & 0.812000 & 0.977000 & 4.259000 \\
\hline $\mathrm{H} 4$ & 0.388000 & 0.384000 & 2.743000 \\
\hline H16 & 1.829000 & 1.130000 & 2.910000 \\
\hline $\mathrm{H} 1$ & 2.078000 & -1.254000 & 2.685000 \\
\hline $\mathrm{H} 2$ & 2.816000 & -0.638000 & 4.161000 \\
\hline $\mathrm{C} 4$ & 1.773000 & -3.270000 & 4.556000 \\
\hline $\mathrm{N} 2$ & 1.076000 & -4.328000 & 5.414000 \\
\hline H7 & 0.186000 & -4.644000 & 4.990000 \\
\hline H8 & 0.823000 & -3.942000 & 6.349000 \\
\hline H17 & 1.660000 & -5.171000 & 5.569000 \\
\hline H5 & 1.883000 & -3.686000 & 3.549000 \\
\hline H6 & 2.774000 & -3.134000 & 4.981000 \\
\hline C5 & -0.333000 & -2.091000 & 3.782000 \\
\hline H9 & -0.949000 & -2.835000 & 4.288000 \\
\hline H10 & -0.904000 & -1.162000 & 3.767000 \\
\hline H11 & -0.185000 & -2.423000 & 2.753000 \\
\hline $\mathrm{O} 1$ & -0.086000 & -1.953000 & 6.594000 \\
\hline $\mathrm{C} 1$ & -9.516000 & -4.314000 & 7.333000 \\
\hline $\mathrm{C} 2$ & -10.198000 & -4.495000 & 8.694000 \\
\hline C3 & -10.798000 & -3.165000 & 9.269000 \\
\hline N1 & -9.804000 & -2.053000 & 9.627000 \\
\hline $\mathrm{H} 3$ & -9.217000 & -1.756000 & 8.815000 \\
\hline $\mathrm{H} 4$ & -9.137000 & -2.339000 & 10.367000 \\
\hline H16 & -10.276000 & -1.196000 & 9.972000 \\
\hline $\mathrm{H} 1$ & -11.347000 & -3.387000 & 10.192000 \\
\hline $\mathrm{H} 2$ & -11.518000 & -2.737000 & 8.563000 \\
\hline $\mathrm{C} 4$ & -11.303000 & -5.580000 & 8.490000 \\
\hline $\mathrm{N} 2$ & -10.797000 & -6.905000 & 7.910000 \\
\hline H7 & -10.256000 & -7.456000 & 8.601000 \\
\hline H8 & -10.143000 & -6.735000 & 7.114000 \\
\hline H17 & -11.563000 & -7.514000 & 7.566000 \\
\hline H5 & -11.809000 & -5.810000 & 9.435000 \\
\hline H6 & -12.055000 & -5.207000 & 7.784000 \\
\hline $\mathrm{C} 5$ & -9.161000 & -5.027000 & 9.730000 \\
\hline
\end{tabular}




\begin{tabular}{|c|c|c|c|}
\hline H9 & -8.742000 & -5.987000 & 9.423000 \\
\hline $\mathrm{H} 10$ & -8.321000 & -4.338000 & 9.830000 \\
\hline H11 & -9.624000 & -5.159000 & 10.710000 \\
\hline $\mathrm{O} 1$ & -8.760000 & -5.165000 & 6.927000 \\
\hline $\mathrm{C} 1$ & -17.000000 & 2.128000 & -0.488000 \\
\hline $\mathrm{C} 2$ & -18.516001 & 2.194000 & -0.259000 \\
\hline C3 & -18.820999 & 3.630000 & 0.276000 \\
\hline N1 & -18.011000 & 4.041000 & 1.509000 \\
\hline H3 & -17.000000 & 3.813000 & 1.383000 \\
\hline $\mathrm{H} 4$ & -18.309000 & 3.531000 & 2.361000 \\
\hline $\mathrm{H} 16$ & -18.084000 & 5.055000 & 1.718000 \\
\hline $\mathrm{H} 1$ & -19.881001 & 3.734000 & 0.533000 \\
\hline $\mathrm{H} 2$ & -18.590000 & 4.368000 & -0.502000 \\
\hline $\mathrm{C} 4$ & -19.358999 & 1.882000 & -1.545000 \\
\hline $\mathrm{N} 2$ & -19.284000 & 0.452000 & -2.096000 \\
\hline $\mathrm{H7}$ & -19.641001 & -0.254000 & -1.427000 \\
\hline H8 & -18.309000 & 0.162000 & -2.333000 \\
\hline $\mathrm{H} 17$ & -19.834000 & 0.334000 & -2.968000 \\
\hline H5 & -20.420000 & 2.066000 & -1.335000 \\
\hline H6 & -19.073999 & 2.555000 & -2.361000 \\
\hline C5 & -18.923000 & 1.158000 & 0.833000 \\
\hline $\mathrm{H} 9$ & -18.629000 & 0.149000 & 0.540000 \\
\hline $\mathrm{H} 10$ & -18.427999 & 1.363000 & 1.784000 \\
\hline H11 & -20.000999 & 1.174000 & 1.003000 \\
\hline $\mathrm{O} 1$ & -16.254999 & 2.037000 & 0.460000 \\
\hline $\mathrm{C} 1$ & -10.237000 & -9.983000 & -3.143000 \\
\hline $\mathrm{C} 2$ & -10.881000 & -11.370000 & -3.207000 \\
\hline C3 & -11.978000 & -11.484000 & -4.323000 \\
\hline N1 & -13.188000 & -10.548000 & -4.204000 \\
\hline $\mathrm{H} 3$ & -12.923000 & -9.536000 & -4.220000 \\
\hline $\mathrm{H} 4$ & -13.726000 & -10.694000 & -3.331000 \\
\hline H16 & -13.861000 & -10.670000 & -4.985000 \\
\hline $\mathrm{H} 1$ & -12.382000 & -12.504000 & -4.330000 \\
\hline $\mathrm{H} 2$ & -11.537000 & -11.306000 & -5.310000 \\
\hline $\mathrm{C} 4$ & -9.716000 & -12.385000 & -3.440000 \\
\hline N2 & -8.555000 & -12.266000 & -2.445000 \\
\hline $\mathrm{H} 7$ & -8.815000 & -12.593000 & -1.497000 \\
\hline H8 & -8.259000 & -11.272000 & -2.327000 \\
\hline $\mathrm{H} 17$ & -7.719000 & -12.808000 & -2.737000 \\
\hline H5 & -10.083000 & -13.417000 & -3.392000 \\
\hline H6 & -9.283000 & -12.226000 & -4.434000 \\
\hline C5 & -11.565000 & -11.699000 & -1.846000 \\
\hline H9 & -10.845000 & -11.699000 & -1.025000 \\
\hline $\mathrm{H} 10$ & -12.323000 & -10.954000 & -1.601000 \\
\hline H11 & -12.039000 & -12.682000 & -1.877000 \\
\hline $\mathrm{O} 1$ & -9.687000 & -9.616000 & -2.132000 \\
\hline
\end{tabular}


Atomic coordinates for the optimized structure of $\mathbf{G 3 3 A B N H 2}$ with AMBER at MM level (parm99, gaff)

\begin{tabular}{|c|c|c|c|}
\hline Energy (kcal/mol) & \multicolumn{3}{|l|}{1263.30} \\
\hline & \multicolumn{3}{|c|}{ Coordinates (Angstroms) } \\
\hline Atomic type & $\mathrm{X}$ & $\mathrm{Y}$ & $\mathrm{X}$ \\
\hline $\mathrm{C} 1$ & 6.796000 & 3.262000 & 4.295000 \\
\hline C6 & 6.194000 & 4.482000 & 3.965000 \\
\hline $\mathrm{C} 5$ & 6.837000 & 5.674000 & 4.299000 \\
\hline $\mathrm{C} 4$ & 8.064000 & 5.666000 & 4.957000 \\
\hline C3 & 8.651000 & 4.443000 & 5.293000 \\
\hline $\mathrm{H} 2$ & 9.593000 & 4.434000 & 5.837000 \\
\hline $\mathrm{C} 7$ & 8.668000 & 6.986000 & 5.423000 \\
\hline $\mathrm{H} 4$ & 9.303000 & 6.833000 & 6.293000 \\
\hline H5 & 7.873000 & 7.679000 & 5.691000 \\
\hline N1 & 9.446000 & 7.628000 & 4.386000 \\
\hline $\mathrm{C} 10$ & 9.419000 & 7.475000 & 3.045000 \\
\hline $\mathrm{C} 11$ & 10.329000 & 8.397000 & 2.580000 \\
\hline N3 & 10.866000 & 9.064000 & 3.636000 \\
\hline $\mathrm{N} 2$ & 10.325000 & 8.590000 & 4.747000 \\
\hline C16 & 10.680000 & 8.699000 & 1.162000 \\
\hline H13 & 10.520000 & 7.803000 & 0.558000 \\
\hline H14 & 11.738000 & 8.954000 & 1.095000 \\
\hline N12 & 9.863000 & 9.797000 & 0.603000 \\
\hline $\mathrm{H} 21$ & 10.342000 & 10.619000 & 0.281000 \\
\hline H10 & 8.764000 & 6.800000 & 2.528000 \\
\hline $\mathrm{H} 3$ & 6.376000 & 6.629000 & 4.046000 \\
\hline C9 & 4.911000 & 4.563000 & 3.147000 \\
\hline $\mathrm{H} 8$ & 4.578000 & 5.594000 & 3.059000 \\
\hline H9 & 5.106000 & 4.198000 & 2.139000 \\
\hline N7 & 3.810000 & 3.791000 & 3.707000 \\
\hline $\mathrm{C} 14$ & 3.009000 & 2.947000 & 3.020000 \\
\hline C15 & 2.029000 & 2.595000 & 3.920000 \\
\hline N9 & 2.260000 & 3.223000 & 5.097000 \\
\hline N8 & 3.352000 & 3.971000 & 4.970000 \\
\hline $\mathrm{C} 18$ & 0.840000 & 1.726000 & 3.672000 \\
\hline H17 & 0.399000 & 1.429000 & 4.625000 \\
\hline $\mathrm{H} 18$ & 1.155000 & 0.813000 & 3.164000 \\
\hline N11 & -0.158000 & 2.431000 & 2.837000 \\
\hline $\mathrm{H} 20$ & 0.112000 & 2.630000 & 1.885000 \\
\hline $\mathrm{H} 12$ & 3.207000 & 2.675000 & 2.000000 \\
\hline H1 & 6.309000 & 2.328000 & 4.025000 \\
\hline $\mathrm{C} 2$ & 8.026000 & 3.235000 & 4.964000 \\
\hline $\mathrm{C} 8$ & 8.656000 & 1.911000 & 5.403000 \\
\hline H6 & 9.584000 & 2.078000 & 5.948000 \\
\hline $\mathrm{H} 7$ & 7.975000 & 1.367000 & 6.057000 \\
\hline $\mathrm{N} 4$ & 8.959000 & 1.091000 & 4.239000 \\
\hline $\mathrm{C} 12$ & 9.841000 & 1.324000 & 3.241000 \\
\hline $\mathrm{C} 13$ & 9.716000 & 0.230000 & 2.413000 \\
\hline N6 & 8.788000 & -0.618000 & 2.936000 \\
\hline N5 & 8.326000 & -0.095000 & 4.063000 \\
\hline C17 & 10.428000 & -0.047000 & 1.128000 \\
\hline H15 & 10.955000 & 0.858000 & 0.814000 \\
\hline
\end{tabular}




\begin{tabular}{|c|c|c|c|}
\hline H16 & 9.691000 & -0.269000 & 0.356000 \\
\hline N10 & 11.395000 & -1.162000 & 1.218000 \\
\hline H19 & 11.034000 & -2.089000 & 1.095000 \\
\hline H11 & 10.469000 & 2.195000 & 3.199000 \\
\hline $\mathrm{C} 1$ & 12.689000 & -1.031000 & 1.592000 \\
\hline $\mathrm{O} 1$ & 13.163000 & 0.067000 & 1.781000 \\
\hline C3 & 13.503000 & -2.327000 & 1.728000 \\
\hline $\mathrm{C} 4$ & 12.731000 & -3.356000 & 2.613000 \\
\hline H5 & 11.886000 & -3.758000 & 2.053000 \\
\hline H6 & 13.383000 & -4.197000 & 2.853000 \\
\hline $\mathrm{N} 2$ & 12.222000 & -2.813000 & 3.863000 \\
\hline $\mathrm{C} 7$ & 12.893000 & -2.486000 & 4.986000 \\
\hline C8 & 11.930000 & -1.972000 & 5.822000 \\
\hline N4 & 10.728000 & -2.014000 & 5.191000 \\
\hline N3 & 10.908000 & -2.534000 & 3.985000 \\
\hline $\mathrm{C} 11$ & 12.117000 & -1.507000 & 7.227000 \\
\hline H14 & 13.005000 & -0.873000 & 7.276000 \\
\hline H15 & 11.261000 & -0.905000 & 7.534000 \\
\hline N8 & 12.287000 & -2.647000 & 8.157000 \\
\hline H18 & 11.489000 & -2.914000 & 8.710000 \\
\hline $\mathrm{H} 12$ & 13.946000 & -2.646000 & 5.135000 \\
\hline $\mathrm{C} 5$ & 14.881000 & -2.066000 & 2.411000 \\
\hline $\mathrm{H} 7$ & 15.401000 & -3.008000 & 2.590000 \\
\hline H8 & 15.531000 & -1.440000 & 1.805000 \\
\hline $\mathrm{H} 9$ & 14.753000 & -1.555000 & 3.365000 \\
\hline C6 & 13.732000 & -2.959000 & 0.318000 \\
\hline $\mathrm{H} 10$ & 14.407000 & -3.810000 & 0.421000 \\
\hline H11 & 12.783000 & -3.343000 & -0.056000 \\
\hline N5 & 14.279000 & -2.092000 & -0.724000 \\
\hline C9 & 14.897000 & -0.892000 & -0.629000 \\
\hline H13 & 15.044000 & -0.345000 & 0.286000 \\
\hline N6 & 14.228000 & -2.502000 & -2.016000 \\
\hline $\mathrm{N} 7$ & 14.816000 & -1.564000 & -2.745000 \\
\hline $\mathrm{C} 10$ & 15.241000 & -0.573000 & -1.919000 \\
\hline $\mathrm{C} 12$ & 16.021000 & 0.614000 & -2.374000 \\
\hline H16 & 15.724000 & 0.892000 & -3.386000 \\
\hline H17 & 15.794000 & 1.462000 & -1.723000 \\
\hline N9 & 17.476999 & 0.352000 & -2.330000 \\
\hline H19 & 17.933001 & 0.140000 & -3.204000 \\
\hline $\mathrm{C} 1$ & -1.202000 & 3.125000 & 3.337000 \\
\hline $\mathrm{O} 1$ & -1.590000 & 2.924000 & 4.474000 \\
\hline $\mathrm{C} 3$ & -1.759000 & 4.235000 & 2.437000 \\
\hline $\mathrm{C} 4$ & -0.614000 & 5.237000 & 2.053000 \\
\hline H5 & 0.027000 & 4.762000 & 1.309000 \\
\hline H6 & -1.059000 & 6.107000 & 1.568000 \\
\hline $\mathrm{N} 2$ & 0.286000 & 5.739000 & 3.098000 \\
\hline $\mathrm{C} 7$ & 1.405000 & 6.482000 & 2.922000 \\
\hline C8 & 1.878000 & 6.706000 & 4.200000 \\
\hline N4 & 1.043000 & 6.100000 & 5.079000 \\
\hline N3 & 0.076000 & 5.506000 & 4.410000 \\
\hline C11 & 3.081000 & 7.497000 & 4.616000 \\
\hline H14 & 3.811000 & 7.514000 & 3.807000 \\
\hline H15 & 2.772000 & 8.531000 & 4.778000 \\
\hline
\end{tabular}




\begin{tabular}{|c|c|c|c|}
\hline N8 & 3.746000 & 7.018000 & 5.849000 \\
\hline $\mathrm{H} 18$ & 3.270000 & 6.270000 & 6.346000 \\
\hline $\mathrm{H} 12$ & 1.757000 & 6.798000 & 1.958000 \\
\hline C5 & -2.904000 & 5.014000 & 3.148000 \\
\hline $\mathrm{H7}$ & -3.311000 & 5.777000 & 2.484000 \\
\hline H8 & -3.713000 & 4.339000 & 3.429000 \\
\hline H9 & -2.547000 & 5.505000 & 4.052000 \\
\hline C6 & -2.329000 & 3.640000 & 1.114000 \\
\hline $\mathrm{H} 10$ & -2.695000 & 4.458000 & 0.491000 \\
\hline H11 & -1.531000 & 3.151000 & 0.553000 \\
\hline N5 & -3.407000 & 2.674000 & 1.268000 \\
\hline C9 & -3.333000 & 1.370000 & 1.625000 \\
\hline $\mathrm{H} 13$ & -2.418000 & 0.869000 & 1.878000 \\
\hline N6 & -4.679000 & 3.032000 & 0.984000 \\
\hline N7 & -5.433000 & 1.957000 & 1.165000 \\
\hline $\mathrm{C} 10$ & -4.637000 & 0.925000 & 1.558000 \\
\hline C12 & -5.141000 & -0.458000 & 1.823000 \\
\hline H16 & -5.251000 & -0.983000 & 0.871000 \\
\hline H17 & -4.399000 & -1.014000 & 2.399000 \\
\hline N9 & -6.431000 & -0.519000 & 2.541000 \\
\hline H19 & -6.944000 & 0.346000 & 2.630000 \\
\hline $\mathrm{C} 1$ & 8.513000 & 9.767000 & 0.504000 \\
\hline $\mathrm{O} 1$ & 7.895000 & 8.754000 & 0.757000 \\
\hline C3 & 7.828000 & 11.083000 & 0.103000 \\
\hline $\mathrm{C} 4$ & 8.101000 & 12.174000 & 1.180000 \\
\hline H5 & 9.171000 & 12.376000 & 1.228000 \\
\hline H6 & 7.610000 & 13.101000 & 0.883000 \\
\hline $\mathrm{N} 2$ & 7.660000 & 11.855000 & 2.527000 \\
\hline $\mathrm{C} 7$ & 6.594000 & 12.343000 & 3.201000 \\
\hline $\mathrm{C} 8$ & 6.684000 & 11.754000 & 4.447000 \\
\hline N4 & 7.779000 & 10.954000 & 4.472000 \\
\hline N3 & 8.386000 & 11.022000 & 3.299000 \\
\hline C11 & 5.817000 & 11.927000 & 5.652000 \\
\hline H14 & 5.388000 & 10.959000 & 5.909000 \\
\hline $\mathrm{H} 15$ & 6.447000 & 12.224000 & 6.493000 \\
\hline N8 & 4.727000 & 12.922000 & 5.492000 \\
\hline H18 & 3.781000 & 12.578000 & 5.528000 \\
\hline $\mathrm{H} 12$ & 5.903000 & 13.049000 & 2.782000 \\
\hline C5 & 6.291000 & 10.882000 & -0.019000 \\
\hline H7 & 5.803000 & 11.822000 & -0.283000 \\
\hline H8 & 6.056000 & 10.143000 & -0.784000 \\
\hline H9 & 5.871000 & 10.531000 & 0.924000 \\
\hline C6 & 8.377000 & 11.616000 & -1.254000 \\
\hline $\mathrm{H} 10$ & 7.673000 & 12.327000 & -1.688000 \\
\hline H11 & 9.307000 & 12.158000 & -1.076000 \\
\hline N5 & 8.658000 & 10.596000 & -2.249000 \\
\hline C9 & 7.816000 & 9.743000 & -2.874000 \\
\hline H13 & 6.751000 & 9.744000 & -2.744000 \\
\hline N6 & 9.937000 & 10.382000 & -2.615000 \\
\hline N7 & 9.929000 & 9.385000 & -3.483000 \\
\hline C10 & 8.649000 & 8.971000 & -3.654000 \\
\hline $\mathrm{C} 12$ & 8.283000 & 7.847000 & -4.563000 \\
\hline H16 & 8.748000 & 6.934000 & -4.186000 \\
\hline
\end{tabular}




\begin{tabular}{|c|c|c|c|}
\hline H17 & 7.201000 & 7.701000 & -4.530000 \\
\hline N9 & 8.699000 & 8.066000 & -5.966000 \\
\hline H19 & 9.325000 & 7.386000 & -6.363000 \\
\hline $\mathrm{C} 1$ & 13.330000 & -3.510000 & 8.118000 \\
\hline $\mathrm{O} 1$ & 14.312000 & -3.256000 & 7.451000 \\
\hline $\mathrm{C} 3$ & 13.186000 & -4.829000 & 8.893000 \\
\hline $\mathrm{C} 4$ & 12.373000 & -5.848000 & 8.036000 \\
\hline H5 & 12.894000 & -6.007000 & 7.090000 \\
\hline H6 & 12.341000 & -6.809000 & 8.550000 \\
\hline $\mathrm{N} 2$ & 11.004000 & -5.470000 & 7.728000 \\
\hline C7 & 9.879000 & -5.687000 & 8.453000 \\
\hline C8 & 8.885000 & -5.072000 & 7.720000 \\
\hline N4 & 9.441000 & -4.524000 & 6.604000 \\
\hline N3 & 10.735000 & -4.778000 & 6.601000 \\
\hline C11 & 7.413000 & -4.975000 & 7.987000 \\
\hline H14 & 7.067000 & -5.911000 & 8.436000 \\
\hline H15 & 7.220000 & -4.181000 & 8.711000 \\
\hline N8 & 6.654000 & -4.726000 & 6.740000 \\
\hline H18 & 6.442000 & -3.769000 & 6.535000 \\
\hline H12 & 9.869000 & -6.248000 & 9.370000 \\
\hline C5 & 12.502000 & -4.610000 & 10.274000 \\
\hline H7 & 12.473000 & -5.547000 & 10.832000 \\
\hline H8 & 13.054000 & -3.875000 & 10.861000 \\
\hline H9 & 11.481000 & -4.246000 & 10.165000 \\
\hline C6 & 14.585000 & -5.476000 & 9.126000 \\
\hline H10 & 14.448000 & -6.456000 & 9.587000 \\
\hline H11 & 15.073000 & -5.645000 & 8.165000 \\
\hline N5 & 15.506000 & -4.721000 & 9.961000 \\
\hline C9 & 16.364000 & -3.728000 & 9.620000 \\
\hline $\mathrm{H} 13$ & 16.412001 & -3.334000 & 8.619000 \\
\hline N6 & 15.611000 & -5.028000 & 11.271000 \\
\hline N7 & 16.525999 & -4.231000 & 11.781000 \\
\hline C10 & 17.013000 & -3.424000 & 10.799000 \\
\hline $\mathrm{C} 12$ & 18.104000 & -2.436000 & 11.088000 \\
\hline H16 & 18.886999 & -2.533000 & 10.332000 \\
\hline H17 & 17.698999 & -1.424000 & 10.998000 \\
\hline N9 & 18.688999 & -2.634000 & 12.438000 \\
\hline H19 & 19.232000 & -3.470000 & 12.550000 \\
\hline $\mathrm{C} 1$ & 18.177000 & 0.113000 & -1.197000 \\
\hline $\mathrm{O} 1$ & 17.667999 & 0.317000 & -0.115000 \\
\hline C3 & 19.618999 & -0.400000 & -1.348000 \\
\hline $\mathrm{C} 4$ & 19.785000 & -1.366000 & -2.564000 \\
\hline H5 & 19.823999 & -0.776000 & -3.482000 \\
\hline H6 & 20.742001 & -1.885000 & -2.491000 \\
\hline $\mathrm{N} 2$ & 18.746000 & -2.372000 & -2.730000 \\
\hline C7 & 18.416000 & -3.433000 & -1.952000 \\
\hline $\mathrm{C} 8$ & 17.329000 & -3.990000 & -2.592000 \\
\hline $\mathrm{N} 4$ & 17.069000 & -3.265000 & -3.712000 \\
\hline N3 & 17.924000 & -2.273000 & -3.794000 \\
\hline C11 & 16.465000 & -5.154000 & -2.218000 \\
\hline H14 & 17.046000 & -6.077000 & -2.305000 \\
\hline H15 & 16.163000 & -5.060000 & -1.174000 \\
\hline N8 & 15.262000 & -5.216000 & -3.081000 \\
\hline
\end{tabular}




\begin{tabular}{|c|c|c|c|}
\hline H18 & 14.696000 & -4.384000 & -3.039000 \\
\hline $\mathrm{H} 12$ & 18.962000 & -3.709000 & -1.068000 \\
\hline C5 & 20.059999 & -1.169000 & -0.067000 \\
\hline $\mathrm{H7}$ & 21.086000 & -1.524000 & -0.171000 \\
\hline $\mathrm{H} 8$ & 20.007999 & -0.534000 & 0.818000 \\
\hline $\mathrm{H} 9$ & 19.410000 & -2.023000 & 0.114000 \\
\hline C6 & 20.562000 & 0.814000 & -1.589000 \\
\hline $\mathrm{H} 10$ & 21.506001 & 0.441000 & -1.990000 \\
\hline H11 & 20.132000 & 1.467000 & -2.350000 \\
\hline N5 & 20.872999 & 1.630000 & -0.423000 \\
\hline C9 & 20.061001 & 2.307000 & 0.430000 \\
\hline $\mathrm{H} 13$ & 18.988001 & 2.283000 & 0.361000 \\
\hline N6 & 22.169001 & 1.782000 & -0.084000 \\
\hline N7 & 22.202000 & 2.548000 & 0.983000 \\
\hline C10 & 20.933001 & 2.890000 & 1.331000 \\
\hline $\mathrm{C} 12$ & 20.691000 & 3.788000 & 2.508000 \\
\hline H16 & 19.985001 & 4.572000 & 2.228000 \\
\hline H17 & 20.223000 & 3.206000 & 3.307000 \\
\hline N9 & 21.951000 & 4.406000 & 2.996000 \\
\hline H19 & 22.381001 & 5.051000 & 2.359000 \\
\hline $\mathrm{C} 1$ & 4.785000 & 7.649000 & 6.443000 \\
\hline O1 & 5.359000 & 8.511000 & 5.815000 \\
\hline $\mathrm{C} 3$ & 5.189000 & 7.264000 & 7.884000 \\
\hline $\mathrm{C} 4$ & 5.919000 & 8.468000 & 8.578000 \\
\hline H5 & 5.349000 & 9.379000 & 8.384000 \\
\hline H6 & 5.908000 & 8.319000 & 9.657000 \\
\hline $\mathrm{N} 2$ & 7.312000 & 8.732000 & 8.235000 \\
\hline $\mathrm{C7}$ & 8.427000 & 8.184000 & 8.779000 \\
\hline $\mathrm{C} 8$ & 9.467000 & 8.847000 & 8.159000 \\
\hline N4 & 8.949000 & 9.752000 & 7.289000 \\
\hline N3 & 7.631000 & 9.693000 & 7.343000 \\
\hline C11 & 10.940000 & 8.649000 & 8.345000 \\
\hline H14 & 11.197000 & 8.888000 & 9.380000 \\
\hline H15 & 11.179000 & 7.595000 & 8.196000 \\
\hline N8 & 11.767000 & 9.465000 & 7.426000 \\
\hline H18 & 11.876000 & 9.101000 & 6.488000 \\
\hline H12 & 8.399000 & 7.411000 & 9.524000 \\
\hline C5 & 6.102000 & 6.009000 & 7.861000 \\
\hline $\mathrm{H} 7$ & 6.303000 & 5.666000 & 8.876000 \\
\hline $\mathrm{H} 8$ & 5.634000 & 5.202000 & 7.296000 \\
\hline H9 & 7.052000 & 6.227000 & 7.388000 \\
\hline C6 & 3.954000 & 6.981000 & 8.801000 \\
\hline $\mathrm{H} 10$ & 4.278000 & 6.932000 & 9.841000 \\
\hline H11 & 3.259000 & 7.820000 & 8.723000 \\
\hline N5 & 3.223000 & 5.752000 & 8.551000 \\
\hline C9 & 3.532000 & 4.469000 & 8.866000 \\
\hline $\mathrm{H} 13$ & 4.411000 & 4.194000 & 9.418000 \\
\hline N6 & 2.056000 & 5.827000 & 7.896000 \\
\hline N7 & 1.623000 & 4.603000 & 7.752000 \\
\hline $\mathrm{C} 10$ & 2.491000 & 3.740000 & 8.332000 \\
\hline $\mathrm{C} 12$ & 2.180000 & 2.276000 & 8.341000 \\
\hline H16 & 2.620000 & 1.816000 & 7.455000 \\
\hline H17 & 2.625000 & 1.812000 & 9.226000 \\
\hline
\end{tabular}




\begin{tabular}{|c|c|c|c|}
\hline N9 & 0.714000 & 2.061000 & 8.349000 \\
\hline H19 & 0.285000 & 2.003000 & 7.445000 \\
\hline $\mathrm{C} 1$ & -7.068000 & -1.673000 & 2.845000 \\
\hline $\mathrm{O} 1$ & -6.498000 & -2.724000 & 2.648000 \\
\hline $\mathrm{C} 3$ & -8.488000 & -1.592000 & 3.442000 \\
\hline $\mathrm{C} 4$ & -9.464000 & -0.778000 & 2.530000 \\
\hline H5 & -9.452000 & -1.217000 & 1.530000 \\
\hline H6 & -10.481000 & -0.887000 & 2.908000 \\
\hline $\mathrm{N} 2$ & -9.226000 & 0.651000 & 2.395000 \\
\hline $\mathrm{C7}$ & -9.610000 & 1.677000 & 3.197000 \\
\hline $\mathrm{C} 8$ & -9.095000 & 2.799000 & 2.575000 \\
\hline N4 & -8.445000 & 2.402000 & 1.454000 \\
\hline N3 & -8.531000 & 1.099000 & 1.333000 \\
\hline C11 & -9.166000 & 4.255000 & 2.925000 \\
\hline H14 & -10.137000 & 4.474000 & 3.376000 \\
\hline H15 & -8.402000 & 4.480000 & 3.674000 \\
\hline N8 & -8.967000 & 5.117000 & 1.731000 \\
\hline H18 & -9.751000 & 5.148000 & 1.101000 \\
\hline $\mathrm{H} 12$ & -10.207000 & 1.536000 & 4.080000 \\
\hline $\mathrm{C} 5$ & -8.420000 & -0.965000 & 4.860000 \\
\hline $\mathrm{H7}$ & -9.415000 & -0.928000 & 5.305000 \\
\hline H8 & -7.771000 & -1.556000 & 5.507000 \\
\hline H9 & -8.015000 & 0.046000 & 4.821000 \\
\hline C6 & -9.134000 & -3.015000 & 3.532000 \\
\hline H10 & -10.190000 & -2.903000 & 3.782000 \\
\hline H11 & -9.094000 & -3.486000 & 2.548000 \\
\hline N5 & -8.575000 & -3.959000 & 4.492000 \\
\hline C9 & -7.555000 & -4.838000 & 4.339000 \\
\hline $\mathrm{H} 13$ & -6.950000 & -4.855000 & 3.449000 \\
\hline N6 & -9.168000 & -4.112000 & 5.697000 \\
\hline N7 & -8.520000 & -5.071000 & 6.331000 \\
\hline $\mathrm{C} 10$ & -7.528000 & -5.538000 & 5.527000 \\
\hline $\mathrm{C} 12$ & -6.659000 & -6.675000 & 5.962000 \\
\hline H16 & -6.291000 & -7.211000 & 5.084000 \\
\hline H17 & -5.785000 & -6.273000 & 6.481000 \\
\hline N9 & -7.398000 & -7.611000 & 6.843000 \\
\hline H19 & -8.178000 & -8.078000 & 6.415000 \\
\hline $\mathrm{C} 1$ & 4.913000 & 14.251000 & 5.324000 \\
\hline $\mathrm{O} 1$ & 6.037000 & 14.707000 & 5.282000 \\
\hline C3 & 3.673000 & 15.148000 & 5.189000 \\
\hline $\mathrm{C} 4$ & 3.467000 & 15.952000 & 6.507000 \\
\hline H5 & 4.398000 & 16.473000 & 6.744000 \\
\hline H6 & 2.713000 & 16.722000 & 6.340000 \\
\hline $\mathrm{N} 2$ & 3.052000 & 15.219000 & 7.694000 \\
\hline C7 & 1.799000 & 14.880000 & 8.087000 \\
\hline $\mathrm{C} 8$ & 1.974000 & 14.332000 & 9.341000 \\
\hline N4 & 3.296000 & 14.373000 & 9.656000 \\
\hline N3 & 3.957000 & 14.924000 & 8.651000 \\
\hline C11 & 0.971000 & 13.826000 & 10.327000 \\
\hline H14 & -0.031000 & 14.144000 & 10.024000 \\
\hline H15 & 0.979000 & 12.734000 & 10.325000 \\
\hline N8 & 1.271000 & 14.330000 & 11.687000 \\
\hline $\mathrm{H} 18$ & 1.960000 & 13.811000 & 12.202000 \\
\hline
\end{tabular}




\begin{tabular}{|c|c|c|c|}
\hline H12 & 0.926000 & 15.046000 & 7.483000 \\
\hline $\mathrm{C} 5$ & 2.381000 & 14.355000 & 4.831000 \\
\hline $\mathrm{H} 7$ & 1.543000 & 15.040000 & 4.700000 \\
\hline H8 & 2.518000 & 13.793000 & 3.906000 \\
\hline H9 & 2.122000 & 13.646000 & 5.617000 \\
\hline C6 & 3.928000 & 16.226000 & 4.090000 \\
\hline H10 & 3.112000 & 16.950001 & 4.113000 \\
\hline H11 & 4.836000 & 16.777000 & 4.344000 \\
\hline N5 & 4.047000 & 15.781000 & 2.708000 \\
\hline C9 & 3.063000 & 15.596000 & 1.796000 \\
\hline H13 & 2.017000 & 15.634000 & 2.037000 \\
\hline N6 & 5.265000 & 15.700000 & 2.128000 \\
\hline N7 & 5.071000 & 15.446000 & 0.843000 \\
\hline $\mathrm{C} 10$ & 3.735000 & 15.377000 & 0.611000 \\
\hline C12 & 3.209000 & 15.243000 & -0.779000 \\
\hline H16 & 3.566000 & 14.310000 & -1.220000 \\
\hline H17 & 2.116000 & 15.203000 & -0.757000 \\
\hline N9 & 3.649000 & 16.399000 & -1.593000 \\
\hline H19 & 4.387000 & 16.228001 & -2.256000 \\
\hline $\mathrm{C} 1$ & 8.341000 & 9.129000 & -6.722000 \\
\hline $\mathrm{O} 1$ & 7.565000 & 9.956000 & -6.291000 \\
\hline C3 & 8.917000 & 9.178000 & -8.141000 \\
\hline $\mathrm{C} 4$ & 10.438000 & 8.834000 & -8.151000 \\
\hline H5 & 10.615000 & 7.858000 & -7.699000 \\
\hline H6 & 10.760000 & 8.751000 & -9.190000 \\
\hline $\mathrm{N} 2$ & 11.314000 & 9.797000 & -7.502000 \\
\hline $\mathrm{C} 7$ & 11.650000 & 9.944000 & -6.196000 \\
\hline $\mathrm{C} 8$ & 12.578000 & 10.968000 & -6.204000 \\
\hline $\mathrm{N} 4$ & 12.753000 & 11.384000 & -7.485000 \\
\hline N3 & 11.992000 & 10.666000 & -8.280000 \\
\hline $\mathrm{C} 11$ & 13.403000 & 11.581000 & -5.115000 \\
\hline H14 & 12.858000 & 12.427000 & -4.689000 \\
\hline H15 & 13.551000 & 10.854000 & -4.313000 \\
\hline N8 & 14.721000 & 12.026000 & -5.642000 \\
\hline H18 & 15.216000 & 11.331000 & -6.171000 \\
\hline H12 & 11.213000 & 9.365000 & -5.402000 \\
\hline $\mathrm{C} 5$ & 8.721000 & 10.591000 & -8.767000 \\
\hline $\mathrm{H} 7$ & 9.144000 & 10.621000 & -9.772000 \\
\hline $\mathrm{H} 8$ & 7.664000 & 10.851000 & -8.830000 \\
\hline H9 & 9.209000 & 11.354000 & -8.160000 \\
\hline C6 & 8.199000 & 8.117000 & -9.023000 \\
\hline H10 & 8.701000 & 8.069000 & -9.991000 \\
\hline H11 & 8.292000 & 7.131000 & -8.567000 \\
\hline N5 & 6.787000 & 8.363000 & -9.271000 \\
\hline C9 & 5.715000 & 8.159000 & -8.467000 \\
\hline H13 & 5.799000 & 7.791000 & -7.461000 \\
\hline N6 & 6.411000 & 8.847000 & -10.474000 \\
\hline N7 & 5.100000 & 8.965000 & -10.450000 \\
\hline C10 & 4.639000 & 8.553000 & -9.239000 \\
\hline C12 & 3.170000 & 8.546000 & -8.947000 \\
\hline H16 & 2.911000 & 7.633000 & -8.406000 \\
\hline H17 & 2.935000 & 9.386000 & -8.288000 \\
\hline N9 & 2.365000 & 8.626000 & -10.192000 \\
\hline
\end{tabular}




\begin{tabular}{|c|c|c|c|}
\hline H19 & 2.551000 & 7.902000 & -10.862000 \\
\hline $\mathrm{C} 1$ & 6.606000 & -5.656000 & 5.767000 \\
\hline $\mathrm{C} 2$ & 6.521000 & -5.218000 & 4.302000 \\
\hline C3 & 5.140000 & -5.708000 & 3.752000 \\
\hline N1 & 4.838000 & -7.196000 & 3.967000 \\
\hline $\mathrm{H} 3$ & 5.079000 & -7.476000 & 4.946000 \\
\hline $\mathrm{H} 4$ & 5.407000 & -7.813000 & 3.360000 \\
\hline H16 & 3.841000 & -7.438000 & 3.809000 \\
\hline $\mathrm{H} 1$ & 5.061000 & -5.514000 & 2.676000 \\
\hline $\mathrm{H} 2$ & 4.335000 & -5.162000 & 4.257000 \\
\hline $\mathrm{C} 4$ & 6.692000 & -3.671000 & 4.104000 \\
\hline $\mathrm{N} 2$ & 8.063000 & -3.085000 & 4.445000 \\
\hline $\mathrm{H} 7$ & 8.829000 & -3.431000 & 3.840000 \\
\hline $\mathrm{H} 8$ & 8.409000 & -3.297000 & 5.405000 \\
\hline H17 & 8.120000 & -2.045000 & 4.328000 \\
\hline H5 & 6.513000 & -3.414000 & 3.054000 \\
\hline H6 & 5.954000 & -3.128000 & 4.702000 \\
\hline C5 & 7.660000 & -5.924000 & 3.509000 \\
\hline $\mathrm{H} 9$ & 8.637000 & -5.681000 & 3.932000 \\
\hline $\mathrm{H} 10$ & 7.562000 & -7.010000 & 3.554000 \\
\hline H11 & 7.647000 & -5.623000 & 2.460000 \\
\hline $\mathrm{O} 1$ & 6.623000 & -6.835000 & 6.039000 \\
\hline $\mathrm{C} 1$ & 18.254999 & -1.959000 & 13.519000 \\
\hline $\mathrm{C} 2$ & 18.386999 & -2.596000 & 14.908000 \\
\hline $\mathrm{C} 3$ & 18.743999 & -4.125000 & 14.870000 \\
\hline N1 & 17.671000 & -5.077000 & 14.326000 \\
\hline $\mathrm{H} 3$ & 17.349001 & -4.823000 & 13.364000 \\
\hline $\mathrm{H} 4$ & 16.815001 & -5.085000 & 14.910000 \\
\hline H16 & 18.000000 & -6.060000 & 14.274000 \\
\hline $\mathrm{H} 1$ & 18.962000 & -4.469000 & 15.888000 \\
\hline $\mathrm{H} 2$ & 19.650999 & -4.287000 & 14.277000 \\
\hline $\mathrm{C} 4$ & 19.487000 & -1.786000 & 15.669000 \\
\hline $\mathrm{N} 2$ & 19.233999 & -0.277000 & 15.753000 \\
\hline $\mathrm{H} 7$ & 18.466000 & -0.041000 & 16.408001 \\
\hline $\mathrm{H} 8$ & 18.934000 & 0.095000 & 14.823000 \\
\hline H17 & 20.070000 & 0.259000 & 16.052000 \\
\hline H5 & 19.604000 & -2.153000 & 16.695000 \\
\hline H6 & 20.448000 & -1.906000 & 15.155000 \\
\hline $\mathrm{C} 5$ & 17.034000 & -2.442000 & 15.667000 \\
\hline H9 & 16.757999 & -1.393000 & 15.784000 \\
\hline $\mathrm{H} 10$ & 16.222000 & -2.917000 & 15.114000 \\
\hline H11 & 17.091999 & -2.890000 & 16.660999 \\
\hline $\mathrm{O} 1$ & 17.809999 & -0.839000 & 13.416000 \\
\hline $\mathrm{C} 1$ & 15.216000 & -6.000000 & -4.173000 \\
\hline $\mathrm{C} 2$ & 14.413000 & -5.549000 & -5.402000 \\
\hline C3 & 13.317000 & -6.636000 & -5.665000 \\
\hline N1 & 13.840000 & -8.066000 & -5.838000 \\
\hline $\mathrm{H} 3$ & 14.532000 & -8.289000 & -5.084000 \\
\hline $\mathrm{H} 4$ & 14.352000 & -8.196000 & -6.729000 \\
\hline H16 & 13.093000 & -8.784000 & -5.802000 \\
\hline $\mathrm{H} 1$ & 12.738000 & -6.395000 & -6.564000 \\
\hline $\mathrm{H} 2$ & 12.626000 & -6.666000 & -4.815000 \\
\hline $\mathrm{C} 4$ & 13.743000 & -4.140000 & -5.239000 \\
\hline
\end{tabular}




\begin{tabular}{|c|c|c|c|}
\hline $\mathrm{N} 2$ & 14.661000 & -2.918000 & -5.311000 \\
\hline $\mathrm{H} 7$ & 15.132000 & -2.810000 & -6.227000 \\
\hline $\mathrm{H} 8$ & 15.423000 & -2.942000 & -4.594000 \\
\hline $\mathrm{H} 17$ & 14.155000 & -2.034000 & -5.114000 \\
\hline $\mathrm{H} 5$ & 13.000000 & -3.999000 & -6.033000 \\
\hline H6 & 13.211000 & -4.082000 & -4.282000 \\
\hline C5 & 15.374000 & -5.501000 & -6.627000 \\
\hline H9 & 16.204000 & -4.816000 & -6.444000 \\
\hline H10 & 15.818000 & -6.478000 & -6.823000 \\
\hline H11 & 14.844000 & -5.181000 & -7.527000 \\
\hline $\mathrm{O} 1$ & 15.789000 & -7.066000 & -4.195000 \\
\hline $\mathrm{C} 1$ & 22.667999 & 3.884000 & 4.010000 \\
\hline $\mathrm{C} 2$ & 24.186001 & 4.119000 & 4.055000 \\
\hline $\mathrm{C} 3$ & 24.791000 & 4.645000 & 2.704000 \\
\hline N1 & 24.822001 & 3.668000 & 1.522000 \\
\hline $\mathrm{H} 3$ & 23.877001 & 3.298000 & 1.275000 \\
\hline $\mathrm{H} 4$ & 25.408001 & 2.835000 & 1.711000 \\
\hline H16 & 25.198000 & 4.102000 & 0.658000 \\
\hline H1 & 25.833000 & 4.943000 & 2.871000 \\
\hline $\mathrm{H} 2$ & 24.253000 & 5.539000 & 2.371000 \\
\hline $\mathrm{C} 4$ & 24.445000 & 5.143000 & 5.206000 \\
\hline N2 & 23.885000 & 4.729000 & 6.568000 \\
\hline $\mathrm{H} 7$ & 24.427999 & 3.959000 & 6.997000 \\
\hline H8 & 22.909000 & 4.367000 & 6.467000 \\
\hline H17 & 23.867001 & 5.511000 & 7.250000 \\
\hline H5 & 25.518999 & 5.323000 & 5.337000 \\
\hline H6 & 23.971001 & 6.099000 & 4.957000 \\
\hline C5 & 24.899000 & 2.778000 & 4.405000 \\
\hline H9 & 24.570999 & 2.390000 & 5.371000 \\
\hline $\mathrm{H} 10$ & 24.664000 & 2.008000 & 3.667000 \\
\hline H11 & 25.982000 & 2.910000 & 4.443000 \\
\hline O1 & 22.129000 & 3.288000 & 4.914000 \\
\hline $\mathrm{C} 1$ & 12.206000 & 10.707000 & 7.712000 \\
\hline $\mathrm{C} 2$ & 12.794000 & 11.551000 & 6.576000 \\
\hline C3 & 11.635000 & 12.263000 & 5.810000 \\
\hline N1 & 10.448000 & 11.404000 & 5.416000 \\
\hline $\mathrm{H} 3$ & 9.936000 & 10.997000 & 6.228000 \\
\hline $\mathrm{H} 4$ & 10.694000 & 10.574000 & 4.833000 \\
\hline H16 & 9.713000 & 11.889000 & 4.863000 \\
\hline H1 & 12.022000 & 12.718000 & 4.893000 \\
\hline $\mathrm{H} 2$ & 11.221000 & 13.062000 & 6.436000 \\
\hline $\mathrm{C} 4$ & 13.711000 & 12.677000 & 7.168000 \\
\hline $\mathrm{N} 2$ & 14.574000 & 12.303000 & 8.371000 \\
\hline $\mathrm{H} 7$ & 15.298000 & 11.598000 & 8.151000 \\
\hline H8 & 13.961000 & 11.901000 & 9.116000 \\
\hline H17 & 15.050000 & 13.126000 & 8.785000 \\
\hline H5 & 14.371000 & 13.086000 & 6.394000 \\
\hline H6 & 13.072000 & 13.491000 & 7.528000 \\
\hline C5 & 13.635000 & 10.694000 & 5.590000 \\
\hline H9 & 14.434000 & 10.171000 & 6.120000 \\
\hline $\mathrm{H} 10$ & 13.022000 & 9.949000 & 5.083000 \\
\hline H11 & 14.088000 & 11.326000 & 4.825000 \\
\hline O1 & 12.123000 & 11.158000 & 8.833000 \\
\hline
\end{tabular}




\begin{tabular}{|c|c|c|c|}
\hline $\mathrm{C} 1$ & -0.024000 & 2.462000 & 9.403000 \\
\hline $\mathrm{C} 2$ & -1.427000 & 3.038000 & 9.168000 \\
\hline $\mathrm{C} 3$ & -1.793000 & 3.203000 & 7.653000 \\
\hline N1 & -1.052000 & 4.288000 & 6.867000 \\
\hline $\mathrm{H} 3$ & -0.016000 & 4.261000 & 6.982000 \\
\hline $\mathrm{H} 4$ & -1.319000 & 5.254000 & 7.124000 \\
\hline H16 & -1.196000 & 4.193000 & 5.840000 \\
\hline $\mathrm{H} 1$ & -2.858000 & 3.441000 & 7.557000 \\
\hline $\mathrm{H} 2$ & -1.628000 & 2.261000 & 7.119000 \\
\hline $\mathrm{C} 4$ & -2.443000 & 2.078000 & 9.867000 \\
\hline $\mathrm{N} 2$ & -2.167000 & 1.801000 & 11.347000 \\
\hline $\mathrm{H7}$ & -2.353000 & 2.626000 & 11.943000 \\
\hline $\mathrm{H} 8$ & -1.157000 & 1.573000 & 11.490000 \\
\hline H17 & -2.728000 & 1.016000 & 11.725000 \\
\hline H5 & -3.463000 & 2.472000 & 9.796000 \\
\hline H6 & -2.422000 & 1.103000 & 9.367000 \\
\hline C5 & -1.506000 & 4.439000 & 9.842000 \\
\hline H9 & -1.319000 & 4.377000 & 10.915000 \\
\hline $\mathrm{H} 10$ & -0.748000 & 5.110000 & 9.432000 \\
\hline H11 & -2.489000 & 4.889000 & 9.692000 \\
\hline $\mathrm{O} 1$ & 0.415000 & 2.385000 & 10.529000 \\
\hline $\mathrm{C} 1$ & -7.742000 & 5.464000 & 1.282000 \\
\hline $\mathrm{C} 2$ & -7.635000 & 5.875000 & -0.189000 \\
\hline C3 & -7.944000 & 4.635000 & -1.094000 \\
\hline N1 & -7.086000 & 3.389000 & -0.868000 \\
\hline H3 & -7.236000 & 2.975000 & 0.082000 \\
\hline $\mathrm{H} 4$ & -6.066000 & 3.564000 & -0.910000 \\
\hline H16 & -7.303000 & 2.624000 & -1.533000 \\
\hline $\mathrm{H} 1$ & -7.828000 & 4.906000 & -2.150000 \\
\hline $\mathrm{H} 2$ & -8.980000 & 4.308000 & -0.946000 \\
\hline $\mathrm{C} 4$ & -8.630000 & 7.042000 & -0.499000 \\
\hline $\mathrm{N} 2$ & -8.493000 & 8.301000 & 0.361000 \\
\hline $\mathrm{H} 7$ & -7.566000 & 8.752000 & 0.253000 \\
\hline H8 & -8.618000 & 8.107000 & 1.374000 \\
\hline H17 & -9.198000 & 9.023000 & 0.109000 \\
\hline H5 & -8.510000 & 7.360000 & -1.542000 \\
\hline H6 & -9.664000 & 6.697000 & -0.382000 \\
\hline C5 & -6.192000 & 6.362000 & -0.519000 \\
\hline $\mathrm{H} 9$ & -5.916000 & 7.215000 & 0.102000 \\
\hline $\mathrm{H} 10$ & -5.460000 & 5.575000 & -0.325000 \\
\hline H11 & -6.112000 & 6.655000 & -1.567000 \\
\hline O1 & -6.775000 & 5.427000 & 2.004000 \\
\hline $\mathrm{C} 1$ & -7.230000 & -7.656000 & 8.179000 \\
\hline $\mathrm{C} 2$ & -8.355000 & -8.233000 & 9.042000 \\
\hline C3 & -9.759000 & -8.139000 & 8.349000 \\
\hline N1 & -10.269000 & -6.727000 & 8.027000 \\
\hline $\mathrm{H} 3$ & -9.621000 & -6.189000 & 7.408000 \\
\hline $\mathrm{H} 4$ & -10.396000 & -6.148000 & 8.877000 \\
\hline H16 & -11.186000 & -6.739000 & 7.541000 \\
\hline $\mathrm{H} 1$ & -10.516000 & -8.599000 & 8.996000 \\
\hline $\mathrm{H} 2$ & -9.757000 & -8.699000 & 7.407000 \\
\hline $\mathrm{C} 4$ & -7.957000 & -9.711000 & 9.357000 \\
\hline $\mathrm{N} 2$ & -6.572000 & -9.875000 & 9.993000 \\
\hline
\end{tabular}




\begin{tabular}{|c|c|c|c|}
\hline $\mathrm{H7}$ & -6.554000 & -9.565000 & 10.982000 \\
\hline H8 & -5.863000 & -9.287000 & 9.501000 \\
\hline H17 & -6.233000 & -10.855000 & 9.975000 \\
\hline H5 & -8.685000 & -10.177000 & 10.032000 \\
\hline H6 & -7.938000 & -10.290000 & 8.426000 \\
\hline $\mathrm{C} 5$ & -8.446000 & -7.445000 & 10.384000 \\
\hline H9 & -7.515000 & -7.509000 & 10.951000 \\
\hline $\mathrm{H} 10$ & -8.628000 & -6.384000 & 10.201000 \\
\hline H11 & -9.250000 & -7.835000 & 11.010000 \\
\hline $\mathrm{O} 1$ & -6.191000 & -7.317000 & 8.693000 \\
\hline $\mathrm{C} 1$ & 0.866000 & 15.537000 & 12.127000 \\
\hline $\mathrm{C} 2$ & 1.649000 & 16.208000 & 13.258000 \\
\hline $\mathrm{C} 3$ & 0.814000 & 16.004999 & 14.563000 \\
\hline N1 & -0.638000 & 16.490000 & 14.478000 \\
\hline $\mathrm{H} 3$ & -1.095000 & 16.156000 & 13.600000 \\
\hline $\mathrm{H} 4$ & -0.708000 & 17.524000 & 14.452000 \\
\hline H16 & -1.220000 & 16.167000 & 15.276000 \\
\hline $\mathrm{H} 1$ & 1.281000 & 16.521999 & 15.410000 \\
\hline $\mathrm{H} 2$ & 0.767000 & 14.937000 & 14.806000 \\
\hline $\mathrm{C} 4$ & 3.108000 & 15.647000 & 13.403000 \\
\hline N2 & 4.029000 & 15.801000 & 12.183000 \\
\hline H7 & 4.178000 & 16.792999 & 11.921000 \\
\hline H8 & 3.665000 & 15.324000 & 11.328000 \\
\hline H17 & 4.972000 & 15.403000 & 12.353000 \\
\hline H5 & 3.612000 & 16.157000 & 14.233000 \\
\hline H6 & 3.084000 & 14.579000 & 13.650000 \\
\hline $\mathrm{C} 5$ & 1.769000 & 17.736000 & 12.974000 \\
\hline H9 & 2.273000 & 17.919001 & 12.023000 \\
\hline $\mathrm{H} 10$ & 0.788000 & 18.209000 & 12.904000 \\
\hline H11 & 2.329000 & 18.237000 & 13.766000 \\
\hline $\mathrm{O} 1$ & -0.122000 & 16.066999 & 11.678000 \\
\hline $\mathrm{C} 1$ & 3.246000 & 17.660000 & -1.329000 \\
\hline $\mathrm{C} 2$ & 4.124000 & 18.820999 & -1.800000 \\
\hline $\mathrm{C} 3$ & 5.640000 & 18.423000 & -1.813000 \\
\hline N1 & 6.219000 & 17.924000 & -0.481000 \\
\hline H3 & 5.727000 & 17.083000 & -0.106000 \\
\hline $\mathrm{H} 4$ & 6.174000 & 18.641001 & 0.266000 \\
\hline H16 & 7.219000 & 17.662001 & -0.566000 \\
\hline H1 & 6.243000 & 19.289000 & -2.112000 \\
\hline $\mathrm{H} 2$ & 5.818000 & 17.635000 & -2.553000 \\
\hline $\mathrm{C} 4$ & 3.609000 & 19.253000 & -3.210000 \\
\hline $\mathrm{N} 2$ & 2.128000 & 19.646999 & -3.265000 \\
\hline H7 & 1.934000 & 20.521000 & -2.740000 \\
\hline H8 & 1.515000 & 18.919001 & -2.844000 \\
\hline $\mathrm{H} 17$ & 1.795000 & 19.806999 & -4.235000 \\
\hline H5 & 4.182000 & 20.108999 & -3.586000 \\
\hline H6 & 3.738000 & 18.427000 & -3.921000 \\
\hline C5 & 3.979000 & 20.032000 & -0.827000 \\
\hline H9 & 2.948000 & 20.389999 & -0.781000 \\
\hline $\mathrm{H} 10$ & 4.258000 & 19.753000 & 0.190000 \\
\hline H11 & 4.610000 & 20.864000 & -1.145000 \\
\hline O1 & 2.211000 & 17.877001 & -0.749000 \\
\hline $\mathrm{C} 1$ & 15.096000 & 13.317000 & -5.722000 \\
\hline
\end{tabular}




\begin{tabular}{|c|c|c|c|}
\hline $\mathrm{C} 2$ & 16.135000 & 13.729000 & -6.773000 \\
\hline C3 & 17.489000 & 13.924000 & -6.019000 \\
\hline N1 & 17.430000 & 14.898000 & -4.839000 \\
\hline H3 & 16.605000 & 14.697000 & -4.233000 \\
\hline $\mathrm{H} 4$ & 17.316000 & 15.881000 & -5.148000 \\
\hline H16 & 18.278999 & 14.862000 & -4.243000 \\
\hline H1 & 18.266001 & 14.286000 & -6.701000 \\
\hline $\mathrm{H} 2$ & 17.822001 & 12.963000 & -5.607000 \\
\hline $\mathrm{C} 4$ & 16.292000 & 12.694000 & -7.943000 \\
\hline $\mathrm{N} 2$ & 15.078000 & 12.489000 & -8.858000 \\
\hline $\mathrm{H} 7$ & 14.781000 & 13.362000 & -9.330000 \\
\hline H8 & 14.238000 & 12.136000 & -8.348000 \\
\hline H17 & 15.264000 & 11.797000 & -9.609000 \\
\hline H5 & 17.108999 & 13.019000 & -8.599000 \\
\hline H6 & 16.568001 & 11.711000 & -7.549000 \\
\hline $\mathrm{C} 5$ & 15.719000 & 15.091000 & -7.408000 \\
\hline H9 & 14.733000 & 15.020000 & -7.871000 \\
\hline $\mathrm{H} 10$ & 15.655000 & 15.881000 & -6.658000 \\
\hline $\mathrm{H} 11$ & 16.440001 & 15.403000 & -8.166000 \\
\hline O1 & 14.680000 & 14.138000 & -4.938000 \\
\hline $\mathrm{C} 1$ & 1.668000 & 9.719000 & -10.557000 \\
\hline $\mathrm{C} 2$ & 1.326000 & 9.913000 & -12.037000 \\
\hline $\mathrm{C} 3$ & 2.278000 & 9.123000 & -13.004000 \\
\hline N1 & 3.759000 & 9.523000 & -12.996000 \\
\hline H3 & 4.210000 & 9.397000 & -12.062000 \\
\hline $\mathrm{H} 4$ & 3.905000 & 10.514000 & -13.257000 \\
\hline H16 & 4.324000 & 8.957000 & -13.658000 \\
\hline $\mathrm{H} 1$ & 1.934000 & 9.259000 & -14.036000 \\
\hline $\mathrm{H} 2$ & 2.235000 & 8.050000 & -12.789000 \\
\hline $\mathrm{C} 4$ & -0.159000 & 9.461000 & -12.212000 \\
\hline $\mathrm{N} 2$ & -1.153000 & 10.155000 & -11.276000 \\
\hline $\mathrm{H} 7$ & -1.302000 & 11.148000 & -11.530000 \\
\hline $\mathrm{H} 8$ & -0.795000 & 10.167000 & -10.295000 \\
\hline H17 & -2.085000 & 9.700000 & -11.265000 \\
\hline H5 & -0.502000 & 9.634000 & -13.238000 \\
\hline H6 & -0.242000 & 8.387000 & -12.004000 \\
\hline $\mathrm{C} 5$ & 1.436000 & 11.424000 & -12.405000 \\
\hline H9 & 0.753000 & 12.033000 & -11.809000 \\
\hline $\mathrm{H} 10$ & 2.442000 & 11.800000 & -12.209000 \\
\hline H11 & 1.201000 & 11.586000 & -13.459000 \\
\hline $\mathrm{O} 1$ & 1.246000 & 10.497000 & -9.735000 \\
\hline
\end{tabular}


Atomic coordinates for the optimized structure of $\mathbf{G 3} \mathbf{N a p h}_{\mathbf{N H}} \mathbf{2}$ with AMBER at MM level (parm99, gaff)

\begin{tabular}{|c|c|c|c|}
\hline \multirow[t]{2}{*}{ Energy $(\mathrm{kcal} / \mathrm{mol})$} & \multicolumn{3}{|l|}{721.08} \\
\hline & \multicolumn{3}{|c|}{ Coordinates (Angstroms) } \\
\hline Atomic type & $\mathrm{X}$ & $\mathrm{Y}$ & $\mathrm{X}$ \\
\hline $\mathrm{C} 1$ & 3.510000 & 0.452000 & 0.808000 \\
\hline C6 & 4.882000 & 0.669000 & 0.732000 \\
\hline $\mathrm{C} 5$ & 5.527000 & 0.686000 & -0.508000 \\
\hline $\mathrm{C} 4$ & 4.783000 & 0.492000 & -1.673000 \\
\hline $\mathrm{C} 3$ & 3.392000 & 0.295000 & -1.608000 \\
\hline $\mathrm{C} 7$ & 2.659000 & 0.143000 & -2.796000 \\
\hline $\mathrm{C} 10$ & 3.319000 & 0.196000 & -4.022000 \\
\hline C9 & 4.701000 & 0.355000 & -4.076000 \\
\hline C8 & 5.445000 & 0.496000 & -2.902000 \\
\hline $\mathrm{C} 11$ & 6.944000 & 0.621000 & -2.969000 \\
\hline $\mathrm{O} 1$ & 7.533000 & 0.585000 & -4.035000 \\
\hline N1 & 7.614000 & 0.793000 & -1.793000 \\
\hline $\mathrm{C} 12$ & 7.011000 & 0.899000 & -0.575000 \\
\hline $\mathrm{O} 2$ & 7.638000 & 1.173000 & 0.429000 \\
\hline C13 & 9.102000 & 0.814000 & -1.840000 \\
\hline H6 & 9.514000 & 0.500000 & -0.879000 \\
\hline $\mathrm{H} 7$ & 9.442000 & 0.077000 & -2.569000 \\
\hline C14 & 9.675000 & 2.198000 & -2.208000 \\
\hline H8 & 9.378000 & 2.937000 & -1.462000 \\
\hline H9 & 9.260000 & 2.501000 & -3.171000 \\
\hline $\mathrm{C} 27$ & 11.215000 & 2.159000 & -2.311000 \\
\hline $\mathrm{N} 2$ & 11.727000 & 3.237000 & -3.131000 \\
\hline $\mathrm{C} 15$ & 11.923000 & 4.532000 & -2.808000 \\
\hline C16 & 12.298000 & 5.121000 & -3.994000 \\
\hline $\mathrm{N} 4$ & 12.313000 & 4.179000 & -4.972000 \\
\hline N3 & 11.957000 & 3.020000 & -4.445000 \\
\hline $\mathrm{C} 17$ & 12.680000 & 6.537000 & -4.247000 \\
\hline H11 & 12.198000 & 7.176000 & -3.504000 \\
\hline H12 & 12.325000 & 6.845000 & -5.231000 \\
\hline N5 & 14.143000 & 6.727000 & -4.159000 \\
\hline H13 & 14.644000 & 6.881000 & -5.018000 \\
\hline $\mathrm{H} 10$ & 11.833000 & 4.920000 & -1.811000 \\
\hline $\mathrm{H} 29$ & 11.530000 & 1.215000 & -2.756000 \\
\hline $\mathrm{H} 30$ & 11.657000 & 2.226000 & -1.317000 \\
\hline H5 & 5.201000 & 0.344000 & -5.044000 \\
\hline $\mathrm{H} 4$ & 2.758000 & 0.061000 & -4.945000 \\
\hline N6 & 1.304000 & -0.063000 & -2.789000 \\
\hline $\mathrm{H} 17$ & 0.936000 & -0.650000 & -2.043000 \\
\hline $\mathrm{C} 20$ & 0.397000 & 0.979000 & -3.283000 \\
\hline H18 & 0.856000 & 1.513000 & -4.118000 \\
\hline H19 & -0.525000 & 0.518000 & -3.643000 \\
\hline $\mathrm{C} 21$ & 0.073000 & 1.970000 & -2.148000 \\
\hline $\mathrm{H} 20$ & 1.007000 & 2.367000 & -1.755000 \\
\hline $\mathrm{H} 21$ & -0.451000 & 1.450000 & -1.345000 \\
\hline $\mathrm{C} 28$ & -0.792000 & 3.135000 & -2.658000 \\
\hline $\mathrm{N} 7$ & -1.092000 & 4.140000 & -1.656000 \\
\hline $\mathrm{C} 22$ & -2.288000 & 4.674000 & -1.331000 \\
\hline
\end{tabular}




\begin{tabular}{|c|c|c|c|}
\hline $\mathrm{C} 23$ & -1.985000 & 5.642000 & -0.400000 \\
\hline N9 & -0.645000 & 5.647000 & -0.182000 \\
\hline N8 & -0.094000 & 4.723000 & -0.952000 \\
\hline C24 & -2.903000 & 6.611000 & 0.262000 \\
\hline $\mathrm{H} 23$ & -2.421000 & 7.058000 & 1.130000 \\
\hline $\mathrm{H} 24$ & -3.793000 & 6.082000 & 0.611000 \\
\hline N10 & -3.315000 & 7.674000 & -0.676000 \\
\hline $\mathrm{H} 25$ & -2.821000 & 8.550000 & -0.652000 \\
\hline H22 & -3.215000 & 4.385000 & -1.792000 \\
\hline H31 & -0.271000 & 3.633000 & -3.475000 \\
\hline H32 & -1.734000 & 2.747000 & -3.045000 \\
\hline $\mathrm{H} 3$ & 5.459000 & 0.779000 & 1.651000 \\
\hline H1 & 3.025000 & 0.383000 & 1.782000 \\
\hline $\mathrm{C} 2$ & 2.766000 & 0.272000 & -0.354000 \\
\hline $\mathrm{H} 2$ & 1.699000 & 0.086000 & -0.262000 \\
\hline $\mathrm{C} 1$ & 14.861000 & 6.559000 & -3.022000 \\
\hline O1 & 14.293000 & 6.424000 & -1.958000 \\
\hline C3 & 16.385000 & 6.544000 & -3.172000 \\
\hline $\mathrm{C} 4$ & 16.794001 & 5.570000 & -4.319000 \\
\hline H5 & 16.405001 & 5.929000 & -5.271000 \\
\hline H6 & 17.881001 & 5.560000 & -4.408000 \\
\hline N2 & 16.361000 & 4.194000 & -4.140000 \\
\hline $\mathrm{C} 7$ & 16.965000 & 3.226000 & -3.427000 \\
\hline $\mathrm{C} 8$ & 16.187000 & 2.110000 & -3.629000 \\
\hline N4 & 15.147000 & 2.439000 & -4.436000 \\
\hline N3 & 15.259000 & 3.721000 & -4.758000 \\
\hline C11 & 16.475000 & 0.721000 & -3.159000 \\
\hline H14 & 16.666000 & 0.743000 & -2.084000 \\
\hline H15 & 15.606000 & 0.085000 & -3.329000 \\
\hline N8 & 17.658001 & 0.154000 & -3.853000 \\
\hline $\mathrm{H} 18$ & 17.489000 & -0.521000 & -4.582000 \\
\hline $\mathrm{H} 12$ & 17.868000 & 3.370000 & -2.863000 \\
\hline C5 & 17.066999 & 6.079000 & -1.849000 \\
\hline $\mathrm{H7}$ & 18.150000 & 6.033000 & -1.975000 \\
\hline H8 & 16.847000 & 6.758000 & -1.026000 \\
\hline H9 & 16.705999 & 5.096000 & -1.549000 \\
\hline C6 & 16.893999 & 7.968000 & -3.541000 \\
\hline $\mathrm{H} 10$ & 17.931000 & 7.892000 & -3.871000 \\
\hline H11 & 16.319000 & 8.368000 & -4.376000 \\
\hline N5 & 16.848000 & 8.944000 & -2.461000 \\
\hline C9 & 15.813000 & 9.322000 & -1.672000 \\
\hline H13 & 14.822000 & 8.915000 & -1.751000 \\
\hline N6 & 17.983000 & 9.584000 & -2.118000 \\
\hline N7 & 17.684000 & 10.383000 & -1.110000 \\
\hline C10 & 16.371000 & 10.240000 & -0.805000 \\
\hline $\mathrm{C} 12$ & 15.698000 & 11.001000 & 0.296000 \\
\hline H16 & 15.148000 & 11.832000 & -0.148000 \\
\hline H17 & 14.961000 & 10.343000 & 0.762000 \\
\hline N9 & 16.601000 & 11.525000 & 1.354000 \\
\hline H19 & 16.747000 & 12.521000 & 1.380000 \\
\hline $\mathrm{C} 1$ & 18.907000 & 0.674000 & -3.755000 \\
\hline O1 & 19.157000 & 1.496000 & -2.898000 \\
\hline C3 & 19.955999 & 0.250000 & -4.791000 \\
\hline
\end{tabular}




\begin{tabular}{|c|c|c|c|}
\hline $\mathrm{C} 4$ & 19.757999 & 1.087000 & -6.092000 \\
\hline H5 & 19.879999 & 2.145000 & -5.849000 \\
\hline H6 & 20.540001 & 0.832000 & -6.808000 \\
\hline $\mathrm{N} 2$ & 18.479000 & 0.941000 & -6.768000 \\
\hline $\mathrm{C} 7$ & 18.120001 & 0.034000 & -7.706000 \\
\hline $\mathrm{C} 8$ & 16.806000 & 0.345000 & -7.983000 \\
\hline N4 & 16.431000 & 1.408000 & -7.219000 \\
\hline N3 & 17.459999 & 1.779000 & -6.480000 \\
\hline C11 & 15.863000 & -0.330000 & -8.929000 \\
\hline H14 & 16.044001 & 0.040000 & -9.941000 \\
\hline H15 & 16.072001 & -1.403000 & -8.935000 \\
\hline N8 & 14.448000 & -0.111000 & -8.557000 \\
\hline H18 & 13.944000 & 0.569000 & -9.099000 \\
\hline H12 & 18.794001 & -0.709000 & -8.093000 \\
\hline C5 & 19.879000 & -1.273000 & -5.091000 \\
\hline H7 & 20.664000 & -1.558000 & -5.792000 \\
\hline H8 & 20.010000 & -1.847000 & -4.173000 \\
\hline H9 & 18.917999 & -1.546000 & -5.526000 \\
\hline C6 & 21.395000 & 0.585000 & -4.286000 \\
\hline $\mathrm{H} 10$ & 22.117001 & 0.278000 & -5.043000 \\
\hline H11 & 21.492001 & 1.667000 & -4.178000 \\
\hline N5 & 21.796000 & -0.006000 & -3.017000 \\
\hline C9 & 21.570999 & 0.444000 & -1.758000 \\
\hline H13 & 20.955000 & 1.303000 & -1.556000 \\
\hline N6 & 22.568001 & -1.113000 & -3.005000 \\
\hline N7 & 22.822001 & -1.392000 & -1.740000 \\
\hline $\mathrm{C} 10$ & 22.226000 & -0.459000 & -0.951000 \\
\hline C12 & 22.379999 & -0.488000 & 0.535000 \\
\hline $\mathrm{H} 16$ & 22.341000 & 0.530000 & 0.930000 \\
\hline H17 & 21.531000 & -1.028000 & 0.966000 \\
\hline N9 & 23.658001 & -1.128000 & 0.929000 \\
\hline H19 & 24.488001 & -0.673000 & 0.596000 \\
\hline $\mathrm{C} 1$ & 17.224001 & 10.755000 & 2.275000 \\
\hline $\mathrm{O} 1$ & 17.049000 & 9.556000 & 2.281000 \\
\hline C3 & 18.065001 & 11.475000 & 3.339000 \\
\hline $\mathrm{C} 4$ & 19.033001 & 12.520000 & 2.703000 \\
\hline H5 & 18.472000 & 13.263000 & 2.137000 \\
\hline H6 & 19.546000 & 13.055000 & 3.504000 \\
\hline $\mathrm{N} 2$ & 20.051001 & 11.959000 & 1.829000 \\
\hline $\mathrm{C} 7$ & 19.950001 & 11.582000 & 0.535000 \\
\hline $\mathrm{C} 8$ & 21.193001 & 11.077000 & 0.227000 \\
\hline N4 & 21.990999 & 11.198000 & 1.320000 \\
\hline N3 & 21.295000 & 11.735000 & 2.303000 \\
\hline C11 & 21.716000 & 10.510000 & -1.055000 \\
\hline H14 & 21.622999 & 9.421000 & -1.019000 \\
\hline H15 & 21.090000 & 10.851000 & -1.883000 \\
\hline N8 & 23.131001 & 10.900000 & -1.279000 \\
\hline H18 & 23.263000 & 11.874000 & -1.484000 \\
\hline H12 & 19.046000 & 11.680000 & -0.035000 \\
\hline C5 & 18.912001 & 10.450000 & 4.154000 \\
\hline $\mathrm{H} 7$ & 19.520000 & 10.968000 & 4.897000 \\
\hline H8 & 18.275999 & 9.731000 & 4.670000 \\
\hline H9 & 19.572001 & 9.884000 & 3.496000 \\
\hline
\end{tabular}




\begin{tabular}{|c|c|c|c|}
\hline C6 & 17.112000 & 12.231000 & 4.310000 \\
\hline $\mathrm{H} 10$ & 17.711000 & 12.857000 & 4.974000 \\
\hline H11 & 16.458000 & 12.898000 & 3.747000 \\
\hline N5 & 16.271000 & 11.377000 & 5.139000 \\
\hline $\mathrm{C} 9$ & 15.296000 & 10.505000 & 4.780000 \\
\hline H13 & 15.020000 & 10.317000 & 3.758000 \\
\hline N6 & 16.451000 & 11.383000 & 6.477000 \\
\hline N7 & 15.592000 & 10.524000 & 6.987000 \\
\hline C10 & 14.872000 & 9.965000 & 5.978000 \\
\hline C12 & 13.822000 & 8.933000 & 6.256000 \\
\hline H16 & 12.835000 & 9.369000 & 6.090000 \\
\hline H17 & 13.936000 & 8.112000 & 5.542000 \\
\hline N9 & 13.913000 & 8.409000 & 7.642000 \\
\hline H19 & 13.429000 & 8.942000 & 8.342000 \\
\hline $\mathrm{C} 1$ & -4.214000 & 7.491000 & -1.670000 \\
\hline $\mathrm{O} 1$ & -4.851000 & 6.460000 & -1.733000 \\
\hline C3 & -4.351000 & 8.629000 & -2.692000 \\
\hline $\mathrm{C} 4$ & -4.886000 & 9.917000 & -2.002000 \\
\hline $\mathrm{H} 5$ & -4.097000 & 10.353000 & -1.389000 \\
\hline $\mathrm{H} 6$ & -5.152000 & 10.659000 & -2.757000 \\
\hline $\mathrm{N} 2$ & -6.042000 & 9.700000 & -1.148000 \\
\hline C7 & -7.343000 & 9.531000 & -1.483000 \\
\hline $\mathrm{C} 8$ & -7.971000 & 9.292000 & -0.278000 \\
\hline N4 & -7.045000 & 9.343000 & 0.715000 \\
\hline N3 & -5.861000 & 9.590000 & 0.183000 \\
\hline C11 & -9.413000 & 8.999000 & 0.000000 \\
\hline H14 & -9.985000 & 9.925000 & -0.083000 \\
\hline H15 & -9.780000 & 8.302000 & -0.755000 \\
\hline N8 & -9.608000 & 8.405000 & 1.341000 \\
\hline H18 & -8.796000 & 7.942000 & 1.727000 \\
\hline H12 & -7.692000 & 9.581000 & -2.498000 \\
\hline $\mathrm{C} 5$ & -5.340000 & 8.223000 & -3.824000 \\
\hline $\mathrm{H} 7$ & -5.421000 & 9.019000 & -4.565000 \\
\hline $\mathrm{H} 8$ & -5.013000 & 7.313000 & -4.325000 \\
\hline $\mathrm{H} 9$ & -6.333000 & 8.022000 & -3.424000 \\
\hline C6 & -2.962000 & 8.951000 & -3.326000 \\
\hline $\mathrm{H} 10$ & -3.088000 & 9.666000 & -4.140000 \\
\hline H11 & -2.324000 & 9.427000 & -2.581000 \\
\hline N5 & -2.250000 & 7.794000 & -3.846000 \\
\hline C9 & -2.472000 & 7.110000 & -4.991000 \\
\hline H13 & -3.214000 & 7.389000 & -5.715000 \\
\hline N6 & -1.240000 & 7.247000 & -3.136000 \\
\hline N7 & -0.810000 & 6.204000 & -3.826000 \\
\hline $\mathrm{C} 10$ & -1.553000 & 6.087000 & -4.952000 \\
\hline C12 & -1.337000 & 5.001000 & -5.953000 \\
\hline H16 & -1.498000 & 4.035000 & -5.476000 \\
\hline H17 & -2.077000 & 5.099000 & -6.750000 \\
\hline N9 & 0.015000 & 5.046000 & -6.545000 \\
\hline H19 & 0.663000 & 4.326000 & -6.271000 \\
\hline $\mathrm{C} 1$ & -10.692000 & 8.546000 & 2.129000 \\
\hline $\mathrm{O} 1$ & -11.720000 & 9.025000 & 1.675000 \\
\hline $\mathrm{C} 3$ & -10.549000 & 8.027000 & 3.570000 \\
\hline $\mathrm{C} 4$ & -10.622000 & 6.468000 & 3.580000 \\
\hline
\end{tabular}




\begin{tabular}{|c|c|c|c|}
\hline H5 & -9.805000 & 6.057000 & 2.983000 \\
\hline H6 & -10.498000 & 6.099000 & 4.599000 \\
\hline $\mathrm{N} 2$ & -11.866000 & 5.932000 & 3.060000 \\
\hline C7 & -12.993000 & 5.589000 & 3.729000 \\
\hline $\mathrm{C} 8$ & -13.902000 & 5.329000 & 2.725000 \\
\hline N4 & -13.300000 & 5.513000 & 1.524000 \\
\hline N3 & -12.054000 & 5.887000 & 1.725000 \\
\hline C11 & -15.375000 & 5.121000 & 2.783000 \\
\hline H14 & -15.633000 & 4.176000 & 2.299000 \\
\hline H15 & -15.703000 & 5.071000 & 3.825000 \\
\hline N8 & -16.031000 & 6.261000 & 2.096000 \\
\hline H18 & -16.343000 & 6.079000 & 1.157000 \\
\hline H12 & -13.056000 & 5.543000 & 4.800000 \\
\hline C5 & -11.690000 & 8.607000 & 4.456000 \\
\hline $\mathrm{H} 7$ & -11.588000 & 8.257000 & 5.484000 \\
\hline $\mathrm{H} 8$ & -11.662000 & 9.697000 & 4.455000 \\
\hline H9 & -12.670000 & 8.308000 & 4.084000 \\
\hline C6 & -9.177000 & 8.434000 & 4.197000 \\
\hline H10 & -9.119000 & 8.018000 & 5.204000 \\
\hline H11 & -8.356000 & 7.990000 & 3.633000 \\
\hline N5 & -8.923000 & 9.865000 & 4.299000 \\
\hline C9 & -8.381000 & 10.716000 & 3.393000 \\
\hline $\mathrm{H} 13$ & -8.051000 & 10.409000 & 2.418000 \\
\hline N6 & -9.218000 & 10.506000 & 5.449000 \\
\hline N7 & -8.878000 & 11.769000 & 5.288000 \\
\hline C10 & -8.360000 & 11.934000 & 4.042000 \\
\hline C12 & -7.862000 & 13.267000 & 3.567000 \\
\hline H16 & -6.849000 & 13.153000 & 3.175000 \\
\hline $\mathrm{H} 17$ & -8.488000 & 13.603000 & 2.736000 \\
\hline N9 & -7.870000 & 14.292000 & 4.641000 \\
\hline H19 & -7.048000 & 14.341000 & 5.216000 \\
\hline $\mathrm{C} 1$ & 0.505000 & 6.120000 & -7.202000 \\
\hline O1 & -0.231000 & 7.035000 & -7.504000 \\
\hline C3 & 1.991000 & 6.098000 & -7.581000 \\
\hline $\mathrm{C} 4$ & 2.868000 & 5.365000 & -6.513000 \\
\hline H5 & 2.751000 & 4.288000 & -6.642000 \\
\hline H6 & 3.920000 & 5.588000 & -6.695000 \\
\hline $\mathrm{N} 2$ & 2.583000 & 5.675000 & -5.115000 \\
\hline C7 & 2.603000 & 6.865000 & -4.469000 \\
\hline $\mathrm{C} 8$ & 2.222000 & 6.557000 & -3.183000 \\
\hline N4 & 2.010000 & 5.215000 & -3.097000 \\
\hline N3 & 2.227000 & 4.672000 & -4.279000 \\
\hline C11 & 2.058000 & 7.478000 & -2.016000 \\
\hline H14 & 2.849000 & 8.233000 & -2.040000 \\
\hline $\mathrm{H} 15$ & 1.102000 & 7.995000 & -2.114000 \\
\hline N8 & 2.098000 & 6.759000 & -0.726000 \\
\hline H18 & 1.208000 & 6.334000 & -0.475000 \\
\hline H12 & 2.871000 & 7.795000 & -4.933000 \\
\hline C5 & 2.523000 & 7.552000 & -7.744000 \\
\hline $\mathrm{H7}$ & 3.581000 & 7.544000 & -8.008000 \\
\hline H8 & 1.981000 & 8.085000 & -8.526000 \\
\hline H9 & 2.395000 & 8.120000 & -6.825000 \\
\hline C6 & 2.149000 & 5.345000 & -8.935000 \\
\hline
\end{tabular}




\begin{tabular}{|c|c|c|c|}
\hline $\mathrm{H} 10$ & 3.208000 & 5.293000 & -9.191000 \\
\hline H11 & 1.791000 & 4.320000 & -8.828000 \\
\hline N5 & 1.443000 & 5.943000 & -10.061000 \\
\hline C9 & 0.129000 & 5.872000 & -10.391000 \\
\hline $\mathrm{H} 13$ & -0.594000 & 5.332000 & -9.807000 \\
\hline N6 & 2.126000 & 6.703000 & -10.942000 \\
\hline N7 & 1.259000 & 7.128000 & -11.839000 \\
\hline $\mathrm{C} 10$ & 0.026000 & 6.643000 & -11.531000 \\
\hline C12 & -1.167000 & 6.971000 & -12.376000 \\
\hline H16 & -1.504000 & 6.068000 & -12.891000 \\
\hline H17 & -1.986000 & 7.291000 & -11.724000 \\
\hline N9 & -0.872000 & 8.033000 & -13.369000 \\
\hline H19 & -0.425000 & 7.724000 & -14.213000 \\
\hline $\mathrm{C} 1$ & 13.916000 & -0.605000 & -7.421000 \\
\hline $\mathrm{C} 2$ & 12.617000 & 0.009000 & -6.904000 \\
\hline C3 & 12.480000 & 1.512000 & -7.320000 \\
\hline N1 & 13.628000 & 2.431000 & -6.904000 \\
\hline H3 & 14.583000 & 2.076000 & -7.126000 \\
\hline $\mathrm{H} 4$ & 13.643000 & 2.593000 & -5.867000 \\
\hline H16 & 13.543000 & 3.387000 & -7.289000 \\
\hline $\mathrm{H} 1$ & 11.578000 & 1.943000 & -6.868000 \\
\hline $\mathrm{H} 2$ & 12.381000 & 1.602000 & -8.406000 \\
\hline $\mathrm{C} 4$ & 11.453000 & -0.861000 & -7.467000 \\
\hline $\mathrm{N} 2$ & 11.579000 & -2.356000 & -7.158000 \\
\hline $\mathrm{H7}$ & 11.425000 & -2.560000 & -6.154000 \\
\hline H8 & 12.538000 & -2.706000 & -7.372000 \\
\hline H17 & 10.907000 & -2.936000 & -7.694000 \\
\hline H5 & 10.489000 & -0.523000 & -7.070000 \\
\hline H6 & 11.419000 & -0.772000 & -8.559000 \\
\hline C5 & 12.572000 & -0.059000 & -5.350000 \\
\hline H9 & 12.671000 & -1.084000 & -4.988000 \\
\hline $\mathrm{H} 10$ & 13.389000 & 0.512000 & -4.910000 \\
\hline H11 & 11.627000 & 0.339000 & -4.979000 \\
\hline $\mathrm{O} 1$ & 14.409000 & -1.559000 & -6.866000 \\
\hline $\mathrm{C} 1$ & 23.722000 & -2.373000 & 1.438000 \\
\hline $\mathrm{C} 2$ & 25.021999 & -3.169000 & 1.297000 \\
\hline C3 & 25.943001 & -2.643000 & 0.140000 \\
\hline N1 & 25.382000 & -2.739000 & -1.286000 \\
\hline $\mathrm{H} 3$ & 24.474001 & -2.233000 & -1.406000 \\
\hline $\mathrm{H} 4$ & 25.201000 & -3.717000 & -1.577000 \\
\hline H16 & 26.028000 & -2.337000 & -1.992000 \\
\hline H1 & 26.879999 & -3.213000 & 0.137000 \\
\hline $\mathrm{H} 2$ & 26.205999 & -1.594000 & 0.311000 \\
\hline $\mathrm{C} 4$ & 25.736000 & -3.094000 & 2.685000 \\
\hline $\mathrm{N} 2$ & 24.875000 & -3.543000 & 3.872000 \\
\hline $\mathrm{H} 7$ & 24.719000 & -4.567000 & 3.881000 \\
\hline $\mathrm{H} 8$ & 23.922001 & -3.118000 & 3.822000 \\
\hline H17 & 25.285000 & -3.287000 & 4.791000 \\
\hline H5 & 26.645000 & -3.706000 & 2.690000 \\
\hline H6 & 26.025000 & -2.056000 & 2.892000 \\
\hline $\mathrm{C} 5$ & 24.688000 & -4.660000 & 0.987000 \\
\hline H9 & 24.094999 & -5.113000 & 1.784000 \\
\hline $\mathrm{H} 10$ & 24.099001 & -4.745000 & 0.073000 \\
\hline
\end{tabular}




\begin{tabular}{|c|c|c|c|}
\hline H11 & 25.601000 & -5.247000 & 0.870000 \\
\hline O1 & 22.801001 & -2.841000 & 2.063000 \\
\hline $\mathrm{C} 1$ & 24.160999 & 10.177000 & -0.790000 \\
\hline $\mathrm{C} 2$ & 25.482000 & 10.900000 & -0.527000 \\
\hline $\mathrm{C} 3$ & 25.829000 & 11.933000 & -1.650000 \\
\hline N1 & 25.965000 & 11.369000 & -3.067000 \\
\hline $\mathrm{H} 3$ & 25.090000 & 10.928000 & -3.408000 \\
\hline $\mathrm{H} 4$ & 26.707001 & 10.646000 & -3.130000 \\
\hline H16 & 26.216000 & 12.106000 & -3.755000 \\
\hline $\mathrm{H} 1$ & 26.785000 & 12.415000 & -1.414000 \\
\hline $\mathrm{H} 2$ & 25.070000 & 12.722000 & -1.690000 \\
\hline $\mathrm{C} 4$ & 25.351000 & 11.597000 & 0.868000 \\
\hline $\mathrm{N} 2$ & 24.778000 & 10.723000 & 1.989000 \\
\hline $\mathrm{H} 7$ & 25.249001 & 9.804000 & 2.069000 \\
\hline H8 & 23.767000 & 10.515000 & 1.822000 \\
\hline $\mathrm{H} 17$ & 24.820000 & 11.186000 & 2.916000 \\
\hline H5 & 26.330999 & 11.951000 & 1.206000 \\
\hline H6 & 24.681999 & 12.463000 & 0.800000 \\
\hline C5 & 26.655001 & 9.877000 & -0.443000 \\
\hline H9 & 26.504999 & 9.170000 & 0.375000 \\
\hline H10 & 26.732000 & 9.292000 & -1.360000 \\
\hline H11 & 27.605000 & 10.388000 & -0.274000 \\
\hline $\mathrm{O} 1$ & 24.018999 & 9.029000 & -0.447000 \\
\hline $\mathrm{C} 1$ & 14.815000 & 7.473000 & 7.996000 \\
\hline $\mathrm{C} 2$ & 15.246000 & 7.362000 & 9.461000 \\
\hline C3 & 14.963000 & 8.667000 & 10.285000 \\
\hline N1 & 15.725000 & 9.927000 & 9.852000 \\
\hline $\mathrm{H} 3$ & 15.584000 & 10.162000 & 8.843000 \\
\hline $\mathrm{H} 4$ & 16.749001 & 9.833000 & 9.977000 \\
\hline H16 & 15.441000 & 10.768000 & 10.390000 \\
\hline H1 & 15.225000 & 8.498000 & 11.336000 \\
\hline $\mathrm{H} 2$ & 13.895000 & 8.911000 & 10.255000 \\
\hline $\mathrm{C} 4$ & 14.487000 & 6.128000 & 10.047000 \\
\hline N2 & 14.669000 & 4.835000 & 9.244000 \\
\hline $\mathrm{H} 7$ & 15.607000 & 4.414000 & 9.379000 \\
\hline $\mathrm{H} 8$ & 14.595000 & 5.026000 & 8.219000 \\
\hline H17 & 13.968000 & 4.107000 & 9.476000 \\
\hline H5 & 14.802000 & 5.923000 & 11.076000 \\
\hline H6 & 13.409000 & 6.332000 & 10.057000 \\
\hline C5 & 16.778999 & 7.086000 & 9.531000 \\
\hline H9 & 17.042000 & 6.154000 & 9.027000 \\
\hline $\mathrm{H} 10$ & 17.340000 & 7.878000 & 9.033000 \\
\hline H11 & 17.115000 & 7.015000 & 10.568000 \\
\hline O1 & 15.234000 & 6.679000 & 7.189000 \\
\hline $\mathrm{C} 1$ & -15.865000 & 7.529000 & 2.527000 \\
\hline $\mathrm{C} 2$ & -16.054001 & 8.668000 & 1.526000 \\
\hline C3 & -15.398000 & 8.334000 & 0.143000 \\
\hline N1 & -13.898000 & 8.016000 & 0.159000 \\
\hline H3 & -13.677000 & 7.132000 & 0.669000 \\
\hline $\mathrm{H} 4$ & -13.315000 & 8.741000 & 0.629000 \\
\hline H16 & -13.509000 & 7.890000 & -0.792000 \\
\hline $\mathrm{H} 1$ & -15.524000 & 9.186000 & -0.534000 \\
\hline $\mathrm{H} 2$ & -15.892000 & 7.473000 & -0.320000 \\
\hline
\end{tabular}




\begin{tabular}{|c|c|c|c|}
\hline $\mathrm{C} 4$ & -17.587999 & 8.922000 & 1.405000 \\
\hline $\mathrm{N} 2$ & -18.296000 & 9.202000 & 2.735000 \\
\hline H7 & -18.013000 & 10.112000 & 3.143000 \\
\hline H8 & -18.069000 & 8.484000 & 3.454000 \\
\hline H17 & -19.329000 & 9.227000 & 2.639000 \\
\hline H5 & -17.788000 & 9.773000 & 0.743000 \\
\hline H6 & -18.076000 & 8.040000 & 0.975000 \\
\hline C5 & -15.373000 & 9.971000 & 2.047000 \\
\hline H9 & -15.803000 & 10.296000 & 2.996000 \\
\hline H10 & -14.308000 & 9.810000 & 2.221000 \\
\hline H11 & -15.487000 & 10.783000 & 1.326000 \\
\hline $\mathrm{O} 1$ & -15.619000 & 7.764000 & 3.685000 \\
\hline $\mathrm{C} 1$ & -8.991000 & 14.950000 & 5.001000 \\
\hline $\mathrm{C} 2$ & -9.050000 & 15.596000 & 6.388000 \\
\hline C3 & -8.160000 & 14.842000 & 7.437000 \\
\hline N1 & -8.532000 & 13.383000 & 7.730000 \\
\hline $\mathrm{H} 3$ & -8.548000 & 12.788000 & 6.871000 \\
\hline H4 & -9.473000 & 13.291000 & 8.155000 \\
\hline H16 & -7.868000 & 12.931000 & 8.388000 \\
\hline $\mathrm{H} 1$ & -8.205000 & 15.368000 & 8.398000 \\
\hline $\mathrm{H} 2$ & -7.113000 & 14.844000 & 7.118000 \\
\hline $\mathrm{C} 4$ & -8.608000 & 17.084000 & 6.214000 \\
\hline $\mathrm{N} 2$ & -9.388000 & 17.865999 & 5.151000 \\
\hline $\mathrm{H} 7$ & -10.385000 & 17.985001 & 5.406000 \\
\hline $\mathrm{H} 8$ & -9.391000 & 17.368999 & 4.236000 \\
\hline H17 & -9.000000 & 18.815001 & 4.987000 \\
\hline H5 & -8.714000 & 17.629000 & 7.160000 \\
\hline H6 & -7.554000 & 17.127001 & 5.918000 \\
\hline $\mathrm{C} 5$ & -10.515000 & 15.572000 & 6.921000 \\
\hline H9 & -11.188000 & 16.129000 & 6.268000 \\
\hline $\mathrm{H} 10$ & -10.895000 & 14.550000 & 6.969000 \\
\hline H11 & -10.572000 & 16.011999 & 7.919000 \\
\hline O1 & -9.909000 & 15.087000 & 4.228000 \\
\hline $\mathrm{C} 1$ & 3.232000 & 6.224000 & -0.239000 \\
\hline $\mathrm{C} 2$ & 3.110000 & 5.138000 & 0.829000 \\
\hline $\mathrm{C} 3$ & 1.965000 & 5.426000 & 1.851000 \\
\hline N1 & 1.988000 & 6.804000 & 2.510000 \\
\hline $\mathrm{H} 3$ & 1.947000 & 7.569000 & 1.810000 \\
\hline $\mathrm{H} 4$ & 2.844000 & 6.954000 & 3.076000 \\
\hline H16 & 1.181000 & 6.935000 & 3.150000 \\
\hline H1 & 1.997000 & 4.684000 & 2.657000 \\
\hline $\mathrm{H} 2$ & 0.994000 & 5.326000 & 1.359000 \\
\hline $\mathrm{C} 4$ & 2.867000 & 3.782000 & 0.101000 \\
\hline $\mathrm{N} 2$ & 3.611000 & 3.618000 & -1.221000 \\
\hline H7 & 4.592000 & 3.947000 & -1.155000 \\
\hline H8 & 3.169000 & 4.195000 & -1.972000 \\
\hline $\mathrm{H} 17$ & 3.617000 & 2.639000 & -1.559000 \\
\hline H5 & 3.172000 & 2.955000 & 0.751000 \\
\hline H6 & 1.805000 & 3.656000 & -0.129000 \\
\hline C5 & 4.442000 & 5.018000 & 1.624000 \\
\hline H9 & 5.267000 & 4.732000 & 0.969000 \\
\hline $\mathrm{H} 10$ & 4.707000 & 5.971000 & 2.085000 \\
\hline H11 & 4.360000 & 4.263000 & 2.408000 \\
\hline
\end{tabular}




\begin{tabular}{|c|c|c|c|}
\hline O1 & 4.307000 & 6.497000 & -0.715000 \\
\hline C1 & -0.919000 & 9.344000 & -13.060000 \\
\hline C2 & -0.118000 & 10.340000 & -13.903000 \\
\hline C3 & 1.071000 & 9.672000 & -14.680000 \\
\hline N1 & 2.190000 & 9.059000 & -13.828000 \\
\hline H3 & 1.842000 & 8.333000 & -13.162000 \\
\hline H 16 & 2.679000 & 9.762000 & -13.244000 \\
\hline H1 & 2.920000 & 8.595000 & -14.403000 \\
\hline H2 & 1.552000 & 10.421000 & -15.321000 \\
\hline C4 & -1.698000 & 8.879000 & -15.337000 \\
\hline N2 & -2.359000 & 11.010000 & -14.877000 \\
\hline H7 & -2.124000 & 12.535000 & -14.190000 \\
\hline H8 & -2.748000 & 10.990000 & -13.685000 \\
\hline H17 & -3.125000 & 11.860000 & -14.465000 \\
\hline H5 & -0.659000 & 11.798000 & -15.4690000 \\
\hline H6 & -1.532000 & 10.258000 & -15.571000 \\
\hline C5 & 0.486000 & 11.439000 & -12.977000 \\
\hline H9 & -0.293000 & 11.990000 & -12.447000 \\
\hline H10 & 1.128000 & 10.995000 & -12.213000 \\
\hline H11 & 1.073000 & 12.155000 & -13.554000 \\
\hline O1 & -1.639000 & 9.750000 & -12.178000 \\
\hline
\end{tabular}

\section{References}

(1) Ma, Y.; Tang, Q.-Y.; Zhu, J.; Wang, L.-H.; Yao, C. Fluorescent and Thermal Properties of Siloxane-Polyurethanes Based on 1,8-Naphthalimide. Chinese Chem. Lett. 2014, 25 (5), 680-686. https://doi.org/10.1016/J.CCLET.2014.01.048.

(2) Marion, A.; Góra, J.; Kracker, O.; Fröhr, T.; Latajka, R.; Sewald, N.; Antes, I. Amber-Compatible Parametrization Procedure for Peptide-like Compounds: Application to 1,4- and 1,5-Substituted Triazole-Based Peptidomimetics. J. Chem. Inf. Model. 2018, 58 (1), 90-110. https://doi.org/10.1021/acs.jcim.7b00305.

(3) Wang, J.; Wolf, R. M.; Caldwell, J. W.; Kollman, P. A.; Case, D. A. Development and Testing of a General Amber Force Field. J. Comput. Chem. 2004, 25 (9), $1157-$ 1174. https://doi.org/10.1002/jcc.20035.

(4) M. J. Frisch, G. W. Trucks, H. B. Schlegel, G. E. Scuseria, M. A. Robb, J. R. Cheeseman, G. Scalmani, V. Barone, G. A. Petersson, H. Nakatsuji, X. Li, M. Caricato, A. Marenich, J. Bloino, B. G. Janesko, R. Gomperts, B. Mennucci, H. P. Hratchian, J. V. Ort, and D. J. F. Gaussian 09, Revision A.02. Gaussian, Inc., Wallingford CT 2016.

(5) Tomasi, J.; Mennucci, B.; Cammi, R. Quantum Mechanical Continuum Solvation Models. Chem. Rev. 2005, 105 (8), 2999-3094. https://doi.org/10.1021/cr9904009.

(6) Bayly, C. I.; Cieplak, P.; Cornell, W.; Kollman, P. A. A Well-Behaved 
Electrostatic Potential Based Method Using Charge Restraints for Deriving Atomic Charges: The RESP Model. J. Phys. Chem. 1993, 97 (40), 10269-10280. https://doi.org/10.1021/j100142a004.

(7) D. A. Case, T. A. Darden, T. E. Cheatham, III, C. L. Simmerling, J. Wang, R. E. Duke, R. Luo, R. C. Walker, W. Zhang, K. M. Merz, B. P. Roberts, S. Hayik, A. E. Roitberg, G. Seabra, J. M. Swails, I. Kolossváry, K. F. Wong, F. Paesani, J. Vanicek, R. M. Wo, A. K. and P. A. K. AMBER 12, University of California, San Francisco. 2012.

(8) Maingi, V.; Jain, V.; Bharatam, P. V.; Maiti, P. K. Dendrimer Building Toolkit: Model Building and Characterization of Various Dendrimer Architectures. $J$. Comput. Chem. 2012, 33 (25), 1997-2011. https://doi.org/10.1002/jcc.23031.

(9) Jorgensen, W. L.; Chandrasekhar, J.; Madura, J. D.; Impey, R. W.; Klein, M. L. Comparison of Simple Potential Functions for Simulating Liquid Water. J. Chem. Phys. 1983, 79 (2), 926-935. https://doi.org/10.1063/1.445869.

(10) Ruiz-Sanchez, A. J.; Mesa-Antunez, P.; Barbero, N.; Collado, D.; Vida, Y.; Najera, F.; Perez-Inestrosa, E. Synthesis of All-Aliphatic Polyamide Dendrimers Based on a 3,3'-Diaminopivalic Acid Scaffold. Polym. Chem. 2015, 6 (16), 3031-3038. https://doi.org/10.1039/C5PY00154D.

(11) Verlet, L. Computer "Experiments" on Classical Fluids. I. Thermodynamical Properties of Lennard-Jones Molecules. Phys. Rev. 1967, 159 (1), 98-103. https://doi.org/10.1103/PhysRev.159.98.

(12) Ryckaert, J.-P.; Ciccotti, G.; Berendsen, H. J. . Numerical Integration of the Cartesian Equations of Motion of a System with Constraints: Molecular Dynamics of n-Alkanes. J. Comput. Phys. 1977, 23 (3), 327-341. https://doi.org/10.1016/0021-9991(77)90098-5.

(13) Berendsen, H. J. C.; Postma, J. P. M.; van Gunsteren, W. F.; DiNola, A.; Haak, J. R. Molecular Dynamics with Coupling to an External Bath. J. Chem. Phys. 1984, 81 (8), 3684-3690. https://doi.org/10.1063/1.448118.

(14) Darden, T.; York, D.; Pedersen, L. Particle Mesh Ewald: An $N \cdot \log (N)$ Method for Ewald Sums in Large Systems. J. Chem. Phys. 1993, 98 (12), 10089-10092. https://doi.org/10.1063/1.464397.

(15) Humphrey, W.; Dalke, A.; Schulten, K. VMD: Visual Molecular Dynamics. J. Mol. Graph. 1996, 14 (1), 33-38. https://doi.org/10.1016/0263-7855(96)00018-5.

(16) Rudnick, J.; Gaspari, G. The Aspherity of Random Walks. J. Phys. A. Math. Gen. 1986, 19 (4), L191-L193. https://doi.org/10.1088/0305-4470/19/4/004. 